\title{
Global Compact Passive Macromodeling Algorithms for High-Speed Circuits
}

\author{
by \\ Dharmendra Saraswat, B. E., M.A.Sc, \\ A thesis submitted to the Faculty of Graduate Studies and Research \\ in partial fulfillment of the requirements for the degree of \\ Doctor of Philosophy
Ottawa-Carleton Institute for Electrical Engineering
Department of Electronics
Faculty of Engineering
Carleton University
Ottawa, Ontario, Canada

(C) Dharmendra Saraswat, 2006 


$\begin{array}{ll}\begin{array}{l}\text { Library and } \\ \text { Archives Canada }\end{array} & \begin{array}{l}\text { Bibliothèque et } \\ \text { Archives Canada }\end{array} \\ \begin{array}{l}\text { Published Heritage } \\ \text { Branch }\end{array} & \begin{array}{l}\text { Direction du } \\ \text { Patrimoine de l'édition }\end{array} \\ \begin{array}{l}\text { 395 Wellington Street } \\ \text { Ottawa ON K1A 0N4 }\end{array} & \begin{array}{l}\text { 395, rue Wellington } \\ \text { Ottana ON K1A ON4 } \\ \text { Canada Oa }\end{array}\end{array}$

Your file Votre référence ISBN: 978-0-494-18236-9 Ourfile Notre référence ISBN: $978-0-494-18236-9$

NOTICE:

The author has granted a nonexclusive license allowing Library and Archives Canada to reproduce, publish, archive, preserve, conserve, communicate to the public by telecommunication or on the Internet, loan, distribute and sell theses worldwide, for commercial or noncommercial purposes, in microform, paper, electronic and/or any other formats.

The author retains copyright ownership and moral rights in this thesis. Neither the thesis nor substantial extracts from it may be printed or otherwise reproduced without the author's permission.
AVIS:

L'auteur a accordé une licence non exclusive permettant à la Bibliothèque et Archives Canada de reproduire, publier, archiver, sauvegarder, conserver, transmettre au public par télécommunication ou par l'Internet, prêter, distribuer et vendre des thèses partout dans le monde, à des fins commerciales ou autres, sur support microforme, papier, électronique et/ou autres formats.

L'auteur conserve la propriété du droit d'auteur et des droits moraux qui protège cette thèse. $\mathrm{Ni}$ la thèse ni des extraits substantiels de celle-ci ne doivent être imprimés ou autrement reproduits sans son autorisation.
In compliance with the Canadian

Privacy Act some supporting forms may have been removed from this thesis.

While these forms may be included in the document page count, their removal does not represent any loss of content from the thesis.
Conformément à la loi canadienne sur la protection de la vie privée, quelques formulaires secondaires ont été enlevés de cette thèse.

Bien que ces formulaires aient inclus dans la pagination, il n'y aura aucun contenu manquant. 


\begin{abstract}
In the recent years, signal integrity has been at the forefront of new developments in $C A D$ algorithms focussed on high-speed electronic circuits. With the continuous rise in operating frequencies, the interconnect effects such as delay, distortion, crosstalk and ringing become the dominant factors limiting the performance of VLSI systems. At relatively higher frequencies and increased level of integration, it is not always possible to find analytical or simulatable models for high-speed modules such as packages, vias, connectors, nonuniform transmission lines etc. In such a scenario, these devices are characterized by tabulated multiport parameters ( $S, Y, Z$ or $T$ ), obtained either through measurements or from the physical layout using rigorous full-wave electromagnetic simulations. However, transient simulation of such frequency-dependent tabulated data in the presence of nonlinear devices to obtain a global electrical assessment is a CPU expensive process due to the mixed frequency/time problem. Recently, several macromodeling algorithms have been proposed for this purpose. However, a major difficulty associated with these algorithms is that, they are either not able to guarantee the passivity of the resulting macromodel or when guaranteeing the passivity, they become prohibitively CPU expensive. In addition, further challenges are posed by modern designs which tend to contain multiple diverse high-speed modules. Such an emerging scenario typically leads to large sized circuit matrices and warrants unified passive reduction algorithms to facilitate fast simulations. However, the currently available algorithms cannot perform global passive reduction of such an environment with multiple diverse high-speed modules.

In this thesis, novel algorithms are presented to address the above issues. A systematic methodology is presented for the passive macromodeling of tabulated data networks. For this purpose, fast passivity verification and enforcement algorithms are presented. These algorithms are suitable for small as well as large sized macromodels of tabulated networks. In addition, an algorithm with a guaranteed search direction is presented, to reduce the number of iterations during the passivity enforcement process. Also, a new algorithm is presented for a global reduction of circuits containing diverse high-speed modules. This algorithm permits a novel formulation to guarantee the passivity of the resulting reducedorder model. Also, an efficient algorithm is presented for the second-level reduction, to obtain further compact macromodels. The developed algorithms are robust and practical, and suitable for implementation in state-of-the-art design automation tools.
\end{abstract}




\section{Acknowledgments}

I would like to express my gratitude and appreciation to Professor Ram Achar and Professor Michel Nakhla for their expert guidance, helpful suggestions and much needed encouragement. Their ever friendly manner, motivation in difficult moments, patience and good judgement made the journey through this thesis an enjoyable and unforgettable experience. I feel privileged to have them as my thesis supervisors.

I would like to acknowledge and thank several of my colleagues: A. Charest, C. Chen, Dr. A. Dounavis, L. Filipovic, Dr. E. Gad, Dr. Pavan Gunupudi, K. Harb, A. Jerome, Dr. R. Khazaka, G. Lo, N. Nakhla, B. S. Nouri, P. Pai, D. Paul, G. S. Shinh, Dr. Nick Soveiko, Y. Xu and Y. Zhou, for their cooperation and making my stay here an enjoyable experience. Special thanks are due to Andrew and Lidija for proof reading my thesis. The office staff of Betty Zahalan, Peggy Poccolo, Lorena Duncan, Jacques Lemieux, Scott Bruce were more than helpful in the hour of need. I would also like to express my gratitude to the professors and students at the department of electronics for making my work a pleasant experience. Financial support from MICRONET, NSERC, Ministry of Training, Colleges and Universities of Ontario, is greatly acknowledged.

I leave a special note of appreciation for my wife, Preeti (Dolly). Without her loving support, encouragement and patience, working on this thesis would have been hard. My kids, Shreya and Anish have a special place in my heart. Their smile and love provided the much needed relaxation after a hard day's work. I extend thanks to my father and mother-in-law, brother-in-law Sanjay and Deepak, sister-in-law Kanika for their support and making my stay at Ottawa pleasant and comfortable. Friends, relatives, cousins, uncles, aunts and in particular grand parents are remembered for being with me all the time. My final thoughts are with my parents, my sister late Smt. Pushplata, brother-in-law Shri Mohan Swaroop Saraswat, my brother Jitendra, sister-in-law Vineeta and niece Shipi, for patient backing in everything, I am grateful to you. I believe your patience finally paid off. 
Dedicated to my parents

\section{Shri Radhey Shyam Saraswat}

\section{Smt. Mithlesh Saraswat}

for love, affection and sacrifice for their kids 


\section{Table of Contents}

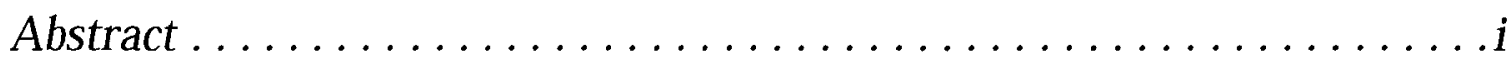

Acknowledgments.........................

Glossary of Terms .................................

CHAPTER 1 Introduction..$\ldots \ldots \ldots \ldots \ldots \ldots \ldots \ldots \ldots \ldots \ldots \ldots \ldots \ldots$

$1.1 \quad$ Background and Motivation $\ldots \ldots \ldots \ldots \ldots \ldots \ldots \ldots \ldots$

1.2 Contributions............................ 5

1.3 Organization of the Thesis. ..................... 7

CHAPTER 2 Review of Modeling/Simulation of Tabulated Data Subnetworks .. 8

2.1 Modeling/Simulation of Subnetworks Characterized by

Tabulated Data $\ldots \ldots \ldots \ldots \ldots \ldots \ldots \ldots \ldots$

2.2 Convolution Based Transient Analysis ............... 10

2.3 Rational Function Based Approaches .................. 14

2.3.1 Standard Least-Square . . . . . . . . . . . . . . . . . 15

2.3.1a Computation of Residues.................... 18

2.3.2 Vector Fitting by Pole Relocation $\ldots \ldots \ldots \ldots \ldots \ldots 21$

2.3.2a Pole Identification Algorithm ..................22

2.3.2b Refinement of Poles of $f(s) \ldots \ldots \ldots \ldots \ldots \ldots 27$

2.3.2c Evaluation of Zeros of $\sigma(s)$ (i.e. poles of $f(s)) \ldots \ldots .29$

2.3.2d Selection of Initial Set of Poles................ 30

2.3.2e Residue Calculation ........................ 31

2.3.2f Calculation of Common Pole-Set for Multiport

Networks ............................. 31

2.3.3 Rational Function Approximation via Nonlinear

Formulation ........................... 32 


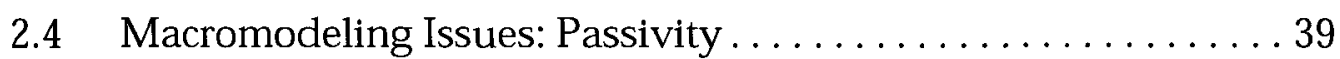

2.5 Review of Passive Macromodeling Algorithms for Tabulated Data....................................... 40

2.5.1 Passive Rational Function Approximation Using Nevanlinna-Pick Interpolation (NPI) . . . . . . . . . 40

2.5.2 Convex Programming Approach to Positive-Real Rational Approximation ................... 44

2.5.2a Formulation of the Optimization Problem .......... 45

2.6 Conclusion................................. 49

CHAPTER $3 \quad$ Review of Model Order Reduction Techniques ............50

3.1 Direct Moment Matching Techniques ................ 51

3.2 Indirect Moment Matching Technique ............... 52

3.3 Truncated Balanced Realization ................... 55

3.3.1 Standard TBR .......................... 56

3.3.2 PR-TBR based Second Level Reduction............ 58

3.4 Conclusion ............................... 59

\section{CHAPTER 4 Passive Macromodeling of Subnetworks Characterized by Tabulated}

Data.......................................61

4.1 Problem Formulation . . . . . . . . . . . . . . . . . . 62

4.2 Proposed Passive Macromodeling Algorithm ............ 64

4.2.1 First Step: Approximation . . . . . . . . . . . . . . . 66

4.2.2 Second Step: Passivity Check $\ldots \ldots \ldots \ldots \ldots \ldots \ldots 67$

4.2.3 Third Step: Determination of Passivity Violating Regions [47], [50] - [53]. . . . . . . . . . . . 74

4.2.3a Determination of Location of Maximum Violation in a Non-Passive Region .................... 77

4.2.4 Fourth Step: Passivity Enforcement . . . . . . . . . . . 78 
4.2.4a Compensation by First-Order Perturbation (PCFOP) . . 78

4.2.4b Compensation by Linear Matrix Inequality (PCLMI) . . 84

4.3 Numerical Results . . . . . . . . . . . . . . . . . . . . . . . . . . . 90

4.3.1 Example 1: Three Port Transmission Line Network. . . . 90

4.3.2 Example 2: Coaxial to Microstrip Coupler . . . . . . . . . 94

4.3.3 Example 3: Two Coupled Line. . . . . . . . . . . . . . 97

4.3.4 Example 4: RJ-45 Connector. . . . . . . . . . . . . 100

4.3.5 Example 5: Multilayer Oval Via ............. 103

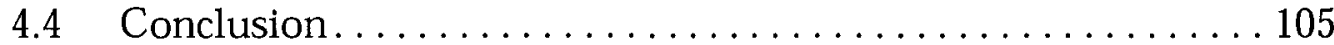

CHAPTER 5 Passive Macromodeling of Tabulated Networks with Large-Order $\begin{array}{ll}\text { Macromodels } & 106\end{array}$

5.1 Problem Formulation and Preliminaries. . . . . . . . . 108

5.2 Proposed Algorithm for Passivity Verification/ Enforcement of Large- Order Macromodels. . . . . . . . . . . . . . . . . 110

5.2.1 Quantification of Passivity Violation of Large Scale Systems....................... 110

5.2.1a Frequency-Sweep of the Region Between $f_{\max }$ and $\infty$ via Reciprocal Systems . . . . . . . . . 111

5.2.1b Computational Considerations of Constructing the Reciprocal System . . . . . . . . . . . . . . . . . 113

5.2.1c Computational Considerations of Frequency Sweep. . . 114

5.2.1d Adaptive Approach for Determining the Magnitude of the Maximum Violation. . . . . . . . . . . 117

5.3 Proposed Passivity Enforcement . . . . . . . . . . . 118

5.3.1a Fast Solution of Lyapunov Equation . . . . . . . . . . 120

5.3.1b Discussion of Computational Complexity. . . . . . . . . 121

5.4 Guidelines for Passivity Verification During the Intermediate Steps of the Compensation Process for Large Scale Systems . . 122 
$5.5 \quad$ Numerical Results . . . . . . . . . . . . . . . . . . . . . 125

5.5.1 Example 1: IC Package .................... 126

5.5.2 Example 2: 12-Port Network . . . . . . . . . . . . . 130

5.6 Conclusion................................ 135

CHAPTER 6 Global Passivity Enforcement Algorithm for Macromodels of Tabulated

Data...................................... 136

6.1 Problem Formulation. ........................ 137

6.2 Passivity Verification $\ldots \ldots \ldots \ldots \ldots \ldots \ldots \ldots \ldots \ldots \ldots \ldots$

6.3 Passivity Enforcement ........................ 140

6.3.1 Determination of Passivity Violation Regions ....... 141

6.3.2 Passivity Enforcement with Guaranteed Search

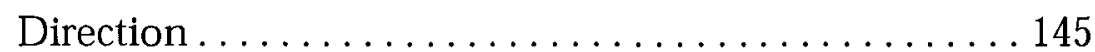

6.3.2a Selection of Appropriate Residues for Perturbation ... 146

6.3.2b Error Estimation........................ 150

$6.4 \quad$ Numerical Results . . . . . . . . . . . . . . . . . . . . . . 152

6.4.1 Example 1: Three Port Interconnect Network........ 152

6.4.2 Example 2: Four Port Tabulated Data............ 162

6.5 Conclusion................................. 169

CHAPTER 7 Global Passive Reduction Algorithm for RLC Interconnect

Circuits with Embedded State-Space Systems . . . . . . . 170

7.1 Limitation of PRIMA Type of First-level Algorithms . . . . . . 172

7.2 Proposed Reduction of RLC Interconnects with Embedded

State-Space Systems (PRESS) . . . . . . . . . . . . . . . . 176

7.2.1 Formulation of Unified Network Equations ......... 176

7.2.2 Passive Model-Order Reduction ............... 178

7.2.3 Proof of Passivity Preservation ................. 179 
7.2.4 Computation of Matrix $\boldsymbol{P} \ldots \ldots \ldots \ldots \ldots \ldots \ldots$

$7.3 \quad$ Numerical Results . . . . . . . . . . . . . . . . . . . . . . 185

7.3.1 Example 1: Two Port RLC Network with Embedded Subnetwork ............................... 185

7.3.2 Example 2: Two Port RLC Network with Embedded Subnetwork ........................... 187

7.3.3 Example 3: Three Port RLC Network with Embedded State-Space System . . . . . . . . . . . . . . . . . . . . 192

7.4 Development of the Proposed Methodology for Generating Compact Macromodels ............................ 198

7.4.1 Proposed Fast Passive Truncated Balanced Realization (FPTBR) . . . . . . . . . . . . . . . . . . . . . . . . . . . . . 198

7.5 Numerical Results . . . . . . . . . . . . . . . . . . . . . . . 202

7.5.1 Example: Four Port Network.................. 202

7.6 Performance Comparison of the Proposed two-level GMOR Technique................................. 204

7.6.1 Example 1: Two Port Network ................ 205

7.6.2 Example 2: Four Port Network ................ 208

7.7 Conclusion..................................... 212

CHAPTER 8 Conclusions and Future Work $\ldots \ldots \ldots \ldots \ldots \ldots \ldots \ldots \ldots \ldots \ldots \ldots \ldots \ldots \ldots$

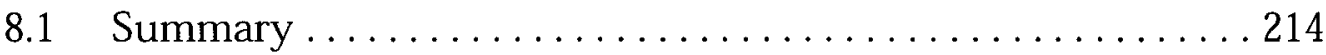

$8.2 \quad$ Future Research.............................. 217

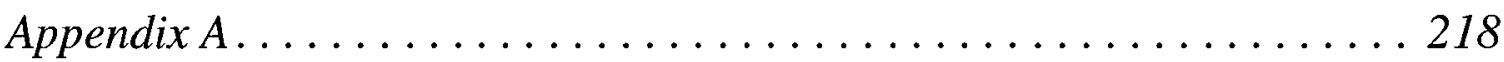

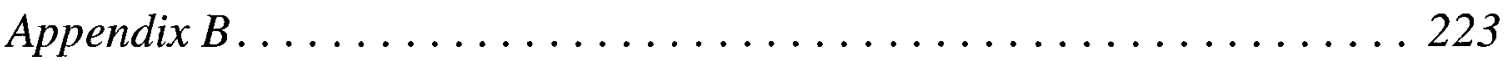

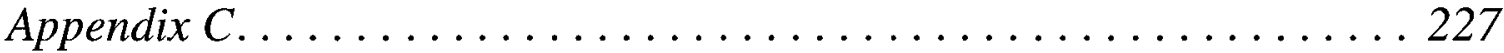

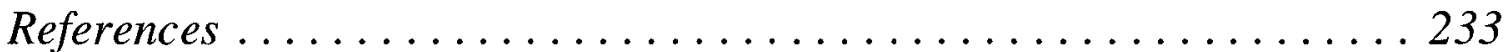




\section{List of Figures}

Fig. 1.1. High-speed interconnect effects $\ldots \ldots \ldots \ldots \ldots \ldots \ldots \ldots \ldots$

Fig. 1.2. Linear subnetwork with diverse high-speed modules (distributed, EM,

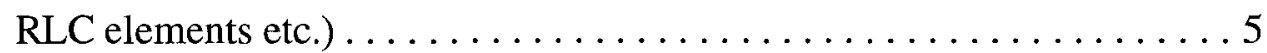

Fig. 2.1. Commonly adopted modeling/simulation flow for tabulated networks . 10

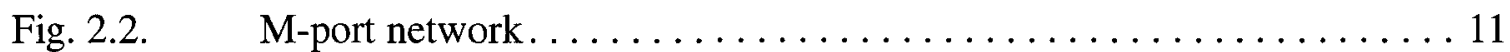

Fig. 2.3. Transient response of a stable but non-passive macromodel with passive termination . . . . . . . . . . . . . . . . . 40

Fig. 4.1. Illustration of significance of passivity. $\ldots \ldots \ldots \ldots \ldots \ldots \ldots$

Fig. 4.2. Flow diagram of the proposed passive macromodeling algorithm. . . 65

Fig. 4.3. Illustration of identification of regions of passivity violation $\ldots \ldots 76$

Fig. 4.4. Passivity compensation by perturbation of matrix $C \ldots \ldots \ldots \ldots$

Fig. 4.5. Pseudocode of the proposed passive macromodeling algorithm . . . 88

Fig. 4.6. $\quad$ Eigenvalue spectrum of Hamiltonian matrix $\ldots \ldots \ldots \ldots \ldots \ldots$

Fig. 4.7. Comparison of norm of $\boldsymbol{S}(j \omega)$ : Three port network ........92

Fig. 4.8. S-parameter responses: Three port network $\quad \ldots \ldots \ldots \ldots \ldots \ldots \ldots$

Fig. 4.9. Transient response: Three port network . . . . . . . . . . . 94

Fig. 4.10. Comparison of norm of $S(j \omega)$ : Coaxial to microstrip coupler.....95

Fig. 4.11. S-parameter responses: Coaxial to microstrip coupler . . . . . . 96

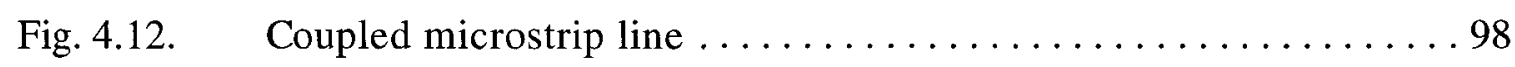

Fig. 4.13. Comparison of norm of $\boldsymbol{S}(j \omega)$ : Coupled microstrip line . . . . . . 98

Fig. 4.14. S-parameter responses: Coupled microstrip line . . . . . . . . . 99

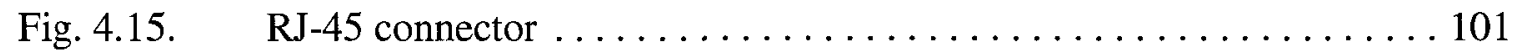

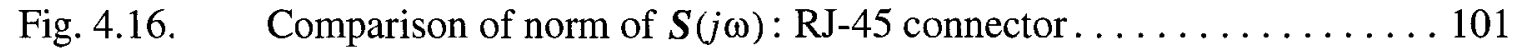

Fig. 4.17. S-parameter responses: RJ-45 connector $\ldots \ldots \ldots \ldots \ldots \ldots \ldots \ldots$

Fig. 4.18. Comparison of norm of $\boldsymbol{S}(j \omega)$ : Multilayer oval via. . . . . . . 103

Fig. 4.19. S-parameter responses: Multilayer oval via . . . . . . . . . . . . 104

Fig. 5.1. Mapping from original domain to reciprocal domain. . . . . . . 112 
Fig. 5.2. Determination of locations of maximum passivity violation

Fig. 5.3. Norm-spectrum with multiple regions of violation and compensation by iterative perturbation of matrix $C$.

Fig. 5.4. Pseudocode for the proposed passive macromodeling algorithm

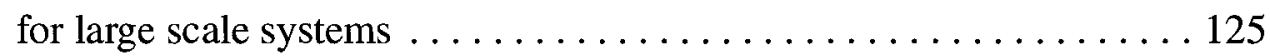

Fig. 5.5. Comparison of norm of $S(j \omega)$ : IC package $\ldots \ldots \ldots \ldots \ldots \ldots$

Fig. 5.6. Identification of passivity violation beyond $f_{\max } \ldots \ldots \ldots \ldots \ldots$

Fig. 5.7. Comparison of sample S-parameters: IC package. . . . . . . . . . . 129

Fig. 5.8. Comparison of norm of $S(j \omega)$ : example $2 \ldots \ldots \ldots \ldots \ldots \ldots$

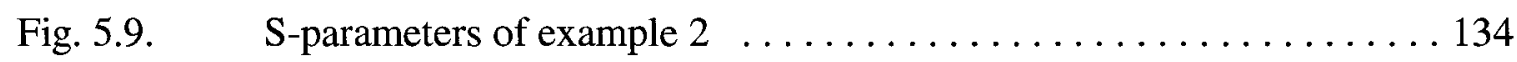

Fig. 6.1. Illustration of identification of regions of passivity violation . . . . 144

Fig. 6.2. Pseudocode for the proposed passivity compensation algorithm . . . . 151

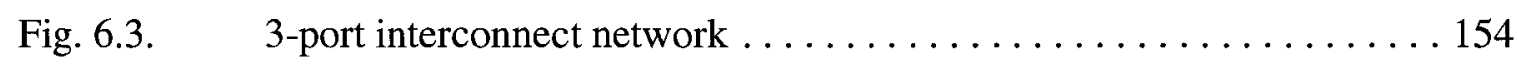

Fig. 6.4. (a) Eigenvalue spectrum of Hamiltonian matrix

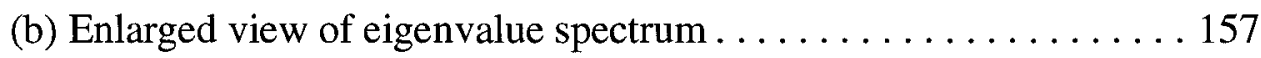

Fig. 6.5. Eigenvalue v/s frequency of $\operatorname{Real}(Y(j \omega))$ - example $1 \ldots \ldots \ldots 158$

Fig. 6.6. $\quad$ Eigenvalue v/s frequency of $\operatorname{Real}(Y(j \omega))$ - with proposed passivity compensation ....................... 158

Fig. 6.7. Frequency responses (magnitude) - example $1 \ldots \ldots \ldots \ldots \ldots$. . . . . 159

Fig. 6.8. Frequency responses (real part) - example $1 \ldots \ldots \ldots \ldots \ldots \ldots$

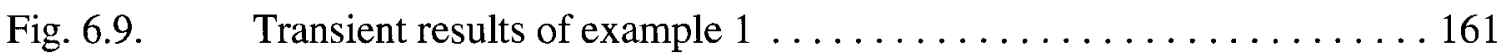

Fig. 6.10. Four port interconnect subnetwork - example $2 \ldots \ldots \ldots \ldots \ldots$

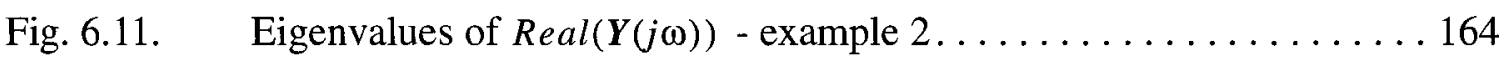

Fig. 6.12. Sample admittance parameters (real part) - example 2........ 166

Fig. 6.13. Sample admittance parameters (imaginary part) - example $2 \ldots \ldots 167$

Fig. 6.14. Transient responses - example $2 \ldots \ldots \ldots \ldots \ldots \ldots \ldots \ldots \ldots \ldots \ldots \ldots \ldots \ldots$

Fig. 7.1. A typical signal path consisting of diverse high-speed modules $\ldots \ldots 171$

Fig. 7.2. RLC circuit with an embedded state-space module . . . . . . . 175

Fig. 7.3. Eigenvalue of $\operatorname{Real}(\boldsymbol{Y}(j \omega)) \mathrm{v} / \mathrm{s}$ frequency $\ldots \ldots \ldots \ldots \ldots \ldots \ldots$ 
Fig. 7.4. Eigenvalue of $\operatorname{Real}(\boldsymbol{Y}(j \omega)) \mathrm{v} / \mathrm{s}$ frequency $\ldots \ldots \ldots \ldots \ldots \ldots \ldots$

Fig. 7.5. Pseudocode for the proposed global model-order reduction algorithm (PRESS) . . . . . . . . . . . . . . . . . . . 185

Fig. 7.6. Eigenvalue of $\operatorname{Real}(\boldsymbol{Y}(j \omega))$ - example $1 \ldots \ldots \ldots \ldots \ldots \ldots \ldots$

Fig. 7.7. Eigenvalue of $\operatorname{Real}(\boldsymbol{Y}(j \omega))$ - example $1 \ldots \ldots \ldots \ldots \ldots \ldots \ldots$

Fig. 7.8. Circuit with lumped components and measured subnetwork

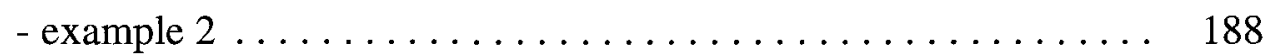

Fig. 7.9. Frequency responses - example $2 \ldots \ldots \ldots \ldots \ldots \ldots \ldots \ldots$

Fig. 7.10. Eigenvalues of $\operatorname{Real}(\boldsymbol{Y}(j \omega)) \mathrm{v} / \mathrm{s}$ frequency for the reduced system

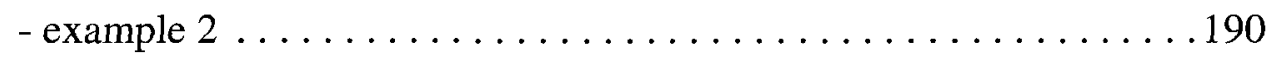

Fig. 7.11. Time domain responses - example $2 \ldots \ldots \ldots \ldots \ldots \ldots \ldots \ldots \ldots$

Fig. 7.12. Circuit of Example 3............................ 193

Fig. 7.13. Eigenvalue spectrum of $\operatorname{Real}(\boldsymbol{Y}(j \omega))$ : Original and reduced

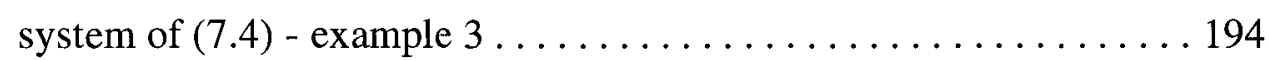

Fig. 7.14. Eigenvalue spectrum of $\operatorname{Real}(\boldsymbol{Y}(j \omega))$ : Original vs. PRESS - example 3

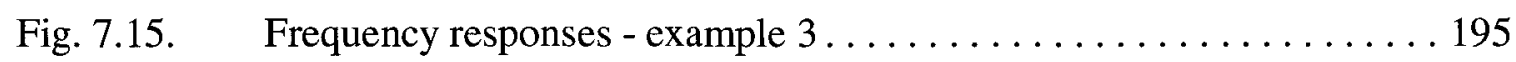

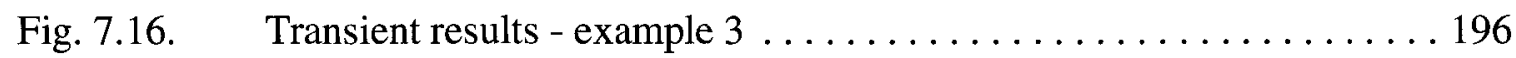

Fig. 7.17. Pseudocode for the proposed second-level reduction algorithm (FPTBR) for generating compact passive macromodels ........ 202

Fig.7.18. Subnetwork consisting of diverse linear modules ............ 203

Fig. 7.19. Comparison of transient response.................... 204

Fig. 7.20. Subnetwork consisting of diverse high-speed modules ......... 206

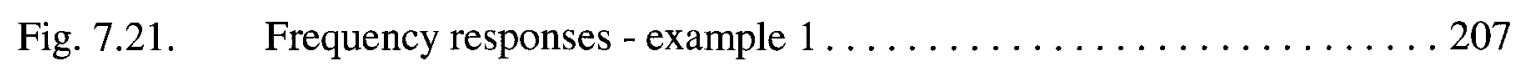

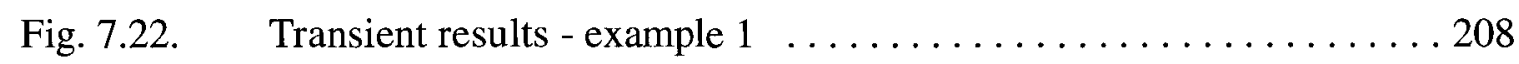

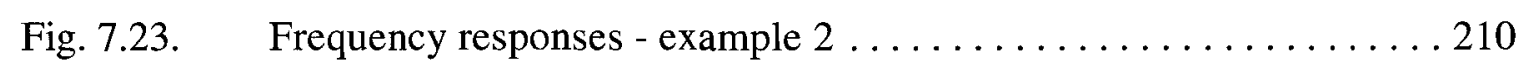

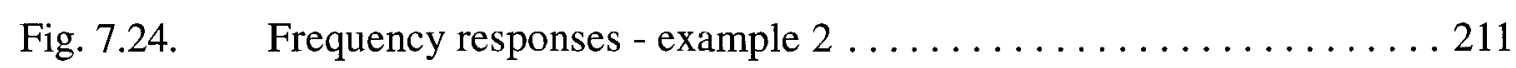

Fig. 7.25. Transient responses - example $2 \ldots \ldots \ldots \ldots \ldots \ldots \ldots \ldots \ldots \ldots \ldots \ldots \ldots \ldots$

Fig. B.1. Perturbation of imaginary eigenvalues ................. 224 


\section{List of Tables}

Table. 5.1. Comparison of computational complexity of passivity verification. . . 122

Table. 5.2. Summary of CPU times for passivity enforcement using PCFOP algorithm in conjunction with different verification methods and full Lyapunov solution - example $2 \ldots \ldots \ldots \ldots \ldots \ldots \ldots \ldots \ldots \ldots$

Table. 5.3. Summary of CPU times for passivity enforcement using PCFOP algorithm in conjunction with different verification methods and proposed fast Lyapunov solution - example $2 \ldots \ldots \ldots \ldots \ldots$

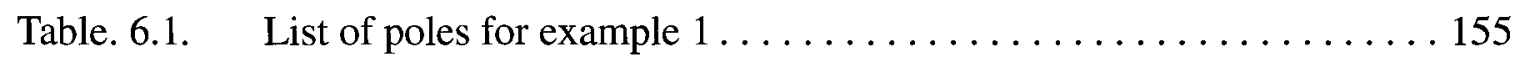

Table. 6.2. Details of proposed passivity correction algorithm for example $1 \ldots 156$

Table. 6.3. Details of proposed passivity correction algorithm for example $2 \ldots 165$

Table. 7.1. Comparison of CPU time in sec. - example $2 \ldots \ldots \ldots \ldots \ldots \ldots$

Table. 7.2. Comparison of CPU time in sec. - example 3.............. 193

Table. 7.3. Comparison of CPU time in sec. - example $1 \ldots \ldots \ldots \ldots \ldots \ldots$

Table. 7.4. Comparison of CPU time in sec. - example 2. . . . . . . . . . 209 


\section{Glossary of Terms}

ARE

. Algebraic Riccati Equation

AWE

Asymptotic Waveform Evaluation

BCFH . Block Complex Frequency Hopping

CAD . Computer Aided Design

$\mathrm{CFH}$ . Complex Frequency Hopping

CPU . Central Processing Unit

EM . Electromagnetic

FPTBR . Fast Positive Real Truncated Balanced Realization

GMOR . Global Model Order Reduction

IFFT . Inverse Fast Fourier Transform

$\mathrm{KCL}$ . Kirchoff's Current Law

LMI . Linear Matrix Inequality

LMOR. Local Model Order Reduction

MMT. Moment Matching Technique

MNA Modified Nodal Analysis

PCB . Printed Circuit Board

PR Positive Real

PRESS. . Passive Reduction Algorithm for RLC Interconnect Circuits with Embedded State-Space Systems

PRIMA Passive Reduced Order Interconnect Macromodel Algorithm

PRTBR Positive Real Truncated Balanced Realization

SPICE Simulation Program with Integrated Circuits Emphasis

TBR . Truncated Balanced Realization

VLSI Very Large Scale Integrated Circuit 


\section{CHAPTER 1}

\section{Introduction}

\subsection{Background and Motivation}

The rapid growth in microwave and VLSI technology coupled with the ever increasing quest for high-speed applications is placing enormous demands on computer-aided design (CAD) tools focussed on high-frequency modules. The design requirements are becoming very stringent, demanding sharper excitations, denser layouts, integration of analog and digital blocks and lower power consumption. This has highlighted the previously neglected high-frequency interconnect effects such as reflections, crosstalk, ringing, signal distortion and delay [1] - [5] (see Fig. 1.1). These effects, if not predicted at early design stages can severely degrade the system performance and thus render the fabricated circuit inoperable. It is therefore, important for the designers to simulate the interconnect effects as efficiently and accurately as possible [5] - [15].

However, transient analysis of interconnect circuits suffer from two major bottlenecks. First, interconnect circuits may consist of large number of components, such as resistors, capacitors, inductors, transmission lines and other high-frequency embedded modules, leading to excessive CPU time for transient analysis. Secondly, terminations of interconnect circuits generally consist of nonlinear elements such as drivers and receivers, which are described in time- 
domain. On the other hand, interconnects are best described in frequencydomain. Such a mixed frequency/time formulation cannot be handled appropriately by a traditional ordinary differential equation solvers, such as SPICE. During the recent years, these difficulties led to intense research in the development of efficient transient simulation algorithms for accurate and efficient simulation of interconnect networks [6] - [15].

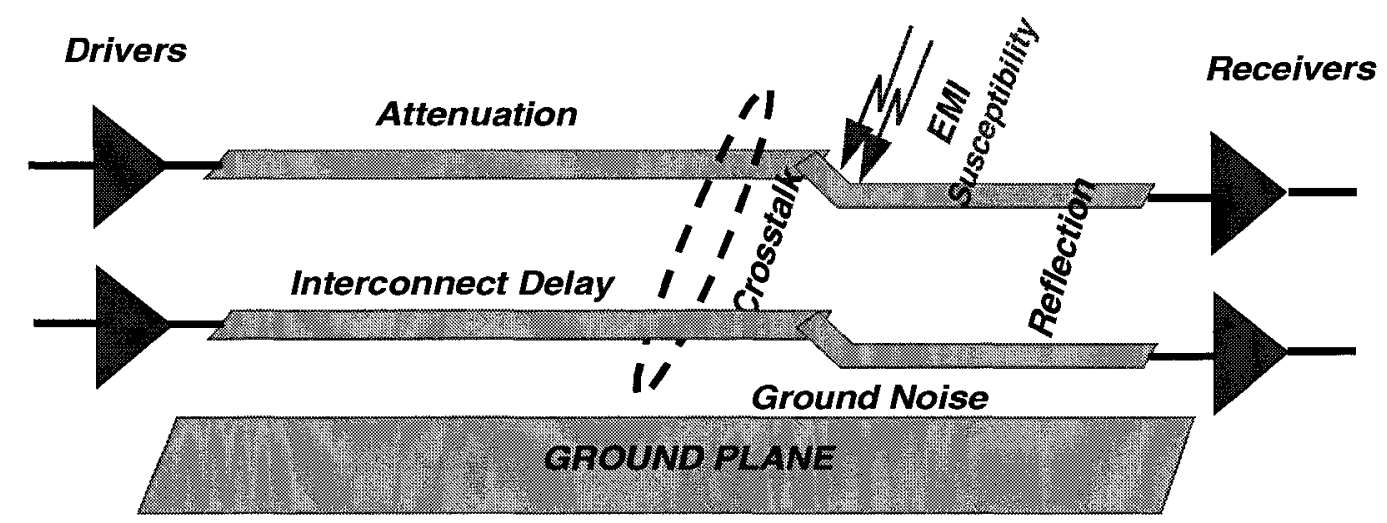

Fig. 1.1. High-speed interconnect effects

One of the highly focussed field in the analysis of high-speed modules, is the modeling and characterization based on tabulated data [16] - [57]. It is fast becoming an integral part of signal integrity validations, due to the diverse and complex nature of high-speed modules in microwave and integrated circuit designs. The tabulated data can be in the form of admittance $(\mathrm{Y})$, impedance $(\mathrm{Z})$, scattering $(\mathrm{S})$ or hybrid $(\mathrm{T})$ parameters, and can be obtained from either measurements [58] or rigorous electromagnetic simulations [20]. Important applications of such a characterization include high-speed interconnects, packages, vias, connectors, nonuniform transmission lines and on-chip passive components, such as inductors and transformers. However, transient simulation of such frequency-dependent tabulated data in the presence of nonlinear devices to obtain a global electrical assessment is a CPU expensive process due to the 
mixed frequency/time problem. Even the state-of-the-art commercial simulators such as [59] - [62], do not adequately handle the tabulated networks. As a result, this issue has become an area of intense research leading to several algorithms [20] - [57] during the recent years.

These algorithms essentially approximate the frequency-domain tabulated data by rational functions [20], [23], [26], [29] - [31], [37], and then convert them to state-space macromodels for transient analysis purposes. Some of the recent algorithms for this purpose [21], [22] use the low frequency physically intuitive models and perturb their characteristics to match the overall frequency spectrum of interest. A key challenge faced by the above mentioned algorithms is to ensure the passivity of the resulting macromodel. Passivity is an important property to be satisfied, because transient simulations of nonpassive macromodels may encounter artificial oscillations. Algorithms based on convex optimization [34], [35] although can generate passive macromodels, but are still CPU expensive for practical purposes. Alternative approaches use constraints such as, every first or second-order pole-residue pair must strictly conform to passivity relations (which is sufficient but not necessary), can be an over-treatment of the problem [38], [46].

Other important class of algorithms [27], [28], [39] - [43] first attempt to get a nearly passive macromodel and then compensate for any loss of passivity using first-order perturbation techniques. Passivity enforcement in these techniques is worked upon iteratively, with each iteration involving passivity verification and compensation for any passivity violation. The major challenges faced by these algorithms are the issues of passivity verification, identification and quantification of passivity violating regions, and an adequate strategy for the passivity enforcement. In addition, these algorithms scale very badly with large scale nonpassive macromodels [47], [53]. This is because, for large sized systems 
Hamiltonian based passivity checking/compensation requires considerable CPU time. Since this is required to be performed in every iteration it can cause the overall passivity enforcement process to be prohibitively CPU expensive. Also, an additional concern with these algorithms is that, the passivity correction process does not have a guaranteed direction. In other words, the passivity compensation at one frequency point may lead to passivity violation at some other frequency points, which in turn will have to be corrected for passivity. This increases the number of iterations required for the complete passivity enforcement process, leading to increased CPU cost.

In addition, as a result of the growing trend of describing interconnect networks by tabulated data, a modern design can consist of signal paths comprising of diverse high-speed modules such as, large lumped RLC subcircuits, distributed interconnects (whose analytical models are available) and tabulated modules (see Fig. 1.2). Unified transient analysis of such signal paths of diverse modules is associated with the following two major issues:

1) Transient analysis of such a signal path is highly CPU intensive because of the large number of equations governing the unified circuit. For an efficient transient simulation, it is desired to obtain a single reduced macromodel for the entire signal path. However, the existing algorithm for model reduction such as, PRIMA are applicable only to the RLC type of circuits or circuits whose MNA matrices conform to positive semi-definite conditions. But, the unified matrices of the signal path with diverse modules do not necessarily satisfy these conditions. Currently, there is no algorithm available which can ensure a global passive model reduction of such a system. 

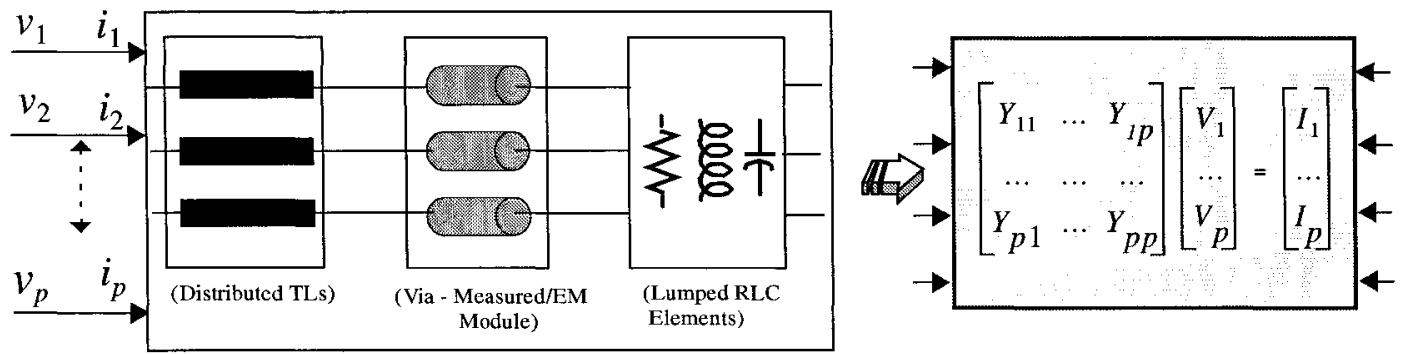

Fig. 1.2. Linear subnetwork with diverse high-speed modules (distributed, EM, RLC elements etc.)

2) The second issue is that, PRIMA type of first-level reduced order models are still of high order and not compact enough (thereby result in excessive CPU time during transient simulation). This observation has led to the focus on second-level reduction algorithms such as, truncated balanced realization (TBR) [71]. To ensure the passivity of the reduced system from TBR, algorithms such as [72], [73] have been proposed. However, one of the issues with these algorithms is the associated high computational cost.

In order to address the above issues, which are highly challenging the design automation and signal integrity community, the contributions of this thesis are given in the next section.

\subsection{Contributions}

In this thesis, novel and fast algorithms are developed to address the issue of including high-speed modules in circuit simulators and model reduction algorithms. The specific contributions are listed below.

1) A novel and robust algorithm is presented for the passive macromodeling of networks characterized by tabulated data [28], [27], [39], [40], [47], [49] - [52]. 
This algorithm presents a systematic approach for passivity checking, identification and quantification of passivity violating regions. For passivity compensation, two different strategies are presented: (a) an algorithm based on first-order perturbation of the residues of rational function approximation, (b) an algorithm employing linear matrix inequality (LMI) formulation, which can be solved efficiently by interior point methods.

2) A fast algorithm is presented for the passivity verification and compensation of large sized macromodels of networks characterized by tabulated data [47], [53]. The proposed algorithm combines the merits of the recent fast Hamiltonian eigensolvers [54], [55], [63] with the newly developed fast frequency sweep based on reciprocal system formulation. This leads to minimized number of Hamiltonian eigen evaluations during the iterative passivity compensation process of large-order macromodels, leading to computational speed-up. In addition, a method for the fast solution of Lyapunov equation of the state-space system is presented, which is required by passivity enforcement algorithms to maintain the macromodel accuracy during the compensation process.

3) A new global passivity enforcement algorithm is presented [56], [57] for subnetworks described by admittance parameters. This algorithm adopts a global approach for passivity enforcement by ensuring that the passivity correction at a certain region does not introduce new passivity violations at other parts of the frequency spectrum.

4) A novel algorithm is presented for the passive model-order reduction of RLC interconnect circuits with embedded state-space systems (PRESS) [64], [65], [66]. The proposed algorithm adopts a PRIMA type of formulation for RLC networks while providing a means to include multiple passive state- 
space described modules. Reduction is performed using congruent transformation to obtain a single passive macromodel for the entire linear subnetwork.

5) A new method [65], [66] is presented to reduce the computational cost of the second-level passive reduction algorithm based on truncated balanced realization (TBR). The major advantage of the algorithm is that, it obtains the solution of two Lur e equations by solving only one equation and an inexpensive Sylvester equation. As a result, it cuts the computation cost by almost half as compared to the existing second-level reduction algorithms.

\subsection{Organization of the Thesis}

This thesis is organized as follows. In chapter 2, a survey of the macromodeling/ simulation algorithms for passive subnetworks characterized by tabulated data is presented. Chapter 3 reviews the model reduction algorithms based on direct moment-matching (AWE) and indirect moment-matching (PRIMA). This chapter also discusses the TBR procedure for the reduction of state-space systems. Chapter 4 presents a novel and robust algorithm for the macromodeling of tabulated data for small/medium sized models. In chapter 5 , an efficient algorithm is presented for the passive macromodeling of tabulated networks with large sized models. In chapter 6 , a new global passivity enforcement algorithm for subnetworks described by admittance parameters is presented. Chapter 7 presents a new global model-order reduction (GMOR) scheme. It combines the novel algorithm for passive model-order reduction of RLC interconnect circuits with embedded passive state-space systems (PRESS) and the proposed secondlevel reduction algorithm with reduced computation cost. A summary of this thesis work and discussion of the future work is presented in chapter 8 . 


\section{CHAPTER 2}

\section{Review of Modeling/Simulation of Tabulated Data Subnetworks}

As discussed in chapter 1, with the ever increasing operating frequency and complexity, it may not always be possible to find an analytical model for the interconnect structures due to complex geometries and high-frequency requirements [23] - [57]. In such cases, they are generally characterized by frequency-domain tabulated data obtained from terminal measurements or fullwave analysis. Subnetworks described by tabulated data are becoming an integral part of modern high-speed designs. However, transient simulation of such frequency-dependent tabulated data in the presence of nonlinear devices to obtain a global electrical assessment is a CPU expensive process due to the mixed frequency/time problem. Prominent approaches to solve this difficulty [33] - [52] are based on approximating the tabulated data through rational functions and subsequently synthesizing a SPICE compatible macromodel/netlist from such an approximation. However, the primary challenge in such approaches is ensuring the passivity of the macromodel. Passivity is an important property, because stable but non-passive models may lead to unstable systems when connected to other passive components.

The objective of this chapter is to lay the foundation for understanding the work presented in this dissertation. It reviews some of the prominent techniques for including frequency sampled data in the overall circuit simulation. This includes 
techniques based on the direct convolution of tabulated data with the terminations, as well as the rational function based approaches.

\subsection{Modeling/Simulation of Subnetworks Characterized by Tabulated Data}

Modeling and simulation of subnetworks characterized by tabulated data has become an indispensable part of the signal integrity analysis of modern highspeed designs. Several algorithms have been suggested for this purpose during the recent years. Fig. 2.1 shows an overview of generally adopted flow for modeling/simulation of tabulated subnetworks in a transient analysis. In a broader perspective, there are two ways of handling tabulated data in a transient analysis. The most straight forward approach is to perform inverse Fourier transform (IFFT) on the frequency-domain tabulated data to obtain its timedomain counterpart and then perform convolution with the time-domain behaviour of the terminations and sources.

The second approach is based on obtaining a macromodel of the tabulated data, via rational function approximation. One of the major challenges in this approach is ensuring the passivity of the resulting macromodel. Passivity is an important property to be satisfied, because non-passive models may lead to unstable systems when connected to other passive components. Once a passive macromodel is obtained, it can be included in the time-domain simulator as ordinary differential equations.

In the following sections, the above mechanisms of handling tabulated data are described in more detail. Section 2.2 describes the convolution based approach, while Section 2.3 discusses the macromodeling approaches based on rational 
function approximations. Section 2.4 - Section 2.5 discuss the issue of the passivity and passivity guaranteeing algorithms respectively.

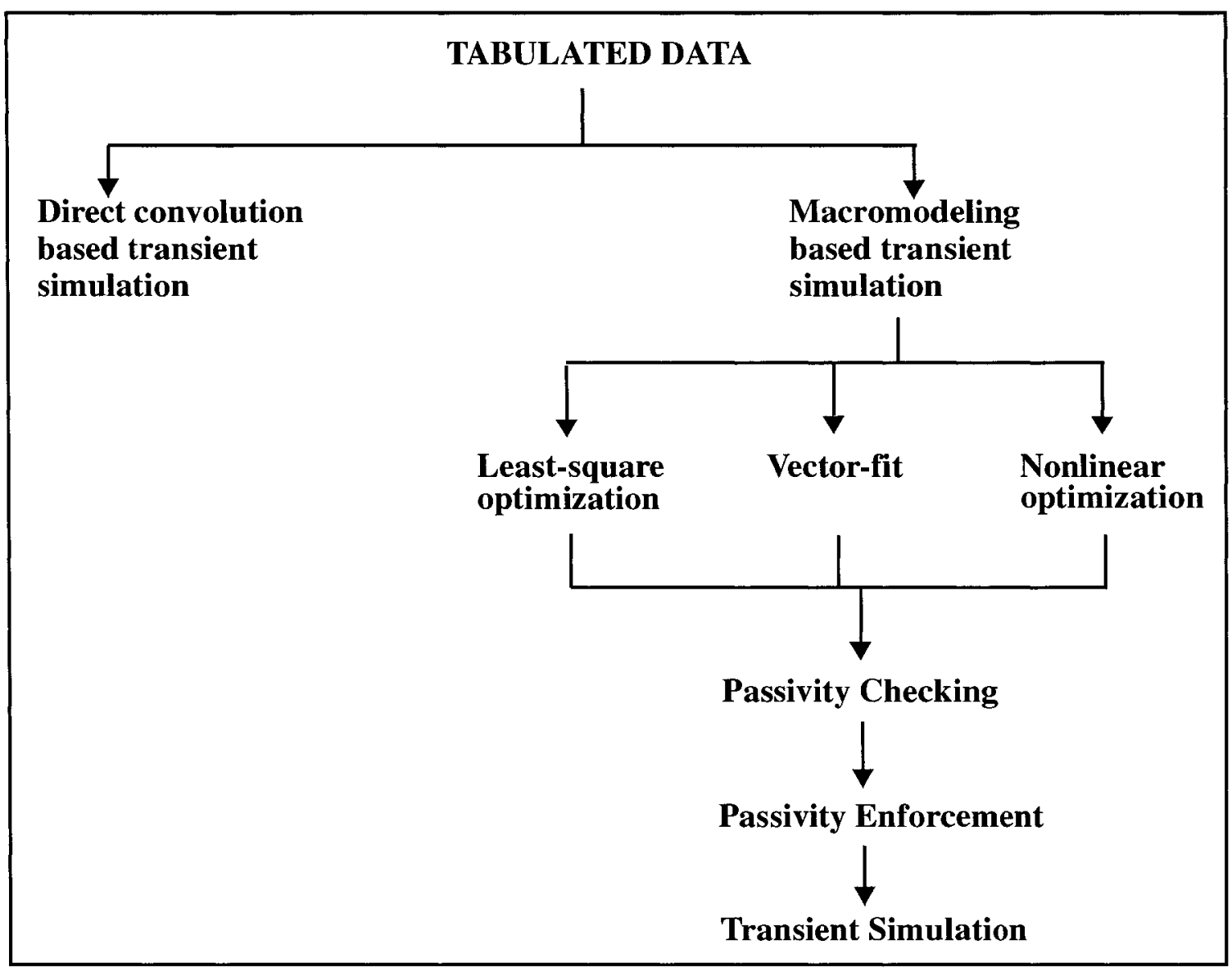

Fig. 2.1. Commonly adopted modeling/simulation flow for tabulated networks

\subsection{Convolution Based Transient Analysis}

The convolution based method is the most straight forward way of including frequency-domain tabulated data in a transient analysis. In this method, transient simulation is performed by first converting the frequency sampled data into time- 
domain using inverse Fourier transform (IFFT) and then convolving it with the input excitation [16] - [18]. The details of this technique are given below.

Consider a linear, passive M-port network of Fig. 2.2. Suppose that an ideal voltage source, $v_{j}(t)$, is connected at port $j$, while other ports are short circuited. Then the currents at all the ports can be represented as

$$
I_{k}(\omega)=Y_{k j}(\omega) V_{j}(\omega), \quad k=1,2, \ldots M
$$

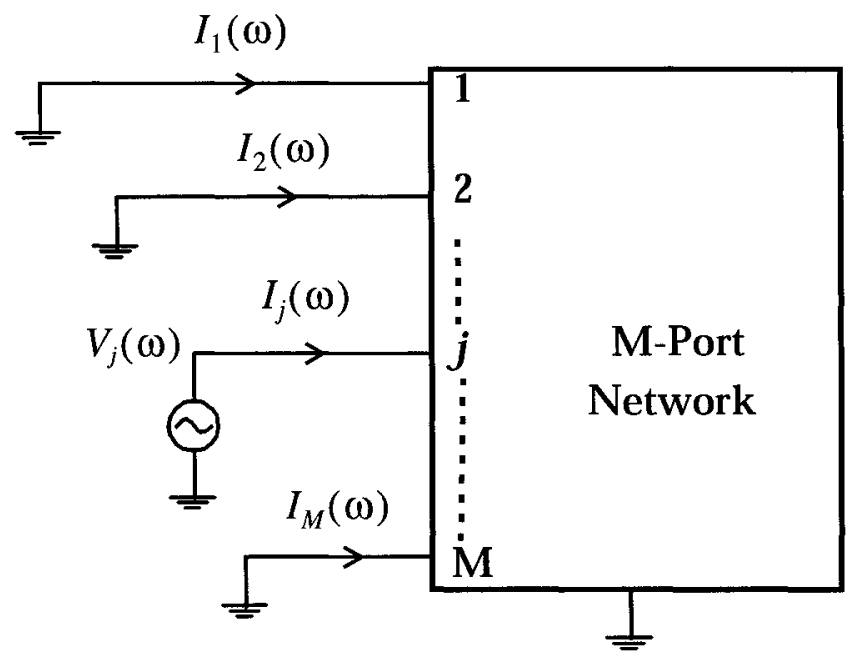

Fig. 2.2. M-port network

where, $V_{j}(\omega)$ is the Fourier transform of $v_{j}(t)$ and $Y_{k j}(\omega)$ are the network $Y$ parameters relating port $j$ to the port $k$, while $\omega$ is the angular frequency. For a specific case of $v_{j}(t)$ being a unit delta function, $V_{j}(\omega)=1$, which is independent of frequency, the port currents in time-domain can be obtained as

$$
i_{k}(t)=i_{g k j}(t)=F^{-1}\left\{Y_{k j}(\omega)\right\}
$$


where $F^{-1}$ denotes the inverse Fourier transform. These currents $i_{g k j}(t)$ are referred to as the Green s functions of the network. When $v_{j}(t)$ is an arbitrary function, the port currents can be obtained as

$$
i_{k}(t)=F^{-1}\left\{Y_{k j}(\omega) V_{j}(\omega)\right\}=i_{g k j}(t) * v_{j}(t)=\int_{0}^{t} i_{g k j}(t-\tau) v_{j}(\tau) d \tau
$$

where ${ }^{*}$ denotes the convolution, and is represented by its integral form, assuming that all the excitations begin after $t=0$. By the superposition principle for linear networks, the current at port $k$ in the presence of applied voltages at all the ports can be written as

$$
i_{k}(t)=\sum_{j=1}^{M} \int_{0}^{t} i_{g k j}(t-\tau) v_{j}(\tau) d \tau
$$

The voltages at ports with non-linear terminations may be evaluated by substituting $i_{k}(t)$ in the specific voltage-current relationship defined by the terminating nonlinear network. For instance, for a diode connected at port $k$, the voltage-current relationship can be expressed as

$$
i_{k}(t)=I_{s}\left(\exp \left(\frac{v_{k}(t)}{V_{T}}\right)-1\right)
$$

Notice that the network $Y$-parameters $Y_{k j}(\omega)$ are computed at discrete frequency points, by connecting an ideal impulse (delta) function at a port, short-circuiting all other ports and then finding the currents at each port. Later, the inverse Fourier transform of $Y_{k j}(\omega)$ is evaluated as indicated in (2.2). In order to prepare (2.4) for practical implementation, the integrations are replaced by summations as follows 


$$
i_{k}\left(t_{q}\right)=\sum_{j=1 p=0}^{M} \sum_{i_{k j}}^{q}\left(t_{q}-t_{p}\right) v_{j}\left(t_{p}\right) \Delta t, \quad k=1,2, \ldots M
$$

where $\Delta t$ represents the time step and $t_{q}, t_{p}$ denote the time instances $q \Delta t, p \Delta t$ respectively.

As pointed out in [16], [18], the frequency-domain analysis is done numerically, at a finite number of discrete frequencies. In the time-domain, the Green s function $i_{g k j}$ in (2.2) must also be discretized. In addition, this function must be convolved with port voltages, which is also done numerically. Since convolution is an expensive process, it is desired that the number of samples of $i_{g k j}$ is kept as low as possible. This can be a problem if the response of the network with terminations in place, spans a time interval greater than a few transit times of the network (transit time of a network is the time delay associated with the signal propagation in the network). To overcome this problem, for the case of transmission lines, it has been suggested in [16] - [18], to terminate the lines with well-matched networks while evaluating the transfer functions $Y_{k j}(\omega)$. This reduces the duration of function $i_{g k j}$ to only a few line transit times. As a result, the convolution in (2.6) can be made less expensive. For the guaranteed convergence of convolution based methods, it is desired that the frequency-domain data of the admittance matrix $\boldsymbol{Y}(j \omega)$ should satisfy the positive-real criteria at the given frequency points.

Although the convolution based approach seems straight forward, it is associated with several major difficulties [45]. Firstly, the number of time points required for the desired accuracy may be very high. This leads to the evaluation of (2.4) to be CPU expensive. Secondly, the IFFT used to transform the frequency-domain data into time-domain requires special attention to avoid aliasing. Extrapolation and 
low-pass filtering of the frequency-domain data are required to reduce the timedomain ripple associated with IFFT. Further, evaluation of (2.4) becomes computationally very expensive for interconnect networks with large number of ports.

A more efficient method of incorporating the tabulated data into the circuit simulator is by representing it by rational functions in complex variable $s^{\prime}[26]$, [45]. This avoids the difficulties encountered by the direct convolution based techniques indicated above. The rational functions of $s^{\prime}$ can be incorporated into time-domain simulators by either converting them into a set of ordinary differential equations or using recursive convolution [19]. Some of the important algorithms based on rational function approximations for tabulated data, are described in the next section.

\subsection{Rational Function Based Approaches}

One of the most common ways of representing frequency-domain tabulated data of electrical responses is approximating it by rational functions in complex variable $s$. This idea has been utilised by many researchers [20], [23], [26], [29] [31], [37], [44] - [46] for representing the behaviour of interconnect networks. The underlying objective in these approaches is to approximate the frequency-domain data $\left\{Y\left(s_{1}\right), Y\left(s_{2}\right), \ldots Y\left(s_{N}\right)\right\}$ given at $N$ frequency points, by a rational function of polynomials in $s^{\prime}$

$$
Y(s)=\left.\frac{a_{0}+a_{1} s+a_{2} s^{2}+a_{3} s^{3}+\ldots+a_{m-1} s^{m-1}+a_{m} s^{m}}{1+b_{1} s+b_{2} s^{2}+b_{3} s^{3}+\ldots+b_{n-1} s^{n-1}+b_{n} s^{n}}\right|_{s=s_{k} ; \quad\{k=1,2, \ldots, N\}}
$$


and obtain the unknown coefficients $a_{0}, a_{1} \ldots a_{m}$ and $b_{1}, b_{2} \ldots b_{n}$. Equation (2.7) is a nonlinear function of the unknown coefficients, but can be converted into the standard linear least-square formulation $\boldsymbol{A} \boldsymbol{x}=\boldsymbol{b}$ by multiplying both sides by the denominator polynomial. However, the resulting formulation is badly conditioned and may present serious computational challenges in obtaining highorder approximations for broad-band representations. Several methods to overcome this problem have been suggested. Methods such as those in [29] and [30] propose various scaling schemes to improve the conditioning of the problem. However, these techniques may not work for badly scaled problems resulting from high-order approximations. Iterative techniques such as [26] and [37] have also been proposed to overcome the problem of ill-conditioning in approximating the tabulated data. The following subsections describe various rational function based approaches.

\subsubsection{Standard Least-Square}

Standard least-square technique is the most common way of representing the tabulated data by rational approximation of the form (2.7). Unknown coefficients $a_{0}, a_{1} \ldots a_{m}$ and $b_{1}, b_{2} \ldots b_{n}$ are obtained by multiplying both sides of (2.7) by the denominator polynomial resulting in the following equation

$$
a_{0}+a_{1} s+a_{2} s^{2}+\ldots+a_{m-1} s^{m-1}+a_{m} s^{m}=Y(s)\left(1+b_{1} s+b_{2} s^{2}+\ldots+b_{n-1} s^{n-1}+b_{n} s^{n}\right)
$$

When written at $N$ frequency points, (2.8) results in the following matrix equation 


$$
\left[\begin{array}{ccccccccc}
1 & s_{1} & s_{1}^{2} & \ldots & s_{1}^{m} & -s_{1} Y\left(s_{1}\right) & -s_{1}^{2} Y\left(s_{1}\right) & \ldots & -s_{1}^{n} Y\left(s_{1}\right) \\
1 & s_{2} & s_{2}^{2} & \ldots & s_{2}^{m} & -s_{2} Y\left(s_{2}\right) & -s_{2}^{2} Y\left(s_{2}\right) & \ldots & -s_{2}^{n} Y\left(s_{2}\right) \\
\vdots & \vdots & \vdots & \vdots & \vdots & \vdots & \vdots & \vdots & \vdots \\
1 & s_{N} & s_{N}^{2} & \ldots & s_{N}^{m} & -s_{N} Y\left(s_{N}\right) & -s_{N}^{2} Y\left(s_{N}\right) & \ldots & -s_{N}^{n} Y\left(s_{N}\right)
\end{array}\right]\left[\begin{array}{c}
a_{0} \\
a_{1} \\
a_{2} \\
\vdots \\
a_{m} \\
b_{1} \\
b_{2} \\
\vdots \\
b_{n}
\end{array}\right]=\left[\begin{array}{c}
Y\left(s_{1}\right) \\
Y\left(s_{2}\right) \\
\vdots \\
Y\left(s_{N}\right)
\end{array}\right]
$$

Equation (2.9) involves real as well as the imaginary parts of $Y(s)$. In practice, the real part of $Y(s)$ is approximated by a rational function to evaluate the poles of the system [29]. The real part of network function $Y(s)$ can be approximated with the real rational polynomial function of the squared variable [29] as follows

$$
\operatorname{Re}\{Y(s)\}=Y^{r}(s)=\frac{c_{0}+c_{1} s^{2}+c_{2} s^{4}+\ldots+c_{m} s^{2 m}}{1+d_{1} s^{2}+d_{2} s^{4}+\ldots+d_{n} s^{2 n}}
$$

Since the poles of the even function of $Y(s)$ are those of both $Y(s)$ and $Y(-s)$, those belonging to $Y(s)$ lie in the left-half plane [29], [45]. Thus the denominator of (2.7) can be found from (2.10). The following system of equations result by writing (2.10) at $N$ number of frequency points and substituting $s=j \omega$. 


$$
\left[\begin{array}{ccccccccc}
1 & -\omega_{1}^{2} & \omega_{1}^{4} & \ldots & (-1)^{m} \omega_{1}^{2 m} & \omega_{1}^{2} Y^{r}\left(j \omega_{1}\right) & -\omega_{1}^{4} Y^{r}\left(j \omega_{1}\right) & \ldots & (-1)^{n+1} \omega_{1}^{2 n} Y^{r}\left(j \omega_{1}\right) \\
1 & -\omega_{2}^{2} & \omega_{2}^{4} & \ldots & (-1)^{m} \omega_{2}^{2 m} & \omega_{2}^{2} Y^{r}\left(j \omega_{2}\right) & -\omega_{2}^{4} Y^{r}\left(j \omega_{2}\right) & \ldots & (-1)^{n+1} \omega_{2}^{2 n} Y^{r}\left(j \omega_{2}\right) \\
\vdots & \vdots & \vdots & \vdots & \vdots & \vdots & \vdots & \vdots & \vdots \\
1 & -\omega_{N}^{2} & \omega_{N}^{4} & \ldots & (-1)^{m} \omega_{N}^{2 m} & \omega_{N}^{2} Y^{r}\left(j \omega_{N}\right) & -\omega_{N}^{4} Y^{r}\left(j \omega_{N}\right) & \ldots & (-1)^{n+1} \omega_{N}^{2 n} Y^{r}\left(j \omega_{N}\right)
\end{array}\right]\left[\begin{array}{c}
c_{0} \\
c_{1} \\
c_{2} \\
\vdots \\
c_{m} \\
d_{1} \\
d_{2} \\
\vdots \\
d_{n}
\end{array}\right]=\left[\begin{array}{c}
Y^{r}\left(j \omega_{1}\right) \\
Y^{r}\left(j \omega_{2}\right) \\
\vdots \\
Y^{r}\left(j \omega_{N}\right)
\end{array}\right]
$$

or

$$
\boldsymbol{W} \boldsymbol{X}=\boldsymbol{Z}
$$

where $\boldsymbol{W} \in \mathfrak{R}^{N \times q}, \boldsymbol{X} \in \mathfrak{R}^{q \times 1}, \boldsymbol{Z} \in \mathfrak{R}^{N \times 1}$ and $q=n+m+1$. For a deterministic solution of (2.12), the number of frequency points, $N$, should be greater than or equal to the number of unknowns, $q$. Equation (2.12) can be solved using the standard Householder algorithm, i.e. $\boldsymbol{R} \boldsymbol{X}=\boldsymbol{Q}^{T} \boldsymbol{Z}$, where $\boldsymbol{W}=\boldsymbol{Q R}, \boldsymbol{Q}$ is an orthogonal matrix and $\boldsymbol{R}$ is an upper triangular matrix [29]. The coefficients of the denominator of $Y(s)$ are obtained by solving (2.12) for $\boldsymbol{X}$.

Subsequently, the denominator polynomial is factorized to obtain the poles. Any unstable or purely imaginary poles are ignored. Hence, the order of the approximating rational function should be set greater than or equal to the actual order sought while formulating (2.11). 


\subsection{1a Computation of Residues}

Once the poles of the system are known, $Y(s)$ can be written in the pole-residue form as

$$
Y(s)=c+\sum_{i=1}^{n^{\prime}} \frac{k_{i}}{s-p_{i}}
$$

where $c$ is the direct coupling constant, $k_{i}$ are residues and $p_{i}$ are the poles. Notice that $n^{\prime} \leq n$ and $n-n^{\prime}$ is the number of rejected unstable/purely-imaginary poles. The unknowns to be solved in (2.13) are the residues $\left(k_{i}{ }^{\prime} s\right)$ and the direct coupling constant $c$. For this purpose, (2.13) is expressed at various frequency points to form a matrix least-square problem $\boldsymbol{A} \boldsymbol{X}=\boldsymbol{b}$, where $\boldsymbol{X}$ is the vector of unknowns. The unknowns here are $\left(c, k_{i}, i \in 1,2, \ldots n^{\prime}\right)$. The exact form of the matrix equation will depend on whether the poles are real or complex. The real part of (2.13) can be expressed as

$$
\operatorname{Re}\{Y(s)\}=c+\sum_{i=1}^{n_{R}} k_{i}\left[\operatorname{Re}\left\{\frac{1}{s-p_{i}}\right\}\right]+\sum_{i=n_{R}+1} \operatorname{Re}\left\{\frac{k_{i}}{\left\{s-p_{i}\right\}}+\frac{k_{i}^{*}}{\left\{s-p_{i}^{*}\right\}}\right\}
$$

and the imaginary part as

$$
\operatorname{Im}\{Y(s)\}=\sum_{i=1}^{n_{R}} k_{i}\left[\operatorname{Im}\left\{\frac{1}{s-p_{i}}\right\}\right]+\sum_{i=n_{R}+1} \operatorname{Im}\left\{\frac{k_{i}}{\left\{s-p_{i}\right\}}+\frac{k_{i}^{*}}{\left\{s-p_{i}^{*}\right\}}\right\}
$$

where $n_{R}$ is the total number of real poles, and $n_{C}$ is the total number of pairs of complex conjugate poles. By utilizing the fact that complex conjugate poles have complex conjugate residues, (2.14) can be further expressed as 


$$
\begin{aligned}
\operatorname{Re}\{Y(s)\}=c+\sum_{i=1}^{n_{R}} k_{i}\left[\operatorname{Re}\left\{\frac{1}{s-p_{i}}\right\}\right] & +\sum_{i=n_{R}+1} \operatorname{Re}\left\{k_{i}\right\} \operatorname{Re}\left\{\frac{1}{s-p_{i}}+\frac{1}{s-p_{i}^{*}}\right\} \\
& -\sum_{i=n_{R}+1}^{n_{R}+n_{C}} \operatorname{Im}\left\{k_{i}\right\} \operatorname{Im}\left\{\frac{1}{s-p_{i}}-\frac{1}{s-p_{i}^{*}}\right\}
\end{aligned}
$$

Similarly, (2.15) can be expressed as

$$
\begin{aligned}
\operatorname{Im}\{Y(s)\}=\sum_{i=1}^{n_{R}} k_{i}\left[\operatorname{Im}\left\{\frac{1}{s-p_{i}}\right\}\right] & +\sum_{i=n_{R}+1}^{n_{R}+n_{C}} \operatorname{Re}\left\{k_{i}\right\} \operatorname{Im}\left\{\frac{1}{s-p_{i}}+\frac{1}{s-p_{i}^{*}}\right\} \\
& +\sum_{i=n_{R}+1} \operatorname{Im}\left\{k_{i}\right\} \operatorname{Re}\left\{\frac{1}{s-p_{i}}-\frac{1}{s-p_{i}^{*}}\right\}
\end{aligned}
$$

Thus, by recasting (2.13) as (2.16) and (2.17) and then combining them into a single system of matrix equation of the form $\boldsymbol{A} \boldsymbol{X}=\boldsymbol{b}$, the unknown residues $\left(k_{i}\right)$ and the constant $c$ are evaluated. An example of the matrix equation that results from this procedure for a third order system with one real pole and one complex conjugate pair, is shown below. 


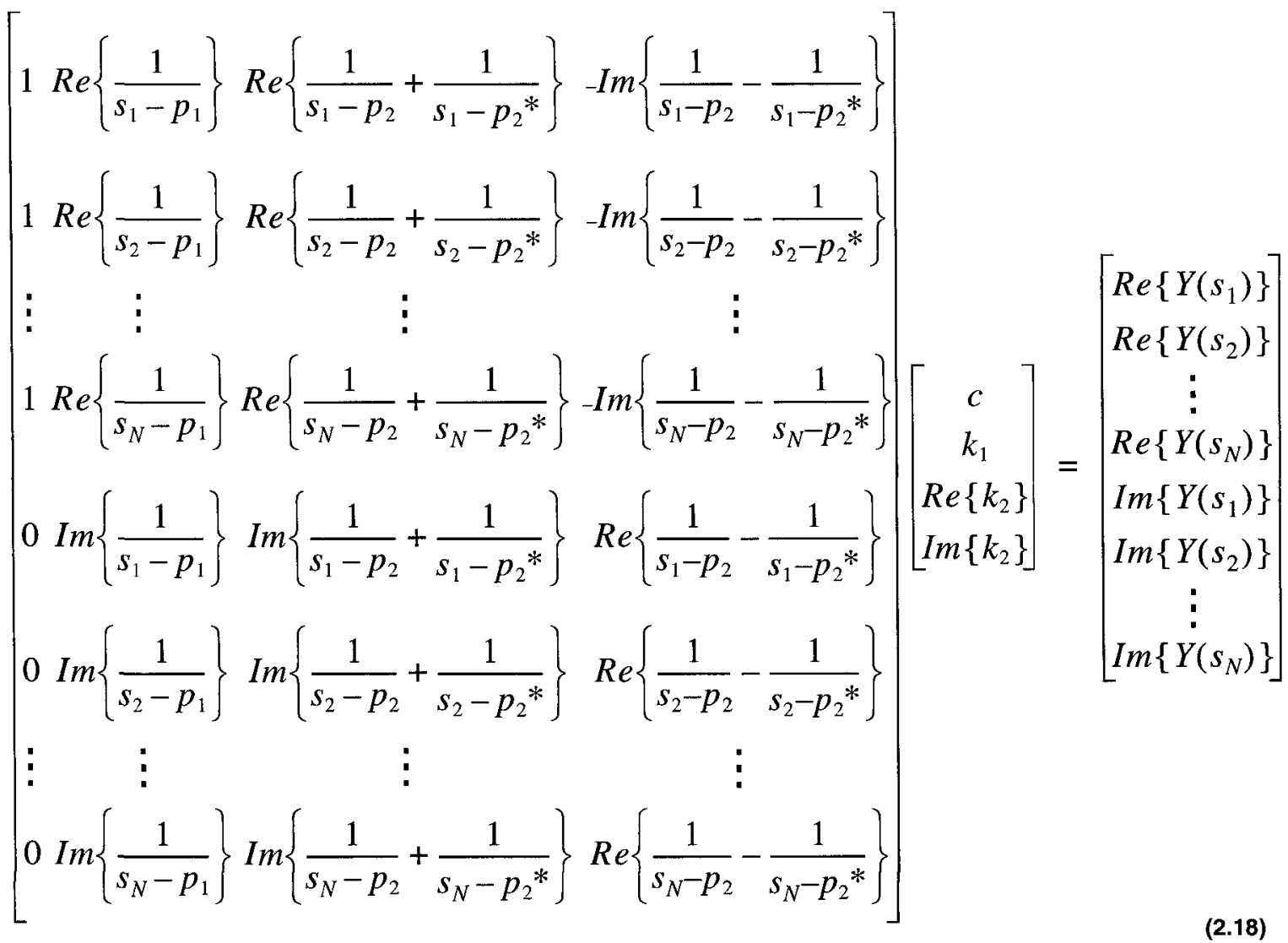

Here $N$ is the total number of frequency points. Once again, Householder leastsquare solver is used to solve the above matrix equation. After evaluating (2.18), the rational approximation of the transfer function (2.13) is known. This entire process is repeated for each element of the multiport transfer function matrix.

The above method works well for low-order approximations, but becomes illconditioned (system of (2.11)) for high-order approximations (required for spanning larger bandwidths). Some normalizing schemes for the radian frequency, $\omega$, have been suggested to overcome this problem. For instance, in [23] it is suggested to use the normalization factor $\omega_{\text {norm }}$ as

$$
\omega_{n o r m}=\sqrt{\omega_{\min } \cdot \omega_{\max }}
$$


before formulating (2.11), while in [29] two alternative schemes were suggested to overcome the problem. In the first one, the maximum frequency is normalized to unity, while the second scheme uses a shifted basis for the radian frequency $\omega$ using the transformation

$$
\omega^{\prime}=2 \frac{\left(\omega-\omega_{\min }\right)}{\left(\omega_{\max }-\omega_{\min }\right)}-1
$$

However, none of the above schemes completely eliminate the problem of illconditioning associated with the matrix in (2.11).

Recently, iterative algorithms such as vector-fit [26] and the nonlinear least-square [37] have been proposed to overcome the ill-conditioning associated with the above linear least-square technique. The next two subsections describe these two iterative algorithms.

\subsubsection{Vector Fitting by Pole Relocation}

Vector fitting algorithm approximates the given data $f(s)$ directly with the poleresidue approximation [26]

$$
f(s)=\sum_{i=1}^{n} \frac{k_{i}}{s-p_{i}}+c+s h
$$

where the residues $\left(k_{i}\right)$ and poles $\left(p_{i}\right)$ can be real or complex conjugate pairs, $n$ is the number of poles and residues, $c \& h$ are real. The problem is to estimate all the coefficients in (2.21) so that a least-squares approximation of $f(s)$ is obtained over a given frequency interval. Notice that this is a nonlinear problem in terms of unknowns, as $p_{i}$ appear in the denominator of (2.21). 
The vector fitting algorithm solves the problem in (2.21) through a linear formulation using two steps. In the first step, an initial guess of poles is made and the best poles are estimated iteratively. In the second step, the residues are calculated with the known poles. The second step is same as the computation of residues described in Section 2.3.1a. The ill-conditioning associated with the standard linear least-square approach is overcome as the matrices involved in both the steps of vector fitting algorithm involve entries with only $\omega$ (i.e. no higher powers of $\omega$ are involved). The details of the two steps of the vector fitting technique are described below.

\subsection{2a Pole Identification Algorithm}

An initial guess of poles $\bar{p}_{i}$ is specified and a scaling function $\sigma(s)$ of the following rational form is considered

$$
\sigma(s)=\sum_{i=1}^{n} \frac{\tilde{k}_{i}}{s-\bar{p}_{i}}+1
$$

Multiplying (2.22) with $f(s)$ of (2.21) we get a scaled function, $\sigma(s) f(s)$. Next, as shown below, this scaled function is approximated (represented by $\hat{f}(s)$ ) using the same set of poles as that of $\sigma(s)$

$$
\sigma(s) f(s) \approx \hat{f}(s)=\sum_{i=1}^{n} \frac{\hat{k}_{i}}{s-\bar{p}_{i}}+\hat{c}+s \hat{h}
$$

Using (2.22), (2.23) can be written as 


$$
\left[\sum_{i=1}^{n} \frac{\tilde{k}_{i}}{s-\bar{p}_{i}}+1\right] f(s) \approx\left[\sum_{i=1}^{n} \frac{\hat{k}_{i}}{s-\bar{p}_{i}}+\hat{c}+s \hat{h}\right]
$$

or

$$
\left[\sum_{i=1}^{n} \frac{\hat{k}_{i}}{s-\bar{p}_{i}}+\hat{c}+s \hat{h}\right]-\left[\sum_{i=1}^{n} \frac{\tilde{k}_{i}}{s-\bar{p}_{i}}\right] f(s)=f(s)
$$

In (2.25), $\bar{p}_{i}$ is the initial guess of poles and the total number of unknowns are $n_{t}=2 n+2$. Equation (2.25) when written at $N$ different frequency points $\left(N>n_{t}\right)$ results in an overdetermined linear problem of the form

$$
\boldsymbol{A X}=\boldsymbol{b}
$$

where

$$
\boldsymbol{b}=\left[\begin{array}{llll}
f\left(s_{1}\right) & f\left(s_{2}\right) & \ldots f\left(s_{N}\right)
\end{array}\right]^{T}
$$

The form of $\boldsymbol{A}$ and $\boldsymbol{X}$ depends on whether the poles are real or complex, as described below.

\section{Case 1: Real Poles}

In case of real poles, writing (2.25) at several frequency points results in the following matrix equation 


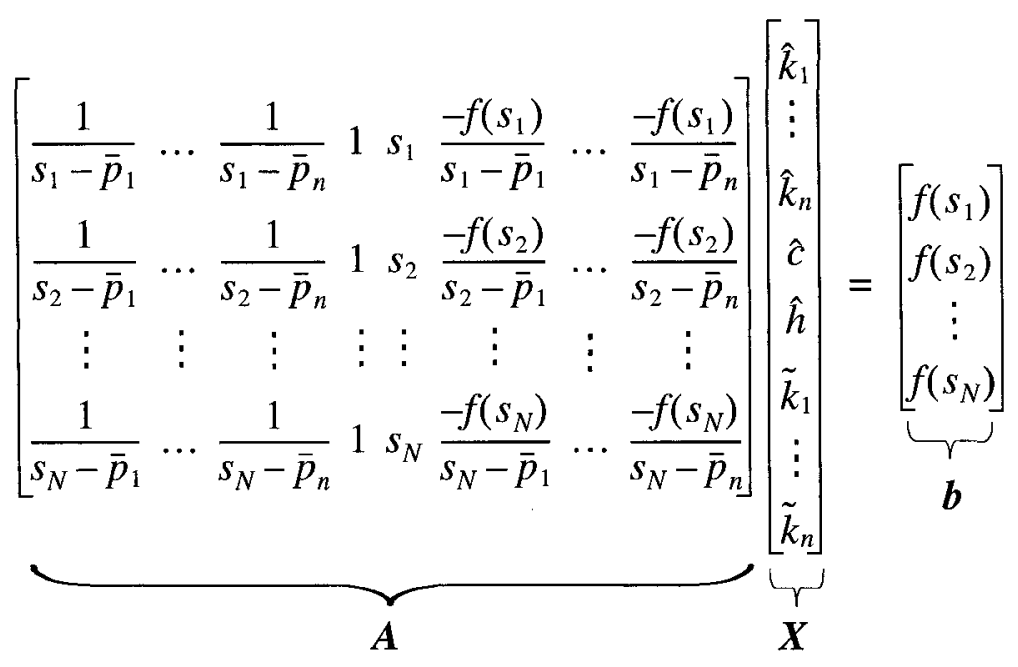

or

$$
\boldsymbol{A} \boldsymbol{X}=\boldsymbol{b}
$$

Notice that for real poles, the unknowns $\boldsymbol{X}=\left[\begin{array}{llllllll}\hat{k}_{1} & \ldots & \hat{k}_{n} & \hat{c} & \hat{h} & \tilde{k}_{1} & \ldots & \tilde{k}_{n}\end{array}\right]^{T}$ are real.

\section{Case 2: Complex Poles}

Complex poles exist in pairs, i.e. with their conjugates. As a result, their residues also exist in complex-conjugate pairs. Consider a complex pole along with its conjugate in (2.24)

$$
\bar{p}_{i}=\operatorname{Re}\left(\bar{p}_{i}\right)+j \operatorname{Im}\left(\bar{p}_{i}\right), \quad \bar{p}_{i}^{*}=\operatorname{Re}\left(\bar{p}_{i}\right)-j \operatorname{Im}\left(\bar{p}_{i}\right)
$$

where $*$ is the complex-conjugate operator. The corresponding residues of the scaled function $\hat{f}(s)$ are

$$
\hat{k}_{i}=\operatorname{Re}\left(\hat{k}_{i}\right)+j \operatorname{Im}\left(\hat{k}_{i}\right), \quad \hat{k}_{i}^{*}=\operatorname{Re}\left(\hat{k}_{i}\right)-j \operatorname{Im}\left(\hat{k}_{i}\right)
$$

while the corresponding residues of scaling function $\sigma(s)$ are 


$$
\tilde{k}_{i}=\operatorname{Re}\left(\tilde{k}_{i}\right)+j \operatorname{Im}\left(\tilde{k}_{i}\right), \quad \tilde{k}_{i}^{*}=\operatorname{Re}\left(\tilde{k}_{i}\right)-j \operatorname{Im}\left(\tilde{k}_{i}\right)
$$

For the above pole pair (2.25) becomes

$$
\frac{\hat{k}_{i}}{s-\bar{p}_{i}}+\frac{\hat{k}_{i}^{*}}{s-\bar{p}_{i}{ }^{*}}+c+h s-f(s) \frac{\tilde{k}_{i}}{s-\bar{p}_{i}}-f(s) \frac{\tilde{k}_{i}^{*}}{s-\bar{p}_{i}^{*}}=f(s)
$$

Substituting (2.31) and (2.32) in (2.33) and after certain mathematical manipulations, we get

$$
\begin{aligned}
& \operatorname{Re}\left(\hat{k}_{i}\right)\left(\frac{1}{s-\bar{p}_{i}}+\frac{1}{s-\bar{p}_{i}{ }^{*}}\right)+\operatorname{Im}\left(\hat{k}_{i}\right)\left(\frac{j}{s-\bar{p}_{i}}-\frac{j}{s-\bar{p}_{i}^{*}}\right)+c+h s \\
& -\operatorname{Re}\left(\tilde{k}_{i}\right) f(s)\left(\frac{1}{s-\bar{p}_{i}}+\frac{1}{s-\bar{p}_{i}^{*}}\right)-\operatorname{Im}\left(\tilde{k}_{i}\right) f(s)\left(\frac{j}{s-\bar{p}_{i}}-\frac{j}{s-\bar{p}_{i}^{*}}\right)=f(s)
\end{aligned}
$$

or

$$
\left[\left(\frac{1}{s-\bar{p}_{i}}+\frac{1}{s-\bar{p}_{i}{ }^{*}}\right)\left(\frac{j}{s-\bar{p}_{i}}-\frac{j}{s-\bar{p}_{i}{ }^{*}}\right) 1 s-f(s)\left(\frac{1}{s-\bar{p}_{i}}+\frac{1}{s-\bar{p}_{i}{ }^{*}}\right)-f(s)\left(\frac{j}{s-\bar{p}_{i}}-\frac{j}{s-\bar{p}_{i}{ }^{*}}\right)\right]\left[\begin{array}{c}
\operatorname{Re}\left(\hat{k}_{i}\right) \\
\operatorname{Im}\left(\hat{k}_{i}\right) \\
c \\
h \\
\operatorname{Re}\left(\tilde{k}_{i}\right) \\
\operatorname{Im}\left(\tilde{k}_{i}\right)
\end{array}\right]=f(s)
$$

Equation (2.35) can be written at several frequencies for $n$ complex poles resulting in the linear matrix equation $\boldsymbol{A} \boldsymbol{X}=\boldsymbol{b}$, where 


$$
\begin{aligned}
& \boldsymbol{A}=\left[\begin{array}{llllll}
\boldsymbol{A}_{1} & \boldsymbol{A}_{2} \ldots \boldsymbol{A}_{i} \ldots \boldsymbol{A}_{n} & \boldsymbol{I} & \boldsymbol{S}
\end{array}\right] ; \quad \boldsymbol{I}=\left[\begin{array}{c}
1 \\
1 \\
\vdots \\
1
\end{array}\right]_{N \times 1} \quad ; \quad \boldsymbol{S}=\left[\begin{array}{c}
s_{1} \\
s_{2} \\
\vdots \\
s_{N}
\end{array}\right] \\
& \boldsymbol{A}_{i}=\left[\begin{array}{cccc}
\left(\frac{1}{s_{1}-\bar{p}_{i}}+\frac{1}{s_{1}-\bar{p}_{i}^{*}}\right) & \left(\frac{j}{s_{1}-\bar{p}_{i}}-\frac{j}{s_{1}-\bar{p}_{i}^{*}}\right) & -f\left(s_{1}\right)\left(\frac{1}{s_{1}-\bar{p}_{i}}+\frac{1}{s_{1}-\bar{p}_{i}^{*}}\right) & -f\left(s_{1}\right)\left(\frac{j}{s_{1}-\bar{p}_{i}}-\frac{j}{s_{1}-\bar{p}_{i}^{*}}\right) \\
\left(\frac{1}{s_{2}-\bar{p}_{i}}+\frac{1}{s_{2}-\bar{p}_{i}^{*}}\right) & \left(\frac{j}{s_{2}-\bar{p}_{i}}-\frac{j}{s_{2}-\bar{p}_{i}^{*}}\right) & -f\left(s_{2}\right)\left(\frac{1}{s_{2}-\bar{p}_{i}}+\frac{1}{s_{2}-\bar{p}_{i}^{*}}\right) & -f\left(s_{2}\right)\left(\frac{j}{s_{2}-\bar{p}_{i}}-\frac{j}{s_{2}-\bar{p}_{i}^{*}}\right) \\
\vdots & \vdots & \vdots \\
\left(\frac{1}{s_{N}-\bar{p}_{i}}+\frac{1}{s_{N}-\bar{p}_{i}^{*}}\right) & \left(\frac{j}{s_{N}-\bar{p}_{i}}-\frac{j}{s_{N}-\bar{p}_{i}^{*}}\right) & -f\left(s_{N}\right)\left(\frac{1}{s_{N}-\bar{p}_{i}}+\frac{1}{s_{N}-\bar{p}_{i}^{*}}\right) & -f\left(s_{N}\right)\left(\frac{j}{s_{N}-\bar{p}_{i}}-\frac{j}{s_{N}-\bar{p}_{i}^{*}}\right)
\end{array}\right] ;
\end{aligned}
$$

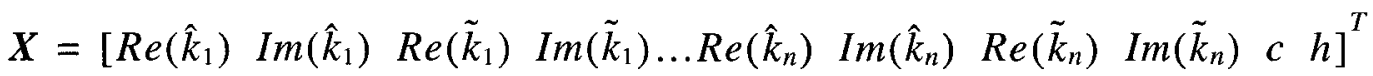

For both the cases, linear formulation $\boldsymbol{A X}=\boldsymbol{b}$ is separated into real and imaginary parts, and subsequently, the following augmented problem is solved

$$
\underbrace{\left[\begin{array}{l}
\operatorname{Re}(\boldsymbol{A}) \\
\operatorname{Im}(\boldsymbol{A})
\end{array}\right]}_{\hat{\boldsymbol{A}}} \boldsymbol{X}=\underbrace{\left[\begin{array}{l}
\operatorname{Re}(\boldsymbol{b}) \\
\operatorname{Im}(\boldsymbol{b})
\end{array}\right]}_{\hat{\boldsymbol{b}}}
$$

or

$$
\hat{A} \boldsymbol{X}=\hat{b}
$$

The over-determined problem of (2.38) is solved by least-square technique or $Q R$ factorization. In the case of least-square method, pre-multiplying both sides of (2.38) by $\hat{A}^{T}$, we get,

$$
\hat{\boldsymbol{A}}^{T} \hat{\boldsymbol{A}} \boldsymbol{X}=\hat{\boldsymbol{A}}^{T} \hat{\boldsymbol{b}}
$$

or 


$$
\boldsymbol{X}=\left(\hat{\boldsymbol{A}}^{T} \hat{\boldsymbol{A}}\right)^{-1} \hat{\boldsymbol{A}}^{T} \hat{\boldsymbol{b}}
$$

While, in the case of using $Q R$ factorization, writing $\hat{A}$ in terms of $Q R$ factors,

$$
\hat{A}=Q R
$$

where $\boldsymbol{Q}$ is an orthogonal matrix, i.e. $\boldsymbol{Q}^{T} \boldsymbol{Q}=\boldsymbol{I}$, and $\boldsymbol{R}$ is an upper triangular matrix. Substituting (2.41) into (2.38) and multiplying both sides by $\boldsymbol{Q}^{T}$, we get

$$
\boldsymbol{R} \boldsymbol{X}=\boldsymbol{Q}^{T} \hat{\boldsymbol{b}}
$$

or

$$
X=\boldsymbol{R}^{-1} \boldsymbol{Q}^{T} \hat{b}
$$

Once the unknowns $\hat{k}_{1} \ldots \hat{k}_{n}, \hat{c}, \hat{h}, \tilde{k}_{1} \ldots \tilde{k}_{n}$ are known (by solving (2.40) or (2.43)), a refined set of poles for $f(s)$ (compared to the initial guess of poles) can be evaluated as described in the following section.

\subsection{2b Refinement of Poles of $f(s)$}

There is a direct equivalence between the poles of $f(s)$ and the zeros of the scaling function $\sigma(s)$, and it is exploited in refining the poles of $f(s)$. For the purpose of illustration, consider the scaling and scaled functions in (2.22) and (2.23) respectively, expressed in the pole-zero form as 


$$
\sigma(s)=\frac{\prod_{i=1}^{n}\left(s-z_{i}\right)}{\prod_{i=1}^{n}\left(s-\bar{p}_{i}\right)} ; \quad \hat{f}(s)=h \frac{\prod_{i=1}^{n+1}\left(s-z_{i}\right)}{n}
$$

From (2.23) we have

$$
f(s) \approx \frac{\hat{f}(s)}{\sigma(s)}
$$

or, substituting (2.44) in (2.45), $f(s)$ can be represented as

$$
f(s)=\frac{\hat{f}(s)}{\sigma(s)}=\frac{\left(\begin{array}{c}
h+1 \\
\left.\prod_{i=1}^{n}\left(s-z_{i}\right)\right) /\left(\prod_{i=1}^{n}\left(s-\bar{p}_{i}\right)\right)
\end{array}\right.}{\left(\prod_{i=1}^{n}\left(s-\tilde{z}_{i}\right)\right) /\left(\prod_{i=1}^{n}\left(s-\bar{p}_{i}\right)\right)}=\frac{\prod_{i=1}^{n+1}\left(s-z_{i}\right)}{\prod_{i=1}^{n}\left(s-z_{i}\right)}
$$

From (2.46) and (2.44), it is clear that the computed zeros of $\sigma(s)$ are in fact the refined poles of $f(s)$ (note that the initial poles cancel in the division process as we started with the same initial poles for $\sigma(s)$ and $\hat{f}(s))$. Thus, calculating the zeros of $\sigma(s)$ leads to a better set of poles than the initial set of poles $p_{i}$, for fitting the original function $f(s)$. Next, a procedure for calculating the zeros of $\sigma(s)$ is described in the following section. 


\subsection{2c Evaluation of Zeros of $\sigma(s)$ (i.e. poles of $f(s)$ )}

Once the unknowns $\hat{k}_{1} \ldots \hat{k}_{n}, \hat{c}, \hat{h}, \tilde{k}_{1} \ldots \tilde{k}_{n}$ are known (by solving (2.40) or (2.43)), zeros of $\sigma(s)$ can be calculated, depending on whether the initial guess for the poles is real or complex, as follows.

\section{Case 1: Real Poles}

It is to be noted that zeros of $\sigma(s)$ correspond to the eigenvalues of the following matrix [26]

$$
\boldsymbol{H}=\Psi-\boldsymbol{g} \phi^{T}
$$

where

$$
\Psi=\left[\begin{array}{cccc}
\bar{p}_{1} & & & \\
& \bar{p}_{2} & & \\
& & \ddots & \\
& & & \bar{p}_{n}
\end{array}\right] ; \quad \boldsymbol{g}=\left[\begin{array}{c}
1 \\
1 \\
\vdots \\
1
\end{array}\right]_{n \times 1} \quad ; \quad \phi^{T}=\left[\begin{array}{llll}
\tilde{k}_{1} & \tilde{k}_{2} & \ldots & \tilde{k}_{n}
\end{array}\right]_{1 \times n}
$$

Notice that $\Psi$ is the diagonal matrix of starting (initial guess) poles, $g$ is a unity column vector. $\phi$ is the column vector of residues of $\sigma(s)$ corresponding to the starting poles (computed using (2.40) or (2.43)). Hence, the refined poles of $f(s)$ can be simply obtained by evaluating the eigenvalues of matrix $\boldsymbol{H}$ in (2.47).

\section{Case 2: Complex Poles}

Similarly, in this case too, the zeros of $\sigma(s)$ correspond to the eigenvalues of the matrix $\boldsymbol{H}$ of (2.47), with $\Psi, \boldsymbol{g}$ and $\phi$ modified as 


$$
\begin{aligned}
& \Psi=\left[\begin{array}{llll}
\Psi_{1} & & & \\
& \Psi_{2} & & \\
& & \ddots & \\
& & \Psi_{n / 2}
\end{array}\right] ; \quad \quad \Psi_{i}=\left[\begin{array}{cc}
\operatorname{Re}\left(\bar{p}_{i}\right) & \operatorname{Im}\left(\bar{p}_{i}\right) \\
-\operatorname{Im}\left(\bar{p}_{i}\right) & \operatorname{Re}\left(\bar{p}_{i}\right)
\end{array}\right] ; \quad i=1,2, \ldots n / 2 \\
& \phi^{T}=\left[\operatorname{Re}\left(\tilde{k}_{1}\right) \operatorname{Im}\left(\tilde{k}_{1}\right) \operatorname{Re}\left(\tilde{k}_{2}\right) \operatorname{Im}\left(\tilde{k}_{2}\right) \ldots \operatorname{Re}\left(\tilde{k}_{n / 2}\right) \operatorname{Im}\left(\tilde{k}_{n / 2}\right)\right]_{1 \times n} \\
& \boldsymbol{g}=\left[\begin{array}{lllllll}
2 & 0 & 2 & 0 & \ldots & 2 & 0
\end{array}\right]_{1 \times n}^{T}
\end{aligned}
$$

Once the zeros of $\sigma(s)$ are calculated, they are used as the new set of starting poles in the next iteration while solving (2.24). The next iteration provides a new set of zeros for $\sigma(s)$ which are again used in the next iteration as starting poles. The iterative process is continued until the poles converge. It is to be noted that during this iterative process, some of the calculated poles may become unstable, i.e. the real part of some of the poles may be positive. This problem is overcome by inverting the sign of their real parts [26]. Successful application of vector fitting requires judicious selection of initial set of poles. Guidelines for the selection of initial set of poles is described in the next section.

\subsection{2d Selection of Initial Set of Poles}

One of the important factors in using vector fitting is selecting the initial set of poles. In [26], following guidelines have been proposed for choosing the initial set of poles. 


\section{a. Functions with distinct resonance peaks}

The starting poles should be complex conjugate with the imaginary parts $\beta_{1}, \beta_{2}, \ldots \beta_{n}$ linearly distributed over the frequency range of interest $\omega_{\min }-\omega_{\max }$. Each pole pair is selected as follows

$$
p=-\alpha \pm j \beta
$$

with

$$
\alpha=\beta / 100
$$

\section{b. Smooth functions}

Real poles, linearly or logarithmically spaced over the frequency range of interest, should be used for approximating the smooth functions.

\subsection{2e Residue Calculation}

Once the poles are known, calculation of residues $k_{i}$, direct constant $c$ and linear term $h$ is carried out using the same procedure as described in Section 2.3.1a (equations (2.16) - (2.18)).

\subsection{2f Calculation of Common Pole-Set for Multiport Networks}

The pole finding step has been described for a scalar function in Section 2.3.2a. However, interconnect networks generally tend to be multiport. If the pole-sets are calculated individually for each element of the transfer function matrix, it can lead to a large number of redundant poles in the macromodel resulting in inefficient transient simulation [28]. Identifying a common multiport pole-set 
from pole-sets of individual elements of the admittance matrix is generally heuristic and can lead to errors. In order to overcome the above problem and to minimize the number of poles in the matrix-transfer function, the following two propositions are used [24], for finding the common pole-set in this thesis.

1) In general, the pole set corresponding to any individual element of the admittance matrix is a subset of the union of all driving point admittances [67].

2) Generally, in a system with large number of dominant poles, pole sets belonging to different driving point admittances contain mostly identical poles and only a very small percentage of poles differ among these sets.

Using the above two propositions, a common pole-set for the multiport poleresidue model is obtained by performing the pole identification algorithm described in Section 2.3.2a on driving point admittances [28]. In addition, the initial set of poles when approximating the vector of driving point admittances, is calculated by running the pole finding iteration on one of the driving point function. This results in faster convergence of pole finding iterations on the vector of driving point functions.

It is evident that the vector fitting s pole-finding iterations are better conditioned compared to the standard least-square approximation because the underlying matrix (2.36) contains terms with $s$ raised to power of one.

\subsubsection{Rational Function Approximation via Nonlinear Formulation}

This method [37] begins by approximating the tabulated function $Y(s)$ by the rational function of the form of (2.7) as follows 


$$
\hat{Y}(\boldsymbol{a}, \boldsymbol{b}, s)=\left.\frac{a_{0}+a_{1} s+a_{2} s^{2}+a_{3} s^{3}+\ldots+a_{m-1} s^{m-1}+a_{m} s^{m}}{1+b_{1} s+b_{2} s^{2}+b_{3} s^{3}+\ldots+b_{n-1} s^{n-1}+b_{n} s^{n}}\right|_{s=s_{k} ; \quad\{k=1,2, \ldots, N\}}
$$

or

$$
\hat{Y}(\boldsymbol{a}, \boldsymbol{b}, \boldsymbol{s})=\frac{\sum_{i=0}^{m} a_{i} s^{i}}{\sum_{i=0}^{n} b_{i} s^{i}}
$$

where $\boldsymbol{b}=\left[\begin{array}{llll}b_{0} & b_{1} & \ldots & b_{n}\end{array}\right], b_{0}=1, \boldsymbol{a}=\left[\begin{array}{lll}a_{0} & \ldots & a_{m}\end{array}\right]$.

However, instead of linearising the problem of identifying the unknown coefficients $\boldsymbol{b}=\left[\begin{array}{llll}b_{0} & b_{1} & \ldots & b_{n}\end{array}\right]$ and $\boldsymbol{a}=\left[\begin{array}{lll}a_{0} & \ldots & a_{m}\end{array}\right]$, (2.52) is solved using Guass-Newton type of method with an initial guess for coefficients [37]. The details of this procedure are described below.

Given a sampled transfer function $Y\left(s_{h}\right)$, at $N$ discrete frequency points $h=1,2, \ldots N$, the rational approximation problem amounts to minimizing the error

$$
E\left(s_{h}\right)=\hat{Y}\left(\boldsymbol{a}, \boldsymbol{b}, s_{h}\right)-Y\left(s_{h}\right) ; \quad(h=1,2, \ldots N)
$$

in some appropriate norm.

When minimizing 2-norm, problem in (2.54) can be expressed as [37]

$$
\|E(\boldsymbol{a}, \boldsymbol{b}, s)\|_{2}=\sqrt{\sum_{h=1}^{N}\left(\left\|\hat{Y}\left(\boldsymbol{a}, \boldsymbol{b}, s_{h}\right)-Y\left(s_{h}\right)\right\|_{2}\right)^{2}}
$$


In turn (2.55) is equivalent to minimizing

$$
f(x)=\left(\|E(\boldsymbol{a}, \boldsymbol{b}, s)\|_{2}\right)^{2}=\hat{\boldsymbol{E}}(\boldsymbol{a}, \boldsymbol{b}, s)^{T} \hat{\boldsymbol{E}}(\boldsymbol{a}, \boldsymbol{b}, s)
$$

with

$$
\hat{\boldsymbol{E}}(\boldsymbol{a}, \boldsymbol{b}, s)^{T}=\left[\begin{array}{llllll}
\operatorname{Re}\left(E\left(s_{1}\right)\right) & \ldots & \operatorname{Re}\left(E\left(s_{N}\right)\right) & \operatorname{Im}\left(E\left(s_{1}\right)\right) & \ldots & \operatorname{Im}\left(E\left(s_{N}\right)\right)
\end{array}\right]
$$

and

$$
\boldsymbol{x}=\left[\begin{array}{ll}
a & b
\end{array}\right]
$$

The problem in (2.56) is solved by Levenberg-Marquardt method [37], which uses a linearized model $\hat{\boldsymbol{M}}$ of $\hat{\boldsymbol{E}}(\boldsymbol{x})$ around $\boldsymbol{x}_{c}$ (based on Newton-Raphson iterations)

$$
\hat{\boldsymbol{M}}\left(\boldsymbol{x}_{c}\right)=\hat{\boldsymbol{E}}\left(\boldsymbol{x}_{c}\right)+\boldsymbol{J}\left(\boldsymbol{x}_{c}\right)\left(\boldsymbol{x}-\boldsymbol{x}_{c}\right)
$$

where $\boldsymbol{J}\left(\boldsymbol{x}_{c}\right)$ is the Jacobian of $\hat{\boldsymbol{E}}$ at $\boldsymbol{x}_{c}$. The method minimizes, at each step, the model error, subject to the following constraint

$$
\begin{gathered}
\min \left\|\hat{\boldsymbol{E}}\left(\boldsymbol{x}_{c}\right)+J\left(\boldsymbol{x}_{c}\right) \delta \boldsymbol{x}\right\|_{2} ; \text { such that } \\
\delta \boldsymbol{x} \in \mathfrak{R}^{m+n+1} \\
\|\delta \boldsymbol{x}\|_{2} \leq \delta_{c}
\end{gathered}
$$

where $\delta_{c}$ is the desired tolerance on $\|\delta\|_{2}$. The problem in (2.60) is solved by Gauss-Newton s method. However, the Jacobian $\boldsymbol{J}\left(\boldsymbol{x}_{c}\right)$ loses numerical rank rapidly, as the order increases, and therefore the solution of (2.60) becomes illconditioned. This phenomenon can be understood by noting from (2.55) that the Jacobian $\boldsymbol{J}\left(\boldsymbol{x}_{k-1}\right)$ is simply the Jacobian of 


$$
\left[\operatorname{Re}\left\{\hat{Y}_{k-1}\left(s_{1}\right)\right\} \ldots \operatorname{Re}\left\{\hat{Y}_{k-1}\left(s_{N}\right)\right\} \operatorname{Im}\left\{\hat{Y}_{k-1}\left(s_{1}\right)\right\} \ldots \operatorname{Im}\left\{\hat{Y}_{k-1}\left(s_{N}\right)\right\}\right]^{T}
$$

Consequently (2.59) can be written as

$$
\underbrace{-\left[\begin{array}{lll}
\operatorname{Re}\left\{\boldsymbol{D}_{k-1}^{-1} \boldsymbol{U}_{m}\right\} & \ldots \operatorname{Re}\left\{-\boldsymbol{D}_{k-1}^{-1} \hat{\boldsymbol{Y}}_{k-1} \boldsymbol{U}_{n}\right\} \\
\operatorname{Im}\left\{\boldsymbol{D}_{k-1}^{-1} \boldsymbol{U}_{m}\right\} & \ldots \operatorname{Im}\left\{-\boldsymbol{D}_{k-1}^{-1} \hat{\boldsymbol{Y}}_{k-1} \boldsymbol{U}_{n}\right\}
\end{array}\right]}_{\boldsymbol{J}\left(\boldsymbol{x}_{k-1}\right)}\left[\begin{array}{l}
\Delta \boldsymbol{a} \\
\Delta \boldsymbol{b}
\end{array}\right]=\left[\begin{array}{c}
\operatorname{Re}\left\{\hat{Y}_{k-1}\left(s_{1}\right)-Y\left(s_{1}\right)\right\} \\
\vdots \\
\operatorname{Re}\left\{\hat{Y}_{k-1}\left(s_{N}\right)-Y\left(s_{N}\right)\right\} \\
\operatorname{Im}\left\{\hat{Y}_{k-1}\left(s_{1}\right)-Y\left(s_{1}\right)\right\} \\
\vdots \\
\operatorname{Im}\left\{\hat{Y}_{k-1}\left(s_{N}\right)-Y\left(s_{N}\right)\right\}
\end{array}\right]
$$

where, $\boldsymbol{U}_{m}$ is a Vandermonde matrix and $\Delta \boldsymbol{a}, \Delta \boldsymbol{b}$ are the updates of $\boldsymbol{a}$ and $\boldsymbol{b}$ respectively.

$$
\boldsymbol{U}_{m}=\left[\begin{array}{ccccc}
1 & s_{1} & s_{1}^{2} & \ldots & s_{1}^{m} \\
1 & s_{2} & s_{2}^{2} & \ldots & s_{2}^{m} \\
\vdots & \vdots & \vdots & \ddots & \vdots \\
1 & s_{N} & s_{N}^{2} & \ldots & s_{N}^{m}
\end{array}\right],
$$

$\boldsymbol{D}_{k-1}(s)$ is a diagonal matrix of the denominator of $\hat{Y}(s)$ at iteration number $k-1$, shown below 


$$
\boldsymbol{D}_{k-1}(s)=\left[\begin{array}{cccc}
\sum_{i=0}^{n} b_{i} s_{1}{ }^{i} & 0 & \ldots & 0 \\
0 & \sum_{i=0}^{n} b_{i} s_{2}{ }^{i} & 0 & 0 \\
\vdots & \vdots & \ddots & \vdots \\
0 & 0 & 0 & \sum_{i=0}^{n} b_{i} s_{N}
\end{array}\right],
$$

$\hat{\boldsymbol{Y}}_{k-1}(s)$ is the diagonal matrix of approximation of $\hat{Y}(s)$ at iteration number $k-1$

$$
\hat{\boldsymbol{Y}}_{k-1}(s)=\left[\begin{array}{cccc}
\hat{Y}_{k-1}\left(s_{1}\right) & 0 & \ldots & 0 \\
0 & \hat{Y}_{k-1}\left(s_{2}\right) & 0 & 0 \\
\vdots & \vdots & \ddots & \vdots \\
0 & 0 & 0 & \hat{Y}_{k-1}\left(s_{N}\right)
\end{array}\right]
$$

It can be easily seen that the Vandermonde matrix of (2.63) becomes highly illconditioned as the order of approximation increases, thus rendering the Jacobian $\boldsymbol{J}\left(\boldsymbol{x}_{k-1}\right)$ also ill-conditioned. This is likely to stall the Gauss-Newton procedure in (2.62). To deal with this ill-conditioning problem, it has been proposed in [37] to generate an orthonormal polynomial basis that spans the same space as the columns of Vandermonde matrix of (2.63). From (2.63) it is evident that $\boldsymbol{U}_{m}$ can be expressed as

$$
\left[\begin{array}{lllll}
I & S 1 & S^{2} I & \ldots & S^{m} \boldsymbol{I}
\end{array}\right] ; \boldsymbol{I}=\left[\begin{array}{c}
1 \\
1 \\
\vdots \\
1
\end{array}\right]_{N \times 1}
$$


where,

$$
\boldsymbol{S}=\left[\begin{array}{cccc}
s_{1} & 0 & \ldots & 0 \\
0 & s_{2} & 0 & 0 \\
\vdots & \vdots & \ddots & \vdots \\
0 & 0 & 0 & s_{N}
\end{array}\right]
$$

An orthonormal basis for $\boldsymbol{U}_{m}$ can be obtained using an Arnoldi Process [68] as follows

$$
S \hat{V}=\hat{V} \hat{H}
$$

where, $\hat{\boldsymbol{V}} \in \mathfrak{R}^{N \times(m+1)}$ is an orthogonal matrix, i.e. $\hat{\boldsymbol{V}}^{T} \hat{\boldsymbol{V}}=\boldsymbol{I}$ and $\hat{\boldsymbol{H}} \in \mathfrak{R}^{(m+1) \times(m+1)}$ is an upper Hessenberg matrix [68]. The column space of $\hat{\boldsymbol{V}}$ spans the same space as that of the columns of $\boldsymbol{U}_{m}$. Notice that since $\boldsymbol{S}$ is Skew-Hermitian so is $\hat{\boldsymbol{H}}$. But $\hat{\boldsymbol{H}}$ is also upper Hessenberg by definition. Therefore it has to be a tridiagonal matrix. In this case, the Arnoldi process becomes a Lanczos process. In other words this is a process of constructing a polynomial basis defined by the three term recursion

$$
\sum_{i=1}^{j} h_{i, j} P_{i-1}(s)+h_{j+1, j} P_{j}(s)=s P_{j-1}(s)
$$

where, $P_{j}(s)$ is a polynomial of order $j,(j=1$ to $m)$ and $h_{i, j}$ are the elements of $\hat{\boldsymbol{H}}$. The polynomial described above satisfies the discrete orthogonality relation

$$
\sum_{h=1}^{N} P_{j}\left(s_{h}\right) P_{i}\left(s_{h}\right)=0
$$

Replacing Vandermonde matrices in (2.62) by the new basis matrices we get 


$$
-\left[\begin{array}{llll}
\operatorname{Re}\left\{\boldsymbol{D}_{k-1}^{-1} \hat{\boldsymbol{V}}_{m}\right\} & \ldots & \operatorname{Re}\left\{-\boldsymbol{D}_{k-1}^{-1} \hat{\boldsymbol{Y}}_{k-1} \hat{\boldsymbol{V}}_{n}\right\} \\
\operatorname{Im}\left\{\boldsymbol{D}_{k-1}^{-1} \hat{\boldsymbol{V}}_{m}\right\} & \ldots & \operatorname{Im}\left\{-\boldsymbol{D}_{k-1}^{-1} \hat{\boldsymbol{Y}}_{k-1} \hat{\boldsymbol{V}}_{n}\right\}
\end{array}\right]\left[\begin{array}{l}
\Delta \tilde{\boldsymbol{a}} \\
\Delta \tilde{b}
\end{array}\right]=\left[\begin{array}{c}
\operatorname{Re}\left\{\hat{\boldsymbol{Y}}_{k-1}\left(s_{1}\right)-Y\left(s_{1}\right)\right\} \\
\vdots \\
\operatorname{Re}\left\{\hat{Y}_{k-1}\left(s_{N}\right)-Y\left(s_{N}\right)\right\} \\
\operatorname{Im}\left\{\hat{\boldsymbol{Y}}_{k-1}\left(s_{1}\right)-Y\left(s_{1}\right)\right\} \\
\vdots \\
\operatorname{Im}\left\{\hat{Y}_{k-1}\left(s_{N}\right)-Y\left(s_{N}\right)\right\}
\end{array}\right]
$$

which can be solved without the problem of ill-conditioning associated with the higher order of approximations.

By changing from the monomial basis to the new polynomial basis, (2.53) can be written as

$$
\hat{Y}(\tilde{\boldsymbol{a}}, \tilde{b}, s)=\frac{\sum_{i=0}^{m} \tilde{a}_{i} P_{i}(s)}{\sum_{i=0}^{n} \tilde{b}_{i} P_{i}(s)}
$$

where $P_{i}(s)$ is described in (2.69).

Since the method described above is a non-linear problem, a good solution of (2.71) depends on the starting guess of the coefficients $\tilde{a}_{i}{ }^{\prime} s$ and $\tilde{b}_{i}{ }^{\prime} s$. A good initial guess for these coefficients can be obtained by multiplying (2.72) by its denominator on both sides and solving the overdetermined linear least-square problem [37]. 


\subsection{Macromodeling Issues: Passivity}

The rational function based algorithms of Section 2.3.1 - Section 2.3.3 dealt mainly with the accuracy aspect, when approximating the tabulated data. These methods can produce accurate, stable rational function approximations, however, they may fail to guarantee the important physical property of passivity, of the resulting approximation. It was during the period of development of these algorithms, that the importance of the passivity of the reduced-order model was being recognised in the area of model-reduction. It was realised that, since the interconnect networks are passive by nature, it is necessary that the reduced-order models representing their behaviour should also be passive. This is because, stable but non-passive models may lead to unstable systems when connected to other passive components [5], [10], [11], [13] leading to oscillatory transient responses. For the purpose of illustration, the transient response of a stable but non-passive macromodel, when connected to an external linear resistive load [24] is given in Fig. 2.3. On the other hand, a passive macromodel, when terminated with any arbitrary passive load always guarantees the stability of the overall resulting network.

In view of the above, research fraternity in the interconnect area started focussing on the issue of the passivity of the macromodels of tabulated data. In the subsequent publications, the issue of passivity was gradually being addressed, from an initial adhoc treatment to more advanced methodologies later on. In the next section, a review of the algorithms for ensuring the passivity of the macromodels of tabulated networks is described. 


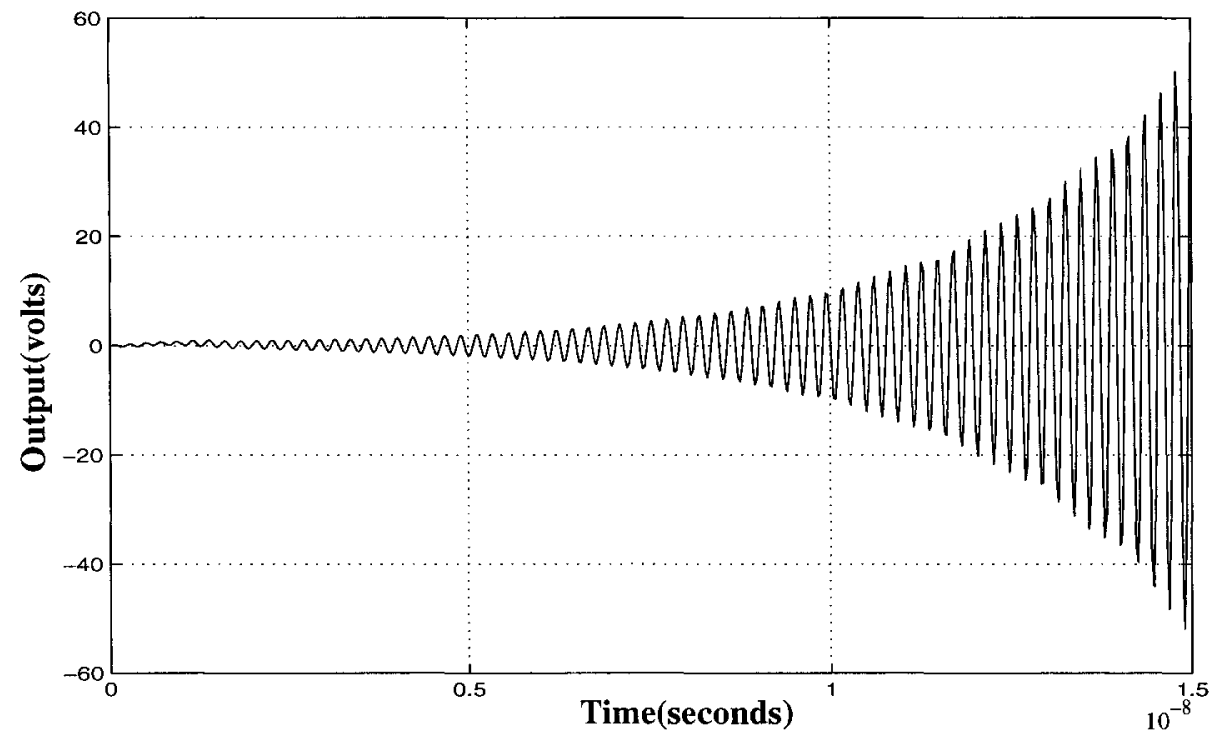

Fig. 2.3. Transient response of a stable but non-passive macromodel with passive terminations

\subsection{Review of Passive Macromodeling Algorithms for Tabulated Data}

Some of the existing algorithms which guarantee passivity of the rational approximation model of the tabulated data of interconnect networks, are presented in this section.

\subsubsection{Passive Rational Function Approximation Using Nevanlinna-Pick Interpolation (NPI)}

This algorithm is based on Nevanlinna-Pick interpolation [32], [33]. Given the sampled scattering matrix of a passive system, the method finds a guaranteed bounded-real matrix rational function approximation, while at the same time 
matching the frequency response of the original network. For the case of scattering parameters, a system is passive if its scattering matrix is bounded-real. A matrix $S(s)$, with each element described by rational function, is bounded real if the following conditions are satisfied [69]:

(i) $S^{*}(s)=S\left(s^{*}\right)$

(ii) $S(s)$ is analytic in $\operatorname{Re}(s)>0$

(iii) $\|\boldsymbol{S}(j \omega)\|_{2} \leq 1$ for $\omega \in \Re$

The problem formulation of the NPI method for the frequency sampled scattering parameters is described below.

Let $\Delta$ represent the open right-half plane. Given a set of scattering matrices $S\left(s_{h}\right) \in \zeta^{m \times m}(h=1,2 \ldots N)$ at $N$ frequency points, $m$ is the number of input/ output ports, $\zeta$ represents the complex domain. The $m \times m$ matrix rational function $\boldsymbol{F}(s)$ interpolates the data set $\left(s_{h}, S\left(s_{h}\right)\right)$ with $h=1,2 \ldots N$, if

$$
\boldsymbol{F}\left(s_{h}\right)=\boldsymbol{S}\left(s_{h}\right), \quad h=1,2, \ldots N
$$

The Nevanlinna-Pick problem is to find an interpolating function $\boldsymbol{F}(s)$, that is analytic in $\Delta$, interpolates the data set $\left(s_{h}, S\left(s_{h}\right)\right)$ as in (2.73), and satisfies

$$
\|\boldsymbol{F}(s)\|_{2}<1 ; s \in \Delta
$$

Now, let

$$
\boldsymbol{C}_{-}=\left[\begin{array}{lll}
\boldsymbol{I}_{m} & \ldots & \boldsymbol{I}_{m}
\end{array}\right],
$$


a $m \times N m$ matrix, $\left(\boldsymbol{I}_{m}\right.$ is a $m \times m$ identity matrix)

$$
C_{+}=\left[\begin{array}{lll}
S_{1} & \ldots & S_{N}
\end{array}\right]
$$

a $m \times N m$ matrix, (here $S_{i}$ stands for $S\left(s_{i}\right)$ ) and

$$
\boldsymbol{A}_{\pi}=\left[\begin{array}{ccc}
\boldsymbol{s}_{1} \boldsymbol{I}_{m} & & \boldsymbol{0} \\
& \ddots & \\
\boldsymbol{0} & & \boldsymbol{s}_{N} \boldsymbol{I}_{m}
\end{array}\right],
$$

a $N m \times N m$ block diagonal matrix

Theorem (Matrix Nevanlinna-Pick): There exists a matrix rational function $\boldsymbol{F}(s)$ that interpolates the given data set $(s, S)$, that is analytic in $\Delta$ and satisfies $\|\boldsymbol{F}(s)\|_{2}<1 ; s \in \Delta$, if and only if the Pick matrix,

$$
\boldsymbol{\Lambda}_{p, q}=\left[\frac{\boldsymbol{I}-\boldsymbol{S}_{p}^{H} \boldsymbol{S}_{q}}{\boldsymbol{S}_{p}^{*}+\boldsymbol{s}_{q}}\right]_{1 \leq p, q \leq N}
$$

is positive definite (the operator $H$ indicates the hermitian operator). In this case, there is a $2 \times 2$ block matrix function

$$
\Theta(s)=\boldsymbol{I}_{2 m}+\left[\begin{array}{l}
\boldsymbol{C}_{+} \\
\boldsymbol{C}_{-}
\end{array}\right]\left(s \boldsymbol{I}_{N m}-\boldsymbol{A}_{\pi}\right)^{-1} \boldsymbol{\Lambda}^{-1}\left[\begin{array}{ll}
\boldsymbol{C}_{+}^{H} & \boldsymbol{C}_{-}^{H}
\end{array}\right]=\left[\begin{array}{ll}
\Theta_{1,1}(s) & \Theta_{1,2}(s) \\
\Theta_{2,1}(s) & \Theta_{2,2}(s)
\end{array}\right]
$$

such that the solution of the Nevanlinna-Pick interpolation becomes [33]

$$
\boldsymbol{F}(s)=\left[\Theta_{1,1}(s) \boldsymbol{G}(s)+\Theta_{1,2}(s)\right]\left[\Theta_{2,1}(s) \boldsymbol{G}(s)+\Theta_{2,2}(s)\right]^{-1}
$$


where $\boldsymbol{G}(s)$ is an arbitrary $m \times m$ rational matrix function that is analytic in $\Delta$ and satisfies $\|\boldsymbol{G}(s)\|_{2}<1, \quad s \in \Delta$.

In the above theorem, the data points are assumed to be in the interior of the $\Delta$, i.e. the open right-half plane, but the frequency sampled data is essentially available on the imaginary axis. The identification then becomes the boundary interpolation problem, also called a Loewner interpolation problem [33], in which (2.78) - (2.80) are evaluated by applying a small shift $\sigma$ to $s$.

At first glance, this approach may seem to be straightforward, but there are several critical issues, which limit the practicality of the method. They are as follows.

- From (2.80), the Nevanlinna-Pick interpolant will have at least $N m$ poles (e.g. a 3-port system with 200 data points will have 600 poles). A systematic approach is required to reduce the number of poles.

- The choice of $G(s)$ is critical to the accuracy of the fitting process. The appropriate choice of $\boldsymbol{G}(s)$ often leads to the non-linear optimization problem [33], which can be expensive and may not always converge.

- The choice of $\sigma$ is critical to the positive definiteness of the pick matrix in (2.78). On one hand, too small a value can lead to pure imaginary poles, leading to oscillatory response, while on the other hand too large a value may lead to a pick matrix of (2.78) not being positive definite. 


\subsubsection{Convex Programming Approach to Positive-Real Rational Approximation}

A convex programming approach to the positive-real approximation of tabulated data has been recently suggested in [34], [35]. A brief review of this algorithm is provided below (from the point of view of the admittance/impedance parameters).

The tabulated frequency data of a MIMO (multiple input, multiple output) system is approximated by rational functions as described in Section 2.3 and converted to the state-space form

$$
\begin{aligned}
\dot{\boldsymbol{x}}(t) & =\boldsymbol{A} \boldsymbol{x}(t)+\boldsymbol{B} u(t) \\
y(t) & =\boldsymbol{C} \boldsymbol{x}(t)+\boldsymbol{D} u(t)
\end{aligned}
$$

The transfer function corresponding to (2.81) can be written as

$$
\boldsymbol{H}(s)=\boldsymbol{C}(s \boldsymbol{I}-\boldsymbol{A})^{-1} \boldsymbol{B}+\boldsymbol{D}
$$

The poles of the system constitute the state matrix $\boldsymbol{A} \in \mathfrak{R}^{n \times n} . \quad \boldsymbol{B} \in \mathfrak{R}^{n \times m}$ represents the input mapping matrix. $C \in \mathfrak{R}^{m \times n}$ represents the output mapping matrix and contains the residues of the system. $\boldsymbol{D} \in \mathfrak{R}^{m \times m}$ consists of the direct coupling terms. $n$ represents the order of approximation, while $m$ represents the number of input/output ports. The passivity of the system represented by (2.81) and positive realness of the transfer function $\boldsymbol{H}(s)$ is related by the PositiveReal Lemma [70]. 
Positive-Real Lemma: The state-space system $(\boldsymbol{A}, \boldsymbol{B}, \boldsymbol{C}, \boldsymbol{D})$ is passive (or in other words the transfer function $\boldsymbol{H}(s)$ is positive-real) if and only if the following LMIs are satisfied [70]

$$
\begin{aligned}
{\left[\begin{array}{cc}
-\boldsymbol{A}^{T} \boldsymbol{P}-\boldsymbol{P A} & -\boldsymbol{P B}+\boldsymbol{C}^{T} \\
-\boldsymbol{B}^{T} \boldsymbol{P}+\boldsymbol{C} & \boldsymbol{D}+\boldsymbol{D}^{T}
\end{array}\right] } & \geq \mathbf{0} \\
\boldsymbol{P} & \geq \mathbf{0}
\end{aligned}
$$

The algorithm starts by assuming that a suitable rational function approximation of the tabulated data with desired accuracy has been obtained. This rational function approximation however, may be nonpassive. The idea here is to keep the poles (i.e. matrix $\boldsymbol{A}$ ) constant and perturb the residues (i.e. matrix $\boldsymbol{C}$ ) and direct coupling constants to obtain a passive macromodel which satisfies a given error criteria for approximation and the LMIs in (2.83). This is achieved by formulating the optimization problem as described below.

\subsection{2a Formulation of the optimization problem}

Let the tabulated data for the admittance matrix represented by $\tilde{\boldsymbol{H}}\left(s_{h}\right)$ be given for $N$ frequency points $s_{1}$ through $s_{N}$. The transfer function approximating this data is as defined by $\boldsymbol{H}(s)$ in (2.82). Noting that $\boldsymbol{H}_{p, q}(s)$ and $\tilde{\boldsymbol{H}}_{p, q}(s)$ represent the $(p, q)$ entry of the transfer function of the model and the data matrix respectively, the idea is to calculate the $\boldsymbol{C}$ and $\boldsymbol{D}$ such that the new model is passive and at the same time the error in the response of each entry of the transfer matrix is minimized, i.e. the following cost function is minimized for each entry of the transfer function 


$$
\sum_{h=1}^{N}\left\|\boldsymbol{H}_{p, q}\left(s_{h}\right)-\tilde{\boldsymbol{H}}_{p, q}\left(s_{h}\right)\right\|_{2}^{2}<t_{p, q}
$$

The constraint (2.84) can be further compressed as follows. Let $\boldsymbol{B}_{q}$ represents the $q^{t h}$ column of $\boldsymbol{B}$ and $\boldsymbol{C}_{p}$ represents the $p^{t h}$ row of $\boldsymbol{C}$. Let $\boldsymbol{e}_{p}$ represents the $p^{t h}$ column of the identity matrix of size $m \times m$. Next define $\boldsymbol{F}_{p, q} \in \Re^{2 N \times(n+m)}$ and $\boldsymbol{G}_{p, q} \in \mathfrak{R}^{2 N}$ as

$$
\begin{gathered}
\boldsymbol{F}_{p, q}(h,:)=\left\{\begin{array}{cc}
\operatorname{Re}\left(\boldsymbol{J}\left(s_{h}\right)\right) & h \leq N \\
\operatorname{Im}\left(\boldsymbol{J}\left(s_{h-N}\right)\right) & h>N
\end{array}\right. \\
\boldsymbol{G}_{p, q}(h)=\left\{\begin{array}{cc}
\operatorname{Re}\left(\tilde{\boldsymbol{H}}_{p, q}\left(s_{h}\right)\right) & h \leq N \\
\operatorname{Im}\left(\tilde{\boldsymbol{H}}_{p, q}\left(s_{h-N}\right)\right) & h>N
\end{array}\right.
\end{gathered}
$$

where

$$
\boldsymbol{J}(s)=\left[\boldsymbol{B}_{q}^{T}\left(s_{h} \boldsymbol{I}-\boldsymbol{A}^{T}\right)^{-1} \quad \boldsymbol{e}_{q}^{T}\right]
$$

With $\boldsymbol{X}_{p}$ denoting the $p^{\text {th }}$ column of

$$
\boldsymbol{X}=\left[\begin{array}{l}
\boldsymbol{C}^{T} \\
\boldsymbol{D}^{T}
\end{array}\right]
$$

(2.84) can be written as

$$
\sum_{h=1}^{N}\left\|\boldsymbol{H}_{p, q}\left(s_{h}\right)-\tilde{\boldsymbol{H}}_{p, q}\left(s_{h}\right)\right\|_{2}^{2}=\left\|\boldsymbol{F}_{p, q} \boldsymbol{X}_{p}-\boldsymbol{G}_{p, q}\right\|_{2}^{2}
$$

Performing the QR decomposition on $\boldsymbol{F}_{p, q}$ as 


$$
\boldsymbol{F}_{p, q}=\boldsymbol{Q}_{p, q} \boldsymbol{R}_{p, q}
$$

(2.89) can be expressed as

$$
\sum_{h=1}^{N}\left\|\boldsymbol{H}_{p, q}\left(s_{h}\right)-\tilde{\boldsymbol{H}}_{p, q}\left(s_{h}\right)\right\|_{2}^{2}=\boldsymbol{E}_{p, q}^{T} \boldsymbol{E}_{p, q}+\delta_{p, q}^{2}
$$

where

$$
\boldsymbol{E}_{p, q}=\left(\boldsymbol{R}_{p, q} \boldsymbol{X}_{p}-\boldsymbol{Q}_{p, q}^{T} \boldsymbol{G}_{p, q}\right)
$$

and

$$
\delta_{p, q}^{2}=\boldsymbol{G}_{p, q}^{T}\left(\boldsymbol{I}-\boldsymbol{Q}_{p, q} \boldsymbol{Q}_{p, q}^{T}\right) \boldsymbol{G}_{p, q}
$$

With the above definitions the constraint (2.84) becomes

$$
\boldsymbol{E}_{p, q}^{T} \boldsymbol{E}_{p, q}+\delta_{p, q}^{2}<t_{p, q}
$$

The optimization problem for obtaining the passive macromodel can thus be stated as

$$
\begin{gathered}
\text { minimize } t(\boldsymbol{C}, \boldsymbol{P}) \\
\text { such that : }\left[\begin{array}{rr}
-\boldsymbol{A}^{T} \boldsymbol{P}-\boldsymbol{P} \boldsymbol{A} & -\boldsymbol{P} \boldsymbol{B}+\boldsymbol{C}^{T} \\
-\boldsymbol{B}^{T} \boldsymbol{P}+\boldsymbol{C} & \boldsymbol{D}+\boldsymbol{D}^{T}
\end{array}\right] \geq \mathbf{0} \\
\boldsymbol{P} \geq \mathbf{0} \\
\boldsymbol{E}_{p, q}^{T} \boldsymbol{E}_{p, q}+\delta_{p, q}^{2}<t_{p, q} \\
t \geq 0 \\
t_{p, q} \leq t \mid \text { for } \quad 1 \leq p, q \leq m .
\end{gathered}
$$


The objective function and the constraints in (2.95) are convex functions of $\boldsymbol{C}, \boldsymbol{P}$ and $t_{p, q}$ and can be solved using standard semidefinite programming (SDP) techniques [34].

However, with this approach, the computational cost exhibits rapid growth with the order $(n)$ of the state-space system. The cost of forming the matrix needed to compute the step direction during each iteration of the optimization is roughly $O\left(n^{6}\right)$ [34]. Some improvements have been reported in the formulation of the above problem which can bring down this cost to $O\left(m n^{4}\right)$ [34]. However, the total cost of this technique still remains high. One of the reasons for this can be attributed to the fact that this algorithm, while calculating the matrix $\boldsymbol{C}$ conforming to passivity conditions, also generates the redundant matrix $\boldsymbol{P} \in \Re^{n \times n}$. Hence, the resources are unnecessarily used to evaluate the $\boldsymbol{P}$-matrix which is of no use for the desired purpose of obtaining passive model.

It is evident that the rational function based algorithms described in Section 2.3 and Section 2.5 generate the pole-residue approximation for the tabulated data in the form of (2.13), (2.21). Once this frequency-domain model is obtained, it can be linked to the transient simulator along with other nonlinear circuit elements in two ways [48]

1. Synthesis: A macromodel using equivalent circuit elements is formed, which can be analysed by any time-domain circuit simulator, such as SPICE. There are several ways of achieving this. The prominent ones among them can be found in [48]. 
2. Recursive Convolution: The recursive convolution and indirect numerical integration are used for fast transient simulation of pole-residue formulation [19]. The details of this technique is given in Appendix A.

\subsection{Conclusion}

This chapter has discussed some of the prominent techniques for including frequency sampled data in overall circuit simulation. Techniques based on convolution and rational function approximation have been described. Problems such as ill-conditioning and ensuring passivity have been discussed and the algorithms overcoming them are described.

As pointed out in chapter 1 that, a multiport, broadband macromodel of a distributed interconnect network tend to be very large and hence, is computationally time consuming during time-domain simulation. Several modelorder reduction algorithms have been proposed to ease the computational burden by reducing the order of the macromodel. A review of these algorithms is presented in the next chapter. 


\section{CHAPTER 3 Review of Model Order Reduction Techniques}

It was pointed out in chapter 1 that, the models of interconnect networks tend to be very large and their direct simulation is prohibitively CPU intensive. Modelorder reduction (MOR) algorithms have been recently proposed to address this problem. MOR techniques essentially remove the states of the system which have insignificant contribution to the response and can be broadly classified into two categories. The first category of algorithms aim for the first-level reduction of large systems [6] - [8], [10] - [13]. These algorithms are further classified as direct moment-matching [6] - [8] or indirect moment-matching [10] - [13] based approaches. Second category of algorithms aim for second-level reduction. These algorithms are applied on reduced order systems resulting from the first category of algorithms, and are based on balancing and truncation techniques [71] - [74].

The rest of this chapter is organised as follows. Section 3.1 describes the concept of direct moment-matching algorithm. Indirect moment-matching based on Arnoldi procedure and congruent transformation (PRIMA) is discussed in Section 3.2. Truncated balanced realization (TBR) based second-level reduction algorithm is discussed in Section 3.3. 


\subsection{Direct Moment Matching Techniques}

In the context of VLSI, the initial model-reduction techniques were mainly based on the direct moment-matching and Pad approximation [6] - [9]. The crux of moment-matching techniques (MMT) is the extraction of a small set of dominant poles and residues of a large network. This lower-order approximation is obtained using the Taylor series coefficients (moments) of the system and performing Pad approximation using the Laplace-domain transfer function. Such reduced-order models can be used to estimate the transient response of a linear network over a predetermined range of excitation frequencies. Reducedorder frequency-domain models can be easily translated into a set of ordinary differential equations, thus enabling a mixed frequency/time simulation. The prominent reduction techniques based on explicit moment-matching are asymptotic waveform evaluation (AWE) [6], [7] and complex frequency hopping (CFH) [7], [8].

AWE is based on the Taylor series expansion and Pad approximation at $s=0$. Since Pad approximation is accurate only near the point of expansion, reduced-order models obtained through AWE suffer from accuracy. This problem was addressed by the introduction of $\mathrm{CFH}$ [7], [8]. CFH ensures the accuracy of an approximation over a desired frequency range using Pad approximations at multiple expansion points (hops). However, direct moment matching techniques based on Pad approximation suffer from two major problems: (a) ill-conditioning associated with the moment matrix, (b) no guarantee of the passivity of the reduced model. In order to address these difficulties, an alternative stream of algorithms, which can be classified as indirect moment-matching techniques were developed [10] - [13]. They are described in the next section. 


\subsection{Indirect Moment Matching Technique}

Indirect moment-matching algorithms [10] - [14] use the Krylov-subspace techniques and congruent transformation procedure to obtain a reduced order system. Krylov-subspace methods are based on the computation of eigenvalues with largest magnitude. They overcome the problem of ill-conditioning associated with the direct moment matching techniques by utilising the well conditioned algorithms such as Arnoldi process [68]. As a result, Krylov methods can capture hundreds of poles at a single expansion point compared to the few poles (generally in the range of 5-15) obtained by direct moment matching techniques. Moreover, while direct moment matching techniques may not guarantee passivity [7], Krylov based methods have been proven to be passive by construction [10], [13]. The remainder of this section describes the Krylovsubspace based technique presented in [13] for the model order reduction of interconnect networks.

Consider a $p$-port linear subnetwork $\phi$ consisting of lumped RLC components, which can be expressed using time-domain modified nodal analysis equations (MNA) as [13], [75]:

$$
\begin{gathered}
\boldsymbol{C}_{\phi} \dot{\boldsymbol{v}_{\phi}}(t)+\boldsymbol{G}_{\phi} \boldsymbol{v}_{\phi}(t)=\boldsymbol{b}_{\phi} \boldsymbol{v}_{p}(t) \\
\boldsymbol{i}_{p}(t)=\boldsymbol{b}_{\phi}^{T} \boldsymbol{v}_{\phi}(t)
\end{gathered}
$$

where

$\boldsymbol{v}_{\phi}(t) \in \mathfrak{R}^{N_{\phi}}$ is the vector of node voltage waveforms appended by independent voltage source current and linear inductor currents. $\Re^{N_{\phi}}$ is the node-space of subnetwork $\phi$. 
$\boldsymbol{b}_{\phi} \in \mathfrak{R}^{N_{\phi} \times p}=\left[b_{i, j} \in\{0,-1\}\right]$ is a selector matrix, mapping port voltages into the node space $\left(\Re^{N_{\phi}}\right)$ of the subnetwork $\phi$, where $i \in\left\{1, \ldots, N_{\phi}\right\}, j \in\{1, \ldots, p\}$. $v_{p}(t)$ and $i_{p}(t)$ are port voltage and current vectors, respectively.

$\boldsymbol{C}_{\phi}, \boldsymbol{G}_{\phi} \in \Re^{N_{\phi} \times N_{\phi}}$ are constant matrices describing lumped memory and memoryless elements of subnetwork $\phi$, respectively. $\boldsymbol{C}_{\phi}, \boldsymbol{G}_{\phi}$ are obtained using the formulation in [13], such that:

$$
C_{\phi}=\left[\begin{array}{cc}
C_{a} & 0 \\
0 & C_{b}
\end{array}\right] ; \quad G_{\phi}=\left[\begin{array}{cc}
N_{\phi} & E_{\phi} \\
-E_{\phi}{ }^{T} & 0
\end{array}\right]
$$

where $N_{\phi}, C_{a}, C_{b}$ are matrices containing stamps of resistors, capacitors and inductors, respectively. Matrix $\boldsymbol{E}_{\phi} \in(1,-1,0)$ corresponds to current variables in a KCL formulation. Provided that the original network is composed of RLC elements, $N_{\phi}, C_{a}, C_{b}$ are symmetric nonnegative definite matrices. With this formulation, the resulting $\boldsymbol{C}_{\phi}$ is symmetric nonnegative definite and $\boldsymbol{G}_{\phi}+\boldsymbol{G}_{\phi}^{T} \geq 0$ [13].

Taking the Laplace transform of (3.1) and solving for port current variables, the admittance matrix of the p-port linear subnetwork can be obtained as

$$
\begin{aligned}
& \boldsymbol{Y}(s)=\boldsymbol{b}_{\phi}^{T}\left(\boldsymbol{I}_{N_{\phi}}-s \boldsymbol{A}_{\phi}\right)^{-1} \boldsymbol{R} ; \quad \boldsymbol{A}_{\phi}=-\boldsymbol{G}_{\phi}^{-1} \boldsymbol{C}_{\phi} ; \\
& \boldsymbol{R} \in \mathfrak{R}^{N_{\phi} \times p}=\boldsymbol{G}_{\phi}^{-1} \boldsymbol{b}_{\phi}=\left[\begin{array}{lll}
\boldsymbol{r}_{1} & \boldsymbol{r}_{2} & \ldots \\
\boldsymbol{r}_{p}
\end{array}\right]
\end{aligned}
$$


where $\boldsymbol{I}_{N_{\phi}} \in \Re^{N_{\phi} \times N_{\phi}}$ is an identity matrix. Next, the reduced order system of size $q$, with $q \ll N_{\phi}$ is generated by obtaining an orthonormal basis $Q \in \mathfrak{R}^{N_{\phi} \times q}$, using standard Arnoldi procedure [13], spanning the Krylov-subspace such that

$$
\begin{aligned}
\operatorname{ColumnSpace}(\boldsymbol{Q}) & =\operatorname{Kr}\left(\boldsymbol{A}_{\phi}, \boldsymbol{R}, q\right) \\
& =\operatorname{ColumnSpace}\left[\boldsymbol{R}, \boldsymbol{A}_{\phi} \boldsymbol{R}, \boldsymbol{A}_{\phi}{ }^{2} \boldsymbol{R}, \ldots \boldsymbol{A}_{\phi}{ }^{k-1} \boldsymbol{R}, \boldsymbol{A}_{\phi}{ }^{k} \tilde{\boldsymbol{R}}\right] \\
\tilde{\boldsymbol{R}}= & {\left[\begin{array}{llll}
\boldsymbol{r}_{1} & \boldsymbol{r}_{2} \ldots & \boldsymbol{r}_{l}
\end{array}\right] ; \quad k=\lfloor q / p\rfloor ; \quad l=q-k p }
\end{aligned}
$$

where the operator $L\rfloor$ represents the truncation to the nearest integer, towards zero [13]. For example, assume a 3 port characterization (i.e. $p=3$ ) and let $q=11$. In this case, we have $k=\lfloor 11 / 3\rfloor=3 ; \quad l=11-3 \times 3=2$ and (3.4) can be written as

$$
\operatorname{Kr}\left(\boldsymbol{A}_{\phi}, \boldsymbol{R}, q\right)=\text { ColumnSpace }\left[\boldsymbol{R}, \boldsymbol{A}_{\phi} \boldsymbol{R}, \boldsymbol{A}_{\phi}^{2} \boldsymbol{R}, \boldsymbol{A}_{\phi}{ }^{3} \boldsymbol{r}_{1}, \boldsymbol{A}_{\phi}{ }^{3} \boldsymbol{r}_{2}\right]
$$

Next, the reduced order admittance matrix is found by mapping the vector $\boldsymbol{v}_{\phi} \in \mathfrak{R}^{N_{\phi}}$ to a smaller vector $\hat{\boldsymbol{v}}_{\phi} \in \mathfrak{R}^{q}$ using the orthogonal matrix $Q$ and congruence transformation as

$$
\begin{gathered}
v_{\phi}=Q \hat{\boldsymbol{v}}_{\phi} \\
\hat{\boldsymbol{C}}_{\phi} \dot{\hat{\boldsymbol{v}}}_{\phi}=-\hat{\boldsymbol{G}}_{\phi} \hat{\boldsymbol{v}}_{\phi}+\hat{\boldsymbol{b}}_{\phi} \boldsymbol{v}_{p} \\
\boldsymbol{i}_{p}=\hat{\boldsymbol{b}}_{\phi}^{T} \hat{\boldsymbol{v}}_{\phi}
\end{gathered}
$$

where

$$
\hat{C}_{\phi}=Q^{T} C_{\phi} Q \quad \hat{G}_{\phi}=Q^{T} G_{\phi} Q \quad \hat{b}_{\phi}=Q^{T} b_{\phi}
$$


Therefore, in the Laplace-domain, the reduced-order admittance matrix can be written as

$$
\hat{\boldsymbol{Y}}(s)=\hat{\boldsymbol{b}}_{\phi}{ }^{T}\left(\boldsymbol{I}_{q}+s \hat{G}_{\phi}^{-1} \hat{\boldsymbol{C}}_{\phi}\right)^{-1} \hat{\boldsymbol{G}}_{\phi}^{-1} \hat{\boldsymbol{b}}_{\phi}
$$

Equation (3.8) represents the passive reduced-order model of the original system [13]. From (3.10) it can be seen that the eigenvalues of $-\hat{\boldsymbol{G}}_{\phi}^{-1} \hat{\boldsymbol{C}}_{\phi}$ are the reciprocal of the poles of the reduced order system. In the literature, the above algorithm is called PRIMA [13] and guarantees the passivity of the reduced model as long as the susceptance matrix $C_{\phi}$ is symmetric nonnegative definite and the conductance matrix satisfies the condition $\boldsymbol{G}_{\phi}+\boldsymbol{G}_{\phi}^{T} \geq 0$. MNA equations for RLC interconnect can be formulated such that the above conditions are satisfied [13].

The above scheme of reduction is referred to as the first-level model-reduction. However, recently it has become apparent that, although the first-level reduction algorithms are suitable for the reduction of large system, they may not produce a compact macromodel which is required for fast simulation. This has led to the focus on second-level reduction techniques based on truncated balanced realization (TBR) [71] - [74]. The next section covers the basics of TBR techniques.

\subsection{Truncated Balanced Realization}

It is well known that while the Krylov-subspace based techniques are very efficient for the reduction of large linear circuits, they may not produce a macromodel of optimal size. This has led to interest in the TBR algorithm which was developed in the area of control theory for the reduction of state-space systems with size of the order of few hundreds. As such, they cannot be directly 
used on large scale MNA equations resulting from interconnect networks. However, as the size of models resulting from first-level reduction algorithms such as PRIMA are of the order of few hundreds, TBR algorithms are ideally suited for the second-level reduction of these first-level reduced-order models. In this section, a review of the standard TBR algorithm [71] is presented followed by the passivity preserving TBR algorithm (PR-TBR) [72], [73].

\subsubsection{Standard TBR}

Consider a $n^{\text {th }}$ order state-space realization $\boldsymbol{A}, \boldsymbol{B}, \boldsymbol{C}, \boldsymbol{D}$ (not necessarily minimal)

$$
\begin{aligned}
& \dot{x}=A x+B u \\
& y=C x+D u
\end{aligned}
$$

where $\boldsymbol{x}$ is the state vector, $\boldsymbol{u}$ and $\boldsymbol{y}$ are the vectors containing the system inputs and outputs respectively. For example, the first-level reduced system represented by (3.8) - (3.10) can be directly casted in the form of state-space system of the form given by (3.11) - (3.12) as follows:

$$
\boldsymbol{A}=-\hat{\boldsymbol{C}}_{\phi}^{-1} \hat{\boldsymbol{G}}_{\phi} ; \quad \boldsymbol{B}=\hat{\boldsymbol{C}}_{\phi}^{-1} \hat{\boldsymbol{b}}_{\phi} ; \quad \boldsymbol{C}=\hat{\boldsymbol{b}}_{\phi}^{T}
$$

The transfer function of the above system in Laplace-domain can be written as

$$
\boldsymbol{G}(s)=\boldsymbol{C}(\boldsymbol{I} s-\boldsymbol{A})^{-1} \boldsymbol{B}+\boldsymbol{D}
$$

The concept of balanced truncation of a system was first introduced in [71]. The idea here is to take into consideration the inputs as well as the outputs of the system when deciding which states to retain in the reduced representation. This is equivalent to defining a certain subspace of the original state-space representation given by (3.11) - (3.12). Two important subspaces of interest are the controllable 
and observable subspaces. The controllable subspace is the set of states which could be obtained by a given input $\boldsymbol{u}(t)$ with zero initial state. The observable subspace is the set of states which when set as initial conditions can produce nonzero output $\boldsymbol{y}(t)$ with no external input. The TBR algorithm presented in [71] is based on computing the subspaces obtained from the controllability Grammian $\boldsymbol{W}_{c}$ and the observability Grammian $\boldsymbol{W}_{o}$. The eigenvectors of $\boldsymbol{W}_{c}$ span the controllable subspace while the eigenvectors of $\boldsymbol{W}_{o}$ span the observable subspace. These Grammians are evaluated by solving the following Lyapunov equations

$$
\begin{aligned}
& \boldsymbol{A} \boldsymbol{W}_{c}+\boldsymbol{W}_{c} \boldsymbol{A}^{T}+\boldsymbol{B} \boldsymbol{B}^{T}=0 \\
& \boldsymbol{A}^{T} \boldsymbol{W}_{o}+\boldsymbol{W}_{o} \boldsymbol{A}+\boldsymbol{C}^{T} \boldsymbol{C}=0
\end{aligned}
$$

It is known [71] that there exists an invertible transformation $\boldsymbol{T} \in \mathfrak{R}^{n \times n}$ such that the transformed system

$$
\hat{A}=T^{-1} A T ; \hat{B}=T^{-1} B ; \hat{C}=C T ; \hat{D}=D
$$

has controllability and observability Grammians of the form

$$
\hat{\boldsymbol{W}}_{c}=\boldsymbol{T}^{-1} \boldsymbol{W}_{c} \boldsymbol{T}^{-T}=\hat{\boldsymbol{W}}_{o}=\boldsymbol{T}^{T} \boldsymbol{W}_{o} \boldsymbol{T}=\Sigma
$$

where $\Sigma$ is positive definite diagonal matrix of the form

$$
\begin{gathered}
\Sigma=\operatorname{diag}\left(\sigma_{1}, \ldots, \sigma_{n}\right) \\
\sigma_{1} \geq \sigma_{2} \geq \ldots \geq \sigma_{k} \geq \sigma_{k+1} \ldots \geq \sigma_{n}
\end{gathered}
$$

with $\sigma_{i}=\left(\lambda_{i}\left(\boldsymbol{W}_{c} \boldsymbol{W}_{o}\right)\right)^{1 / 2}$. Here $\lambda_{i}\left(\boldsymbol{W}_{c} \boldsymbol{W}_{o}\right)$ denotes the $i^{\text {th }}$ eigenvalue of $\boldsymbol{W}_{c} \boldsymbol{W}_{o}$. 
The reduced model is obtained by truncating the least controllable-observable states. For instance, to obtain a reduced model of order $k(k \leq n)$, partition $\hat{\boldsymbol{A}}, \hat{\boldsymbol{B}}, \hat{\boldsymbol{C}}, \hat{\boldsymbol{D}}$ as $[71]$

$$
\left[\begin{array}{c|c}
\hat{\boldsymbol{A}} & \hat{\boldsymbol{B}} \\
\hline \hat{\boldsymbol{C}} & \hat{\boldsymbol{D}}
\end{array}\right]=\left[\begin{array}{cc|c}
\hat{\boldsymbol{A}}_{11} & \hat{\boldsymbol{A}}_{12} & \hat{\boldsymbol{B}}_{1} \\
\hat{\boldsymbol{A}}_{21} & \hat{\boldsymbol{A}}_{22} & \hat{\boldsymbol{B}}_{2} \\
\hline \hat{\boldsymbol{C}}_{1} & \hat{\boldsymbol{C}}_{2} & \hat{\boldsymbol{D}}
\end{array}\right]
$$

where $\hat{\boldsymbol{A}}_{11} \in \mathfrak{R}^{k \times k}$ and $\hat{\boldsymbol{A}}_{22} \in \Re^{(n-k) \times(n-k)}$, then truncate the (n-k) least controllable-observable states to obtain the reduced model

$$
\boldsymbol{G}_{r}(s)=\hat{\boldsymbol{C}}_{1}\left(\boldsymbol{I} s-\hat{\boldsymbol{A}}_{11}\right)^{-1} \hat{\boldsymbol{B}}_{1}+\hat{\boldsymbol{D}}
$$

However, the reduced system in (3.22) does not guarantee the passivity of the macromodel. In the next subsection, passivity guaranteeing TBR algorithm is presented.

\subsubsection{PR-TBR based Second Level Reduction}

It has been shown in [72], [73] that the above reduced model (3.22) can be made guaranteed passive by obtaining controllability Grammian $\left(\boldsymbol{W}_{c}\right)$ and observability Grammian ( $\boldsymbol{W}_{o}$ ) via solving Lur e equations and its dual, instead of Lyapunov equations (3.15), (3.16). Here, the first set of Lur e equations

$$
\begin{gathered}
\boldsymbol{A}^{T} \boldsymbol{W}_{o}+\boldsymbol{W}_{o} \boldsymbol{A}=-\boldsymbol{K}_{o}^{T} \boldsymbol{K}_{o} \\
\boldsymbol{W}_{o} \boldsymbol{B}-\boldsymbol{C}^{T}=-\boldsymbol{K}_{o}^{T} \boldsymbol{J}_{o} \\
\boldsymbol{J}_{o}^{T} \boldsymbol{J}_{o}=\boldsymbol{D}+\boldsymbol{D}^{T}
\end{gathered}
$$


is solved to obtain the observability Grammian $\boldsymbol{W}_{o} \geq 0$. The solution of (3.23) is generally obtained by solving the following algebraic riccati equation (ARE) [42]

$$
\left(\boldsymbol{A}^{T}-\boldsymbol{C}^{T} \boldsymbol{R}^{-1} \boldsymbol{B}^{T}\right) \boldsymbol{W}_{o}+\boldsymbol{W}_{o}\left(\boldsymbol{A}-\boldsymbol{B} \boldsymbol{R}^{-1} \boldsymbol{C}\right)+\boldsymbol{W}_{o} \boldsymbol{B} \boldsymbol{R}^{-1} \boldsymbol{B}^{T} \boldsymbol{W}_{o}+\boldsymbol{C}^{T} \boldsymbol{R}^{-1} \boldsymbol{C}=0
$$

where $\boldsymbol{R}=\boldsymbol{D}+\boldsymbol{D}^{T}$. Next, the dual set of Lur e equations

$$
\begin{gathered}
\boldsymbol{A} \boldsymbol{W}_{c}+\boldsymbol{W}_{c} \boldsymbol{A}^{T}=-\boldsymbol{K}_{c} \boldsymbol{K}_{c}^{T} \\
\boldsymbol{W}_{c} \boldsymbol{C}^{T}-\boldsymbol{B}=-\boldsymbol{K}_{c} \boldsymbol{J}_{c}^{T} \\
\boldsymbol{J}_{c} \boldsymbol{J}_{c}^{T}=\boldsymbol{D}+\boldsymbol{D}^{T}
\end{gathered}
$$

is solved to obtain the controllability Grammian $\boldsymbol{W}_{c} \geq 0$. The solution of (3.25) is generally obtained by solving the following algebraic riccati equation (ARE)

$$
\left(A-B R^{-1} C\right) W_{c}+W_{c}\left(A^{T}-C^{T} R^{-1} B^{T}\right)+W_{c} C^{T} R^{-1} C W_{c}+B R^{-1} B^{T}=0
$$

Solution of the AREs (3.24) and (3.26) is generally obtained by invariant subspace method [76], where the solution is obtained from the invariant subspace of the associated Hamiltonian matrix. This requires the schur decomposition of two Hamiltonian matrices whose size is twice that of the state-space system, i.e. $2 n$. However, the computational cost of this method is $203 n^{3}$ [77], which implies that the total cost of the above scheme will be $406 n^{3}$. This can be excessive for practical purposes and needs new algorithms to reduce the involved cost.

\subsection{Conclusion}

This chapter has reviewed model reduction algorithms for interconnect networks. First level reduction based on direct moment matching and indirect moment 
matching have been discussed. Second-level reduction algorithms based on TBR algorithm have been described. Based on the review material in chapter 2 and 3 , we can summarise what is to come in the rest of the chapters as follows.

As pointed out in Section 2.4, a critical issue in handling tabulated networks is ensuring the passivity of the macromodel. The passivity ensuring techniques presented in Section 2.5.1 and Section 2.5.2 become prohibitively CPU expensive as the order of the macromodel increases. In order to address these difficulties, efficient algorithms for ensuring the passivity of the macromodel of tabulated networks are presented in chapters 4 and 5. They have an advantage over algorithms in Section 2.5, due to their robustness and computational efficiency.

Additionally, another main concern with the passivity enforcement algorithms is that new regions of passivity violation are introduced during the iterations. To overcome this, a novel algorithm is presented in chapter 6 for small passivity violations, involving a guaranteed direction during passivity compensation. This reduces the time required for enforcing the passivity of the macromodel.

Also, as discussed in chapter 1, there is a growing tendency towards designing signal paths consisting of subnetworks described by tabulated data. In view of this, there is a need for an efficient model reduction algorithm like PRIMA for the reduction of the overall MNA equations of the signal path consisting of regular interconnect models (such as RLC model) plus general state-space macromodels of subnetworks described by tabulated data. This is because PRIMA type of firstlevel algorithm, in its original form, is not suitable for including embedded modules described by arbitrary state-space representation. In chapter 7 , a new algorithm to overcome the above problem is described, followed by a new technique to reduce the cost of second-level reduction algorithm. 


\section{CHAPTER 4}

\section{Passive Macromodeling of Subnetworks Characterized by Tabulated Data}

It was pointed out in chapters 1 and 2 that, one of the critical issues in modern design arena is representing the tabulated network in a circuit simulation environment. Important applications of tabulated network include highspeed interconnects, packages, vias, nonuniform transmission lines, on-chip passive components, and high-frequency microwave devices. However, transient analysis of tabulated networks is a challenging problem due to the well known mixed time/frequency difficulty, since the tabulated data is in frequency-domain, while the terminations are described in time-domain. Many algorithms [29] - [38], [44] - [46] have been proposed to address this issue. These algorithms essentially approximate the tabulated data by rational functions and subsequently synthesize a SPICE compatible macromodel/netlist from such an approximation. However, the primary challenge while modeling tabulated networks is to ensure the passivity of the macromodel.

Many passivity preservation algorithms for tabulated data can be found in the literature [31], [33] - [38], [41], [42], [44] - [46]. Algorithms such as the ones based on convex optimization [34], [35] can guarantee the passivity of the macromodel. However, they can be CPU expensive (since the associated computational complexity is in the range of $q^{4}$ to $q^{6}$, where $q$ is the order of 
the state-space matrix) and may not be practically feasible. On the other hand, approaches such as the ones in [29] - [31], [41], [42], [44] - [46] are computationally fast; however, they may not strictly guarantee the macromodel passivity. In order to address this issue, an efficient four-step algorithm is presented in this chapter for the passive macromodeling of tabulated data. The tabulated data can be in admittance $(\mathrm{Y})$, impedance $(\mathrm{Z})$ or scattering $(\mathrm{S})$ form. For the ease of presentation, the description is restricted to the scattering parameters.

\subsection{Problem Formulation}

The scattering $(S)$ parameters are generally used to characterize multiport subnetworks at higher frequencies, by relating the incident travelling waves (a) and the reflected travelling waves $(\boldsymbol{b})$ as

$$
b=S a
$$

The rational approximation of $S$-parameter data of a $m$-port subnetwork can be written as

$$
S(s)=\left[S_{i j}(s)\right] ; \quad S_{i j}(s)=\frac{\left(a_{0}^{(i, j)}+\ldots+a_{l}^{(i, j)} s^{l}\right)}{\left(b_{0}^{(i, j)}+\ldots+b_{n}^{(i, j)} s^{n}\right)} ; \quad(i, j \in 1 \ldots m)
$$

However, one of the major challenge while obtaining the approximation (4.2) is the passivity of the multiport macromodel. Passivity is an important property to be satisfied by a macromodel [3], [5], [13], [15], [24], because stable but non-passive models may lead to unstable systems when connected to other passive components. On the other hand, a passive macromodel, when terminated with any arbitrary passive load always guarantees the stability of the overall resulting network. To illustrate this point, consider a simple, single-port 
second-order macromodel shown in Fig. 4.1(a). The macromodel is stable but not passive. When this macromodel is terminated with the passive load (Fig. 4.1(b)), the overall network ends up having unstable poles.

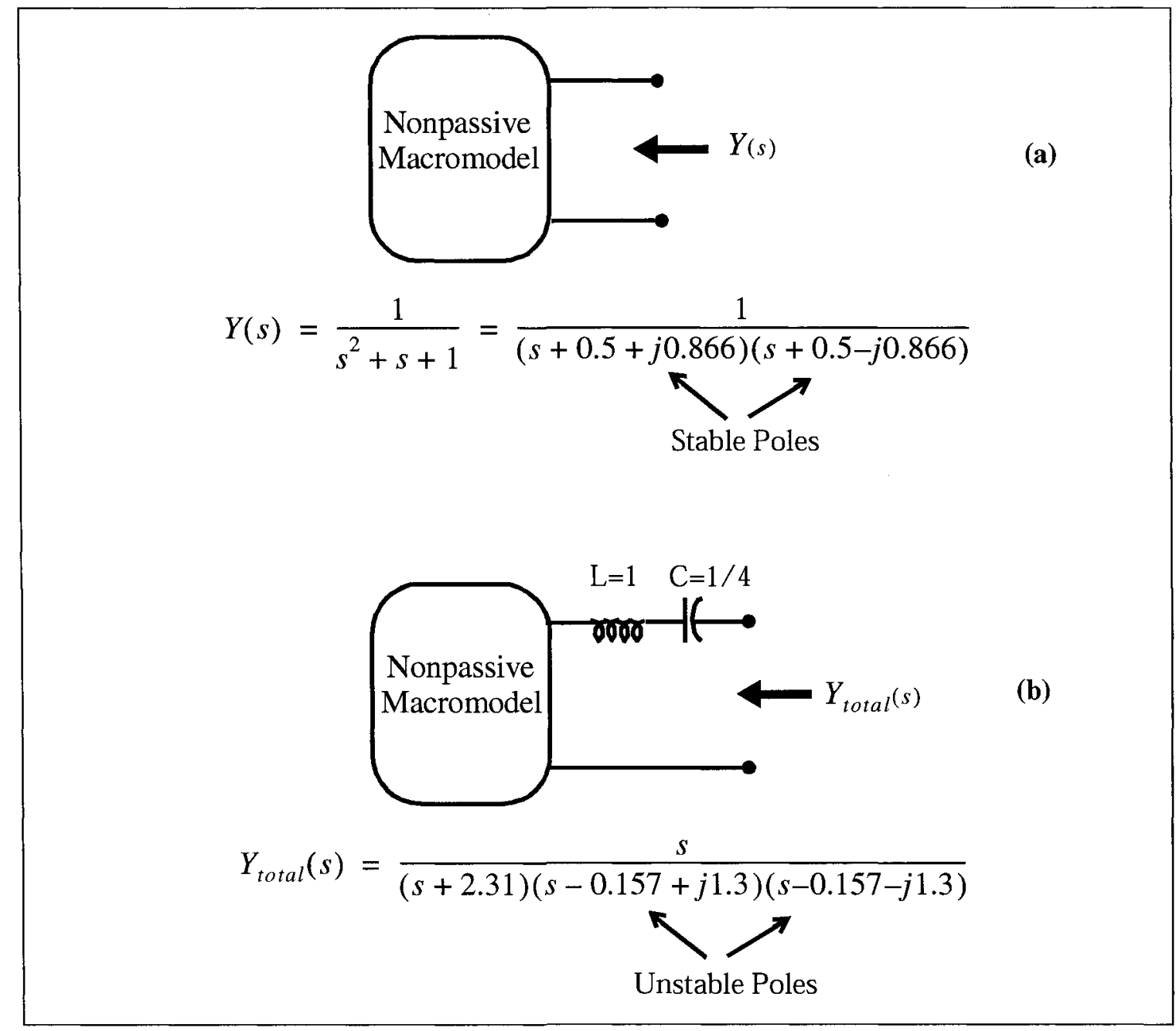

Fig. 4.1. Illustration of significance of passivity: (a) Macromodel alone - stable (b) Macromodel plus passive load - unstable

A network with scattering matrix $\boldsymbol{S}(s)$ as defined by rational function (4.2) is passive iff it is bounded real [69]. For a network to be bounded real, it must satisfy the following conditions [69] 
(a) No element of $\boldsymbol{S}(s)$ possesses a pole in $\operatorname{Re}(s) \geq 0$

(b) Each entry of $\boldsymbol{S}(s)$ is a real rational function, i.e. a ratio of two polynomials with real coefficients.

(c) $\boldsymbol{I}-S^{H}(j \omega) \boldsymbol{S}(j \omega) \geq 0$ for all $\omega \in \mathfrak{R} \cup \infty$. Here $H$ represents the Hermitian operator.

Condition (a) can be readily satisfied during the approximation process. To satisfy condition (b), complex poles/residues are always considered along with their conjugates leading to only real coefficients in rational functions of $S(s)$. However, ensuring condition (c) is not easy. A straight-forward formulation for this purpose can lead to computationally expensive nonlinear optimization, which may suffer from non-convergence.

In order to address this challenge, a new algorithm for passive macromodeling of $S$-parameter based high-speed subnetworks is presented in the ensuing sections. The algorithm involves systematic passivity check, detection of the band-width of passivity violating regions, and fast passivity compensation.

\subsection{Proposed Passive Macromodeling Algorithm}

The proposed algorithm for passive macromodeling of tabulated data consists of four main steps [47], [50] - [53] (illustrated in Fig. 4.2):

1. In the first step, a multiport rational function approximation of the tabulated data is obtained, using the vector-fitting [26] technique described in [27], [28]. This rational function approximation is converted into state-space approximation using Jordan canonical form [78] - [80]. 
2. In the second step, the resulting state-space macromodel is checked for passivity, using algebraic tests based on the eigenanalysis of Hamiltonian matrix.

3. In case the macromodel fails the passivity check test, in the third step, the passivity violating regions are identified and the extent of violation is quantified.

4. In the fourth step, passivity of the nonpassive macromodel is enforced by perturbing the residue matrix, based on the information from the third step.

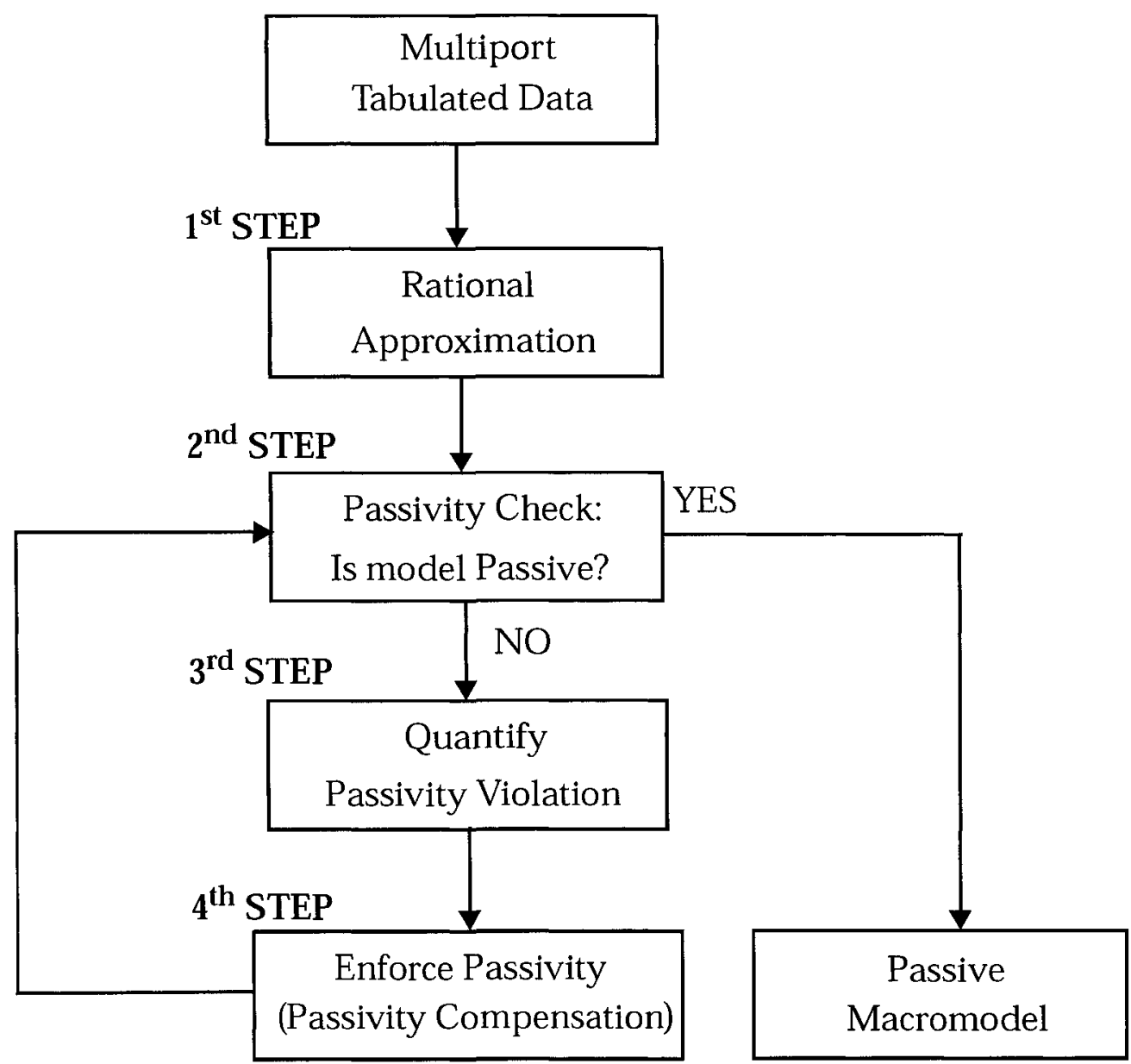

Fig. 4.2. Flow diagram of the proposed passive macromodeling algorithm 
The details of the above steps are given below.

\subsubsection{First Step: Approximation}

In this step, the tabulated data of each element of the scattering matrix $S(s)$ of a $m$-port subnetwork is approximated by the pole-residue form, using vector-fitting technique, as described in [26] - [28]

$$
S_{i j}(s)=\sum_{i=1}^{n} \frac{k_{i}^{i, j}}{s-p_{i}}+d^{i, j} ; \quad i, j=1,2, \ldots m
$$

where the residues $\left(k_{i}\right)$ and poles $\left(p_{i}\right)$ can be real or complex conjugate pairs, $n$ is the number of poles and residues, $d$ is the real constant. In order that the model is bounded real (passive) at $\omega=\infty$, a constrained vector-fitting is used, whereby it is guaranteed that the norm of the matrix of direct coupling constants $\boldsymbol{D}=\left[d^{i, j}\right] ; \quad(i, j \in 1 \ldots m)$ is less than one [52].

Next, for the purpose of passivity checking and enforcement, the pole-residue model (4.3) is translated into the state-space form. In general, a set of first-order differential equations in the state-space domain can be described as

$$
\begin{aligned}
& \dot{\boldsymbol{x}}(t)=\boldsymbol{A} \boldsymbol{x}(t)+\boldsymbol{B} \boldsymbol{a}(t) \\
& \boldsymbol{b}(t)=\boldsymbol{C} \boldsymbol{x}(t)+\boldsymbol{D} \boldsymbol{a}(t)
\end{aligned}
$$

where $\boldsymbol{x}(t) \in \mathfrak{R}^{q \times 1}$ (with $q=n m$ ) is the state vector, $\boldsymbol{a}(t) \in \mathfrak{R}^{m \times 1}$ is the input vector. $\boldsymbol{A} \in \mathfrak{R}^{q \times q}$ is the state-matrix, $\boldsymbol{B} \in \mathfrak{R}^{q \times m}$ is the matrix that relates the inputs to state-variables, $C \in \mathfrak{R}^{m \times q}$ is the matrix relating state variables to the 
outputs $\left(\boldsymbol{b}(t) \in \mathfrak{R}^{m \times 1}\right)$ and $\boldsymbol{D} \in \mathfrak{R}^{m \times m}$ is a matrix of direct coupling constants. The relationship between the input $\boldsymbol{a}(s)$ and output $\boldsymbol{b}(s)$ in frequency domain can be written as

$$
\boldsymbol{S}(s)=\boldsymbol{C}(s \boldsymbol{I}-\boldsymbol{A})^{-1} \boldsymbol{B}+\boldsymbol{D}
$$

It is assumed that the matrix $\boldsymbol{A}$ has no imaginary and unstable eigenvalues, and the matrix $D$ (constituted by the direct coupling constants $d^{i, j}$ ) has the norm less than one.

\subsubsection{Second Step: Passivity Check}

Since no constraints are applied during the approximation process of (4.3) or (4.4), the resulting macromodel needs to be tested for passivity and compensated if required. The macromodel obtained from the first step satisfies conditions (a) and (b). The only remaining test for passivity check is condition (c). Condition (c) can be equivalently expressed as

$$
\|S(j \omega)\|_{2} \leq 1 \text { for all } \omega \in \Re \cup \infty .
$$

The most straight forward way to check condition (c) for ascertaining the passivity of the macromodel, is to plot $\|S(j \omega)\|_{2}$ versus $\omega$ and observe if it crosses the value of one at any frequency point [70]. However, it is impossible to perform this test up to $\omega=\infty$. Hence, what is desired is some kind of algebraic test, in terms of the macromodel state-space matrices, that is independent of frequency. For this purpose, following theorems from the control area are used to check the passivity and identify the locations of passivity violations 
Theorem 1: The state-space system $(\boldsymbol{A}, \boldsymbol{B}, \boldsymbol{C}, \boldsymbol{D})$ is bounded real (passive) iff the following Hamiltonian Matrix (M) [70], [47], [50] - [53] has no imaginary eigenvalues,

$$
\boldsymbol{M}=\left[\begin{array}{cc}
\boldsymbol{A}+\boldsymbol{B}\left(\boldsymbol{I}-\boldsymbol{D}^{T} \boldsymbol{D}\right)^{-1} \boldsymbol{D}^{T} \boldsymbol{C} & \boldsymbol{B}\left(\boldsymbol{I}-\boldsymbol{D}^{T} \boldsymbol{D}\right)^{-1} \boldsymbol{B}^{T} \\
-C^{T}\left(\boldsymbol{I}-\boldsymbol{D} \boldsymbol{D}^{T}\right)^{-1} \boldsymbol{C} & -\boldsymbol{A}^{T}-C^{T} \boldsymbol{D}\left(\boldsymbol{I}-\boldsymbol{D}^{T} \boldsymbol{D}\right)^{-1} \boldsymbol{B}^{T}
\end{array}\right]
$$

The advantage of the above theorem is that, the formulation of the Hamiltonian matrix (4.7) is independent of frequency. Hence, if no imaginary eigenvalues are found, it automatically implies that the macromodel is passive. However, using the traditional method of frequency-sweep of the norm of scattering matrix $S(j \omega)$ would have required the sweep from 0 to $\infty$, to detect if the norm is greater than one.

If the macromodel is found to be non-passive (i.e. if there exist imaginary eigenvalues of Hamiltonian matrix (4.7)), the following theorem helps in identifying the exact locations of passivity violation.

Theorem 2: $\boldsymbol{S}\left(j \omega_{0}\right)$ has a maximum singular value equal to one (i.e. the norm equal to one) if $j \omega_{0}$ is an imaginary eigenvalue of $\boldsymbol{M}$, provided $\boldsymbol{A}$ has no imaginary eigenvalues and $\boldsymbol{D}$ does not have a singular value equal to one [81], [47], [50] - [53].

Proof of Theorem 2: In order to relate the imaginary eigenvalues of Hamiltonian matrix $\boldsymbol{M}$ with the locations where the singular value of $S(j \omega)$ become equal to one, consider that $j \omega_{0}$ is an imaginary eigenvalue of $\boldsymbol{M}$, i.e. 


$$
\left.\boldsymbol{M}[\boldsymbol{e}]=j \omega_{0}[\boldsymbol{e}] \boldsymbol{f}\right]
$$

where $\left[\begin{array}{ll}e^{T} & f^{T}\end{array}\right]^{T}$ is the eigenvector of $\boldsymbol{M}$ corresponding to its eigenvalue $j \omega_{0}$.

Substituting $\boldsymbol{M}$ from (4.7), (4.8) can be written as

$$
\left[\begin{array}{cc}
\boldsymbol{A}+\boldsymbol{B}\left(\boldsymbol{I}-\boldsymbol{D}^{T} \boldsymbol{D}\right)^{-1} \boldsymbol{D}^{T} \boldsymbol{C} & \boldsymbol{B}\left(\boldsymbol{I}-\boldsymbol{D}^{T} \boldsymbol{D}\right)^{-1} \boldsymbol{B}^{T} \\
-\boldsymbol{C}^{T}\left(\boldsymbol{I}-\boldsymbol{D} \boldsymbol{D}^{T}\right)^{-1} \boldsymbol{C} & -\boldsymbol{A}^{T}-\boldsymbol{C}^{T} \boldsymbol{D}\left(\boldsymbol{I}-\boldsymbol{D}^{T} \boldsymbol{D}\right)^{-1} \boldsymbol{B}^{T}
\end{array}\right][\boldsymbol{f}]=j \omega_{0}[\boldsymbol{e}]
$$

After some mathematical manipulations (4.9) can be written as

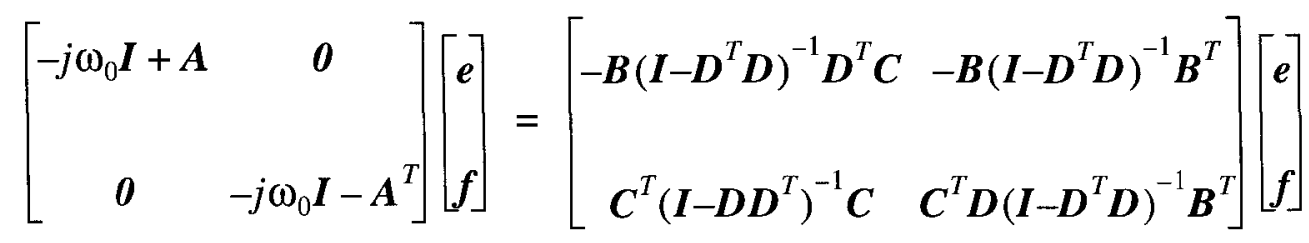

Next, introducing vector $\boldsymbol{h}$ and $\boldsymbol{g}$ as follows

$$
\begin{aligned}
& \boldsymbol{h}=\left(\boldsymbol{I}-\boldsymbol{D}^{T} \boldsymbol{D}\right)^{-1} \boldsymbol{D}^{T} \boldsymbol{C e}+\left(\boldsymbol{I}-\boldsymbol{D}^{T} \boldsymbol{D}\right)^{-1} \boldsymbol{B}^{T} \boldsymbol{f} \\
& \boldsymbol{g}=\left(\boldsymbol{I}-\boldsymbol{D} \boldsymbol{D}^{T}\right)^{-1} \mathrm{Ce}+\boldsymbol{D}\left(\boldsymbol{I}-\boldsymbol{D}^{T} \boldsymbol{D}\right)^{-1} \boldsymbol{B}^{T} \boldsymbol{f}
\end{aligned}
$$

(4.10) can be represented as

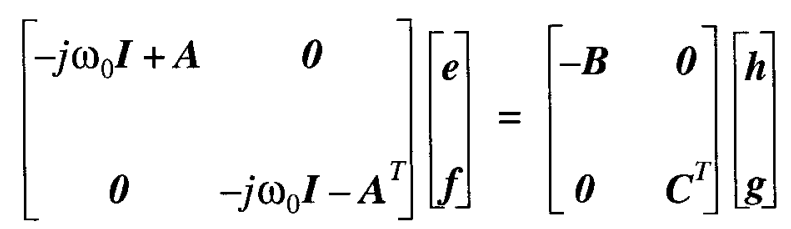

Combining (4.11) and (4.12) we get 


$$
\left[\begin{array}{l}
\boldsymbol{h} \\
\boldsymbol{g}
\end{array}\right]=\left[\begin{array}{cc}
\left(\boldsymbol{I}-\boldsymbol{D}^{T} \boldsymbol{D}\right)^{-1} \boldsymbol{D}^{T} \boldsymbol{C} & \left(\boldsymbol{I}-\boldsymbol{D}^{T} \boldsymbol{D}\right)^{-1} \boldsymbol{B}^{T} \\
\left(\boldsymbol{I}-\boldsymbol{D} \boldsymbol{D}^{T}\right)^{-1} \boldsymbol{C} & \boldsymbol{D}\left(\boldsymbol{I}-\boldsymbol{D}^{T} \boldsymbol{D}\right)^{-1} \boldsymbol{B}^{T}
\end{array}\right]\left[\begin{array}{l}
\boldsymbol{e} \\
\boldsymbol{f}
\end{array}\right]
$$

After certain mathematical manipulations and noting the following fact about inverse of a partition matrix $\boldsymbol{T}$

$$
\begin{gathered}
\boldsymbol{T}^{-1}=\left[\begin{array}{ll}
\boldsymbol{T}_{11} & \boldsymbol{T}_{12} \\
\boldsymbol{T}_{21} & \boldsymbol{T}_{22}
\end{array}\right]^{-1}=\left[\begin{array}{ll}
J & K \\
L & N
\end{array}\right] ; \\
J=\left(\boldsymbol{T}_{11}-\boldsymbol{T}_{12} \boldsymbol{T}_{22}{ }^{-1} \boldsymbol{T}_{21}\right)^{-1} \\
\boldsymbol{N}=\left(\boldsymbol{T}_{22}-\boldsymbol{T}_{21} \boldsymbol{T}_{11}{ }^{-1} \boldsymbol{T}_{12}\right)^{-1} \\
\boldsymbol{K}=-\boldsymbol{T}_{11}{ }^{-1} \boldsymbol{T}_{12}\left(\boldsymbol{T}_{22}-\boldsymbol{T}_{21} \boldsymbol{T}_{11}^{-1} \boldsymbol{T}_{12}\right)^{-1} \\
L=-\left(\boldsymbol{T}_{22}-\boldsymbol{T}_{21} \boldsymbol{T}_{11}^{-1} \boldsymbol{T}_{12}\right)^{-1} \boldsymbol{T}_{21} \boldsymbol{T}_{11}{ }^{-1}
\end{gathered}
$$

we can write (4.14) as

$$
\left[\begin{array}{l}
\boldsymbol{h} \\
\boldsymbol{g}
\end{array}\right]=\left[\begin{array}{cc}
-\boldsymbol{D} & \boldsymbol{I} \\
\boldsymbol{I} & -\boldsymbol{D}^{T}
\end{array}\right]^{-1}\left[\begin{array}{cc}
\boldsymbol{C} & 0 \\
\boldsymbol{O} & \boldsymbol{B}^{T}
\end{array}\right]\left[\begin{array}{l}
\boldsymbol{e} \\
\boldsymbol{f}
\end{array}\right]
$$

Now substituting (4.13) into (4.16) we get

$$
\left[\begin{array}{l}
\boldsymbol{h} \\
\boldsymbol{g}
\end{array}\right]=\left[\begin{array}{cc}
-\boldsymbol{D} & \boldsymbol{I} \\
\boldsymbol{I} & -\boldsymbol{D}^{T}
\end{array}\right]^{-1}\left[\begin{array}{cc}
\boldsymbol{C} & \boldsymbol{0} \\
\boldsymbol{0} & \boldsymbol{B}^{T}
\end{array}\right]\left[\begin{array}{cc}
-j \omega_{0} \boldsymbol{I}+\boldsymbol{A} & \boldsymbol{0} \\
\boldsymbol{0} & -j \omega_{0} \boldsymbol{I}-\boldsymbol{A}^{T}
\end{array}\right]^{-1}\left[\begin{array}{cc}
-\boldsymbol{B} & \boldsymbol{0} \\
\boldsymbol{0} & C^{T}
\end{array}\right]\left[\begin{array}{l}
\boldsymbol{h} \\
\boldsymbol{g}
\end{array}\right]
$$

After further simplification (4.17) can be expressed as 


$$
\left[\begin{array}{l}
\boldsymbol{g} \\
\boldsymbol{h}
\end{array}\right]=\left[\begin{array}{cc}
\boldsymbol{C}\left(j \omega_{0} \boldsymbol{I}-\boldsymbol{A}\right)^{-1} \boldsymbol{B}+\boldsymbol{D} & 0 \\
0 & \boldsymbol{B}^{T}\left(-j \omega_{0} \boldsymbol{I}-\boldsymbol{A}^{T}\right)^{-1} \boldsymbol{C}^{T}+\boldsymbol{D}^{T}
\end{array}\right]\left[\begin{array}{l}
\boldsymbol{h} \\
\boldsymbol{g}
\end{array}\right]
$$

or

$$
\begin{gathered}
\left(\boldsymbol{C}\left(j \omega_{0} \boldsymbol{I}-\boldsymbol{A}\right)^{-1} \boldsymbol{B}+\boldsymbol{D}\right) \boldsymbol{h}=\boldsymbol{g} \\
\left(\boldsymbol{B}^{T}\left(-j \omega_{0} \boldsymbol{I}-\boldsymbol{A}^{T}\right)^{-1} \boldsymbol{C}^{T}+\boldsymbol{D}^{T}\right) \boldsymbol{g}=\boldsymbol{h}
\end{gathered}
$$

Substituting $\boldsymbol{S}\left(j \omega_{0}\right)$ from (4.5) in (4.19) and (4.20) we get

$$
S\left(j \omega_{0}\right) \boldsymbol{h}=\boldsymbol{g}
$$

and

$$
\boldsymbol{S}\left(j \omega_{0}^{*}\right)^{T} \boldsymbol{g}=\boldsymbol{h} ; \Rightarrow \boldsymbol{S}\left(j \omega_{0}\right)^{H} \boldsymbol{g}=\boldsymbol{h}
$$

where, $H$ is the Hermitian operator. From (4.21) and (4.22) it is evident that at $j \omega_{0}$ the singular value of $\boldsymbol{S}(j \omega)$ is one.

Using a similar approach, the proof of corollary of the Theorem-2 can be obtained as follows.

Corollary of Theorem 2: $j \omega_{0}$ is an imaginary eigenvalue of $\boldsymbol{M}$ if, $\boldsymbol{S}\left(j \omega_{0}\right)$ has a maximum singular value equal to one (i.e. the norm equal to one), provided $\boldsymbol{A}$ has no imaginary eigenvalues and $\boldsymbol{D}$ does not have a singular value equal to one.

Proof of Corollary of Theorem 2: Consider that $\boldsymbol{S}\left(j \omega_{0}\right)$ has a maximum singular value equal to one, i.e. 


$$
\begin{gathered}
\boldsymbol{S}\left(j \omega_{0}\right) \boldsymbol{h}=\boldsymbol{g} \\
\boldsymbol{S}\left(j \omega_{0}\right)^{H} \boldsymbol{g}=\boldsymbol{h}
\end{gathered}
$$

Substituting $\boldsymbol{S}\left(j \omega_{0}\right)$ from (4.5) in (4.23) and (4.24) we get

$$
\begin{gathered}
\left(\boldsymbol{C}\left(j \omega_{0} \boldsymbol{I}-\boldsymbol{A}\right)^{-1} \boldsymbol{B}+\boldsymbol{D}\right) \boldsymbol{h}=\boldsymbol{g} \\
\left(\boldsymbol{B}^{T}\left(-j \omega_{0} \boldsymbol{I}-\boldsymbol{A}^{T}\right)^{-1} \boldsymbol{C}^{T}+\boldsymbol{D}^{T}\right) \boldsymbol{g}=\boldsymbol{h}
\end{gathered}
$$

Next, introducing vector $\boldsymbol{u}$ and $\boldsymbol{v}$ as follows

$$
\begin{gathered}
\boldsymbol{u}=\left(j \omega_{0} \boldsymbol{I}-\boldsymbol{A}\right)^{-1} \boldsymbol{B} \boldsymbol{h} \\
\boldsymbol{v}=\left(-j \omega_{0} \boldsymbol{I}-\boldsymbol{A}^{T}\right)^{-1} \boldsymbol{C}^{T} \boldsymbol{g}
\end{gathered}
$$

Substituting (4.27) and (4.28) in (4.25) and (4.26), we get

$$
\left[\begin{array}{l}
\boldsymbol{h} \\
\boldsymbol{g}
\end{array}\right]=\left[\begin{array}{cc}
-\boldsymbol{D} & \boldsymbol{I} \\
\boldsymbol{I} & -\boldsymbol{D}^{T}
\end{array}\right]^{-1}\left[\begin{array}{cc}
\boldsymbol{C} & 0 \\
0 & B^{T}
\end{array}\right]\left[\begin{array}{l}
\boldsymbol{u} \\
v
\end{array}\right]
$$

From (4.27) and (4.28) we have

$$
\left[\begin{array}{l}
\boldsymbol{h} \\
\boldsymbol{g}
\end{array}\right]=\left[\begin{array}{ll}
\boldsymbol{B} & \boldsymbol{0} \\
\boldsymbol{0} & \boldsymbol{C}^{T}
\end{array}\right]^{-1}\left[\begin{array}{cc}
\left(j \omega_{0} \boldsymbol{I}-\boldsymbol{A}\right) & \boldsymbol{0} \\
\boldsymbol{0} & \left(-j \omega_{0} \boldsymbol{I}-\boldsymbol{A}^{T}\right)
\end{array}\right]\left[\begin{array}{l}
\boldsymbol{u} \\
\boldsymbol{v}
\end{array}\right]
$$

Substituting (4.30) in (4.29) we get

$$
\left[\begin{array}{cc}
\left(j \omega_{0} I-\boldsymbol{A}\right) & \boldsymbol{0} \\
\boldsymbol{0} & \left(-j \omega_{0} \boldsymbol{I}-\boldsymbol{A}^{T}\right)
\end{array}\right]\left[\begin{array}{l}
\boldsymbol{u} \\
\boldsymbol{v}
\end{array}\right]=\left[\begin{array}{cc}
\boldsymbol{B} & 0 \\
\boldsymbol{0} & \boldsymbol{C}^{T}
\end{array}\right]\left[\begin{array}{cc}
-\boldsymbol{D} & \boldsymbol{I} \\
\boldsymbol{I} & -\boldsymbol{D}^{T}
\end{array}\right]^{-1}\left[\begin{array}{cc}
\boldsymbol{C} & \boldsymbol{0} \\
\boldsymbol{0} & \boldsymbol{B}^{T}
\end{array}\right]\left[\begin{array}{l}
\boldsymbol{u} \\
v
\end{array}\right]
$$


or

$$
\left\{\left[\begin{array}{cc}
\boldsymbol{A} & \boldsymbol{0} \\
\boldsymbol{0} & -\boldsymbol{A}^{T}
\end{array}\right]+\left[\begin{array}{cc}
\boldsymbol{B} & \boldsymbol{0} \\
\boldsymbol{0} & -\boldsymbol{C}^{T}
\end{array}\right]\left[\begin{array}{cc}
-\boldsymbol{D} & \boldsymbol{I} \\
\boldsymbol{I} & -\boldsymbol{D}^{T}
\end{array}\right]^{-1}\left[\begin{array}{cc}
\boldsymbol{C} & 0 \\
\boldsymbol{0} & \boldsymbol{B}^{T}
\end{array}\right]\right\}\left[\begin{array}{l}
\boldsymbol{u} \\
\boldsymbol{v}
\end{array}\right]=j \omega_{0}\left[\begin{array}{l}
\boldsymbol{u} \\
\boldsymbol{v}
\end{array}\right]
$$

or, using (4.7)

$$
\boldsymbol{M}\left[\begin{array}{l}
\boldsymbol{u} \\
\boldsymbol{v}
\end{array}\right]=j \omega_{0}\left[\begin{array}{l}
\boldsymbol{u} \\
\boldsymbol{v}
\end{array}\right]
$$

This proves the corollary of theorem 2 .

From the above theorem and its corollary, it is evident that an imaginary eigenvalue of Hamiltonian matrix $\boldsymbol{M}$ corresponds to the frequency where the singular value of $S(j \omega)$ becomes equal to one.

In case of a nonpassive macromodel, as per Theorem-2, the imaginary eigenvalues of the Hamiltonian matrix $\boldsymbol{M}$ of (4.7) provide the information about the frequency points where $\|S(j \omega)\|_{2}=1$. However, this information is not enough to perform the passivity enforcement. This is because, for passivity enforcement, the regions of passivity violation (i.e. the frequency bandwidth in which $\|S(j \omega)\|>1)$, should be known. Using the information of the imaginary eigenvalues of $\boldsymbol{M}$, the next section provides a systematic method for identifying the regions of passivity violation. 


\subsubsection{Third Step: Determination of Passivity Violating Regions [47], [50] - [53]}

In the proposed algorithm, the regions of passivity violation are determined by evaluating the slope of eigenvalues of $S(j \omega)^{H} S(j \omega)$ at frequency points corresponding to the imaginary eigenvalues of $\boldsymbol{M}$. This is done as follows (for the purpose of simplicity, from this point onwards $S(j \omega)^{H} S(j \omega)$ is denoted by $\tilde{\boldsymbol{S}}(j \omega))$.

If $\lambda$ is an eigenvalue of $\tilde{\boldsymbol{S}}(j \omega)$ and $\boldsymbol{u}$ the corresponding right eigenvector, then

$$
(\tilde{\boldsymbol{S}}(j \omega)-\lambda \boldsymbol{I}) \boldsymbol{u}=0
$$

Differentiating the above equation with respect to the angular frequency $\omega$ we get

$$
\left(\frac{d}{d \omega} \tilde{\boldsymbol{S}}(j \omega)-\frac{d \lambda}{d \omega} \boldsymbol{I}\right) \boldsymbol{u}+(\tilde{\boldsymbol{S}}(j \omega)-\lambda \boldsymbol{I}) \frac{d \boldsymbol{u}}{d \omega}=0
$$

Next, multiplying (4.35) by the left eigenvector $\left(v^{T}\right)$ of $\tilde{S}(j \omega)$, we get

$$
\boldsymbol{v}^{T} \frac{d}{d \omega} \tilde{\boldsymbol{S}}(j \omega) \boldsymbol{u}-\boldsymbol{v}^{T} \frac{d \lambda}{d \omega} \boldsymbol{u}+\boldsymbol{v}^{T} \underbrace{(\tilde{\boldsymbol{S}}(j \omega)-\lambda \boldsymbol{I})}_{=0} \frac{d \boldsymbol{u}}{d \omega}=0
$$

Notice that the last term on the left hand side of (4.36) is zero by the definition of left eigenvector. Using this fact, (4.36) can be rewritten as

$$
\boldsymbol{v}^{T} \frac{d \lambda}{d \omega} \boldsymbol{u}=\boldsymbol{v}^{T} \frac{d}{d \omega} \tilde{S}(j \omega) \boldsymbol{u}
$$

or, 


$$
\frac{d \lambda}{d \omega}=\frac{v^{T} \frac{d}{d \omega} \tilde{S}(j \omega) \boldsymbol{u}}{v^{T} \boldsymbol{u}}
$$

where,

$$
\begin{aligned}
\frac{d}{d \omega} \tilde{\boldsymbol{S}}(j \omega)= & {\left[-\boldsymbol{B}^{T}\left(-j \omega \boldsymbol{I}-\boldsymbol{A}^{T}\right)^{-2}(-j) \boldsymbol{C}^{T}\right]\left[\boldsymbol{C}(j \omega \boldsymbol{I}-\boldsymbol{A})^{-1} \boldsymbol{B}+\boldsymbol{D}\right] } \\
& +\left[\boldsymbol{B}^{T}\left(-j \omega \boldsymbol{I}-\boldsymbol{A}^{T}\right)^{-1} \boldsymbol{C}^{T}+\boldsymbol{D}^{T}\right]\left[-\boldsymbol{C}(j \omega \boldsymbol{I}-\boldsymbol{A})^{-2} j \boldsymbol{B}\right]
\end{aligned}
$$

and $v^{T}$ is the left eigenvector of $\tilde{S}(j \omega)$ corresponding to the eigenvalue $\lambda$. Using (4.38), the slope of eigenvalues of $\tilde{\boldsymbol{S}}(j \omega)$, at frequency points of passivity violation can be determined. The regions and bandwidth of local passivity violation are then determined using the following steps:

- Step 1: Collect the pure imaginary eigenvalues (consider only those with positive imaginary parts) of the Hamiltonian matrix $\boldsymbol{M}$ of (4.7) in a vector $S_{a}=\left[\omega_{1}, \omega_{2} \ldots \omega_{T}\right]$, such that $\omega_{1}<\omega_{2}<\ldots<\omega_{T}$, where ' $T$ ' is the total number of such entries. Let $\omega_{H}=\omega_{T}$.

- Step 2: Next, at the frequency corresponding to each of the above entries, evaluate the slope of the eigenvalue of $\tilde{\boldsymbol{S}}(j \omega)$ using (4.38). Note that the slope at $\omega_{T}$ is always negative since, $\|D\|_{2}<1$ is ensured during the fitting process.

- Step 3: Count the number of positive and negative slopes starting from $\omega_{H}$. When the count of positive and negative slopes become equal, say at $\omega_{k}$, then the first region of local passivity violation is established (i.e. the region between $\omega_{H}$ to $\left.\omega_{k}\right)$.

- Step 4: Reset the count of slope to zero and designate $\omega_{H}=\omega_{k-1}$ and repeat steps 3 and 4 until all entries in the vector $S_{a}$ are exhausted.

The above steps are illustrated in Fig. 4.3(a), for the case of Hamiltonian matrix having six pairs of imaginary eigenvalues. 
The situation when the passivity violation starts at zero frequency is illustrated with an example in Fig. 4.3(b). As seen in this example, when the counting of slopes is restarted after determining the second region of violation, the number of negative slopes becomes one and the number of positive slopes is equal to zero, at the end of counting the slopes. In such cases, a region of violation exists between the first frequency point from where the counting of slope is restarted (in this case $\omega_{1}$ ) and zero Hertz (origin).

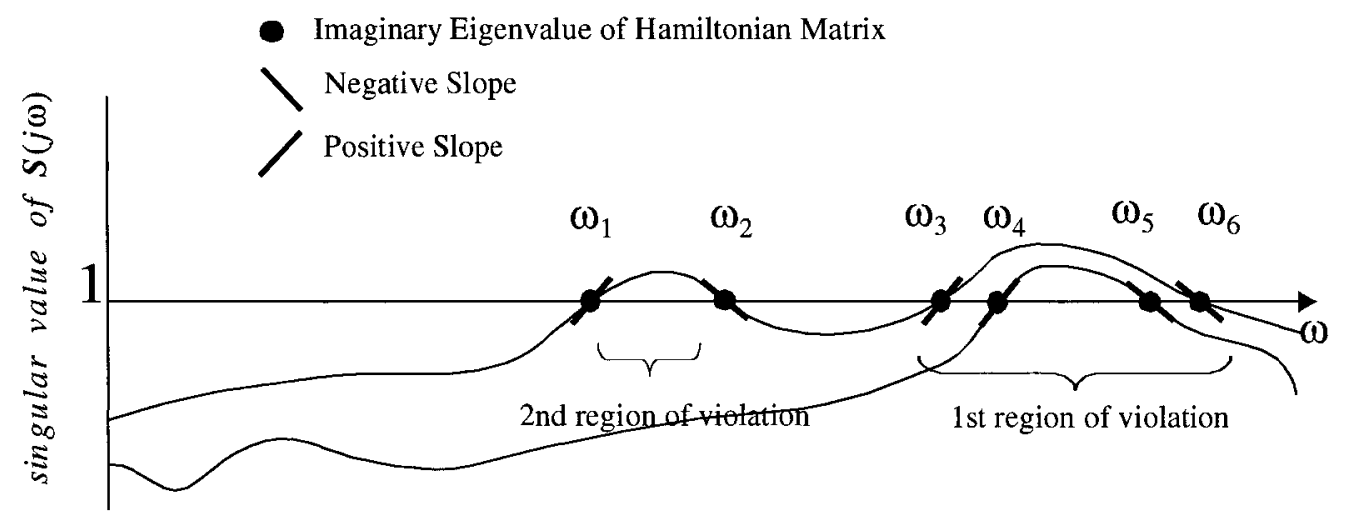

(a)

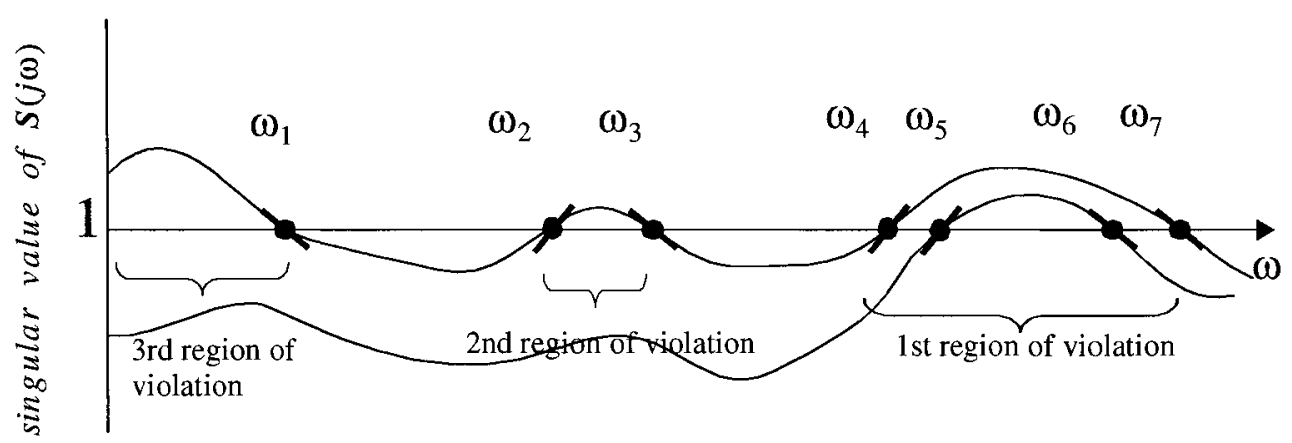

(b)

Fig. 4.3. Illustration of identification of regions of passivity violation 
Next, in each region of passivity violation, the location of maximum passivity violation is determined as per the procedure described in the next section.

\subsection{3a Determination of Location of Maximum Violation in a Non- Passive Region}

Having determined the regions of passivity violation, the exact location of maximum passivity violation (i.e. maximum eigenvalue of $\tilde{\boldsymbol{S}}(j \omega)$ ) is determined in each region of passivity violation (these locations are addressed first during the passivity enforcement process). One way to approximately (since the exact location is not very crucial for compensation) determine these locations is to perform a fine frequency sweep of $\|S(j \omega)\|_{2}$ versus frequency in each passivity violating region. Alternatively, they can be accurately determined by solving the following problem in each region of passivity violation.

$$
\max \operatorname{eig}(\tilde{\boldsymbol{S}}(j \omega)) ; \quad \omega_{l} \leq \omega \leq \omega_{h}
$$

$\omega$

(4.40)

where $\omega_{l}, \omega_{h}$ are the boundaries of a passivity violating region. For instance, in the first region of violation in Fig. 4.3(a), $\omega_{l}=\omega_{3}$ and $\omega_{h}=\omega_{6}$. The problem in (4.40) converges very fast as it is associated with only one variable $(\omega)$ and has a good initial guess (midpoint of $\omega_{l}$ and $\omega_{h}$ ).

Based on the above information about the locations of maximum violation, the proposed passivity enforcement algorithm is described in the next section. 


\subsubsection{Fourth Step: Passivity Enforcement}

With the information about passivity violation locations from Section 4.2.3, the macromodel is compensated for any passivity violation. For this purpose, this section presents a fast passivity compensation algorithm, with two different implementation techniques. The first implementation is based on iterative firstorder perturbation of residues, while the second implementation is based on the linear matrix inequality (LMI) formulation. In order to minimize the error introduced in the response of the macromodel during compensation process, a cost function is added as a constraint during passivity compensation.

The starting point of both the passivity compensation techniques is the macromodel obtained from the first step (Section 4.2.1). It is assumed that the macromodel s poles are sufficient to approximate the original data with desired accuracy. Given this, the passivity compensation algorithms described in this section perturb the residue matrix $\boldsymbol{C}$ such that the resulting macromodel is passive and also satisfies the accuracy constraints of the approximation.

\subsection{4a Compensation by First-Order Perturbation (PCFOP)}

In this algorithm, the passivity compensation is carried out by perturbing the residue matrix $C$ so as to compensate for the passivity violation. The algorithm is iterative in nature, with small perturbations applied to the matrix $C$ in each iteration and correcting the passivity violation in increments. Fig. 4.4 illustrates the main idea behind the algorithm. 


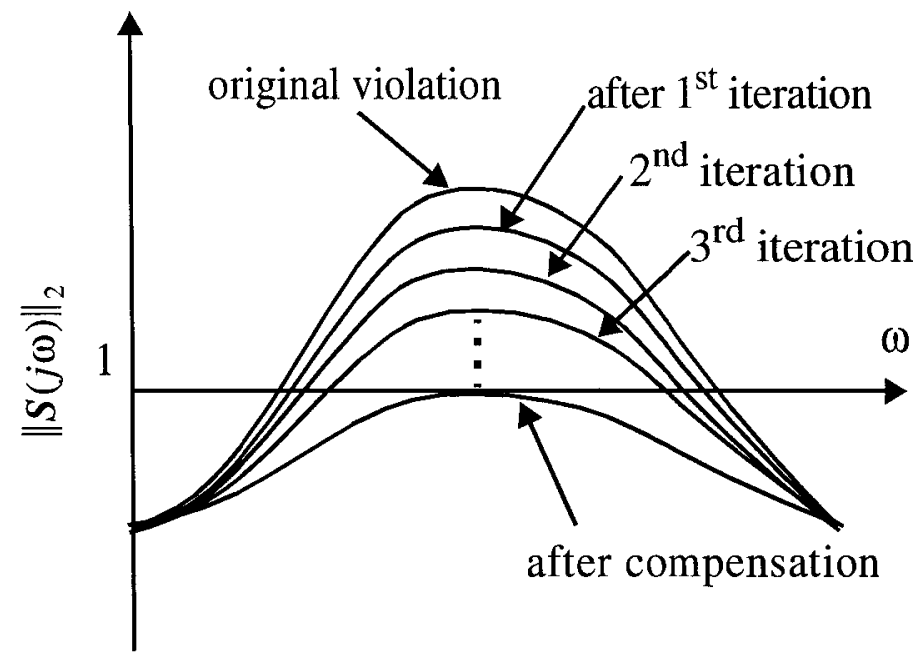

Fig. 4.4. Passivity compensation by perturbation of matrix $C$

It can be seen from Fig. 4.4 that the passivity violation is gradually reduced in each iteration, until $\|S(j \omega)\|_{2} \leq 1$ over the entire passivity violating region. With a small perturbation $\Delta C$ in matrix $C$, the perturbed scattering transfer matrix represented by $\hat{\boldsymbol{S}}(j \omega)$ can be expressed as

$$
\hat{\boldsymbol{S}}(j \omega)=(\boldsymbol{C}+\Delta \boldsymbol{C})(j \omega \boldsymbol{I}-\boldsymbol{A})^{-1} \boldsymbol{B}+\boldsymbol{D}
$$

or

$$
\hat{S}(j \omega)=S(j \omega)+\Delta S(j \omega)
$$

where

$$
\Delta S(j \omega)=\Delta C(j \omega I-A)^{-1} B
$$

Condition (c) for passivity (defined in Section 4.1) states that (with conditions (a) and (b) satisfied), a network is passive if its scattering matrix $S(j \omega)$ satisfies

$$
\boldsymbol{I}-\boldsymbol{S}(j \omega)^{H} \boldsymbol{S}(j \omega) \geq \mathbf{0}
$$


In the proposed algorithm, if the macromodel of (4.5) is non-passive (i.e. $\boldsymbol{I}-\boldsymbol{S}(j \omega)^{H} \boldsymbol{S}(j \omega)$ is negative definite), then the matrix $\boldsymbol{C}$ is perturbed by $\Delta \boldsymbol{C}$ such that the new model $\hat{S}(j \omega)=S(j \omega)+\Delta S(j \omega)$ is passive at the frequency points of violation. The matrices $\boldsymbol{A}, \boldsymbol{B}$ and $\boldsymbol{D}$ are kept fixed during this process. The desired perturbation $\Delta \boldsymbol{C}$ is calculated using the first-order eigenvalue perturbation formulae [82], according to which, if a matrix $\boldsymbol{K}$ is perturbed by an amount $\Delta \boldsymbol{K}$, then the resulting change $\Delta \lambda$, in its eigenvalue is given by

$$
\Delta \lambda=\frac{\boldsymbol{y}^{T} \Delta \boldsymbol{K} \boldsymbol{x}}{\boldsymbol{y}^{T} \boldsymbol{x}}
$$

where $\boldsymbol{y}$ and $\boldsymbol{x}$ are the left and right eigenvectors respectively, of $\boldsymbol{K}$.

The objective here is that, the perturbed model $\hat{S}(j \omega)=\boldsymbol{S}(j \omega)+\Delta \boldsymbol{S}(j \omega)$ satisfies the following condition at the frequency points of violation

$$
\boldsymbol{I}-\hat{\boldsymbol{S}}(j \omega)^{H} \hat{\boldsymbol{S}}(j \omega) \geq \mathbf{0}
$$

or, substituting $\hat{S}(j \omega)=S(j \omega)+\Delta S(j \omega)$ (for simplicity, dropping $j \omega)$

$$
\boldsymbol{I}-\boldsymbol{S}^{H} \boldsymbol{S}-\boldsymbol{S}^{H} \Delta \boldsymbol{S}-\Delta \boldsymbol{S}^{H} \boldsymbol{S}-\Delta \boldsymbol{S}^{H} \Delta \boldsymbol{S} \geq \mathbf{0}
$$

Neglecting the second-order term in the perturbation $\Delta S$, we have

$$
\boldsymbol{I}-\boldsymbol{S}^{H} \boldsymbol{S}-\boldsymbol{S}^{H} \Delta \boldsymbol{S}-\Delta \boldsymbol{S}^{H} \boldsymbol{S} \geq \mathbf{0}
$$

Equation (4.48) implies that, if the original model is non-passive, i.e. an eigenvalue of $\boldsymbol{I}-\boldsymbol{S}^{H} \boldsymbol{S}$ is negative by an amount $\Delta \lambda$, then perturbing $\boldsymbol{I}-\boldsymbol{S}^{H} \boldsymbol{S}$ by $-\boldsymbol{S}^{H} \Delta \boldsymbol{S}-\Delta \boldsymbol{S}^{H} \boldsymbol{S}$, such that 


$$
\Delta \lambda=\frac{\boldsymbol{v}^{T}\left(-\boldsymbol{S}^{H} \Delta \boldsymbol{S}-\Delta \boldsymbol{S}^{H} \boldsymbol{S}\right) \boldsymbol{u}}{\boldsymbol{v}^{T} \boldsymbol{u}}
$$

will cancel $-\Delta \lambda$ from the corresponding negative eigenvalue of $\boldsymbol{I}-\boldsymbol{S}^{H} \boldsymbol{S}$. Here $\boldsymbol{v}$ and $\boldsymbol{u}$ are the left and right eigenvectors of $\boldsymbol{I}-\boldsymbol{S}^{H} \boldsymbol{S}$ respectively.

Substituting for $\boldsymbol{S}$ from (4.5) and $\Delta S$ from (4.43) and noting that $v^{T} u=1$, (4.49) can be written as

$$
\begin{aligned}
\Delta \lambda= & -\boldsymbol{v}^{T}\left\{\left[\boldsymbol{D}^{T} \Delta \boldsymbol{C}(s \boldsymbol{I}-\boldsymbol{A})^{-1} \boldsymbol{B}\right]+\left[\boldsymbol{B}^{T}\left(s^{H} \boldsymbol{I}-\boldsymbol{A}^{T}\right)^{-1} \boldsymbol{C}^{T} \Delta \boldsymbol{C}(s \boldsymbol{I}-\boldsymbol{A})^{-1} \boldsymbol{B}\right]+\ldots\right. \\
& \left.\ldots\left[\boldsymbol{B}^{T}\left(s^{H} \boldsymbol{I}-\boldsymbol{A}^{T}\right)^{-1} \Delta \boldsymbol{C}^{T} \boldsymbol{C}(s \boldsymbol{I}-\boldsymbol{A})^{-1} \boldsymbol{B}\right]+\left[\boldsymbol{B}^{T}\left(s^{H} \boldsymbol{I}-\boldsymbol{A}^{T}\right)^{-1} \Delta \boldsymbol{C}^{T} \boldsymbol{D}\right]\right\} \boldsymbol{u}
\end{aligned}
$$

Using the property of Kronecker products [79], (4.50) can be expressed as

$$
\begin{aligned}
\Delta \lambda & =-\left\{\left[\left((s \boldsymbol{I}-\boldsymbol{A})^{-1} \boldsymbol{B} \boldsymbol{u}\right)^{T} \otimes \boldsymbol{v}^{T} \boldsymbol{D}^{T}\right]+\left[\left((s \boldsymbol{I}-\boldsymbol{A})^{-1} \boldsymbol{B} \boldsymbol{u}\right)^{T} \otimes \boldsymbol{B}^{T}\left(s^{H} \boldsymbol{I}-\boldsymbol{A}^{T}\right)^{-1} \boldsymbol{C}^{T}\right]+\ldots\right. \\
& \left.\ldots\left[\left((s \boldsymbol{I}-\boldsymbol{A})^{-1} \boldsymbol{B} \boldsymbol{v}\right)^{T} \otimes \boldsymbol{u}^{T} \boldsymbol{B}^{T}\left(s^{H} \boldsymbol{I}-\boldsymbol{A}^{T}\right)^{-1} \boldsymbol{C}^{T}\right]+\left[\left((s \boldsymbol{I}-\boldsymbol{A})^{-1} \boldsymbol{B} \boldsymbol{v}\right)^{T} \otimes \boldsymbol{u}^{T} \boldsymbol{D}^{T}\right]\right\} \operatorname{vec}(\Delta \boldsymbol{C})
\end{aligned}
$$

where, $\otimes$ represents the Kronecker product operator and $\operatorname{vec}(\Delta C)$ represents the rows of $\Delta \boldsymbol{C}$ stacked in vector format. Equation (4.51) can be more compactly written as

$$
W \boldsymbol{X}=\Delta \lambda
$$

where $X(\operatorname{vec}(\Delta C))$ is the vector of unknowns of the perturbation matrix $\Delta C$, while $\boldsymbol{W}$ is composed of entries of matrix $\boldsymbol{A}, \boldsymbol{B}, \boldsymbol{C}, \boldsymbol{D}$ and $\omega$ (it represents the term between parentheses \{\} on the RHS of (4.51)). The problem in (4.52) is solved iteratively at the frequency points of maximum violation in each region of the passivity violation, with the constraint that the error in the response is minimized. 
With the perturbation $\Delta C$ in the residue matrix $C, L_{2}$ norm of error in the response $\Delta \boldsymbol{S}(j \omega)$ is given by [81]

$$
\begin{array}{r}
\|\Delta S\|_{2}^{2}=\int_{-\infty}^{\infty}\|\Delta S(j \omega)\|_{F}^{2} d \omega=\int_{-\infty i=1}^{\infty} \sum_{k=1}^{m} \sum_{k}^{m}\left|\Delta S_{i k}(j \omega)\right|^{2} d \omega=\int_{-\infty i=1}^{\infty} \sum_{k=1}^{m} \sum_{i k}^{m}\left|\Delta S_{i k}(t)\right|^{2} d t=\ldots \\
\ldots=\operatorname{trace}\left(\Delta \boldsymbol{C} \boldsymbol{P} \Delta \boldsymbol{C}^{T}\right)
\end{array}
$$

where $\|\Delta S(j \omega)\|_{F}$ is the Frobenius norm of $\Delta S(j \omega)$ and $m$ is the number of ports. $\boldsymbol{P}$ is the controllability Grammian obtained by solving the following Lyapunov equation

$$
\boldsymbol{A P}+\boldsymbol{P} \boldsymbol{A}^{H}+\boldsymbol{B} \boldsymbol{B}^{H}=\mathbf{0}
$$

In effect, the following problem is solved iteratively at the frequency points of maximum violation, to satisfy the passivity criteria while keeping the error in the response to the minimum:

$$
\begin{gathered}
\min \left(\operatorname{trace}\left(\Delta \boldsymbol{C} \boldsymbol{P} \Delta \boldsymbol{C}^{T}\right)\right) ; \quad \text { such that, } \\
\Delta \boldsymbol{C} \\
\quad \boldsymbol{W X}=\Delta \lambda
\end{gathered}
$$

where $\boldsymbol{X}$ consists of the elements of $\Delta \boldsymbol{C}$ arranged in a column. During an iteration, $W$ consists of the information of all the frequency points where the passivity correction is desired. As such $\boldsymbol{W} \in \mathfrak{R}^{n_{f} \times q m}$, with $n_{f}$ representing the number of frequency points where the passivity correction is being done and $q=n m$ is the order of the system, with $n$ the number of poles in the common pole-set. For implementation purposes, the objective function in (4.55) can be further modified as follows 


$$
\begin{gathered}
\operatorname{trace}\left(\Delta \boldsymbol{C} \quad \boldsymbol{P} \quad \Delta \boldsymbol{C}^{T}\right)=\boldsymbol{X}^{T} \boldsymbol{H} \boldsymbol{X} ; \\
\boldsymbol{H}=\left[\begin{array}{cccc}
\boldsymbol{P} & 0 & \ldots & 0 \\
0 & \boldsymbol{P} & \ldots & 0 \\
\vdots & \vdots & \ldots & \vdots \\
0 & 0 & \ldots & \boldsymbol{P}
\end{array}\right]_{(q m) \times(q m)}
\end{gathered}
$$

Using (4.56), (4.55) can be written as

$$
\begin{gathered}
\min \left(\boldsymbol{X}^{T} \boldsymbol{H} \boldsymbol{X}\right) ; \quad \text { such that }, \\
\Delta \boldsymbol{C} \\
\boldsymbol{W X}=\Delta \lambda
\end{gathered}
$$

It is evident that the objective function in (4.57) is quadratic in nature and therefore can be solved using efficient routines directed specifically towards minimizing the quadratic function [47], [50].

It is to be noted that, during each iteration, passivity correction is accomplished by perturbing only the residues. The overall residue matrix after compensating all the regions of violation is obtained by updating the residue matrix $C$ with the perturbation $\Delta C$ after compensating each region. For instance, once the required perturbation $\Delta C_{1}$ is computed for the first region, the residue matrix $C$ is updated as $C_{1}=C+\Delta C_{1}$. When the compensation is performed for the next region of violation, new residue matrix $C_{1}$ is updated by $\Delta C_{2}$ for the required compensation as $C_{2}=C_{1}+\Delta C_{2}$. This process is continued for all the regions of violation and the resulting updated matrix $C_{g}$ (for the case of $g$ regions of violation) becomes the final residue matrix for the passive macromodel. 


\subsection{4b Compensation by Linear Matrix Inequality (PCLMI)}

In this section, an alternative implementation for the proposed passivity compensation by formulating (4.47) in terms of linear matrix inequality (LMI) [47], [50] is described. Neglecting the second order term in the perturbation $\Delta \boldsymbol{S}$, (4.47) can be written in the form of LMI as

$$
\boldsymbol{I}-\boldsymbol{S}^{H} \boldsymbol{S}-\boldsymbol{S}^{H} \Delta \boldsymbol{S}-\Delta \boldsymbol{S}^{H} \boldsymbol{S} \geq \mathbf{0}
$$

where, the matrix on the L.H.S. of (4.58) is complex-valued and Hermitian.

Almost all the available LMI solvers are written for real-valued matrices and may not directly handle LMI problems involving complex-valued matrices. However, complex-valued LMIs can be converted into real-valued LMIs by using the following lemma:

Lemma 1: A complex Hermitian matrix $\boldsymbol{S}$ satisfies

$$
S>0
$$

iff

$$
\left[\begin{array}{cc}
\operatorname{Re}(\boldsymbol{S}) & \operatorname{Im}(\boldsymbol{S}) \\
-\operatorname{Im}(\boldsymbol{S}) & \operatorname{Re}(\boldsymbol{S})
\end{array}\right]>0
$$

Proof of Lemma 1: For the proof, it is important to note that a complex Hermitian matrix satisfies the following properties:

a) The imaginary part of the diagonal elements is zero

b) The real part of the Hermitian matrix is symmetric

c) The imaginary part of a Hermitian matrix is Hermitian. 
Separating real $\left(\boldsymbol{S}_{R}\right)$ and imaginary $\left(\boldsymbol{S}_{I}\right)$ parts, $\boldsymbol{S}$ can be written as

$$
S=S_{R}+j S_{I}
$$

Equation (4.59) then implies that for any arbitrary vector $z$

$$
z^{H}\left[S_{R}+j S_{I}\right] z>0
$$

Substituting $z=z_{1}+j z_{2}$ and expanding (4.62), we get

$$
\begin{aligned}
& z_{1}^{T} S_{R} z_{1}+z_{2}^{T} S_{I} z_{1}-z_{1}^{T} S_{1} z_{2}+z_{2}^{T} S_{R} z_{2}+\ldots \\
& \quad+j\left(z_{1}^{T} S_{I} z_{1}-z_{2}^{T} S_{R} z_{1}+z_{1}^{T} S_{R} z_{2}+z_{2}^{T} S_{I} z_{2}\right)>0
\end{aligned}
$$

In view of the above indicated property (b) of the Hermitian matrix, it is evident that

$$
z_{1}^{T} S_{R} z_{2}=z_{2}^{T} S_{R} z_{1} ; \quad \forall z_{1}, z_{2}
$$

Also, using properties (a) and (c) of the Hermitian matrix, we have

$$
\boldsymbol{z}_{1}^{T} \boldsymbol{S}_{I} \boldsymbol{z}_{1}=0 ; \quad \boldsymbol{z}_{2}^{T} \boldsymbol{S}_{I} \boldsymbol{z}_{2}=0
$$

Using (4.64) and (4.65), it can be seen that the imaginary part of (4.63) is zero. As a result, (4.63) can be written as

$$
z_{1}^{T} S_{R} z_{1}+z_{2}^{T} S_{1} z_{1}-z_{1}^{T} S_{1} z_{2}+z_{2}^{T} S_{R} z_{2}>0
$$

or

$$
\left[\begin{array}{ll}
z_{2}^{T} & z_{1}^{T}
\end{array}\right]\left[\begin{array}{cc}
S_{R} & S_{I} \\
-S_{I} & S_{R}
\end{array}\right]\left[\begin{array}{l}
z_{2} \\
z_{1}
\end{array}\right]>0
$$

Equation (4.67) implies that 


$$
\left[\begin{array}{cc}
S_{R} & S_{I} \\
-S_{I} & S_{R}
\end{array}\right]>0
$$

This proves the Lemma 1. Next, expressing the scattering matrix as

$$
S=S_{R}+j S_{I}
$$

and

$$
\Delta \boldsymbol{S}=\Delta \boldsymbol{S}_{R}+j \Delta \boldsymbol{S}_{I}
$$

and using Lemma 1, (4.58) can be written as

$$
\left[\begin{array}{cc}
\boldsymbol{S}_{R} & \boldsymbol{S}_{I} \\
-\boldsymbol{S}_{I} & \boldsymbol{S}_{R}
\end{array}\right]^{T}\left[\begin{array}{cc}
\boldsymbol{S}_{R} & \boldsymbol{S}_{I} \\
-\boldsymbol{S}_{I} & \boldsymbol{S}_{R}
\end{array}\right]+\left[\begin{array}{cc}
\boldsymbol{S}_{R} & \boldsymbol{S}_{I} \\
-\boldsymbol{S}_{I} & \boldsymbol{S}_{R}
\end{array}\right]^{T}\left[\begin{array}{cc}
\Delta \boldsymbol{S}_{R} & \Delta \boldsymbol{S}_{I} \\
-\Delta \boldsymbol{S}_{I} & \Delta \boldsymbol{S}_{R}
\end{array}\right]+\left[\begin{array}{cc}
\Delta \boldsymbol{S}_{R} & \Delta \boldsymbol{S}_{I} \\
-\Delta \boldsymbol{S}_{I} & \Delta \boldsymbol{S}_{R}
\end{array}\right]^{T}\left[\begin{array}{ll}
\boldsymbol{S}_{R} & \boldsymbol{S}_{I} \\
-\boldsymbol{S}_{I} & \boldsymbol{S}_{R}
\end{array}\right]<\boldsymbol{I}
$$

The LMI (4.71) is solved subjected to the constraint that the error in the response (4.56) is minimized.

The two techniques presented above (in Section 4.2.4a and Section 4.2.4b) are different implementations of the same problem defined in (4.47). It has been found that the performance of the two techniques are comparable. If a good LMI solver is readily available, then PCLMI technique may be easier to implement as there is no need to keep track of the extent of the first-order perturbation required during each iteration (since the desired perturbation $\Delta \boldsymbol{C}$ is calculated in one go at a frequency point of violation).

A good measure of the effect of the required perturbation for passivity enforcement on the macromodel response is governed by the amount of change $(\Delta C)$ made to the residue matrix $C$. The passivity compensation formulation ((4.57) or (4.71)), perturbs the macromodel such that the error in the response 
(which is a function of $\Delta C$ ) is minimized. There can be two possibilities, when the relative perturbation in the residue matrix $\|\Delta C\|_{F} /\|C\|_{F}$ is large for compensating the macromodel: either the original data is nonpassive by a large amount or the approximation of the data was not good enough.

A summary of the steps involved in the proposed passivity enforcement algorithm is given in Fig. 4.5. 
- Step 1: Obtain multiport tabulated data up to $f_{\text {max }}$ (maximum frequency of interest).

- Step 2: Compute the multiport pole-residue model (4.3). Obtain the state-space system $(\boldsymbol{A}, \boldsymbol{B}, \boldsymbol{C}, \boldsymbol{D})(4.4)$.

- Step 3: Construct the Hamiltonian matrix (4.7) and check its eigenvalues.

if imaginary eigenvalues are found, macromodel is nonpassive.

- Step 4: Collect the pure imaginary eigenvalues (consider only those with positive imaginary parts) of the Hamiltonian matrix $M$ of (4.7) in a vector $S_{a}=\left[\omega_{1}, \omega_{2} \ldots \omega_{T}\right]$, such that $\omega_{1}<\omega_{2}<\ldots<\omega_{T}$, where ' $T$ ' is the total number of such entries. Let $\omega_{H}=\omega_{T}$.

- Step 5: Determine the regions of violation using the following steps as outlined in Section 4.2.3:

(i) At the frequency corresponding to each of the entries in the vector $S_{a}$, evaluate the slope of the eigenvalue of $\tilde{\boldsymbol{S}}(j \omega)$ using (4.38).

(ii) Count the number of positive and negative slopes starting from $\omega_{H}$. When the count of positive and negative slopes become equal, say at $\omega_{k}$, then the first region of local passivity violation is established (i.e. the region between $\omega_{H}$ to $\omega_{k}$ ).

(iii) Reset the count of slope to zero and designate $\omega_{H}=\omega_{k-1}$ and repeat steps (ii) and (iii) until all entries in the set $\boldsymbol{S}_{a}$ are exhausted.

- Step 6: Determine the exact location of maximum passivity violation in every region of violation using (4.40).

- Step 7: Perform the passivity compensation, by solving (4.47) at the frequency points of maximum violation, subjected to the constraint (4.53). Obtain the updated residue matrix $C$. Go to Step 3.

Else stop.

Fig. 4.5. Pseudocode of the proposed passive macromodeling algorithm 
Once the passivity of the macromodel has been ensured, it can be linked to a circuit simulator for performing transient analysis.

It is worth mentioning that, during the development of this thesis an alternative algorithm was being developed [43] for the passivity enforcement of tabulated macromodels. In this algorithm, the passivity is enforced by directly perturbing the Hamiltonian matrix of (4.7) such that the imaginary eigenvalues are shifted away from the imaginary $(j \omega)$ axis. A summary of this algorithm is given in Appendix B. It is to be noted that, compared to the technique in [43], the algorithm developed in thesis provides significant computational advantages. In [43], to determine the passivity violation region as well as to enforce the passivity via perturbation of eigenvalues of the Hamiltonian matrix, the eigenvectors of the Hamiltonian matrix [which is of size (2 x Num_of_Ports $\mathrm{x}$ Num_of_Poles)] were used. On the other hand, the proposed algorithm accomplishes the above using the eigenvectors of the transfer-function matrix which is of size (Num_of_Ports $x$ Num_of_Ports) much smaller than that of the Hamiltonian matrix, and hence leads to additional CPU savings in each iteration. Also, since the algorithm in [43] requires shifting of the imaginary eigenvalues, it needs their exact location for enforcing passivity. Unlike this, the proposed algorithm does not strictly require the exact information of imaginary eigenvalues. It can work even with the rough estimate of the regions of violation. This is a very useful feature of the proposed algorithm which can be exploited to save the computation time spent in evaluating the eigenvalues of Hamiltonian matrix in every iteration. In fact, this feature has further led to the development of computationally efficient passivity enforcement algorithm for large sized macromodels, which will be described in details in the next chapter. 


\subsection{Numerical Results}

In this section, several numerical examples are presented to validate the effectiveness of the proposed algorithm. Example 1 was obtained from [24], while example 2, 3, 4 and 5 are the test cases of industrial relevance, and were provided by Computer Simulation Technology (CST) GmBH, Germany. The CPU times reported here correspond to a PC platform with 1GB RAM and $2 \mathrm{GHz}$ AMD processor.

\subsubsection{Example 1: Three Port Transmission Line Network}

In this example, a 3-port distributed subnetwork [24], characterized by $S$ parameters (data is given up to $6 \mathrm{GHz}$ ) is considered. As per the proposed algorithm, in the first step, the data was modeled with a rational function approximation described in Section 4.2.1, using a common set of 44 poles. This resulted in a state-space system of order 132 (order = Number of ports $\mathrm{x}$ Number of poles). On checking the passivity using Theorem 1 of the second step (Section 4.2.2), it was found that the Hamiltonian matrix had three pairs of imaginary eigenvalues (meaning that model is nonpassive). A complete eigenvalue distribution of the associated Hamiltonian matrix is given in Fig. 4.6a. For the purpose of clarity, an enlarged view of the eigenvalue spread near the imaginary axis showing the numerical values of the imaginary eigenvalues is given in Fig. 4.6b. As per Theorem 2 (Section 4.2.2) these imaginary eigenvalues correspond to the location where the norm of $\boldsymbol{S}(j \omega)$ becomes one. This is illustrated in Fig. 4.6c, where it can be seen that the norm of $S(j \omega)$ becomes one at six frequency points corresponding to the imaginary eigenvalues of the Hamiltonian matrix. 


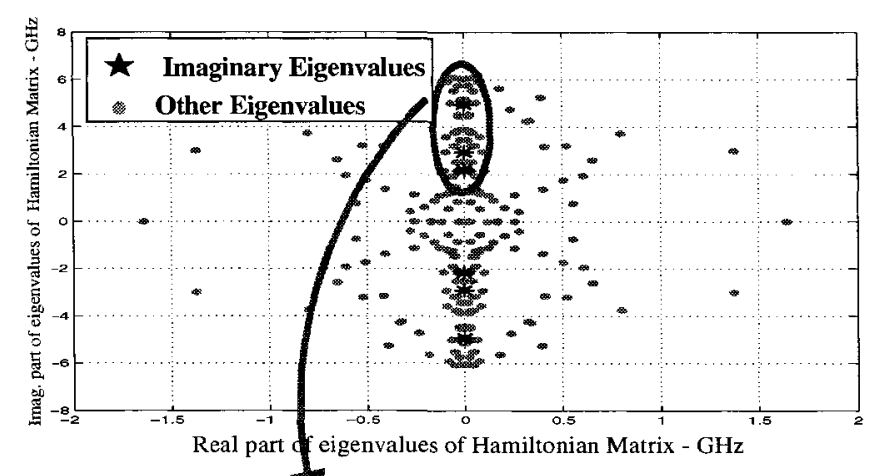

(a)

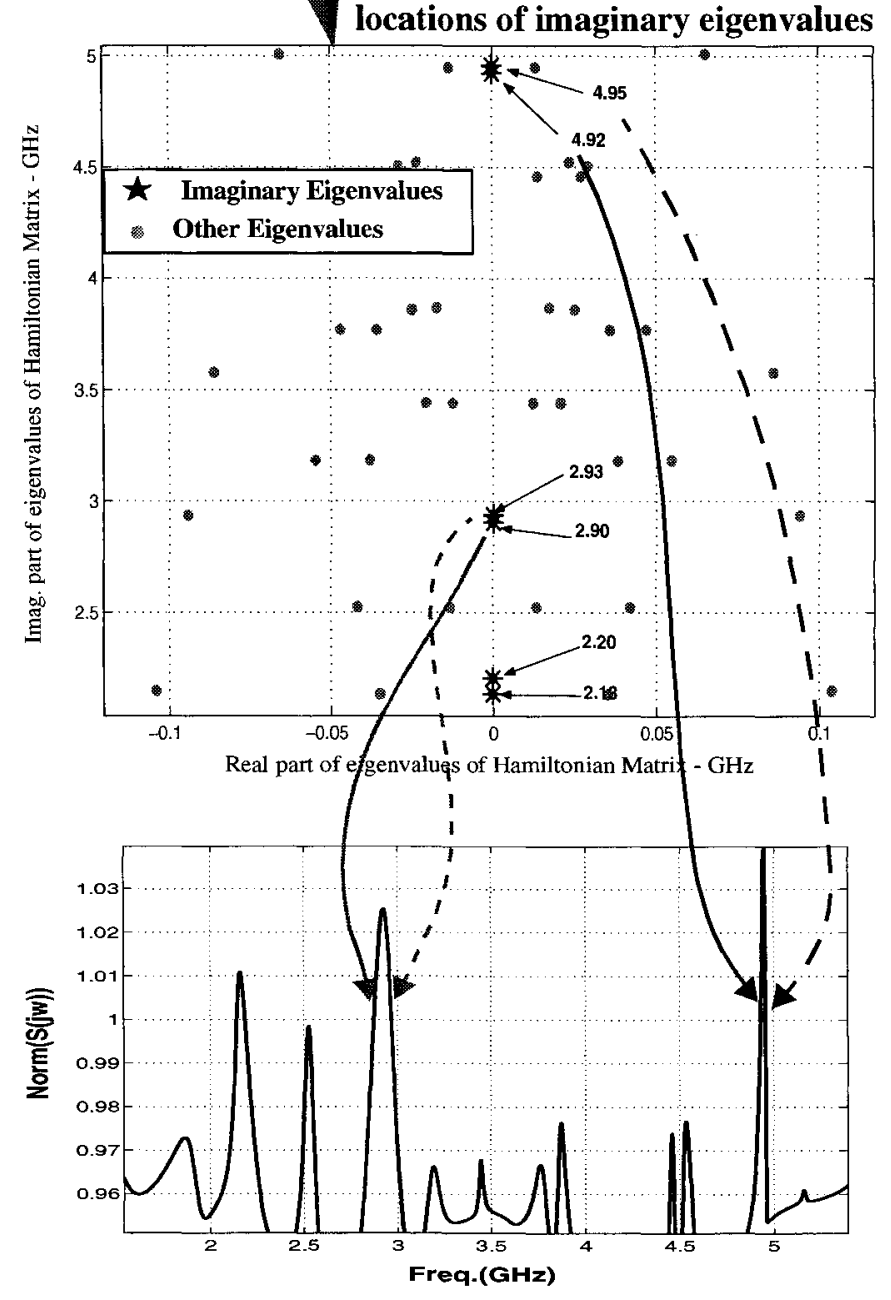

(b)

Fig. 4.6. Eigenvalue spectrum of Hamiltonian matrix 
Next, with this information, the passivity violation was quantified using third step (Section 4.2.3) and passivity compensation was performed using the algorithm described in Section 4.2.2 (fourth step). Fig. 4.7 shows a comparison of the 2-norm of the scattering matrix versus frequency of the original data and macromodel before and after compensation. The relative norm of the perturbation of the residue matrix $\|\Delta C\| /\|C\|$ for this example was $2.7 \times 10^{-3}$. The $S$-parameter response of the macromodel before and compensation is shown in Fig. 4.8. Fig. 4.9 shows the comparison of macromodel transient response (in the presence of nonlinear terminations) with the SPICE simulation of the original circuit, which match accurately.

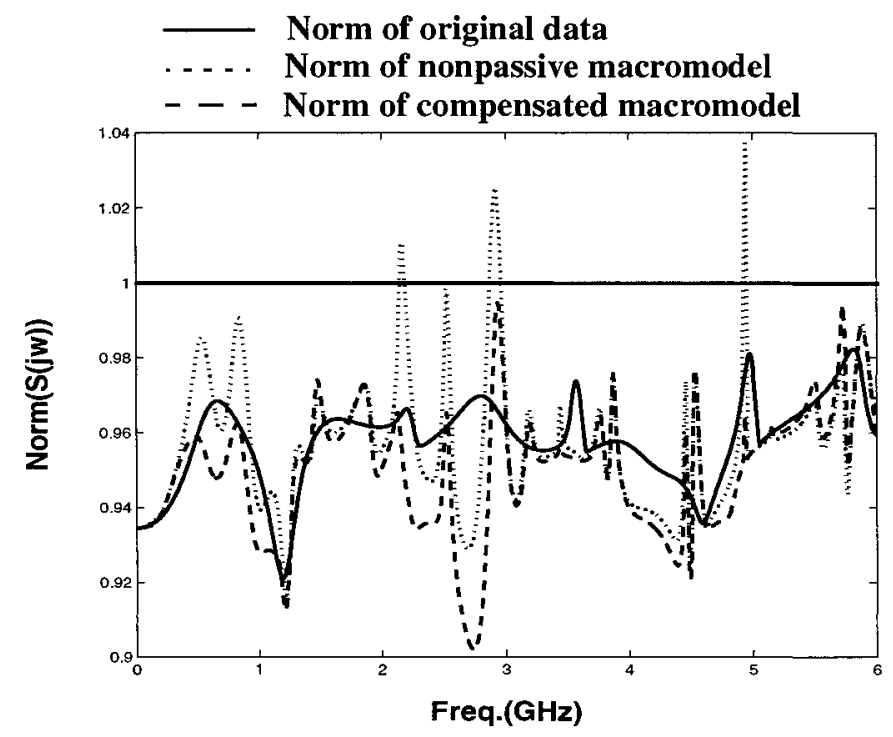

Fig. 4.7. Comparison of norm of $S(j \omega)$ : Three port network 


\section{- Original data \\ . . . . Response before compensation \\ - - - Response after compensation}
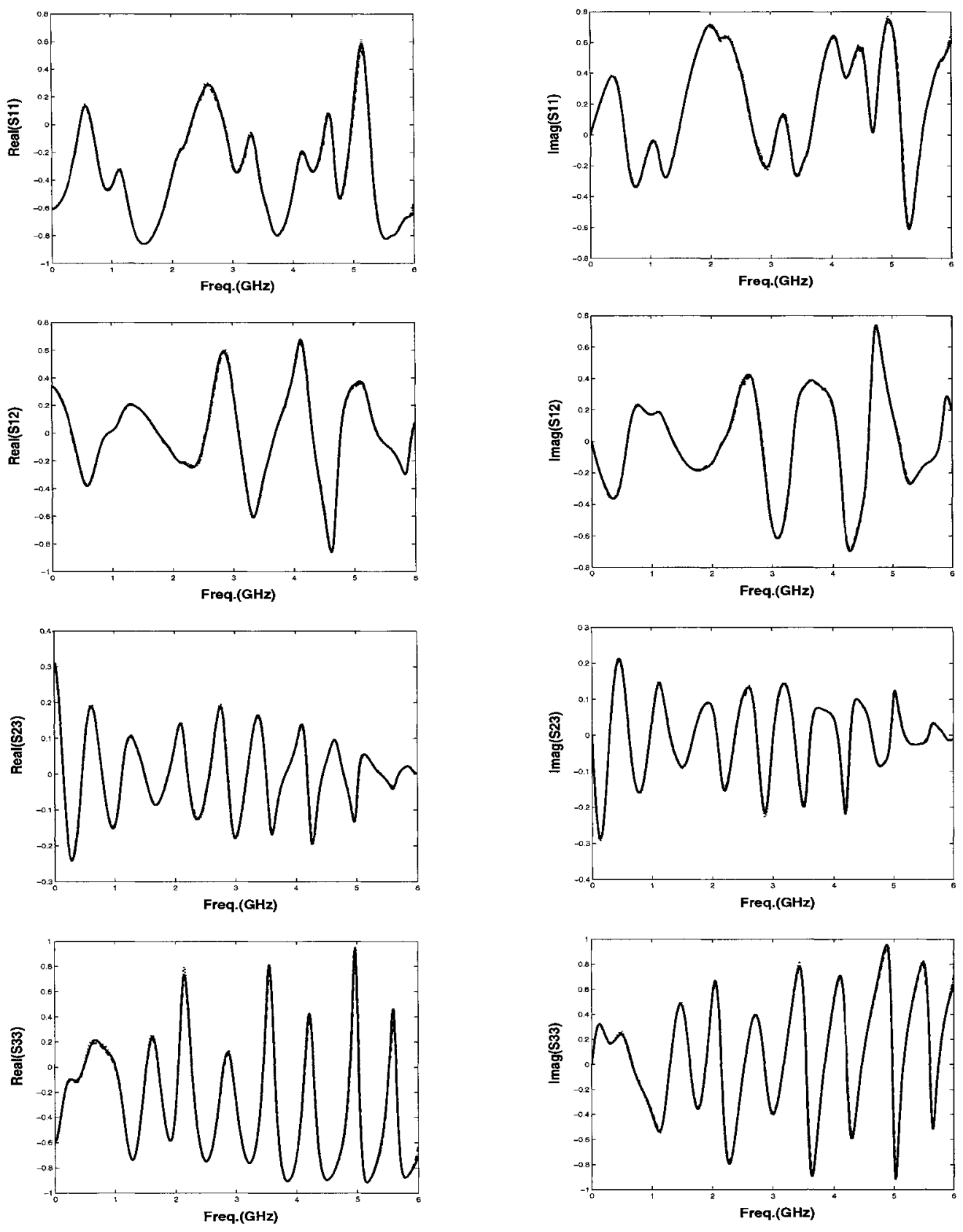

Fig. 4.8. S-parameter responses: Three port network 


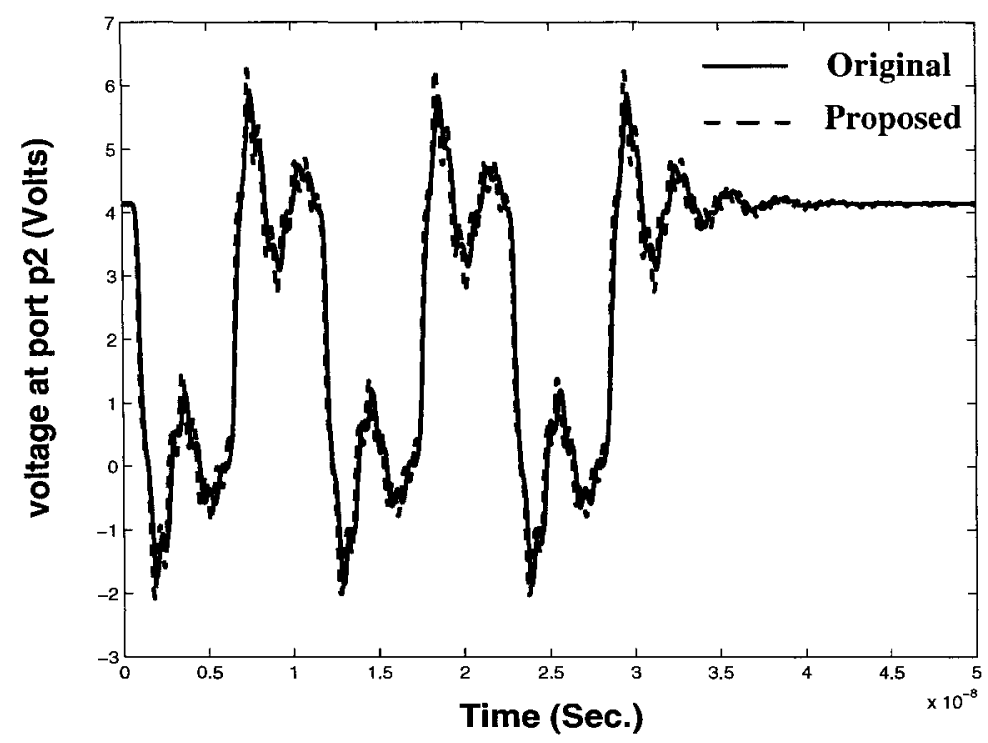

Fig. 4.9. Transient response: Three port network

\subsubsection{Example 2: Coaxial to Microstrip Coupler}

In this example, the tabulated $S$-parameter data of a 2-port coaxial-to-microstrip coupler up to $9 \mathrm{GHz}$ was considered. As per the proposed algorithm, in the first step, the data was modeled with a rational function approximation described in Section 4.2.1, using a common set of 30 poles. This resulted in a state-space system of order 60 . The resulting macromodel was found to be non-passive and was compensated using the proposed passivity checking and enforcement algorithm (described in Section 4.2.2, Section 4.2.3 and Section 4.2.4, which are the step 2, 3 and 4 of the proposed algorithm, respectively). It took 1 minute and 17 seconds for the entire passive macromodeling process. Fig. 4.10 shows a comparison of the 2-norm of the scattering matrix versus frequency of the original data and macromodel before and after compensation. The $S$-parameter response of the 
macromodel before and compensation is shown in Fig. 4.11. The relative norm of the perturbation of the residue matrix $\|\Delta C\| /\|C\|$ for this example was $5 \times 10^{-3}$.

It should be noted that there was no significant difference in CPU time required for passivity compensation when the PCFOP was replaced by the compensation algorithm based on linear matrix inequality (PCLMI - Section 4.2.4b) during the above analysis.

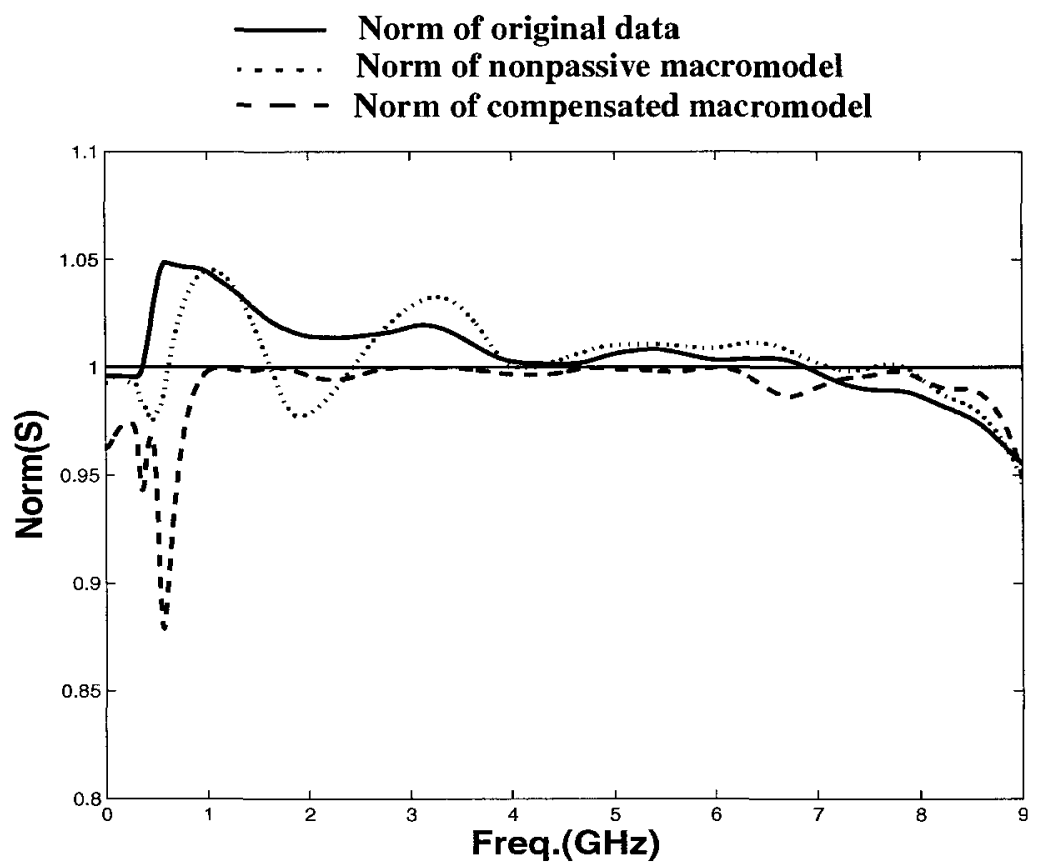

Fig. 4.10. Comparison of norm of $S(j \omega)$ : Coaxial to microstrip coupler 
- Original data

. . . . Response before compensation

- - - Response after compensation
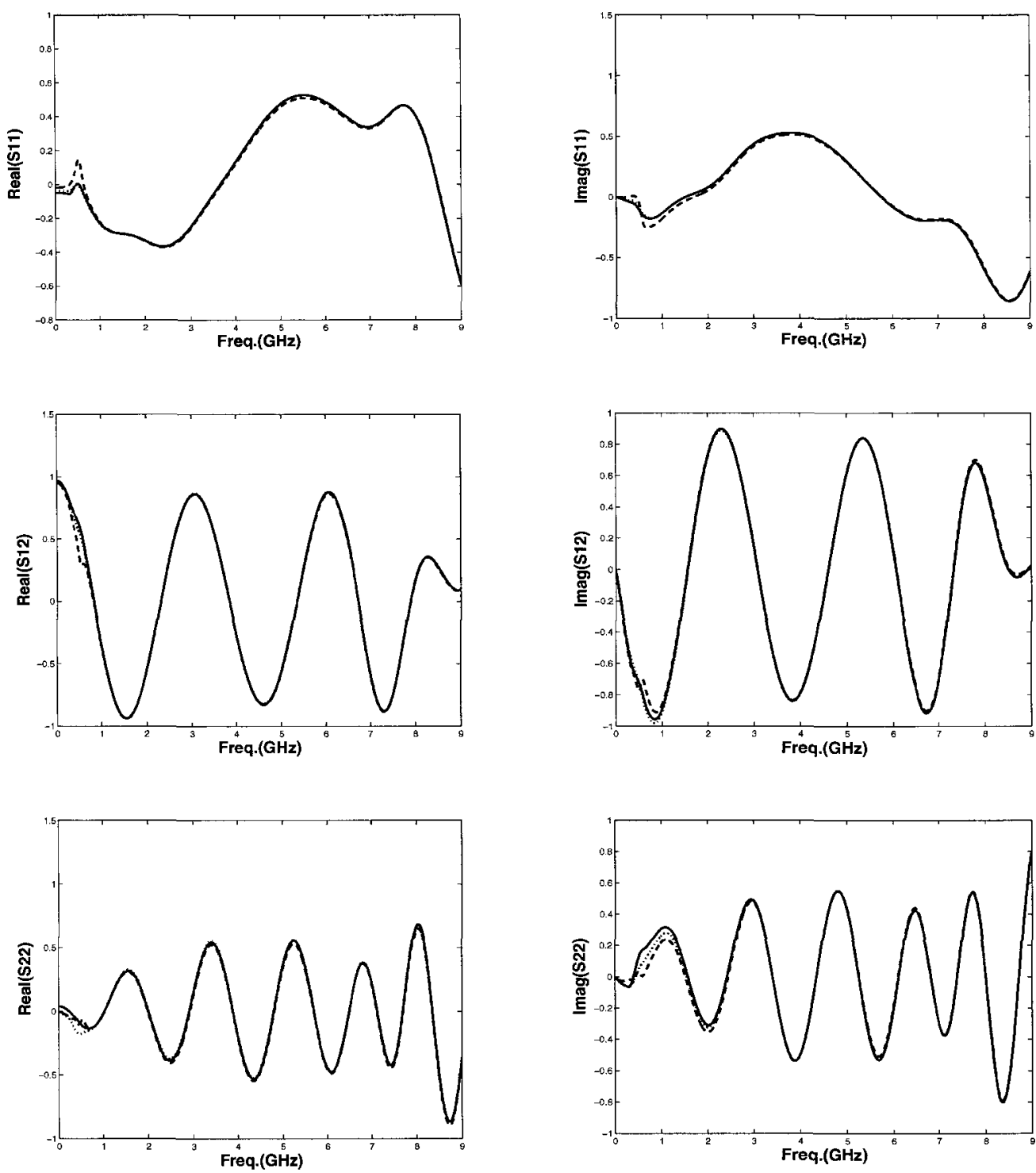

Fig. 4.11. S-parameter responses: Coaxial to microstrip coupler 


\subsubsection{Example 3: Two Coupled Lines}

For this example, the tabulated data (up to $5 \mathrm{GHz}$ ) of a coupled microstrip line, shown in Fig. 4.12, was considered. As seen by the plot of 2-norm of the scattering matrix versus frequency in Fig. 4.13 (solid line), the original data was not bounded-real (passive) at many frequency points. In this case the tabulated data was approximated by a common pole-set of 6 poles, which resulted in a statespace system of order 24 . The macromodel so obtained was found to be nonpassive and was compensated using the proposed passivity checking and enforcement algorithm (described in Section 4.2.2, Section 4.2.3 and Section 4.2.4). This process took 2 minutes for the example. Fig. 4.13 shows a comparison of the 2-norm of the scattering matrix versus frequency of the original data and macromodel before and after compensation. Some of the $S$-parameter responses of the macromodel before and after compensation are shown in Fig. 4.14. The relative norm of the perturbation of the residue matrix $\|\Delta C\| /\|C\|$ for this example was $1.1 \times 10^{-3}$. 


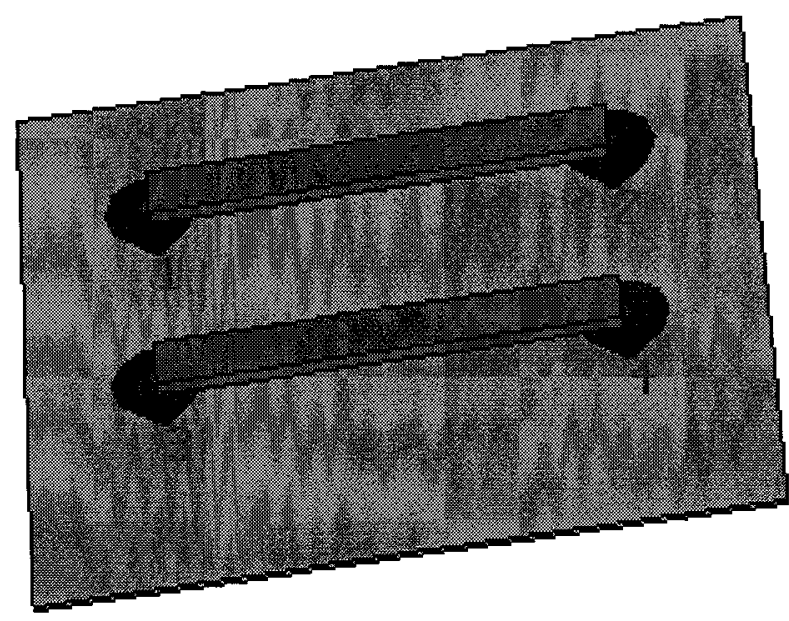

Fig. 4.12. Coupled microstrip line

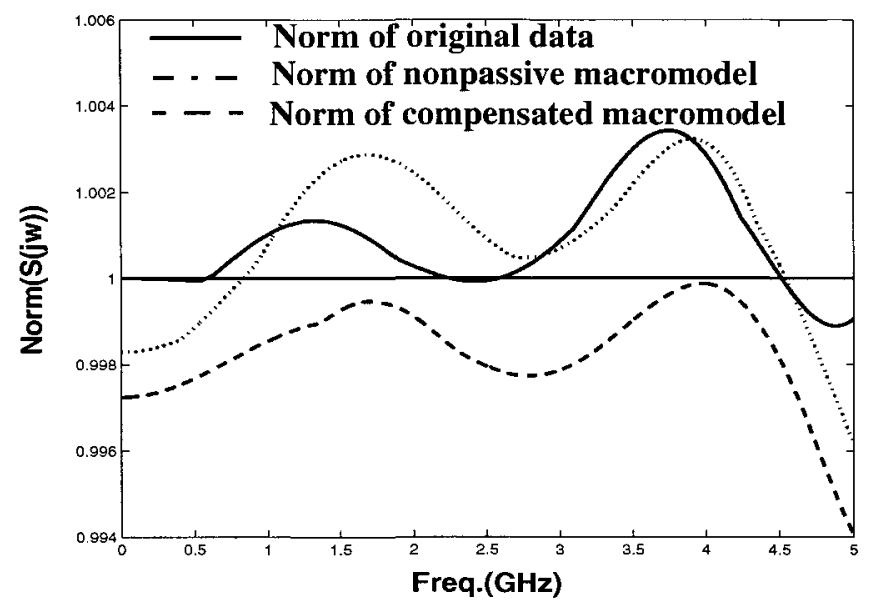

Fig. 4.13. Comparison of norm of $S(j \omega)$ : Coupled microstrip line 
Original data

..... Response before compensation

- - Response after compensation
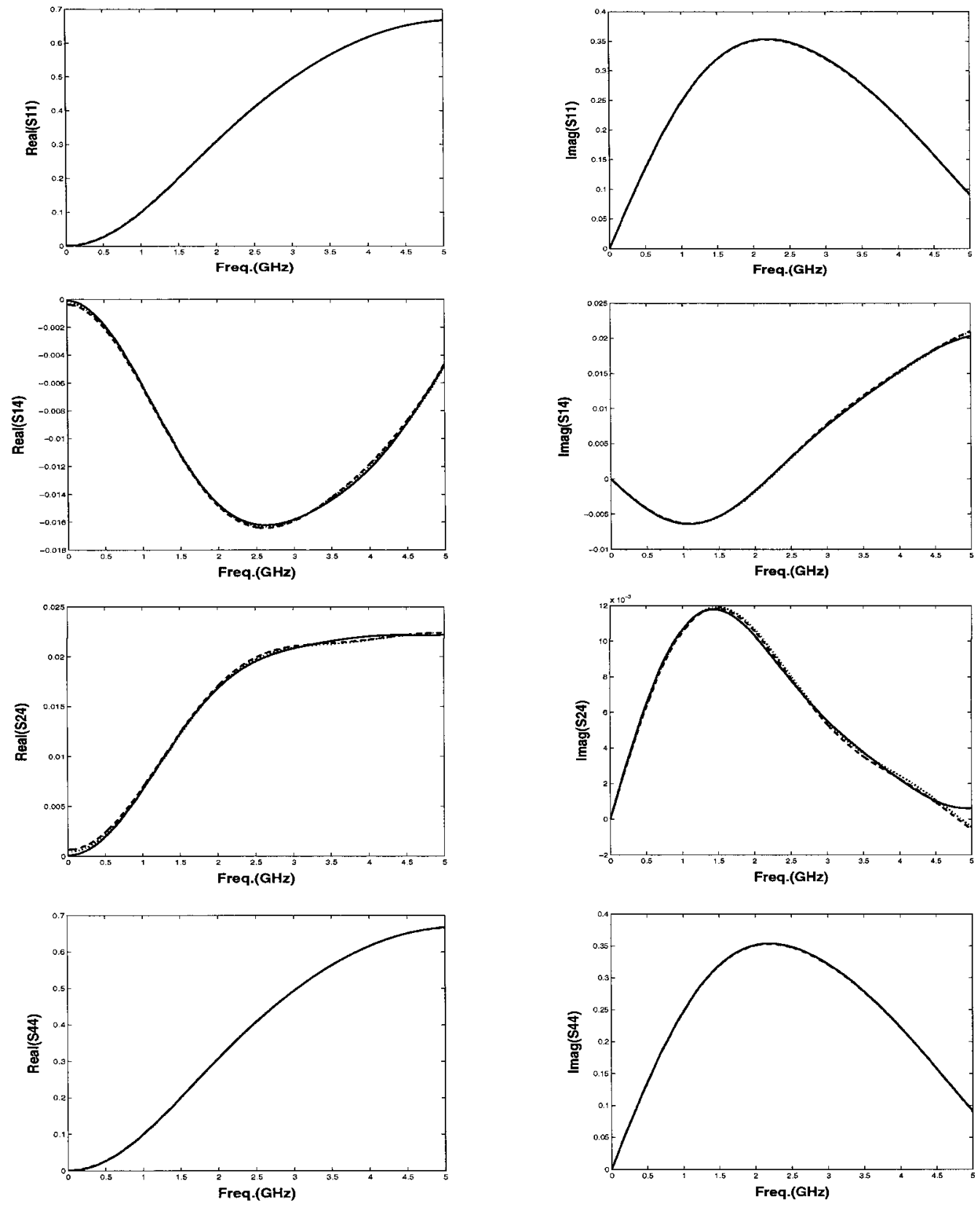

Fig. 4.14. S-parameter responses: Coupled microstrip line 


\subsubsection{Example 4: RJ-45 Connector}

In this example, the measured data of a 8-port RJ-45 connector, shown in Fig. 4.15, was considered. The data was approximated by a common pole-set of 10 poles, resulting in the state-space system of order 80 . The macromodel was found to be nonpassive using the proposed passivity check in Section 4.2.2. This is also demonstrated graphically in Fig. 4.16, where $\|\boldsymbol{S}(j \omega)\|_{2}$ exceeds value of one at several frequency locations, implying that the macromodel is violating the passivity criteria. The nonpassive macromodel was compensated for passivity violations using the proposed algorithm of Section 4.2.4. In each iteration of the compensation process, passivity check and quantification was done as outlined in Section 4.2.3, and the entire compensation process took 6.3 minutes. The response of the macromodel before and after compensation is shown in Fig. 4.17. The relative norm of the perturbation of the residue matrix $\|\Delta C\| /\|C\|$ for this example was $8 \times 10^{-3}$. 

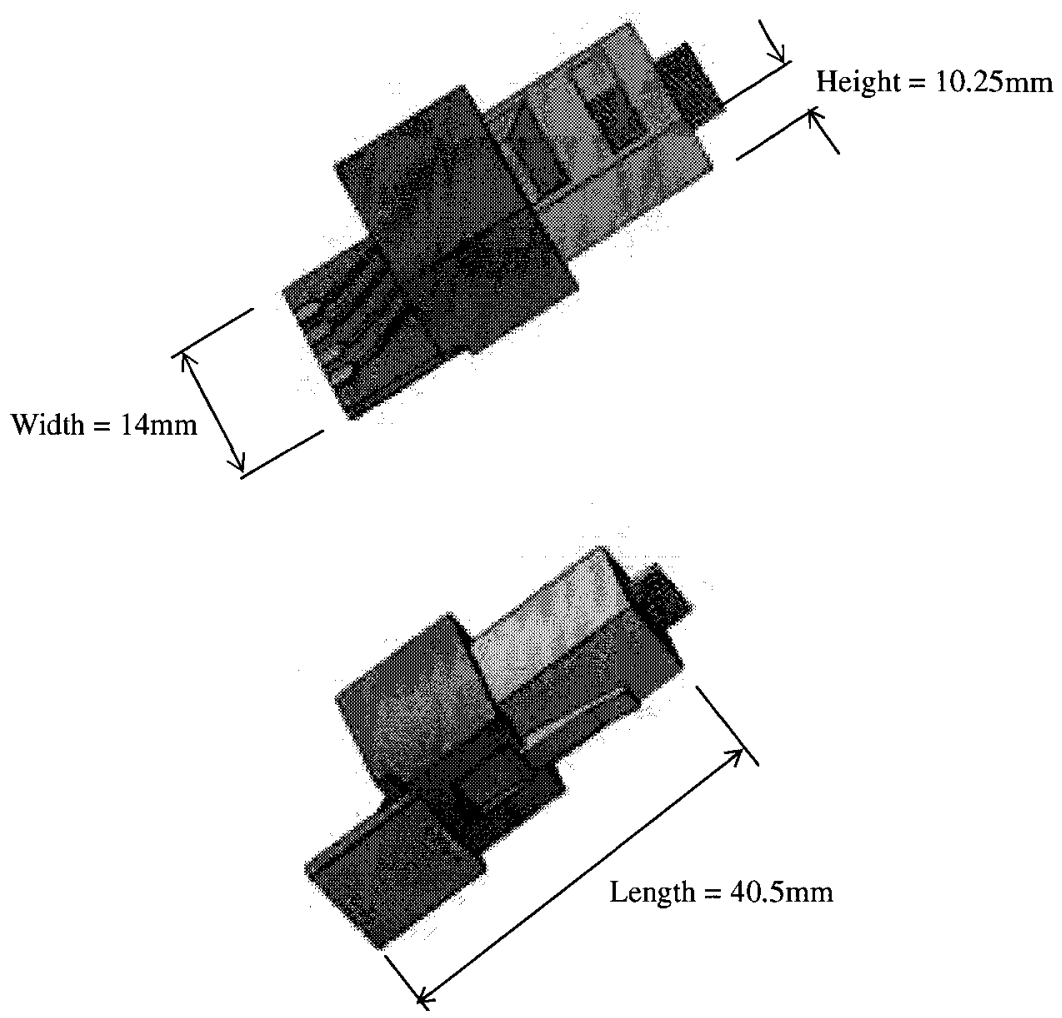

Fig. 4.15. RJ-45 connector

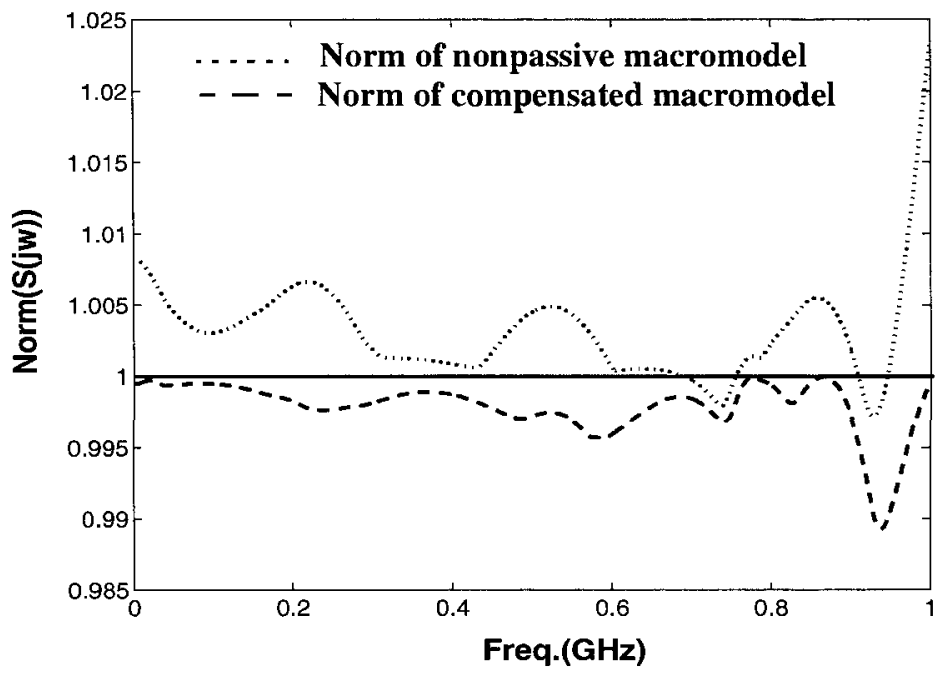

Fig. 4.16. Comparison of norm of $S(j \omega)$ : RJ-45 connector 


\section{Original data}

. . . . Response before compensation

- - Response after compensation
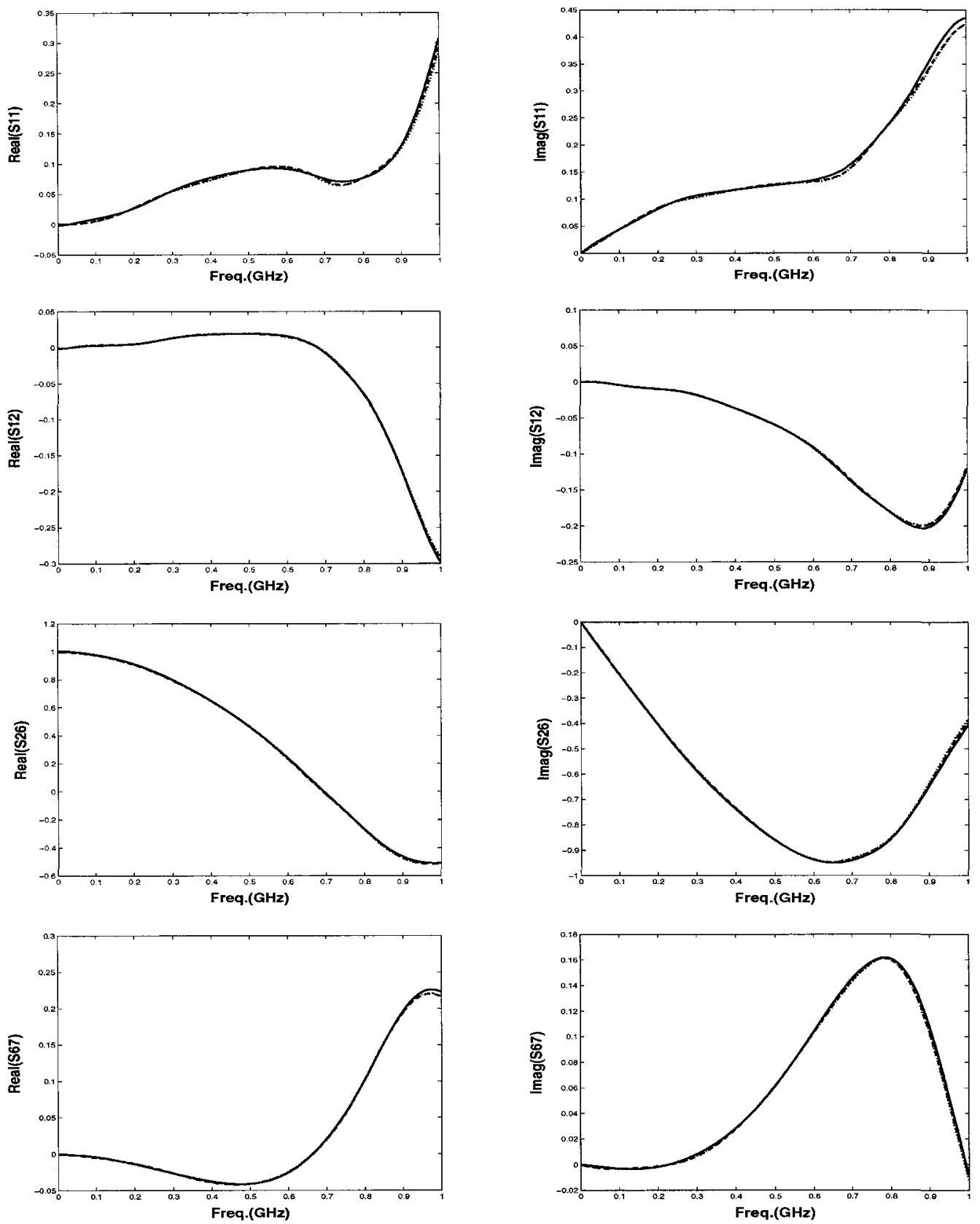

Fig. 4.17. S-parameter responses: $R J-45$ connector 


\subsubsection{Example 5: Multilayer Oval Via}

In this example, the measured data of a 6 -port multilayer oval via was considered. The dimensions of via used are as follows: Length $=500 \mathrm{mil}$, Height $=109 \mathrm{mil}$, Width $=300 \mathrm{mil}$. The tabulated $S$-parameter data was given up to $20 \mathrm{GHz}$. Accurate approximation of the data was achieved using a macromodel of order $32 \times 6=192$, employing the algorithm of Section 4.2.1. However, the macromodel was found to be nonpassive. The proposed algorithm for passivity check and enforcement described in Section 4.2.2 and Section 4.2.4 took 100 minutes. Fig. 4.18 shows the norm of the original data and the macromodel before and after compensation. The $S$-parameter responses of the nonpassive and passive macromodel are shown in Fig. 4.19 along with the original data. It is evident that the passive macromodel approximates the original data accurately. This is also reflected by the relative perturbation of the residue matrix $\|\Delta C\| /\|C\|$, whose value is equal to $9 \times 10^{-3}$.

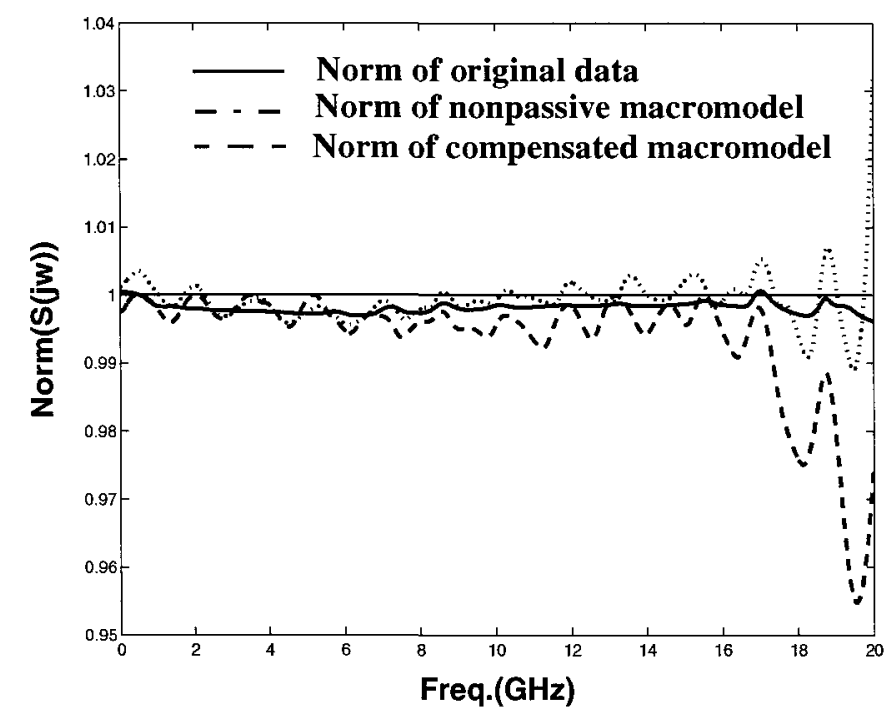

Fig. 4.18. Comparison of norm of $S(j \omega)$ : Multilayer oval via 
- Original data

. . . . Response before compensation

- - - Response after compensation
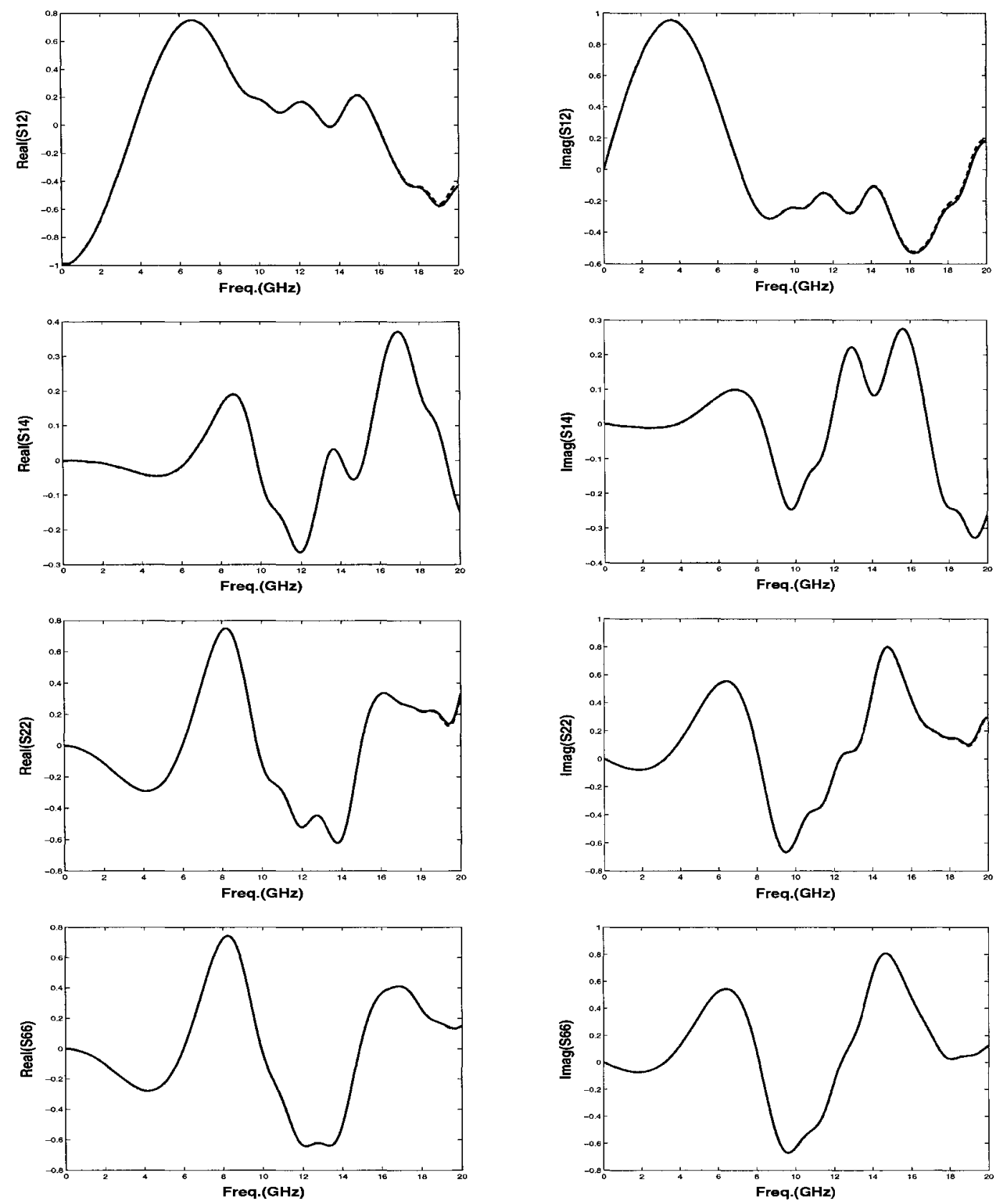

Fig. 4.19. S-parameter responses: Multilayer oval via 


\subsection{Conclusion}

In this chapter, an efficient technique for the passive macromodeling of tabulated networks was presented. It consists of a robust algebraic test for passivity and passivity enforcement algorithms for nonpassive macromodels. It is practical, robust and can be implemented easily in a simulator engine.

The major cost of the above algorithm is calculating the imaginary eigenvalues of the Hamiltonian matrix, whose size is two times that of the macromodel. Hence, it is ideally suited for small/medium scale systems with sizes in the order of few hundreds. However, when the size of the macromodel becomes large, the computational cost rises sharply because of the eigenvalue analysis of associated large Hamiltonian matrix. This is the typical case for large multiport networks with broadband responses. This issue is addressed in the next chapter and a fast technique for passivity verification and enforcement of large size macromodels is presented. 


\section{CHAPTER 5 \\ Passive Macromodeling of Tabulated Networks with Large-Order Macromodels}

The previous chapter presented the passivity checking and enforcement algorithm for small/medium size macromodels of tabulated networks. In this chapter, a computationally efficient algorithm is presented for the passive macromodeling of large order tabulated networks.

It is to be noted that, the broadband approximation of large multiport distributed interconnect networks can typically lead to macromodels with thousands of states. This can make the robust passivity checking based on Hamiltonian formulation and the subsequent passivity enforcement algorithm described in chapter 4 to be significantly slower. This is because, the presented algorithm needs to test if the macromodel is passive or not during each iteration of the passivity compensation process. This is done (as described in chapter 4) by finding the imaginary eigenvalues of the Hamiltonian matrix (which is of size twice that of the state matrix of the macromodel). In such a case, evaluating the imaginary eigenvalues of the Hamiltonian matrix may take considerable time.

For example, calculating the eigenvalues of a Hamiltonian matrix of size $2976 \times 2976$ required 16 minutes using eig function of MATLAB on a $2 \mathrm{GHz}$ computer with $1 \mathrm{~GB}$ of RAM. Using direct eigensolver, can therefore cause the passivity compensation process to run for several hours, reducing its practicality. 
Employing recent fast Hamiltonian eigen solvers such as [54], [55] (which employ selective hops combined with model-order reduction to evaluate the imaginary eigenvalues of the Hamiltonian matrix) the corresponding cost can be reduced considerably (for example, 19 seconds for the above case). However, since the passivity compensation process typically requires many iterations (can be typically in the range of $10 \mathrm{~s}$ or $100 \mathrm{~s}$ ), with each iteration requiring passivity verification, even the above reduced cost incurred in calculating the Hamiltonian imaginary eigenvalues/eigenvectors can still add up to a higher computational cost.

In order to address this difficulty associated with large-order macromodels, in this chapter, an efficient algorithm is presented for fast passivity verification, determination of any passivity violation regions and efficient passivity compensation. The proposed technique combines the merits of the recent fast Hamiltonian eigensolvers [54], [55] with the newly developed fast frequency sweep based on reciprocal system formulation [53]. This leads to minimized number of eigenvalue evaluations of Hamiltonian matrix during the iterative passivity compensation process of large-order macromodels, leading to computational speed-up.

Also, to further reduce the cost involved during the passivity enforcement step of Section 4.2.4, when applied on large size systems, a new method is presented in this chapter. It is based on reducing the cost involved during the solution of Lyapunov equation (4.54). The direct solvers for Lyapunov equation [83] require approximately $15 n^{3}$ flops. In the proposed method, the solution complexity of the Lyapunov equation is greatly reduced by exploiting the Jordan canonical form of the associated state-space system. 


\subsection{Problem Formulation and Preliminaries}

As described in Section 4.2, as a first step, the rational approximation of $\boldsymbol{S}$ parameter data of a $m$-port subnetwork is obtained as

$$
S_{i j}(s)=\sum_{i=1}^{n} \frac{k_{i}^{i, j}}{s-p_{i}}+d^{i, j} ; \quad i, j=1,2, \ldots m
$$

where the residues $\left(k_{i}\right)$ and poles $\left(p_{i}\right)$ can be real or complex conjugate pairs, $n$ is the number of poles and residues, $d$ is the real constant. In order that the model is bounded real (passive) at $\omega=\infty$, a constrained vector-fitting [52] is used, whereby it is guaranteed that the norm of the matrix of direct coupling constants $\boldsymbol{D}=\left[d^{i, j}\right] ; \quad(i, j \in 1 \ldots m)$ is less than one. The transfer function described by pole-residue model (5.1) is translated into a time-domain form described by ordinary differential equations (ODEs) as

$$
\begin{aligned}
& \dot{\boldsymbol{x}}(t)=\boldsymbol{A} \boldsymbol{x}(t)+\boldsymbol{B} \boldsymbol{a}(t) \\
& \boldsymbol{b}(t)=\boldsymbol{C} \boldsymbol{x}(t)+\boldsymbol{D} \boldsymbol{a}(t)
\end{aligned}
$$

where $\boldsymbol{x}(t) \in \mathfrak{R}^{q \times 1}$ (with $q=n m$ ) is the state vector, $\boldsymbol{a}(t) \in \mathfrak{R}^{m \times 1}$ is the input vector. $\boldsymbol{A} \in \mathfrak{R}^{q \times q}$ is the state-matrix, $\boldsymbol{B} \in \mathfrak{R}^{q \times m}$ is the matrix that relates the inputs to state-variables, $C \in \mathfrak{R}^{m \times q}$ is the matrix relating state variables to the outputs $(\boldsymbol{b}(t))$ and $\boldsymbol{D} \in \Re^{m \times m}$ is a matrix of direct coupling constants. The relationship between the input $\boldsymbol{a}(s)$ and output $\boldsymbol{b}(s)$ in frequency domain can be written as

$$
\boldsymbol{S}(s)=\boldsymbol{C}(s \boldsymbol{I}-\boldsymbol{A})^{-1} \boldsymbol{B}+\boldsymbol{D}
$$


Given a matrix-transfer function described by (5.1), several forms of time-domain realizations (5.2) can be obtained. In the proposed algorithm the time-domain realization is accomplished using the Jordan-canonical [79], [80] form. For the purpose of illustration, the form of the state-space matrices using Jordancanonical realization is given below, for the case of a two port network with one real and one complex pole-pair.

Let the two port network contain one real pole-residue pair $\left(p_{1}, k_{1}\right)$, one complexconjugate set of pole-residue pair $\left(p_{2,3}=w \pm i z ; \quad k_{2,3}=r \pm i v\right)$ and the direct coupling constant $d$. The Jordan-canonical realization in this case can be written as

$$
\begin{aligned}
& {\left[\begin{array}{l}
\dot{x}_{1} \\
\dot{x}_{2} \\
\dot{x}_{3} \\
\dot{x}_{4}
\end{array}\right]=\left[\begin{array}{cccccc}
w & 0 & z & 0 & 0 & 0 \\
0 & w & 0 & z & 0 & 0 \\
-z & 0 & w & 0 & 0 & 0 \\
0 & -z & 0 & w & 0 & 0 \\
0 & 0 & 0 & 0 & p_{1} & 0 \\
0 & 0 & 0 & 0 & 0 & p_{1}
\end{array}\right]\left[\begin{array}{l}
x_{1} \\
x_{2} \\
x_{3} \\
x_{4}
\end{array}\right]+\left[\begin{array}{ll}
2 & 0 \\
0 & 2 \\
0 & 0 \\
0 & 0 \\
1 & 0 \\
0 & 1
\end{array}\right]\left[\begin{array}{l}
a_{1} \\
a_{2}
\end{array}\right]} \\
& \boldsymbol{y}=\left[\begin{array}{l}
b_{1} \\
b_{2}
\end{array}\right]=\left[\begin{array}{cccccc}
r^{1,1} & r^{1,2} & v^{1,1} & v^{1,2} & k_{1}^{1,1} & k_{1}^{1,2} \\
r^{2,1} & r^{2,2} & v^{2,1} & v^{2,2} & k_{1}^{2,1} & k_{1}^{2,2}
\end{array}\right]\left[\begin{array}{l}
x_{1} \\
x_{2} \\
x_{3} \\
x_{4}
\end{array}\right]+\left[\begin{array}{ll}
d^{1,1} & d^{1,2} \\
d^{2,1} & d^{2,2}
\end{array}\right]
\end{aligned}
$$




\subsection{Proposed Algorithm for Passivity Verification/ Enforcement of Large Order Macromodels}

For handling large-order macromodels, a fast passivity verification and enforcement algorithm is presented in this section. The proposed passivity verification does not use the expensive algebraic tests based on the eigen analysis of Hamiltonian matrix in every iteration. Rather, it uses an efficient closed-form adaptive frequency sweep of the macromodel response to obtain the information about the regions of passivity violation (for $\omega=0 \ldots \infty$ ). Once the regions of violation are compensated, a call to the Hamitonian based passivity check is made. As a result, the number of calls to the eigen analysis of Hamiltonian matrix are minimized. Details are given below.

\subsubsection{Quantification of Passivity Violation for Large Scale Systems}

In this section, a practical and computationally efficient technique for the passivity checking and quantification of passivity violation is presented [53]. It is based on the intelligent frequency sweep of $\|S(j \omega)\|_{2}$ versus $\omega$. Unlike the Hamiltonian matrix, which can be very large and dense (in the order of thousands), the scattering matrix $\boldsymbol{S}(j \omega)$ is of small size, which is purely decided by the number of ports. This makes the frequency sweep of $\|S(j \omega)\|_{2}$ an attractive option for the passivity verification of large macromodels. However, two major issues arise when considering such a frequency sweep:

1. Determination of the maximum frequency for the sweep. Determining this frequency may not be trivial, since theoretically the response need to be swept up to infinity. 
2. How to identify the magnitude of the maximum violation in a non-passive region.

To overcome the above difficulties, a new methodology based on a reciprocal system is presented below.

\subsection{1a Frequency-Sweep of the Region Between $f_{\max }$ and $\infty$ via Reciprocal Systems}

Let $f_{\max }$ represent the frequency up to which the tabulated data is given. Fast frequency sweep form 0 to $f_{\max }$ can be performed using the pole-residue formulation (5.1) using direct substitution. However, it is not trivial to perform the frequency sweep between $f_{\max }$ and $\infty$ to detect the passivity violation. For the purpose of illustration, let $f_{v}$ be a sample unknown frequency in the band between $f_{\max }$ and $\infty$, at which the bounded-real condition is violated (shown in Fig. 5.1(a)). In the proposed method, the problem of detecting $f_{v}$ is accomplished by constructing a reciprocal system. Here $\infty$ of the original frequency-domain is mapped to the zero of the reciprocal-domain and vice-versa. This implies that, $f_{\max }$ of the original domain is mapped to $1 / f_{\max }$ of reciprocal domain. This simplifies the task of sweeping from $f_{\max }$ to $\infty$ in the original domain to sweeping between $1 / f_{\max }$ and zero of the reciprocal domain (demonstrated in Fig. 5.1(b)). 


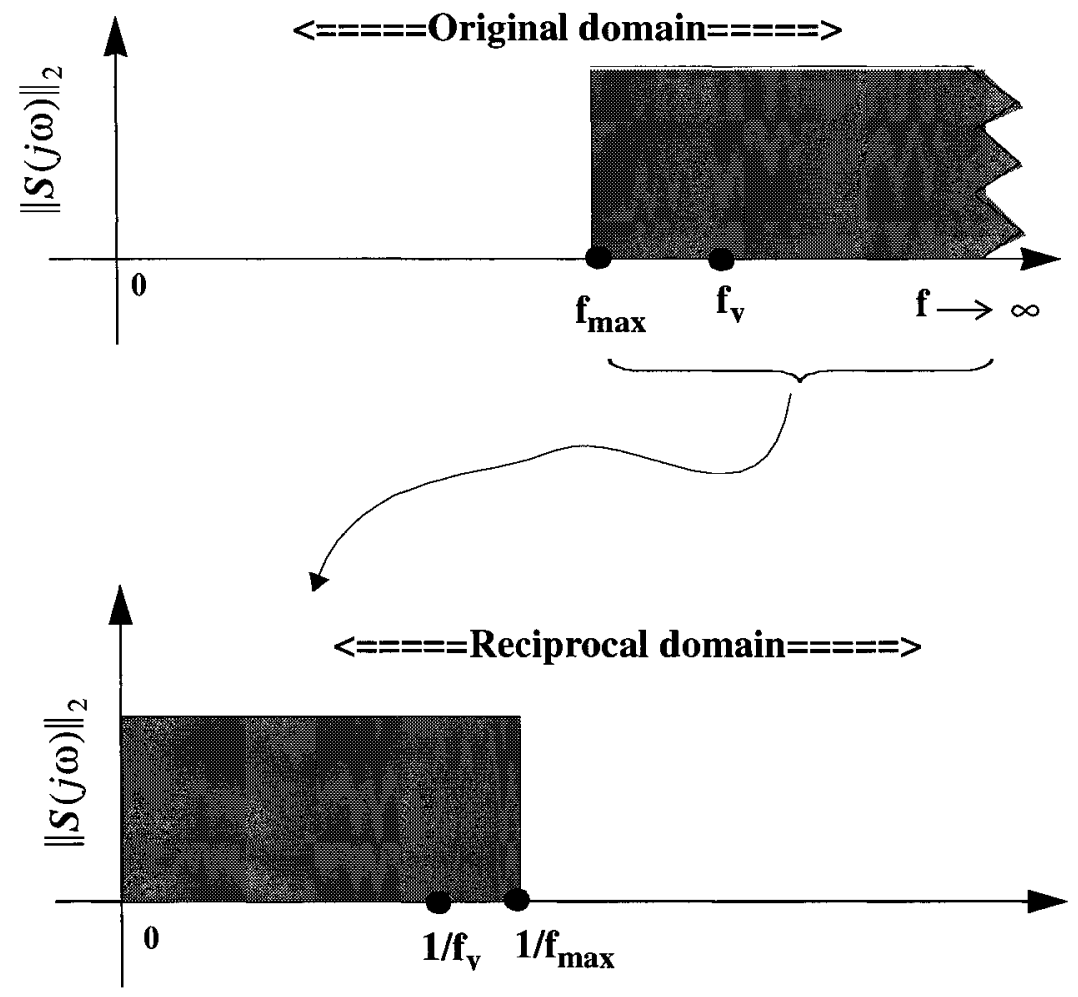

(a)

(b)

Fig. 5.1. Mapping from original domain to reciprocal domain

The reciprocal system $\left(S_{r}(s)\right)$ can be constructed by substituting $s$ with $1 / s$ in the original system of (5.3) as

$$
\boldsymbol{S}_{r}(s)=\boldsymbol{C}\left(s^{-1} \boldsymbol{I}-\boldsymbol{A}\right)^{-1} \boldsymbol{B}+\boldsymbol{D}
$$

Next, $\left(s^{-1} \boldsymbol{I}-\boldsymbol{A}\right)^{-1}$ can be written as [84]

$$
\left(s^{-1} \boldsymbol{I}-\boldsymbol{A}\right)^{-1}=-\boldsymbol{A}^{-1}-\boldsymbol{A}^{-1}\left(s \boldsymbol{I}-\boldsymbol{A}^{-1}\right)^{-1} \boldsymbol{A}^{-1}
$$

Substituting (5.7) in (5.6), the reciprocal system can be written as

$$
S_{r}(s)=-\boldsymbol{C A}^{-1} \boldsymbol{B}-\boldsymbol{C A}^{-1}\left(s \boldsymbol{I}-\boldsymbol{A}^{-1}\right)^{-1} \boldsymbol{A}^{-1} \boldsymbol{B}+\boldsymbol{D}
$$


or

$$
\boldsymbol{S}_{r}(s)=\boldsymbol{C}_{r}\left(s \boldsymbol{I}-\boldsymbol{A}_{r}\right)^{-1} \boldsymbol{B}_{r}+\boldsymbol{D}_{r}
$$

From (5.8) and (5.9) it is evident that the state-space matrices of the reciprocal system are

$$
\boldsymbol{A}_{r}=\boldsymbol{A}^{-1} ; \quad \boldsymbol{B}_{r}=-\boldsymbol{A}^{-1} \boldsymbol{B} ; \quad \boldsymbol{C}_{r}=\boldsymbol{C A ^ { - 1 }} ; \quad \boldsymbol{D}_{r}=\boldsymbol{D}-\boldsymbol{C A ^ { - 1 } \boldsymbol { B }}
$$

\subsection{1b Computational Considerations of Constructing the Reciprocal System}

In order to reduce the computational cost involved in constructing the reciprocal system, properties of the macromodel derived from pole-residue models are exploited (which is typically the case in macromodeling of high-frequency modules, where the first step is to obtain a pole-residue model using algorithms such as, vector-fitting). As described in Section 5.1, the state-space system $\boldsymbol{A}, \boldsymbol{B}, \boldsymbol{C}, \boldsymbol{D}$ is typically in the canonical form of (5.4), (5.5). For this form, the inverse of matrix $\boldsymbol{A}$ can be obtained analytically. For example, consider the state matrix $\boldsymbol{A}$ in (5.4). Its inverse can be written in a closed form as 


$$
A_{r}=A^{-1}=\left[\begin{array}{cccccc}
\frac{w}{w^{2}+z^{2}} & 0 & -\frac{z}{w^{2}+z^{2}} & 0 & 0 & 0 \\
0 & \frac{w}{w^{2}+z^{2}} & 0 & -\frac{z}{w^{2}+z^{2}} & 0 & 0 \\
\frac{z}{w^{2}+z^{2}} & 0 & \frac{w}{w^{2}+z^{2}} & 0 & 0 & 0 \\
0 & \frac{z}{w^{2}+z^{2}} & 0 & \frac{w}{w^{2}+z^{2}} & 0 & 0 \\
0 & 0 & 0 & 0 & \frac{1}{p_{1}} & 0 \\
0 & 0 & 0 & 0 & 0 & \frac{1}{p_{1}}
\end{array}\right]
$$

Also, it should be noted that $\boldsymbol{A}_{r}$ retains the same sparse pattern as that of the original state matrix. Hence, all that is required to create the rest of the reciprocal system $\left(\boldsymbol{B}_{r}, \boldsymbol{C}_{r}, \boldsymbol{D}_{r}\right)$ is a set of sparse matrix-vector multiplications as defined by $(5.10)$.

\subsection{1c Computational Considerations of Frequency Sweep}

Frequency sweep needs to be performed both on the original system (from 0 to $f_{\text {max }}$ ) and on the reciprocal system (from 0 to $1 / f_{\text {max }}$ ). For the original system $(\boldsymbol{A}, \boldsymbol{B}, \boldsymbol{C}, \boldsymbol{D})$, it can be seen that the frequency sweep of the norm-spectrum $\left(\|S(j \omega)\|_{2}\right)$ can be obtained either by evaluating (5.1) or (5.3). However, for large systems, evaluating (5.3) at many frequency points may take a long time as compared to evaluating (5.1). It is to be noted that, (5.1) is readily available as the first output of most approximation algorithms, such as vector-fit [26]. For example, sweeping the response at 500 frequency points, of a 12-port system with macromodel of size $1488 \times 1488$ (details in Section 5.5.2) required 8 minutes using 
(5.3). On the other hand, evaluating (5.1) for the same system and at the same number of points required only 0.9 seconds. Hence, in the work presented in this dissertation, it is proposed to perform the frequency sweep using the closed-form expression (5.1).

But, when it comes to sweeping the reciprocal system (5.9), we do not yet have the desired computational efficient form of (5.1). In order to overcome this difficulty, a closed-form relationship is developed using the properties of the reciprocal system, to represent it in the form of (5.1). To achieve this, we need the information about the eigenvalues and eigenvectors of the reciprocal state matrix $\boldsymbol{A}_{r}$. Since $\boldsymbol{A}_{r}$ is available in canonical form, its eigen (or Schur) decomposition can be obtained in a closed-form [80]. For the purpose of illustration, consider the reciprocal state matrix $\left(\boldsymbol{A}_{r}\right)$ of (5.11) (which is of canonical form). Its Schur decomposition can be written in a closed-form as

$$
\boldsymbol{A}_{r}=\boldsymbol{U}_{r} \boldsymbol{T}_{r} \boldsymbol{U}_{r}^{H}
$$

where

$$
\boldsymbol{U}_{r}=\left[\begin{array}{cccccc}
\frac{1}{\sqrt{2}} & 0 & \frac{1}{\sqrt{2}} & 0 & 0 & 0 \\
0 & \frac{1}{\sqrt{2}} & 0 & \frac{1}{\sqrt{2}} & 0 & 0 \\
-\frac{j}{\sqrt{2}} & 0 & \frac{j}{\sqrt{2}} & 0 & 0 & 0 \\
0 & -\frac{j}{\sqrt{2}} & 0 & \frac{j}{\sqrt{2}} & 0 & 0 \\
0 & 0 & 0 & 0 & 1 & 0 \\
0 & 0 & 0 & 0 & 0 & 1
\end{array}\right] ; \quad \boldsymbol{U}_{r}{ }^{H} \boldsymbol{U}_{r}=\boldsymbol{I}
$$




$$
\boldsymbol{T}_{r}=\left[\begin{array}{cccccc}
\frac{w+j z}{w^{2}+z^{2}} & 0 & 0 & 0 & 0 & 0 \\
0 & \frac{w+j z}{w^{2}+z^{2}} & 0 & 0 & 0 & 0 \\
0 & 0 & \frac{w-j z}{w^{2}+z^{2}} & 0 & 0 & 0 \\
0 & 0 & 0 & \frac{w-j z}{w^{2}+z^{2}} & 0 & 0 \\
0 & 0 & 0 & 0 & \frac{1}{p_{1}} & 0 \\
0 & 0 & 0 & 0 & 0 & \frac{1}{p_{1}}
\end{array}\right]
$$

The above Schur decomposition likewise is analytically available for any size of matrix $\boldsymbol{A}_{r}$, that is in the canonical form. Next, let $\boldsymbol{T}_{r}=\operatorname{diag}\left[\begin{array}{llll}\lambda_{1} & \lambda_{2} & \ldots & \lambda_{q}\end{array}\right]$ where the diagonal elements represent the eigenvalues of matrix $\boldsymbol{A}_{r}$ (as illustrated in (5.14)) and the matrix $\boldsymbol{U}_{r}$ contains the corresponding eigenvectors (given by (5.13)). Using (5.12), (5.13) and (5.14) the reciprocal system represented by (5.9) can be re-written as

$$
\begin{aligned}
\boldsymbol{S}_{r}(s)=\boldsymbol{C}_{r}\left(s \boldsymbol{I}-\boldsymbol{A}_{r}\right)^{-1} \boldsymbol{B}_{r}+\boldsymbol{D}_{r} & =\boldsymbol{C}_{r}\left(s \boldsymbol{I}-\boldsymbol{U}_{r} \boldsymbol{T}_{r} \boldsymbol{U}_{r}^{-1}\right)^{-1} \boldsymbol{B}_{r}+\boldsymbol{D}_{r} \\
& =\boldsymbol{C}_{r} \boldsymbol{U}_{r}\left(s \boldsymbol{I}-\boldsymbol{T}_{r}\right)^{-1} \boldsymbol{U}_{r}^{-1} \boldsymbol{B}_{r}+\boldsymbol{D}_{r} \\
& =\boldsymbol{C}_{r} \boldsymbol{U}_{r}\left[\begin{array}{cc}
\frac{1}{s-\lambda_{1}} & \\
\cdots & \\
& \frac{1}{s-\lambda_{q}}
\end{array}\right] \boldsymbol{U}_{r}^{-1} \boldsymbol{B}_{r}+\boldsymbol{D}_{r}
\end{aligned}
$$

which can be simplified as 


$$
\boldsymbol{S}_{r}(s)=\sum_{i=1}^{q} \frac{\boldsymbol{k}_{i}^{r}}{s-\lambda_{i}}+\boldsymbol{D}_{r}
$$

where the residue matrix $\boldsymbol{k}_{i}^{r}$ is a function of $\boldsymbol{B}_{r}, \boldsymbol{C}_{r}$ and eigenvectors of matrix $\boldsymbol{A}_{r}$. Using the closed-form representation of the reciprocal system given by (5.16), a fast frequency sweep can be accomplished.

\subsection{1d Adaptive Approach for Determining the Magnitude of the Maximum Violation}

Once the regions of passivity violations are identified, the magnitude of the maximum violations in each region can be quickly accomplished by performing a fine sweep in the portion of peaks of the regions of passivity violation. This is pictorially illustrated in Fig. 5.2. Since the sweep is done using closed-form relations, it is computationally efficient.

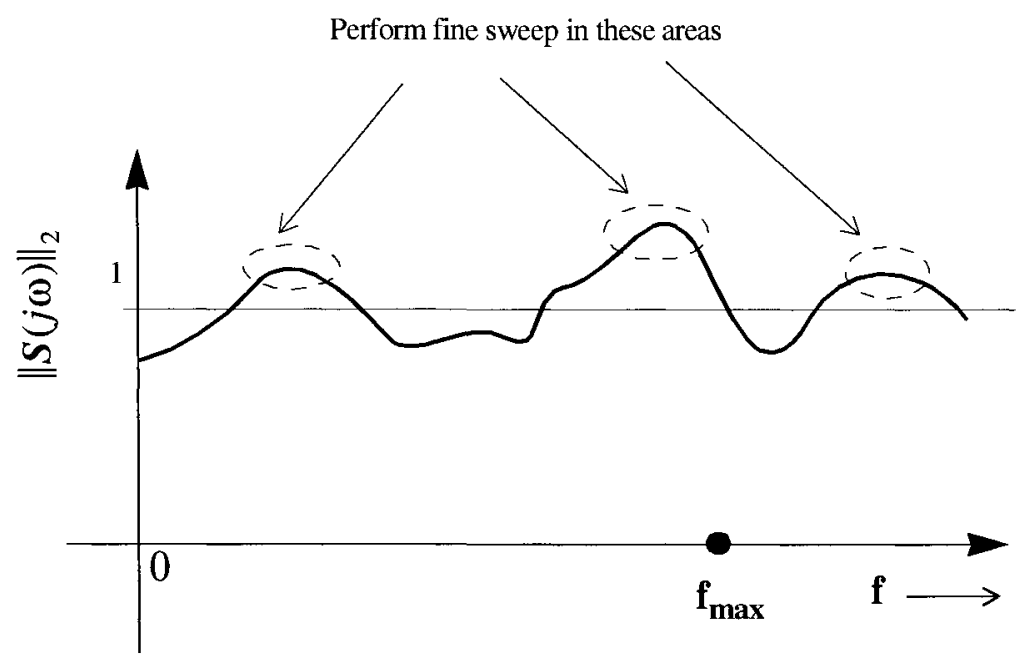

Fig. 5.2. Determination of locations of maximum passivity violation 


\subsection{Proposed Passivity Enforcement}

With the information about passivity violation locations from Section 5.2.1, the macromodel is compensated for passivity violation. The passivity compensation algorithms described in this section perturb the residue matrix $\boldsymbol{C}$ such that the resulting macromodel is passive and also satisfies the accuracy constraints of the approximation. The steps are similar to the PCFOP (Section 4.2.4a) and PCLMI (Section 4.2.4b) approaches outlined in chapter 4.

In effect, the following problem is solved iteratively at the frequency points of violation, to satisfy the passivity criteria:

$$
\begin{gathered}
\min \left(\boldsymbol{X}^{T} \boldsymbol{H} \boldsymbol{X}\right) ; \quad \text { such that }, \\
\Delta \boldsymbol{C} \\
\boldsymbol{W} \boldsymbol{X}=\Delta \lambda
\end{gathered}
$$

where,

$$
\boldsymbol{H}=\left[\begin{array}{cccc}
\boldsymbol{P} & 0 & \ldots & 0 \\
0 & \boldsymbol{P} & \ldots & 0 \\
& & \ldots & \\
0 & 0 & \ldots & \boldsymbol{P}
\end{array}\right]_{(q m) \times(q m)}
$$

and $\boldsymbol{X}$ consists of the elements of $\boldsymbol{\Delta C}$ arranged in a column. During an iteration, $W$ consists of the information of all the frequency points where the passivity correction is desired. As such $\boldsymbol{W} \in \mathfrak{R}^{n_{f} \times q m}$, with $n_{f}$ representing the number of frequency points where the passivity correction is being done and $q=n m$ is the order of the system, with $n$ the number of poles in the common pole-set. It is evident that the objective function in (5.17) is quadratic in nature and therefore 
can be solved using efficient routines directed specifically towards minimizing the quadratic function.

It is to be noted that, during each iteration, passivity correction is accomplished by perturbing only the residues. Hence, while performing the frequency sweep of the original system for next iteration, closed-form of (5.1) is used simply by replacing the old residues by the updated residues. For the reciprocal system, the updated residues for (5.16) are obtained using sparse vector-matrix multiplications defined by (5.10) and (5.15).

As discussed in Section 4.2.4, an important aspect for minimizing the error introduced in the response of the macromodel during the compensation process, is the inclusion of a cost function as a constraint in these compensation techniques. This cost function involves the computation of the controllability Grammian of the system $\boldsymbol{P}$, by solving Lyapunov equation ((4.54))

$$
\boldsymbol{A P}+\boldsymbol{P} \boldsymbol{A}^{H}+\boldsymbol{B} \boldsymbol{B}^{H}=\mathbf{0}
$$

For large size macromodels, computation of this Grammian by standard Lyapunov solver algorithms may be computationally expensive. For example, solving (5.19) for a system with $\boldsymbol{A}$-matrix of size $1488 \times 1488$ required 14.3 minutes using lyap function of MATLAB on a $2 \mathrm{GHz}$ computer with 1GB of RAM (details in Section 5.5.2). Hence, in order to overcome this difficulty, a fast solution method is presented below. It takes advantage of the canonical forms of the statespace matrices $\boldsymbol{A}$ and $\boldsymbol{B}$, of the proposed algorithm, resulting in substantial cost reduction in solving Lyapunov equations. 


\subsection{1a Fast Solution of Lyapunov Equation}

In the proposed method, the solution complexity of the controllability Grammian $\boldsymbol{P}$ is greatly reduced by exploiting the Jordan canonical form of the associated state-space system $\boldsymbol{A}, \boldsymbol{B}, \boldsymbol{C}, \boldsymbol{D}$. The major cost in solving the Lyapunov equation is due to the Schur factorization of the matrix $\boldsymbol{A}$. This can be addressed for the case of the canonical form of matrix $\boldsymbol{A}$, since the Schur factors are analytically available (similar to the one given by (5.13), (5.14)). Exploiting this fact, the only cost in the solution of Lyapunov equation that remains, is solving a set of sparse linear uncoupled equations, as outlined below. Let the Schur decomposition of the matrix $\boldsymbol{A}$ be represented by:

$$
\boldsymbol{A}=\boldsymbol{U} \boldsymbol{T} \boldsymbol{U}^{H}
$$

For the purpose of illustration, consider the state matrix $\boldsymbol{A}$ in (5.4). Its Schur factorization (5.20) is analytically available, where

$$
\boldsymbol{U}=\left[\begin{array}{cccccc}
\frac{1}{\sqrt{2}} & 0 & \frac{1}{\sqrt{2}} & 0 & 0 & 0 \\
0 & \frac{1}{\sqrt{2}} & 0 & \frac{1}{\sqrt{2}} & 0 & 0 \\
\frac{j}{\sqrt{2}} & 0 & -\frac{j}{\sqrt{2}} & 0 & 0 & 0 \\
0 & \frac{j}{\sqrt{2}} & 0 & -\frac{j}{\sqrt{2}} & 0 & 0 \\
0 & 0 & 0 & 0 & 1 & 0 \\
0 & 0 & 0 & 0 & 0 & 1
\end{array}\right] ; \quad \boldsymbol{U}^{H} \boldsymbol{U}=\boldsymbol{I}
$$


and

$$
\boldsymbol{T}=\left[\begin{array}{cccccc}
w+j z & 0 & 0 & 0 & 0 & 0 \\
0 & w+j z & 0 & 0 & 0 & 0 \\
0 & 0 & w-j z & 0 & 0 & 0 \\
0 & 0 & 0 & w-j z & 0 & 0 \\
0 & 0 & 0 & 0 & p_{1} & 0 \\
0 & 0 & 0 & 0 & 0 & p_{1}
\end{array}\right]
$$

Substituting (5.20) in (5.19) and subsequently, pre-multiplying by $\boldsymbol{U}^{H}$ and post multiplying by $\boldsymbol{U}$ results in the following equation

$$
\boldsymbol{U}^{H} \boldsymbol{U} \boldsymbol{T} \boldsymbol{U}^{H} \boldsymbol{P} \boldsymbol{U}+\boldsymbol{U}^{H} \boldsymbol{P} \boldsymbol{U} \boldsymbol{T}^{H} \boldsymbol{U}^{H} \boldsymbol{U}=-\boldsymbol{U}^{H} \boldsymbol{B} \boldsymbol{B}^{H} \boldsymbol{U}
$$

Next, substituting $\hat{\boldsymbol{P}}=\boldsymbol{U}^{H} \boldsymbol{P} \boldsymbol{U}, \hat{\boldsymbol{B}}=\boldsymbol{U}^{H} \boldsymbol{B}$ and using the fact that $\boldsymbol{U}^{H} \boldsymbol{U}=\boldsymbol{I},(5.23)$ can be written as

$$
\boldsymbol{T} \hat{\boldsymbol{P}}+\hat{\boldsymbol{P}} \boldsymbol{T}^{H}=-\hat{\boldsymbol{B}} \hat{\boldsymbol{B}}^{H}
$$

Because of the form of matrices $\boldsymbol{T}, \boldsymbol{B}$ and $\boldsymbol{U}$ of the proposed state-space matrices, (5.24) results in uncoupled algebraic relations to solve for the matrix $\hat{\boldsymbol{P}}$. The solution $\boldsymbol{P}$ of the original Lyapunov equation (5.19) can be obtained as $\boldsymbol{P}=\boldsymbol{U} \hat{\boldsymbol{P}} \boldsymbol{U}^{H}$.

\subsection{1b Discussion of Computational Complexity}

Let $n_{i}$ be the number of iterations required for passivity compensation, while $n_{H}$ is the number of times the Hamiltonian based check is done in the proposed 
algorithm. In the proposed method $n_{H} \ll n_{i}$ (with a good implementation of the proposed algorithm, $n_{H}$ can be equal to 1$)$.

Each passivity compensation iteration of the proposed algorithm and the one presented in [43] and [54], involves two major parts. The first one is the passivity checking part, while the second one is quantifying the passivity violations and solving the compensation problem (5.17). The cost of the first part is greatly reduced in the proposed algorithm by minimizing the use of Hamiltonian eigenvalue evaluations. A summary of the computation cost comparison of the proposed passivity checking algorithm as compared to the techniques in [43] and [55] is presented below.

Table. 5.1. Comparison of computational complexity of passivity verification

\begin{tabular}{|c|c|c|}
\hline Full Hamiltonian based [43] & $\begin{array}{c}\text { Fast Hamiltonian eigenvalue } \\
\text { solver [54], [55] }\end{array}$ & $\begin{array}{c}\text { Proposed method } \\
{[47],[53]}\end{array}$ \\
\hline \hline$n_{i} \times O\left(n^{3}\right)$ & $n_{i} \times O(n)$ & $n_{H} \times O(n)$ \\
\hline
\end{tabular}

It is apparent that the proposed algorithm requires $\left(n_{i}-n_{H}\right) \times O(n)$ fewer operations as compared to the fastest methods available. The computation cost of the second part is $O(n)$ for the proposed algorithm as well as for [43], [54], [55].

\subsection{Guidelines for Passivity Verification During the Intermediate Steps of the Compensation Process for Large Scale Systems}

For large scale systems, use of the following guidelines can avoid unnecessary sweep of the entire spectrum during successive iterations, thereby resulting in additional computational savings. For the purpose of discussion, consider the 
norm-spectrum with multiple passivity violation regions as shown in shown Fig. 5.3.

1. To begin with, the region with highest violation is considered for passivity compensation (this corresponds to region-1 in Fig. 5.3). Since the immediate objective after each iteration is to ensure that the violation in the region under consideration is compensated for, it would be sufficient to do a fine sweep in the vicinity of region-1 (i.e. sweep between $\omega_{1}$ and $\omega_{2}$ ) to determine the extent of the magnitude of the new compensation required, thereby avoiding the sweep of the rest of the spectrum. This also implies that, there is no need to build/ sweep the updated reciprocal system of (5.16), during the intermediate steps of the compensation process.

2. After every identified passivity violation region (such as region-1 and region2 in Fig. 5.3) is compensated using successive iterations, a fine-sweep of the entire frequency spectrum is performed using both the updated original system of (5.1) and the updated reciprocal system of (5.16) to check if any new passivity violations were introduced during the passivity compensation process. If any new passivity violations were detected, then the step-1 is repeated. This process converges quickly as demonstrated in Section 5.5 . 


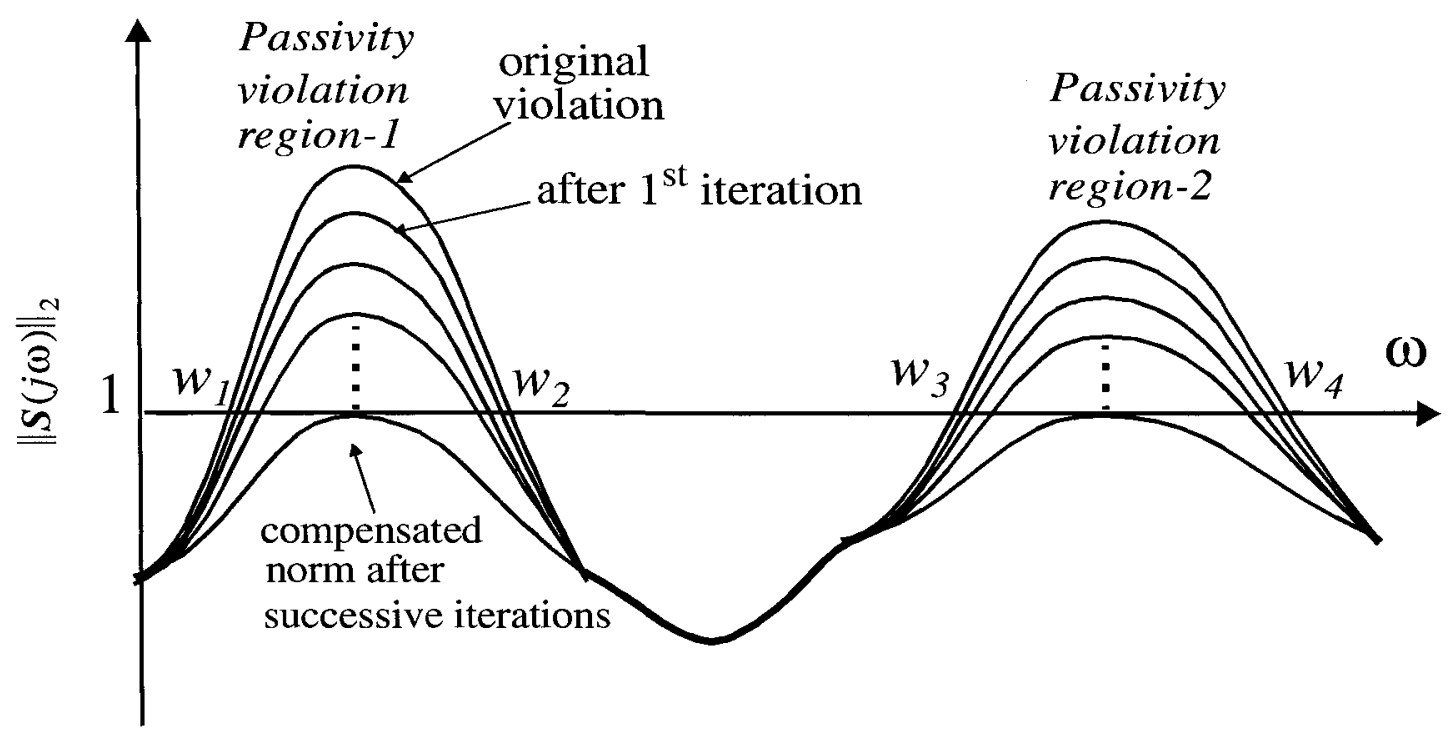

Fig. 5.3. Norm-spectrum with multiple regions of violation and compensation by iterative perturbation of matrix $\mathbf{C}$

The advantage of the proposed fast-sweep based passivity verification and compensation process is that, it has been accomplished without the need for any formulation of computationally expensive Hamiltonian matrices and evaluation of their imaginary eigenvalues/eigenvectors during any of the intermediate iterations.

Once the proposed algorithm confirms/generates a passive macromodel, a Hamiltonian based check of the macromodel is performed using state-of-the-art methods [54], [55] at the exit point of the proposed algorithm. Hence the requirement for use of Hamiltonian matrices is kept to the minimum, leading to reduced computational time.

A summary of the steps involved in the proposed passivity enforcement algorithm is given in Fig. 5.4. 
- Step 1: Obtain multiport S-parameter tabulated data up to $f_{\max }$ (maximum frequency of interest).

- Step 2: Compute the multiport pole-residue model (5.1). Obtain the state-space system $(\boldsymbol{A}, \boldsymbol{B}, \boldsymbol{C}, \boldsymbol{D})(5.2)$.

- Step 3: Compute the reciprocal system $\left(\boldsymbol{A}_{r}, \boldsymbol{B}_{r}, \boldsymbol{C}_{r}, \boldsymbol{D}_{r}\right)(5.10)$.

- Step 4: Perform the frequency sweep of the norm of the scattering matrix using the closed-form of (5.1) and find the regions of passivity violation up to $f_{\text {max }}$. Beyond $f_{\text {max }}$, find the passivity violation regions by performing the frequency sweep of the reciprocal system (5.16) in its pole-residue form.

if the macromodel is non-passive:

- Step 5: Perform the passivity compensation, by solving (5.17) at the frequency points of maximum violation. Obtain the updated residue matrix $C$. Go to Step 2.

Else stop.

Fig. 5.4. Pseudocode for the proposed passive macromodeling algorithm for large scale systems

\subsection{Numerical Results}

In this section, two numerical examples are presented to demonstrate the validity and efficiency of the proposed algorithm. The first example is a relatively small system and the second example corresponds to a system with a high order macromodel. The CPU times reported here correspond to a PC platform with $1 \mathrm{~GB}$ RAM and 2GHz AMD processor. 


\subsubsection{Example 1: IC Package}

In this example, the tabulated $S$-parameter data of a 14-port IC package structure up to $6 \mathrm{GHz}$ is considered (supplied by the CST GmBH, Germany). The data was modeled with a rational function approximation using a common set of 14 poles. This resulted in a state-space system of order $14 \times 14=196$. The resulting macromodel is found to be non-passive as illustrated in Fig. 5.5, which shows frequency sweep of the 2-norm of the scattering matrix versus frequency.

The usefulness of employing a reciprocal system for identifying the passivity violation beyond $f_{\max }$ is demonstrated in Fig. 5.6. Here the passivity violation region beyond $f_{\max }$ is well identified by the reciprocal system. The proposed closed-form frequency-sweep method was significantly faster requiring only 0.001 seconds for sweep at 1000 points of both original and reciprocal systems.

This is a relatively small macromodel and can also be practically handled by the Hamiltonian based verification methods. The above results were verified using the Hamiltonian based passivity check and identification of violating regions as outlined in the Section 4.2 .3 and it required 0.9 seconds.

Next, the impact of the passivity checking methodologies on passivity compensation process was investigated. For this purpose, the passivity compensation based on first-order residue perturbation (PCFOP) (of Section 4.2.4a) was performed using both types of passivity verification methods: proposed closed-form frequency-sweep (of Section 5.2.1) and Hamiltonian based methods (of Section 4.2.3). The passivity compensation in conjunction with the proposed frequency sweep required only 25 seconds. On the other hand, passivity compensation with Hamiltonian based verification required 64 seconds. 
Fig. 5.7 shows sample responses of the original data compared with the macromodel before and after compensation. As seen, they match accurately. The relative norm of the perturbation of the residue matrix $\|\Delta C\|_{F} /\|C\|_{F}$ for this example is $1.1587 \times 10^{-7}$.

It should be noted that there was no significant difference in CPU time required for passivity compensation when the PCFOP was replaced by the compensation algorithm based on linear matrix inequality (PCLMI - Section 4.2.4b) during the above analysis.

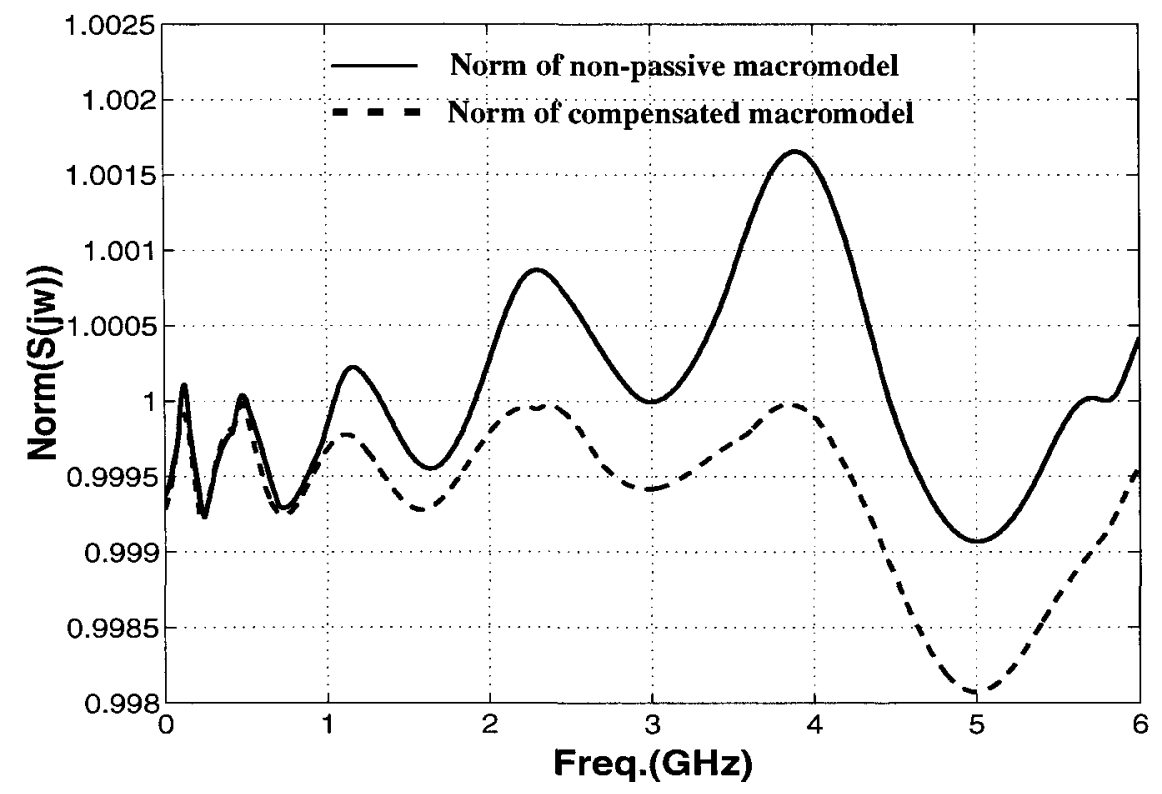

Fig. 5.5. Comparison of norm of $S(j \omega)$ : IC package 

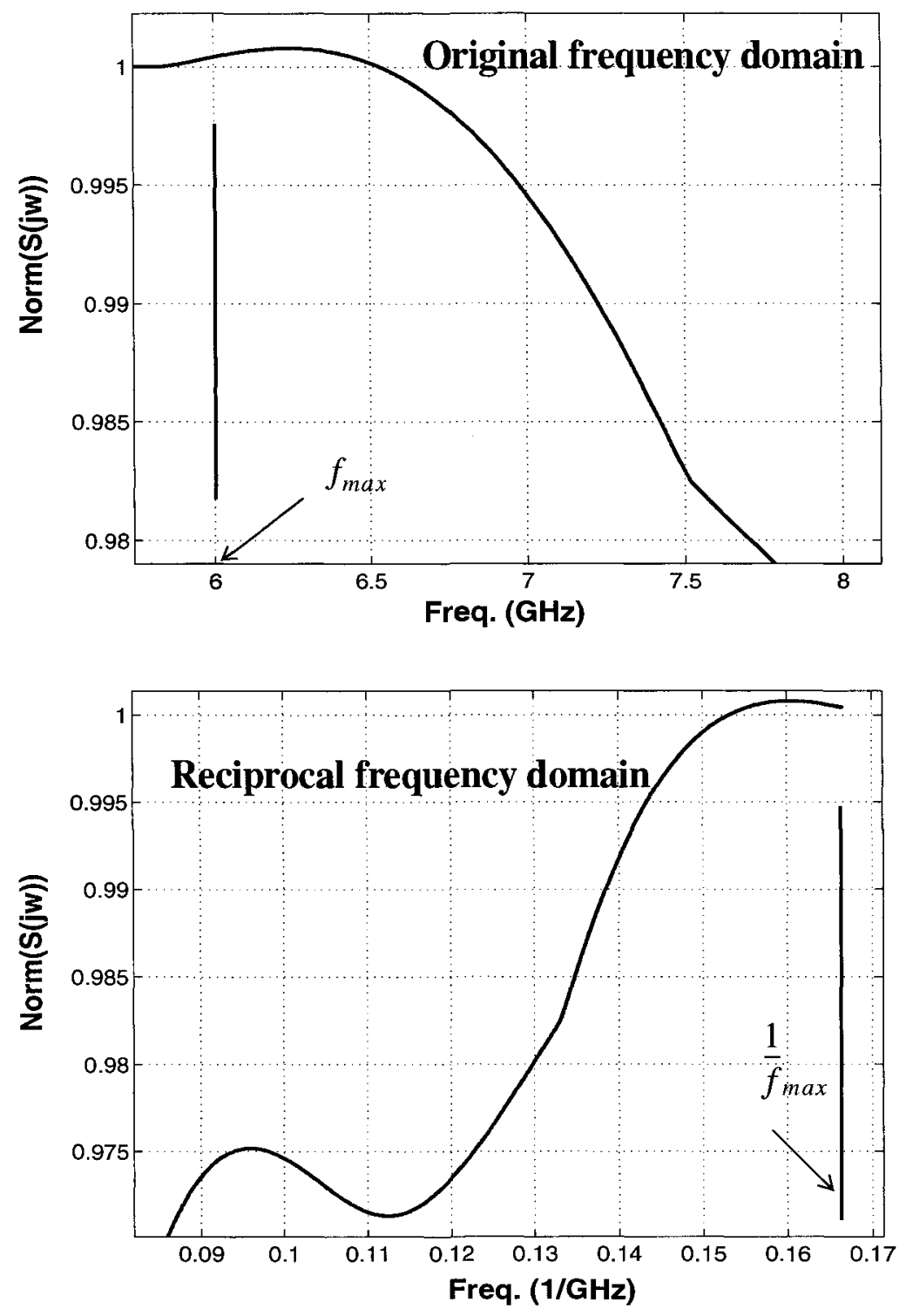

Fig. 5.6. Identification of passivity violation beyond $f_{\max }$ 


\section{Original data}

- - Response before compensation

- - Response after compensation
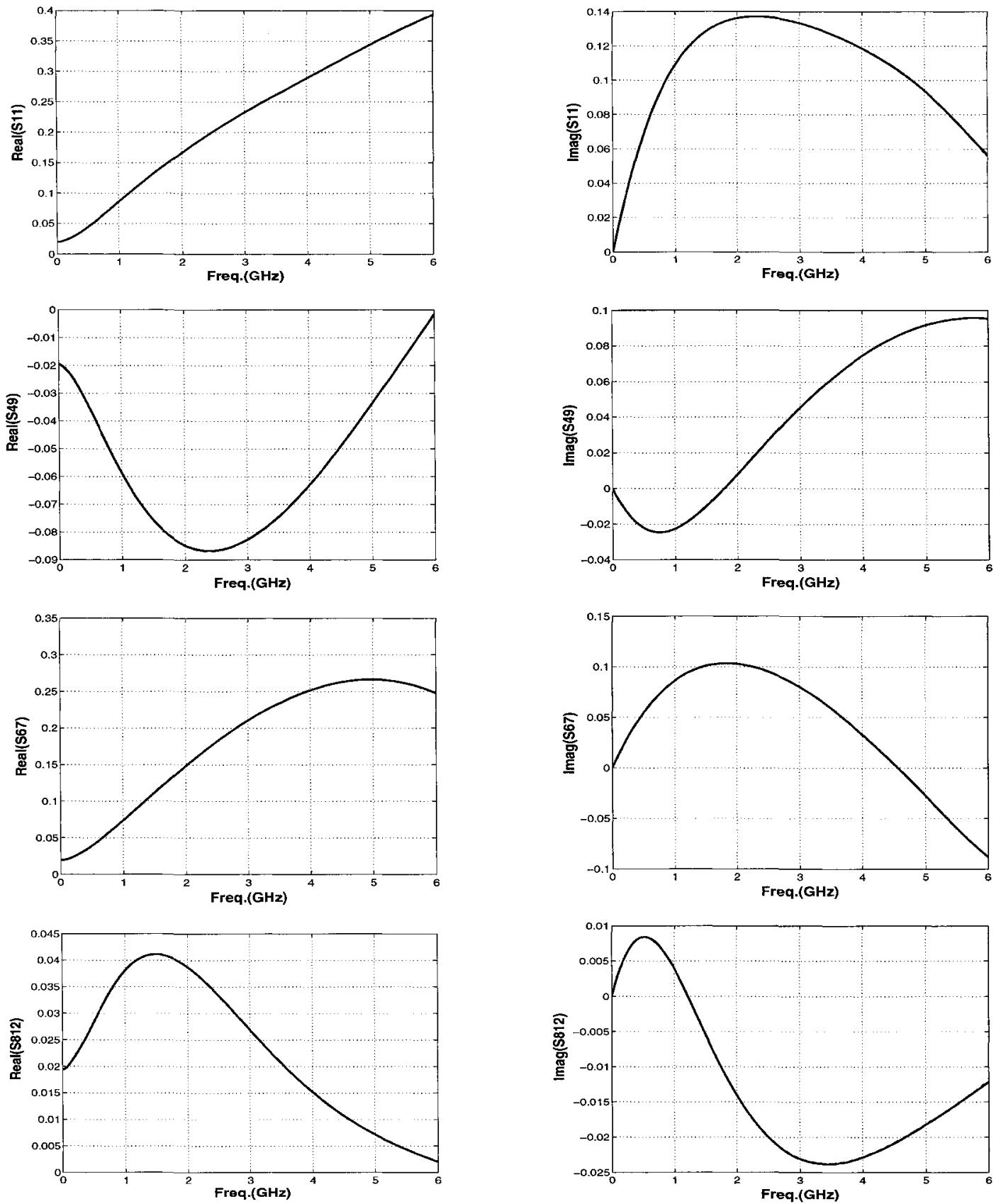

Fig. 5.7. Comparison of sample S-parameters: IC package 


\subsubsection{Example 2: 12-Port Network}

In this example, the state-space macromodel representing the measured data of a 12-port network, provided by the Sigrity Inc., CA, USA was considered. The macromodel was nonpassive as evident in Fig. 5.8. The size of state-space system was 1488, which is relatively large. Consequently, for this example, a considerable $\mathrm{CPU}$ speedup was achieved during the passivity compensation, by employing the proposed fast passivity verification (Section 5.2 .1 ) and the efficient solution of Lyapunov equation (Section 5.3.1a).

For comparison purposes, the CPU times for passivity verification using both the Hamiltonian and proposed methods were investigated. Since the size of the statespace system is large, the direct eigenvalue solution of the Hamiltonian matrix takes a very long time (16 minutes for a full eigenvalue computation), which is required during each iteration. Hence, when using the Hamiltonian based passivity checking procedure outlined in the Section 4.2.3, the algorithm described in [55] was used to accelerate the detection of imaginary eigenvalues of the Hamiltonian matrix. Passivity compensation (PCFOP) using Hamiltonian based verification (in conjunction with the accelerated identification of imaginary eigenvalues) required 7.9 minutes.

On the other hand, when the passivity compensation is performed in conjunction with the proposed fast frequency sweep based verification, it required only 4.63 minutes, giving a significant speedup.

It is to be noted that, in both the above reported results, considerable CPU time was saved by using the proposed solution of Lyapunov equation (described in Section 5.3.1a) as compared to the conventional full solution (function lyap of MATLAB). The full solver took 14.3 minutes, whereas the proposed Lyapunov 
equation solution (which exploits the canonical form of the macromodel) required only 4 seconds.

Table 5.2 and Table 5.3 summarize the comparison of CPU times for the proposed PCFOP passivity enforcement in conjunction with the new verification algorithm as well as traditional Hamiltonian based methods. The relative norm of the perturbation of the residue matrix $\|\Delta C\|_{F} /\|C\|_{F}$ for this example was $3.4 \times 10^{-4}$. Passivity compensation using both the proposed and Hamiltonian based verifications required 11 iterations. It should be noted that, for broadband largeorder multiport macromodels with many regions of passivity violations (and also larger violations), the number of iterations required during the compensation process can be very high, leading to higher speed-ups using the proposed algorithm.

Norm comparison of the macromodel before and after compensation is shown in Fig. 5.8. Accuracy comparison of some sample responses of this system are shown in Fig. 5.9, and as seen, they match accurately. 
Table. 5.2. Summary of CPU times for passivity enforcement using PCFOP algorithm in conjunction with different verification methods and full Lyapunov solution - example 2

\begin{tabular}{|l|c|}
\hline \multicolumn{1}{|c|}{$\begin{array}{c}\text { Passivity verification method used } \\
\text { (during each iteration) }\end{array}$} & $\begin{array}{c}\text { Total CPU time } \\
\text { (Verification+ Full Lyapunov solution + } \\
\text { PCFOP) }\end{array}$ \\
\hline \hline $\begin{array}{l}\text { Hamiltonian based passivity checking } \\
\text { (Section } 4.2 .3+[55] \text { ) }\end{array}$ & 22.2 minutes \\
\hline $\begin{array}{l}\text { Proposed fast passivity checking } \\
\text { (Section 5.2.1) }\end{array}$ & 18.9 minutes \\
\hline
\end{tabular}

Table. 5.3. Summary of CPU times for passivity enforcement using PCFOP algorithm in conjunction with different verification methods and proposed fast Lyapunov solution - example 2

\begin{tabular}{|l|c|}
\hline \multicolumn{1}{|c|}{$\begin{array}{c}\text { Passivity verification method used } \\
\text { (during each iteration) }\end{array}$} & $\begin{array}{c}\text { Total CPU time } \\
\text { (Verification+ Fast Lyapunov } \\
\text { solution + PCFOP) }\end{array}$ \\
\hline \hline $\begin{array}{l}\text { Hamiltonian based passivity checking } \\
\text { (Section } 4.2 .3+[55] \text { ) }\end{array}$ & 7.9 minutes \\
\hline $\begin{array}{l}\text { Proposed fast passivity checking } \\
\text { (Section 5.2.1) }\end{array}$ & 4.63 minutes \\
\hline $\begin{array}{l}\text { Proposed fast passivity checking } \\
\text { (Section 5.2.1) } \\
\text { + Final Hamiltonian based check ([55]) }\end{array}$ & 4.93 minutes \\
\hline
\end{tabular}




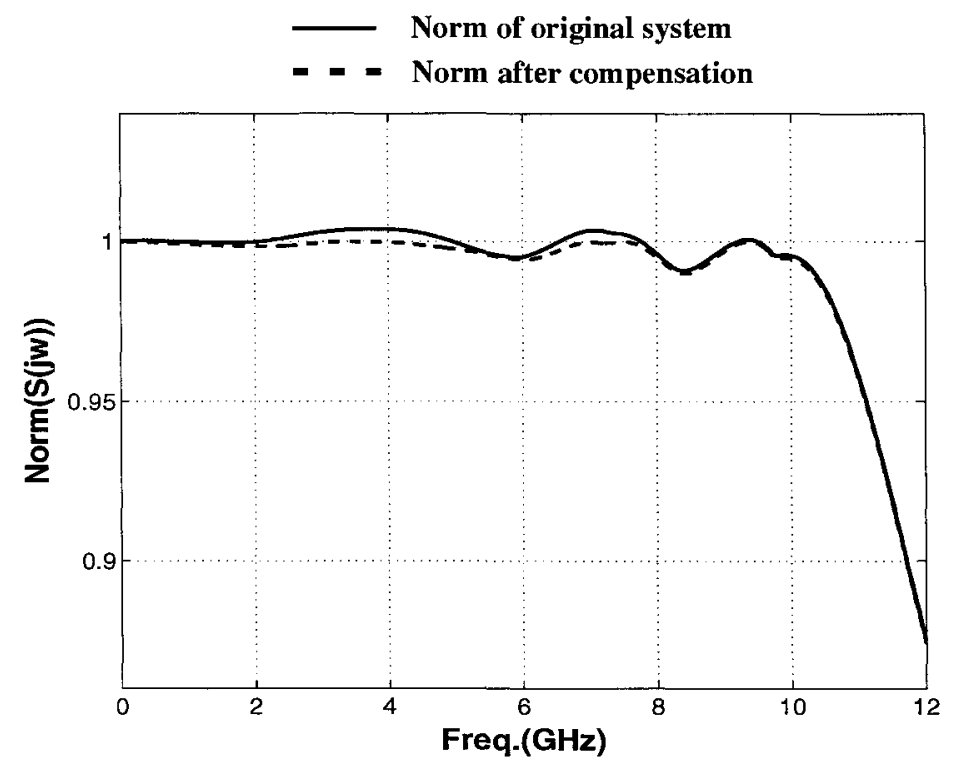

Fig. 5.8. Comparison of norm of $S(j \omega)$ : example 2

Next, a comparison of the above analyses was done by replacing the PCFOP with the passivity compensation algorithm based on linear matrix inequality (PCLMI Section $4.2 .4 \mathrm{~b})$. The CPU times in this case were comparable to the previous analysis. 
Response before compensation

- - Response after compensation
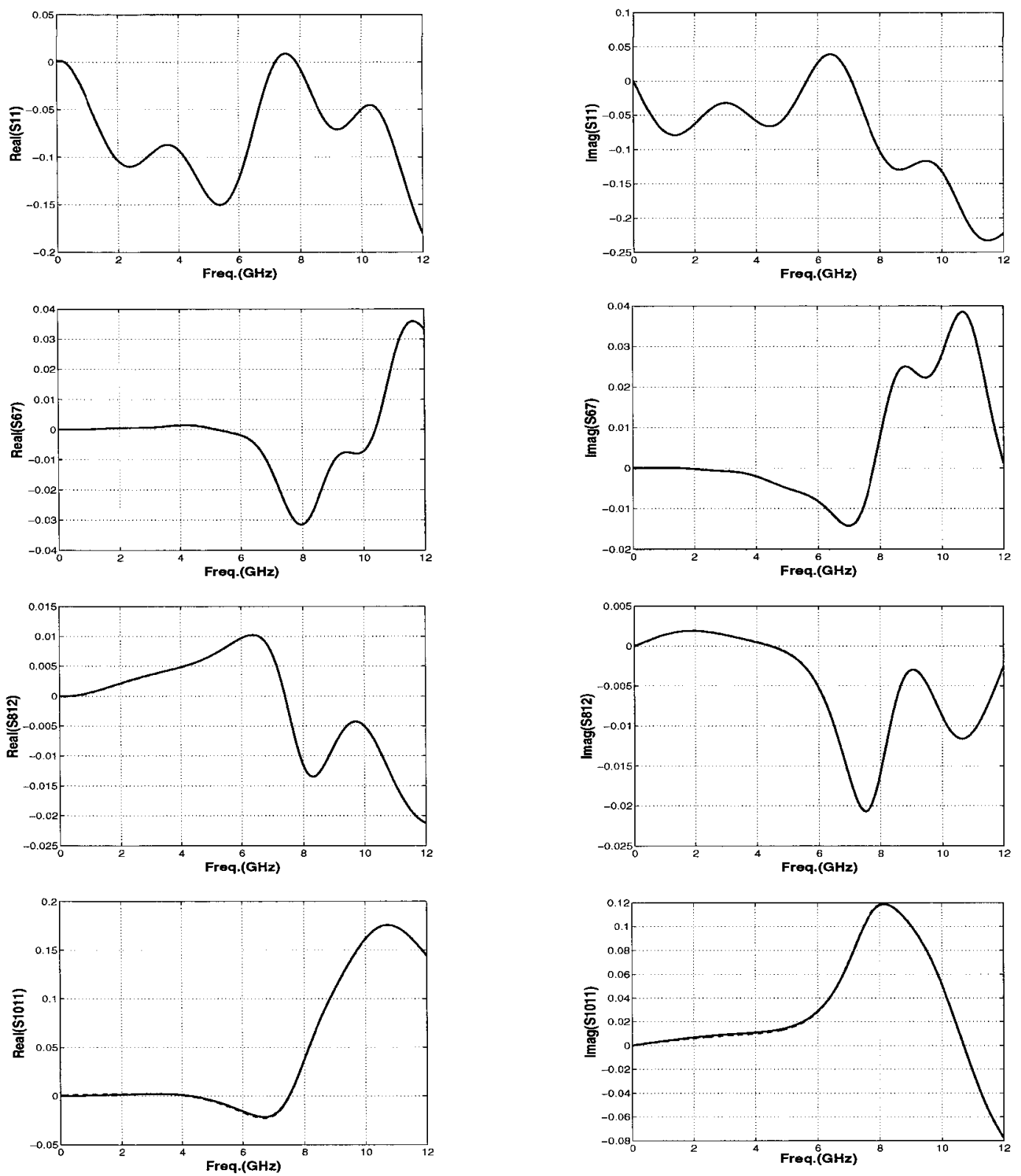

Fig. 5.9. S-parameters of example 2 


\subsection{Conclusion}

In this chapter, a fast technique is presented for passive macromodeling of subnetworks characterized by $S$-parameter tabulated data with large-order macromodels. It is based on the fast frequency sweep of the response. For this purpose, a reciprocal system formulation has been proposed for covering the entire frequency range $(0-\infty)$ for the sweeping purpose. The advantage of this technique is that, the number of Hamiltonian eigen evaluations during the iterative passivity compensation process of large-order macromodels is minimized, resulting in computational speed-up. In addition, a method for fast solution of Lyapunov equation of the state-space system is presented, which is required by passivity enforcement algorithms to maintain the macromodel accuracy during the compensation process.

One of the issues with the iterative algorithms for passive macromodeling described in this chapter, chapter 4 and in [43] is that, the passivity compensation process does not have a guaranteed direction for correction. In other words, the passivity compensation at one frequency point may lead to passivity violation at some other frequency points, which in turn will have to be corrected for passivity. This phenomenon may ultimately increase the number of iterations for passivity enforcement leading to excessive CPU time. In order to overcome this issue, an algorithm with a guaranteed search direction of passivity enforcement is presented in the next chapter. 


\section{CHAPTER 6 Global Passivity Enforcement Algorithm for Macromodels of Tabulated Data}

As discussed in chapter 4 and 5, several iterative algorithms have been proposed for the passivity enforcement of nonpassive macromodels of tabulated networks [39] - [43], [49] - [55], suitable for both small and large order macromodels. However, one of the issues still of concern in these algorithms is that, the passivity correction process does not have a guaranteed direction. In other words, the passivity compensation at one frequency point may lead to passivity violation at some other frequency points, which in turn will have to be corrected for passivity. This increases the number of iterations required for the complete passivity enforcement process and thereby the CPU cost. In order to address this problem, a global passivity enforcing algorithm is presented in this chapter. The algorithm employs a guaranteed search direction for enforcing passivity, such that the correction for passivity at a certain frequency region doesn $t$ introduce new regions of passivity violation. This algorithm is very efficient for small passivity violations and has been developed for macromodels of tabulated data in admittance form. 


\subsection{Problem Formulation}

The admittance $(Y)$ matrix of a $m$-port subnetwork can be written in terms of a pole-residue formulation as

$$
\boldsymbol{Y}(s)=\left[Y_{i j}(s)\right] ; \quad Y_{i j}(s)=\sum_{i=1}^{n} \frac{k_{i}^{i, j}}{S-p_{i}}+d^{i, j} ; \quad(i, j \in 1 \ldots m)
$$

where the residues $\left(k_{i}\right)$ and poles $\left(p_{i}\right)$ can be real or complex conjugate pairs, $n$ is the total number of poles and $d$ represents the direct coupling constant. Next, the state-space representation for (6.1) can be obtained as [80], [85], [86]

$$
\begin{aligned}
\dot{\boldsymbol{x}}(t) & =\boldsymbol{A} \boldsymbol{x}(t)+\boldsymbol{B u}(t) \\
\boldsymbol{y}(t) & =\boldsymbol{C} \boldsymbol{x}(t)+\boldsymbol{D u}(t)
\end{aligned}
$$

where $\boldsymbol{x}(t) \in \mathfrak{R}^{q \times 1}$ (with $q=n m$ ) is the state vector, $\boldsymbol{u}(t) \in \mathfrak{R}^{m \times 1}$ is the input vector. $\boldsymbol{A} \in \mathfrak{R}^{q \times q}$ is the state-matrix, $\boldsymbol{B} \in \mathfrak{R}^{q \times m}$ is the matrix that relates the inputs to statevariables, $C \in \mathfrak{R}^{m \times q}$ is the matrix relating state variables to the outputs $\left(\boldsymbol{y}(t) \in \Re^{m \times 1}\right)$ and $\boldsymbol{D} \in \Re^{m \times m}$ is a matrix of direct coupling constants. The relationship between the input $\boldsymbol{u}(s)$ and output $\boldsymbol{y}(s)$ in frequency domain can be written as

$$
\boldsymbol{Y}(s)=\boldsymbol{C}(s \boldsymbol{I}-\boldsymbol{A})^{-1} \boldsymbol{B}+\boldsymbol{D}
$$

The conditions for a network with admittance matrix $\boldsymbol{Y}(s)$ to be passive are [67], [69], [85], [86]:

(a) $\boldsymbol{Y}\left(s^{*}\right)=\boldsymbol{Y}^{*}(s)$, where ${ }^{*}$ is the complex conjugate operator. 
(b) All elements of $\boldsymbol{Y}(s)$ are analytic in $\operatorname{Re}(s)>0$

(c) $\boldsymbol{Y}(s)$ is a positive-real (PR) matrix, i.e., the product $z^{* T}\left[\boldsymbol{Y}^{T}\left(s^{*}\right)+\boldsymbol{Y}(s)\right] z \geq 0$ for all complex values of $s$ with $\operatorname{Re}(s)>0$ and any arbitrary vector $z$.

For the case of rational functions in $s$ having no pure imaginary poles, condition (c) simplifies to [69]

$$
z^{* T}\left[\boldsymbol{Y}^{T}\left(j \omega^{*}\right)+\boldsymbol{Y}(j \omega)\right] z \geq 0 ; \quad \text { for } \omega \in \Re \cup \infty
$$

Condition (a) is automatically satisfied since the complex poles/residues of the transfer function are always considered along with their conjugates, leading to only real coefficients in rational functions of $\boldsymbol{Y}(s)$. Condition (b) implies that there are no unstable poles in any element of the matrix $\boldsymbol{Y}(s)$, and can be readily satisfied during the approximation process. However, ensuring condition (c) is not easy. For the practical case of networks with symmetric admittance matrices, condition (c) can also be expressed using (6.3) and (6.4) as:

$$
\operatorname{Real}(\boldsymbol{Y}(j \omega))=\boldsymbol{F}(j \omega)=-\boldsymbol{C A}\left(\omega^{2} \boldsymbol{I}+\boldsymbol{A}^{2}\right)^{-1} \boldsymbol{B}+\boldsymbol{D} \geq 0, \text { for } \omega \in \Re \cup \infty
$$

Condition (6.5) also implies that all the eigenvalues of $\boldsymbol{F}(j \omega)$ must be greater than or equal zero for $\omega \in \mathfrak{R} \cup \infty$. In the subsequent sections of this chapter, a new passivity verification and compensation algorithm with a guaranteed search direction is presented. 


\subsection{Passivity Verification}

For the case of Y-parameter based macromodels, the following two theorems from the control area are used (on similar lines to that of scattering parameters) for systematic passivity verification without resorting to frequency-sweep.

Theorem 1: The state-space system $(\boldsymbol{A}, \boldsymbol{B}, \boldsymbol{C}, \boldsymbol{D})$ is passive iff the following Hamiltonian matrix $(M)[70]$ has no imaginary eigenvalues.

$$
\boldsymbol{M}=\left[\begin{array}{cc}
\boldsymbol{A}-\boldsymbol{B}\left(\boldsymbol{D}+\boldsymbol{D}^{T}\right)^{-1} \boldsymbol{C} & \boldsymbol{B}\left(\boldsymbol{D}+\boldsymbol{D}^{T}\right)^{-1} \boldsymbol{B}^{T} \\
-C^{T}\left(\boldsymbol{D}+\boldsymbol{D}^{T}\right)^{-1} C & -\boldsymbol{A}^{T}+C^{T}\left(D+D^{T}\right)^{-1} \boldsymbol{B}^{T}
\end{array}\right]
$$

If the macromodel is nonpassive, it needs to be corrected (compensated for passivity violation). For a successful and fast compensation, it is essential to know the exact frequency locations at which eigenvalues of $\boldsymbol{F}(j \omega)$ cross over from positive value to negative value. For this purpose the following theorem [28], [39], [40], [87] is used.

Theorem 2: The real part of the symmetric admittance matrix, $\boldsymbol{F}\left(j \omega_{0}\right)$, is singular if $j \omega_{0}$ is an eigenvalue of the corresponding Hamiltonian matrix $\boldsymbol{M}$, provided $\boldsymbol{D}+\boldsymbol{D}^{T}$ is a positive definite matrix.

Theorem-2 implies that an imaginary eigenvalue of the Hamiltonian matrix $\boldsymbol{M}$ corresponds to the frequency at which $\boldsymbol{F}(j \omega)$ becomes singular (i.e. the macromodel becomes non-passive). This information of exact locations where an eigenvalue of $\boldsymbol{F}(j \omega)$ becomes zero is very crucial as its knowledge helps the 
passivity compensation process. The proof of Theorem- 2 and its corollary is given in Appendix $C$. The next section describes a new passivity compensation (correction) algorithm with guaranteed search direction. It is assumed that the macromodel is asymptotically passive at $\omega=\infty$ (i.e. $\boldsymbol{D}+\boldsymbol{D}^{T}>0$ ), which can be easily ensured using algorithms such as [26], [28].

\subsection{Passivity Enforcement}

As discussed in the introduction, several algorithms [39] - [43], [49] - [55] were recently proposed to enforce passivity in macromodels with small violations. But, these algorithms suffer from the limitation that the passivity violation may be introduced at some other frequency points while performing correction in a certain frequency region. In order to overcome this difficulty, a new algorithm [56] employing a guaranteed search direction for enforcing passivity, such that the correction for passivity in a certain frequency region doesn $\mathrm{t}$ introduce new regions of passivity violation, is presented in this section. The proposed method performs compensation by refining only few selected elements of the residue matrix $C$ of the state-space system. These elements are selected from the real part of residues of diagonal elements of $\boldsymbol{Y}(s)$, corresponding to the poles in the vicinity of passivity violation. The proposed passivity enforcement algorithm consists of the following three steps:

Determination of passivity violation regions.

Determination of magnitude of the maximum violation in a given non-passive region.

Performing passivity correction by perturbing the selected residues.

The details of these steps are given below. 


\subsubsection{Determination of Passivity Violation Regions}

This step is similar to that of the one described for scattering parameters in Section 4.2.3, with the exception that the explicit relations are developed for the case of $Y$-parameters. It is known from Theorem-2 that the imaginary eigenvalues of the Hamiltonian matrix $\boldsymbol{M}$ of (6.6) correspond to the frequency locations where $\boldsymbol{F}(j \omega)$ becomes singular. However, this information does not convey anything about the regions of passivity violation (i.e. the frequency bandwidth in which an eigenvalue of $\boldsymbol{F}(j \omega)$ is negative). In order to determine the regions of passivity violation, the slope of eigenvalues of $\boldsymbol{F}(j \omega)$ at its singular locations is determined. This is done as follows.

If $\lambda$ is an eigenvalue of $\boldsymbol{F}(j \omega)$ and $\boldsymbol{u}$ the corresponding right eigenvector, then

$$
(\boldsymbol{F}(j \omega)-\lambda \boldsymbol{I}) \boldsymbol{u}=0
$$

Differentiating the above equation with respect to the angular frequency $\omega$, we get

$$
\left(\frac{d \boldsymbol{F}(j \omega)}{d \omega}-\frac{d \lambda}{d \omega} \boldsymbol{I}\right) \boldsymbol{u}+(\boldsymbol{F}(j \omega)-\lambda \boldsymbol{I}) \frac{d \boldsymbol{u}}{d \omega}=0
$$

Next, multiplying (6.8) by the left eigenvector $\left(v^{T}\right)$ of $\boldsymbol{F}(j \omega)$

$$
v^{T} \frac{d \boldsymbol{F}(j \omega)}{d \omega} \boldsymbol{u}-\boldsymbol{v}^{T} \frac{d \lambda}{d \omega} \boldsymbol{u}+\boldsymbol{v}^{T} \underbrace{(\boldsymbol{F}(j \omega)-\lambda \boldsymbol{I}) \frac{d \boldsymbol{u}}{d \omega}}_{=0}=0
$$

Notice that the last term on the left hand side of (6.9) is zero by the definition of left eigenvector. Using this fact, (6.9) can be rewritten as 


$$
\boldsymbol{v}^{T} \frac{d \lambda}{d \omega} \boldsymbol{u}=\boldsymbol{v}^{T} \frac{d \boldsymbol{F}(j \omega)}{d \omega} \boldsymbol{u}
$$

or

$$
\frac{d \lambda}{d \omega}=\frac{v^{T} \frac{d F(j \omega)}{d \omega} u}{v^{T} u}
$$

Next, derivative of $\boldsymbol{F}(j \omega)$ with respect to $\omega$ can be obtained using (6.5) as

$$
\frac{d \boldsymbol{F}(j \omega)}{d \omega}=\boldsymbol{C A}\left(\omega^{2} \boldsymbol{I}+\boldsymbol{A}^{2}\right)^{-2} 2 \omega \boldsymbol{B}
$$

Substituting (6.12) into (6.11) we get

$$
\frac{d \lambda}{d \omega}=\frac{\boldsymbol{v}^{T}\left(\boldsymbol{C A}\left(\omega^{2} \boldsymbol{I}+\boldsymbol{A}^{2}\right)^{-2} 2 \omega \boldsymbol{B}\right) \boldsymbol{u}}{\boldsymbol{v}^{T} \boldsymbol{u}}
$$

Using (6.13) the slope of an eigenvalue of $\boldsymbol{F}(j \omega)$ at its singular frequency can be determined. The regions and bandwidth of local passivity violation are then determined using the following steps:

- Step 1: Collect the pure imaginary eigenvalues (consider only those with positive imaginary parts) of the Hamiltonian matrix $\boldsymbol{M}$ of (6.6) in a vector $\boldsymbol{S}_{a}=\left[\omega_{1}, \omega_{2} \ldots \omega_{T}\right]$, such that $\omega_{1}<\omega_{2}<\ldots<\omega_{T}$, where ' $T$ ' is the total number of such entries. Let $\omega_{H}=\omega_{T}$.

It should be noted that, finding the imaginary eigenvalue may not be a trivial task, since the real part of the eigenvalue may not be exactly zero owing to the presence of numerical noise. In this dissertation, this difficulty is overcome by using the property of the Hamiltonian matrix, that, its eigenvalue spectrum is symmetric with reference to both real as well as imaginary axis. This implies that, if $\lambda$ is a complex eigenvalue of a given Hamiltonian matrix, then $-\lambda, \lambda^{*},-\lambda^{*}$ are 
also its eigenvalues. On the other hand, the imaginary eigenvalues are symmetric only with respect to the real axis (i. e., if $\lambda$ is an imaginary eigenvalue of a given Hamiltonian matrix, then $-\lambda$ is also its eigenvalue). As a consequence of this property, while determining if an eigenvalue is imaginary or not, we check for the eigenvalues which are symmetric only about the real axis. As a result, the effect of numerical noise is taken care of while identifying the imaginary eigenvalues of the Hamiltonian matrix.

- Step 2: Next, at the frequency corresponding to each of the above entries, evaluate the slope of the eigenvalue of $F(j \omega)$ using (6.13). Note that the slope at $\omega_{T}$ is always positive since $\mathrm{D}+\mathrm{D}^{T}>0$.

- Step 3: Count the number of positive and negative slopes starting from $\omega_{H}$. When the count of positive and negative slopes become equal, say at $\omega_{k}$, then the first region of local passivity violation is established (i.e. the region between $\omega_{H}$ to $\left.\omega_{k}\right)$.

- Step 4: Reset the count of slope to zero and designate $\omega_{H}=\omega_{k-1}$ and repeat steps 3 and 4 until all entries in the vector $S_{a}$ are exhausted.

The above steps are illustrated in the Fig. 6.1(a), for the case of Hamiltonian matrix having six pairs of imaginary eigenvalues.

The situation when the passivity violation starts at zero frequency is illustrated with an example in Fig. 6.1(b). As seen, in this example, when the counting of slopes is restarted after determining the second region of violation, the number of positive slopes becomes one and the number of negative slopes is equal to zero, at the end of counting the slopes. In such cases, a region of violation exists between the first frequency point from where the counting of slope is restarted (in this case $\left.\omega_{1}\right)$ and zero Hertz (origin). 
The above proposed passivity enforcement algorithm assumes that the imaginary eigenvalues of the Hamiltonian matrix are simple. However, it can be easily extended to the case of Hamiltonian matrices with repeated imaginary eigenvalues using the well established approach outlined in [43].

(a)

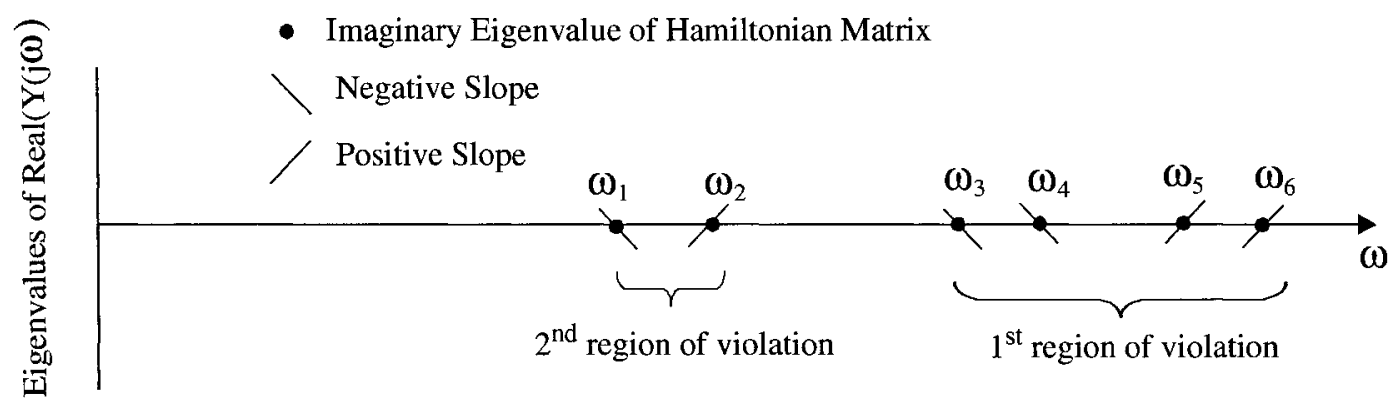

(b)

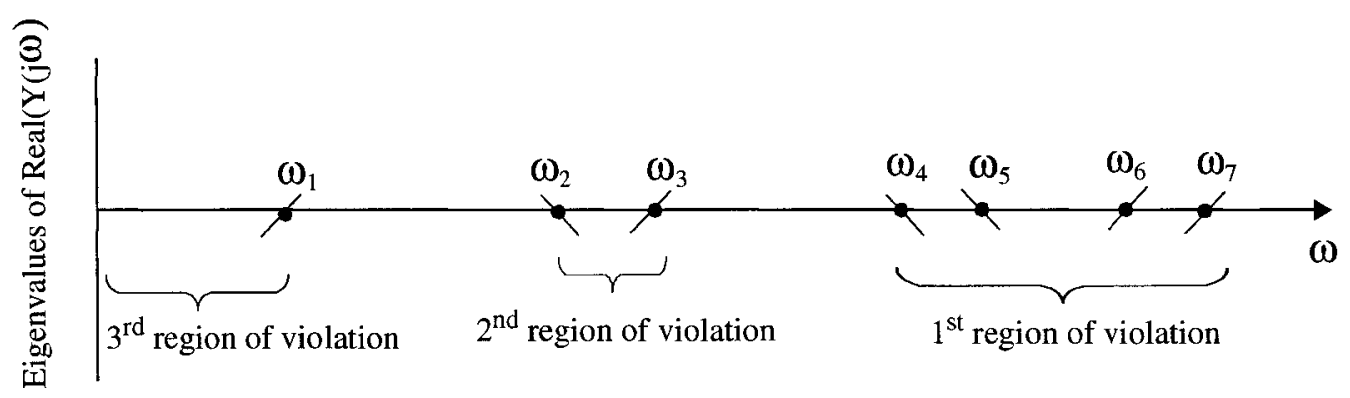

Fig. 6.1. Illustration of identification of regions of passivity violation

Subsequently, the exact location of maximum passivity violation (i.e. the maximum negative eigenvalue of $\boldsymbol{F}(j \omega))$ is determined in each region of passivity violation. This location corresponds to the maximum negative eigenvalue of $\boldsymbol{F}(j \omega)$. These locations are found by solving the following problem in each region of passivity violation.

$$
\min \operatorname{eig}(\operatorname{Real}(Y(j \omega))) ; \quad \omega_{l} \leq \omega \leq \omega_{h}
$$


where $\omega_{l}, \omega_{h}$ are the boundaries of a passivity violating region. For instance, in the first region of violation in Fig. 6.1(a), $\omega_{l}=\omega_{3}$ and $\omega_{h}=\omega_{6}$. The problem in (6.14) converges very fast as it is associated with only one variable $(\omega)$ and has a good initial guess (midpoint of $\omega_{l}$ and $\omega_{h}$ ). These locations are addressed first during the compensation process.

\subsubsection{Passivity Enforcement with Guaranteed Search Direction}

With the regions of passivity violation as well as the location and magnitude of maximum violation in each such regions known, the passivity compensation is performed as follows. Consider the real part of the admittance matrix, given by (6.5). If the macromodel is non-passive (i.e. $\boldsymbol{F}(j \omega)$ is negative definite), the residue matrix $\boldsymbol{C}$ is perturbed by $\Delta \boldsymbol{C}$ (keeping matrices $\boldsymbol{A}, \boldsymbol{B}$ and $\boldsymbol{D}$ unchanged) so that

$$
\boldsymbol{F}(j \omega)+\Delta \boldsymbol{F}(j \omega)=-(\boldsymbol{C}+\Delta \boldsymbol{C}) \boldsymbol{A}\left(\omega^{2} \boldsymbol{I}+\boldsymbol{A}^{2}\right)^{-1} \boldsymbol{B}+\boldsymbol{D} \geq 0
$$

at the frequency point of maximum violation, in a non-passive region under consideration. For instance, let $-\Delta \lambda$ represent the eigenvalue of $\boldsymbol{F}(j \omega)$ at a frequency point of maximum passivity violation. To compensate this, $\Delta \boldsymbol{F}(j \omega)=-\Delta \boldsymbol{C A}\left(\omega^{2} \boldsymbol{I}+\boldsymbol{A}^{2}\right)^{-1} \boldsymbol{B}$ is added to $\boldsymbol{F}(j \omega)$ by slightly perturbing the matrix $C$, such that [82]

$$
\Delta \lambda=\frac{\boldsymbol{y}^{T} \Delta \boldsymbol{F}(j \omega) \boldsymbol{x}}{\boldsymbol{y}^{T} \boldsymbol{x}}=\frac{-\boldsymbol{y}^{T} \Delta \boldsymbol{C A}\left(\omega^{2} \boldsymbol{I}+\boldsymbol{A}^{2}\right)^{-1} \boldsymbol{B} \boldsymbol{x}}{\boldsymbol{y}^{T} \boldsymbol{x}}
$$

where $\boldsymbol{y}$ and $\boldsymbol{x}$ are the left and right eigenvectors of $\boldsymbol{F}(j \omega)$ respectively. After some algebraic manipulations, (6.16) can be converted into a set of linear equations of the form: 


$$
\Delta \lambda=\Theta Q
$$

where, the unknown perturbed values of selected residues are contained in the vector $Q$. Next, the details of the selection of appropriate residues for perturbation, to achieve desired correction is given.

\subsection{2a Selection of Appropriate Residues for Perturbation}

In the proposed algorithm, the perturbation is effected on real-part of residues of driving point admittances corresponding to the poles in the vicinity of passivity violation. Identification of such poles is done by determining the contribution of each pole to the real part of diagonal elements of the admittance matrix, in the frequency region of violation. This is done by integrating the square of the real part of the response (represented by $R(\omega)$ ) of every pole for a passivity violating region under consideration (with boundaries $\omega_{1}$ and $\omega_{2}$ ),

$$
\int_{\omega_{1}}^{\omega_{2}}(R(\omega))^{2} d \omega
$$

The poles with the significant contribution are selected, and their residues corresponding to the driving point admittances are perturbed during passivity compensation process. Next, an appropriate mapping of such residues to the residue matrix $C$ (for formulating $\Delta C$ and $Q$ of (6.15) and (6.17), respectively) is illustrated using the following example.

Consider a two port network with two poles $p_{1}=w+j z, p_{2}=w-j z$. Let the corresponding residues at different ports be $c_{k, l}=(r \pm j g)_{k, l} ; \quad k, l=1,2$. The statespace realization [78] - [80] for the network can be expressed as, 


$$
\begin{aligned}
& {\left[\begin{array}{l}
\dot{x}_{1} \\
\dot{x}_{2} \\
\dot{x}_{3} \\
\dot{x}_{4}
\end{array}\right]=\underbrace{\left[\begin{array}{cccc}
w & 0 & z & 0 \\
0 & w & 0 & z \\
-z & 0 & w & 0 \\
0 & -z & 0 & w
\end{array}\right]}_{\boldsymbol{A}}\left[\begin{array}{l}
x_{1} \\
x_{2} \\
x_{3} \\
x_{4}
\end{array}\right]+\underbrace{\left[\begin{array}{ll}
2 & 0 \\
0 & 2 \\
0 & 0 \\
0 & 0
\end{array}\right]}_{\boldsymbol{B}}\left[\begin{array}{l}
v_{1} \\
v_{2}
\end{array}\right]}
\end{aligned}
$$

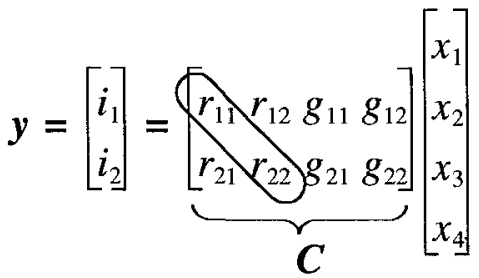

(6.20)

Assuming that the above poles are identified by (6.18), the residues shown encircled in (6.20) are perturbed in the proposed algorithm. Consequently, $\Delta \boldsymbol{C}$ for this example can be represented as:

$$
\Delta C=\left[\begin{array}{cccc}
\Delta r_{11} & 0 & 0 & 0 \\
0 & \Delta r_{22} & 0 & 0
\end{array}\right]
$$

and the corresponding unknown vector $Q$ in (6.17) is $Q=\left[\Delta r_{11} \Delta r_{22}\right]^{T}$. For the general case of $m$-port network with $n$ pair of complex poles, if the $l^{\text {th }}$ pole pair is selected by $(6.18)$, then the corresponding $\Delta C$ has the following form,

$$
\begin{aligned}
& \text { column } \\
& 2 m(l-1)+1 \\
& \downarrow
\end{aligned}
$$

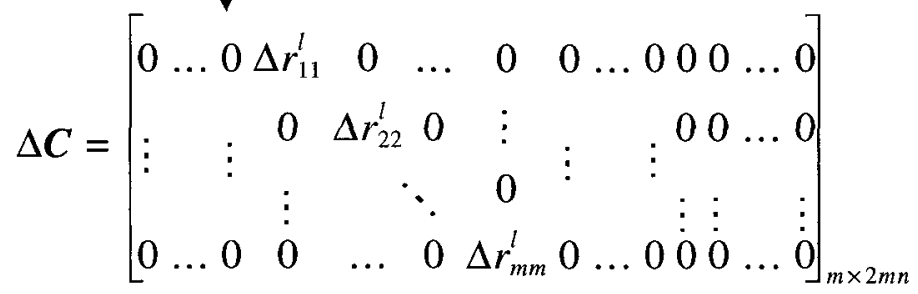




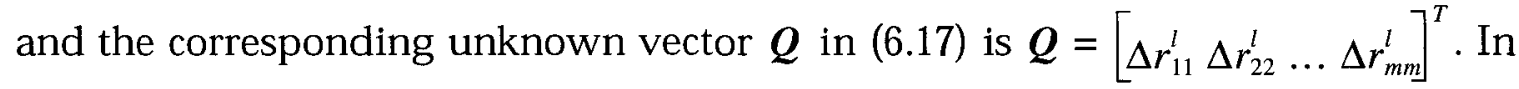
case more poles are involved, then $\Delta C$ will have block diagonal entries for the corresponding poles similar to the one described by (6.22) and vector $\boldsymbol{Q}$ is formulated accordingly.

With the residues for perturbation selected as per above guidelines, (6.17) is solved to offset $-\Delta \lambda$ (obtained as per the guidelines in Section 6.3.1), the eigenvalue of $\boldsymbol{F}(j \omega)$ at the frequency point of maximum violation. This process is continued at the point of maximum passivity violation in each passivity violation regions.

The advantage of the proposed perturbation (of the diagonal elements of $\boldsymbol{F}(j \omega)$ ) is that, it always adds positively to the eigenvalues of $\boldsymbol{F}(j \omega)$ and hence does not lead to a new passivity violation at any other frequency point. This can be proved using the following two Lemmas:

Lemma 1: The real part of the frequency response of a pole is linearly proportional to the real part of its residue.

Proof of Lemma 1: For the purpose of illustration, consider the response $H_{c}(s)$ of a complex pole pair, $-p^{\prime} \pm j p^{\prime \prime}$, with the corresponding residue $r^{\prime} \pm j r^{\prime \prime}$ :

$$
H_{c}(s)=\frac{r^{\prime}+j r^{\prime \prime}}{s+p^{\prime}-j p^{\prime \prime}}+\frac{r^{\prime}-j r^{\prime \prime}}{s+p^{\prime}+j p^{\prime \prime}}
$$

Separating (6.23) into real $(R(s))$ and imaginary $(I(s))$ parts we get 


$$
\begin{gathered}
H_{c}(s)=\underbrace{\frac{2 r^{\prime} p^{\prime}\left(p^{\prime 2}+p^{\prime \prime 2}+\omega^{2}\right)-2 r^{\prime \prime} p^{\prime \prime}\left(p^{\prime 2}+p^{\prime \prime 2}-\omega^{2}\right)}{\left(p^{\prime 2}+p^{\prime \prime 2}-\omega^{2}\right)^{2}+4 p^{\prime 2} \omega^{2}}+}_{\text {Real Part }(R(s))} \\
\underbrace{\frac{\left(r^{\prime} p^{\prime}-r^{\prime \prime} p^{\prime \prime}\right)\left(-4 p^{\prime} \omega\right)+2 r^{\prime} \omega\left(p^{\prime 2}+p^{\prime \prime 2}-\omega^{2}\right)}{\left(p^{\prime 2}+p^{\prime 2}-\omega^{2}\right)^{2}+4 p^{\prime 2} \omega^{2}}}_{\text {Imaginary Part }(I(s))}
\end{gathered}
$$

From (6.24) it is evident that the real part of the response of a complex pole is linearly proportional to the real part of its residue. Similarly, it can be easily shown that the above is true for the case of real poles too.

Lemma 2: If the real part of an $m$-port symmetric admittance matrix (represented by $\boldsymbol{F}(j \omega))$ is perturbed by a diagonal matrix $\Delta \boldsymbol{F}$, where

$$
\Delta \boldsymbol{F}=\left[\begin{array}{ccc}
\Delta F_{11}(\omega) & & \\
& \Delta F_{22}(\omega) & \\
& \ddots & \\
& & \Delta F_{m m}(\omega)
\end{array}\right] ; \quad \Delta F_{11}(\omega), \ldots \Delta F_{m m}(\omega)>0 ; \quad(\omega \in \Re \cup \infty)
$$

then the contribution of this perturbation to the eigenvalues of $\boldsymbol{F}(j \omega)$ is nonnegative throughout the frequency spectrum.

Proof of Lemma 2: Let $\boldsymbol{y}$ and $\boldsymbol{x}$ be the left and right eigenvectors of $\boldsymbol{F}(j \omega)$. Using the eigenvalue perturbation formulae [82], we can write the contribution of (6.25) to an eigenvalue of $\boldsymbol{F}(j \omega)$ as

$$
\Delta \lambda=\frac{\boldsymbol{y}^{T} \Delta \boldsymbol{F} \boldsymbol{x}}{\boldsymbol{y}^{T} \boldsymbol{x}} ; \quad \boldsymbol{x}=\left[x_{1}, \ldots x_{m}\right]^{T} ; \quad \boldsymbol{y}^{T}=\left[y_{1}, \ldots y_{m}\right]
$$


Noting that $\boldsymbol{F}(j \omega)$ is a real symmetric matrix and the fact that the left eigenvector $\boldsymbol{y}$ is equal to the right eigenvector $\boldsymbol{x}$ for a real symmetric matrix, we can write (6.26) as

$$
\Delta \lambda=\frac{\Delta F_{11}(\omega) x_{1}^{2}+\Delta F_{22}(\omega) x_{2}^{2}+\ldots+\Delta F_{m m}(\omega) x_{m}{ }^{2}}{x_{1}{ }^{2}+x_{2}{ }^{2}+\ldots+x_{m}{ }^{2}}
$$

It is evident from (6.27) that the contribution of the proposed perturbation (of the diagonal elements of $\boldsymbol{F}(j \omega))$ always adds positively to the eigenvalues of $\boldsymbol{F}(j \omega)$ and hence does not lead to the passivity violation at any other frequency point.

\subsection{2b Error Estimation}

As a result of perturbation of the residue matrix $C$ for compensation, there will be some error introduced in the time and frequency domain responses. An estimation of this error can be obtained in the similar way as described in Section 4.2 .4 a, by expressing the $L_{2}$ norm [81] of $\Delta \boldsymbol{Y}(j \omega)$ as

$$
\|\Delta \boldsymbol{Y}\|_{2}^{2}=\int_{-\infty}^{\infty}\|\Delta \boldsymbol{Y}(j \omega)\|_{F}^{2} d \omega=\int_{-\infty i=1 j=1}^{\infty} \sum_{i j}^{m}\left|\Delta Y_{i j}(j \omega)\right|^{2} d \omega=\int_{-\infty i=1}^{\infty} \sum_{j=1}^{m} \sum_{i j}^{m}\left|\Delta Y_{i j}(t)\right|^{2} d t=\operatorname{trace}\left(\Delta \boldsymbol{C} \boldsymbol{P} \Delta \boldsymbol{C}^{T}\right)
$$

where $\|\Delta \boldsymbol{Y}(j \omega)\|_{F}$ is the Frobenius norm of $\Delta \boldsymbol{Y}(j \omega), m$ is the number of ports and $\boldsymbol{P}$ is the controllability grammian obtained by solving the following Lyapunov equation

$$
\boldsymbol{A P}+\boldsymbol{P} \boldsymbol{A}^{T}+\boldsymbol{B} \boldsymbol{B}^{T}=\mathbf{0}
$$

With matrices $\boldsymbol{A}, \boldsymbol{B}, \boldsymbol{P}$ being constant, it is evident from (6.28) that keeping $\|\Delta C\| \ll\|C\|$ will keep the error in the time and frequency domain to the minimum. 
A summary of the steps involved in the proposed passivity enforcement algorithm is given in Fig. 6.2, in the form of pseudocode.

- Step 1: Obtain multiport tabulated data up to $f_{\max }$ (maximum frequency of interest).

- Step 2: Compute the multiport pole-residue model (6.1). Obtain the state-space system $(\boldsymbol{A}, \boldsymbol{B}, \boldsymbol{C}, \boldsymbol{D})(6.2)$.

- Step 3: Construct the Hamiltonian matrix and check its eigenvalue (6.6).

if no imaginary eigenvalues are found, macromodel is passive. Go to End

else a) Determine the singular locations of $\boldsymbol{F}(j \omega)$ using Theorem 2 and collect them in a vector $S_{a}=\left[\omega_{1}, \omega_{2} \ldots \omega_{T}\right]$ such that $\omega_{1}<\omega_{2}<\ldots<\omega_{T}$, where ' $T$ ' is the total number of such entries. Let $\omega_{H}=\omega_{T}$.

b) Determine the regions of violation using the following steps as outlined in Section 6.3.1:

(i) At the frequency corresponding to each of the entries in the vector $\boldsymbol{S}_{a}$, evaluate the slope of the eigenvalue of $\boldsymbol{F}(j \omega)$ using (6.13).

(ii) Count the number of positive and negative slopes starting from $\omega_{H}$. When the count of positive and negative slopes become equal, say at $\omega_{k}$, then the first region of local passivity violation is established (i.e. the region between $\omega_{H}$ to $\omega_{k}$ ).

(iii) Reset the count of slope to zero and designate $\omega_{H}=\omega_{k-1}$ and repeat steps ( $i$ ) and (iii) until all entries in the set $S_{a}$ are exhausted.

c) Determine the exact location of maximum passivity violation in every region of violation using (6.14).

d) Identify the poles used for compensation using (6.18) and then select the corresponding residues of driving point admittances (as per the guidelines in Section 6.3.2) and formulate $\Delta \boldsymbol{C}$.

- Step 4: Perform the passivity compensation by computing $\Delta \boldsymbol{C}$ at each point of maximum violation determined in c) using (6.17). Go to step 3.

End

Fig. 6.2. Pseudocode for the proposed passivity compensation algorithm 


\section{$6.4 \quad$ Numerical Results}

In this section, two examples are presented to demonstrate the efficiency and accuracy of the proposed compensation algorithm. The tabulated $Y$-parameter data for these examples were obtained from networks consisting of several interconnects and lumped circuit elements.

\subsubsection{Example 1: Three Port Interconnect Network}

In this example, the proposed compensation algorithm was performed on the tabulated data obtained from a 3-port interconnect subnetwork [24] (Fig. 6.3). The subnetwork was characterized by a set of tabulated data ( $Y$-parameters) up to $6 \mathrm{GHz}$ (henceforth referred to as the original data). The data was fitted using the algorithm described in [26] with 40 complex and 4 real poles, as listed in Table 6.1. The corresponding state-space macromodel was tested for passivity using Theorem 1 of Section 6.2 , by computing the eigenvalues of the Hamiltonian matrix (6.6). For this macromodel, six imaginary eigenvalues were found, indicating that the macromodel was non-passive. The details of the eigenvalue spectrum of the Hamiltonian matrix are shown in Fig. 6.4(a). For the purpose of clarity, an enlarged view of the eigenvalue spread near the imaginary axis showing the exact numerical values of the imaginary eigenvalues is presented in Fig. 6.4(b). According to Theorem-2 of Section 6.2, these imaginary eigenvalues correspond to the exact locations at which eigenvalues of $\operatorname{Real}(\boldsymbol{Y}(j \omega))$ become zero. Fig. 6.5 confirms this result by showing the eigenvalue spectrum of $\operatorname{Real}(\boldsymbol{Y}(j \omega))$. As seen in this figure, $\operatorname{Real}(\boldsymbol{Y}(j \omega))$ becomes singular at six frequency points, which correspond exactly to the imaginary eigenvalues of the Hamiltonian matrix. 
The regions of passivity violation were then determined using the method in Section 6.3.1. In this example three regions of passivity violation were found and they are indicated in both Fig. 6.5 and Fig. 6.6. In each region, the location of maximum passivity violation were determined using (6.14) of Section 6.3.1, and the corresponding details are given in Table 6.2

With the above information of exact locations of passivity violation, passivity correction was performed using the steps proposed in Section 6.3.2, by perturbing the selected residues of diagonal elements of the admittance matrix. The details of poles and selected residues (before and after perturbation) and the relative norm of the perturbed residue matrix $(\|\Delta C\| /\|C\|)$ are summarized in the Table 6.2

Fig. 6.6 shows the eigenvalue spectrum of $\operatorname{Real}(\boldsymbol{Y}(j \omega))$ before and after compensation. As indicated by the dotted line, all violations were corrected by the proposed algorithm. This is also verified by formulating the Hamiltonian matrix (6.6) of the compensated macromodel. Fig. 6.7 and Fig. 6.8 show the comparison between the original data and the response of the proposed passive macromodel and they match accurately. The MATLAB execution of the proposed algorithm on a Sun-Blade-100 machine required 6.49 seconds for this example.

Next, the passive macromodel was linked to HSPICE and a non-linear transient analysis performed, for an input pulse having a rise and fall time of $0.1 \mathrm{~ns}$ and pulse width of 5 ns. The results at node $\mathbf{P}_{1}$ and $\boldsymbol{V}_{\text {out } 2}$ are shown in Fig. 6.9. For validation purposes, the original network (from which the tabulated data was obtained), was also subjected to the transient analysis (using HSPICE) with similar input and terminations, and the results are compared in Fig. 6.9. As seen, both match accurately. 
It is to be noted that, while using the proposed global passivity enforcement algorithm, no additional regions of passivity violation were introduced during the compensation process (since it employs a guaranteed search direction for enforcing passivity). On the other hand, for comparison purposes, when the passivity compensation algorithms of [40], [41] were used, two new passivity violation regions were introduced while performing the compensation at the above three regions. It is to be noted that, this problem could be aggravated in the presence of many regions of passivity violations in the original macromodel and also for macromodels with large number of ports.

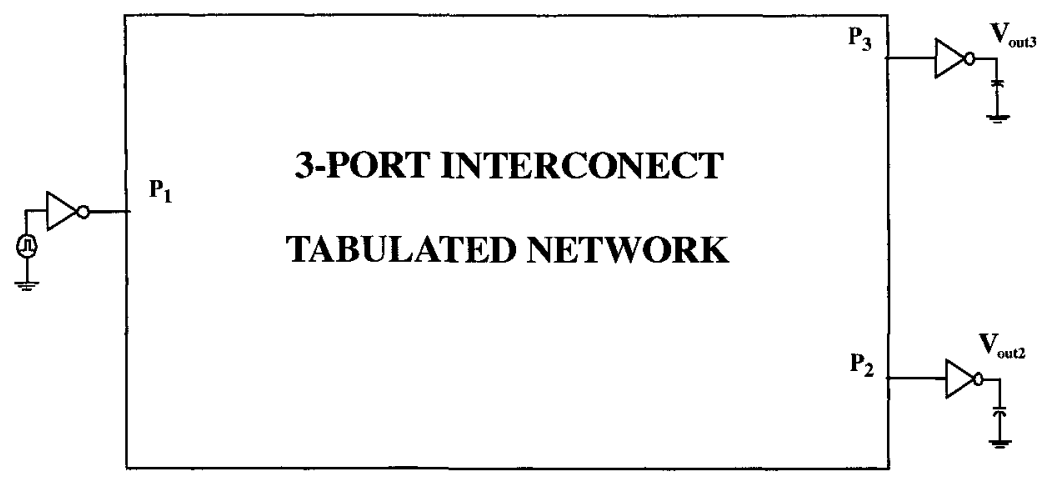

Fig. 6.3. 3-port interconnect network 
Table. 6.1. List of poles for example 1

\begin{tabular}{|c|}
\hline Poles for Example 1 \\
\hline$-9.3844 \mathrm{e}-002 \pm 3.8276 \mathrm{e}+001 \mathrm{i}$ \\
\hline$-1.5689 \mathrm{e}-001 \pm 3.6162 \mathrm{e}+001 \mathrm{i}$ \\
\hline$-2.9388 \mathrm{e}-001 \pm 3.4761 \mathrm{e}+001 \mathrm{i}$ \\
\hline$-6.8818 \mathrm{e}-002 \pm 3.2396 \mathrm{e}+001 \mathrm{i}$ \\
\hline$-9.5903 \mathrm{e}-002 \pm 3.1124 \mathrm{e}+001 \mathrm{i}$ \\
\hline$-1.8371 \mathrm{e}-001 \pm 2.8294 \mathrm{e}+001 \mathrm{i}$ \\
\hline$-1.6891 \mathrm{e}-001 \pm 2.8017 \mathrm{e}+001 \mathrm{i}$ \\
\hline$-1.5723 \mathrm{e}-001 \pm 2.4263 \mathrm{e}+001 \mathrm{i}$ \\
\hline$-2.9318 \mathrm{e}-001 \pm 2.3702 \mathrm{e}+001 \mathrm{i}$ \\
\hline$-1.2720 \mathrm{e}-001 \pm 2.1614 \mathrm{e}+001 \mathrm{i}$ \\
\hline$-3.1590 \mathrm{e}-001 \pm 1.9997 \mathrm{e}+001 \mathrm{i}$ \\
\hline$-6.3208 \mathrm{e}-001 \pm 1.8482 \mathrm{e}+001 \mathrm{i}$ \\
\hline$-2.2797 \mathrm{e}-001 \pm 1.5844 \mathrm{e}+001 \mathrm{i}$ \\
\hline$-2.6499 \mathrm{e}-001 \pm 1.3414 \mathrm{e}+001 \mathrm{i}$ \\
\hline$-3.7967 \mathrm{e}-001 \pm 1.2015 \mathrm{e}+001 \mathrm{i}$ \\
\hline$-3.2720 \mathrm{e}-001 \pm 9.1904 \mathrm{e}+000 \mathrm{i}$ \\
\hline$-3.6646 \mathrm{e}-001 \pm 7.7105 \mathrm{e}+000 \mathrm{i}$ \\
\hline$-1.1781 \mathrm{e}+000 \pm 6.7644 \mathrm{e}+000 \mathrm{i}$ \\
\hline$-8.8563 \mathrm{e}-001 \pm 5.3921 \mathrm{e}+000 \mathrm{i}$ \\
\hline$-1.3786 \mathrm{e}+000 \pm 3.5099 \mathrm{e}+000 \mathrm{i}$ \\
\hline
\end{tabular}


Table. 6.2. Details of proposed passivity correction algorithm for example 1

\begin{tabular}{|c|c|c|c|}
\hline & $\begin{array}{c}\text { Region of Violation } \\
\text { No.1 }\end{array}$ & $\begin{array}{c}\text { Region of Violation } \\
\text { No. } 2\end{array}$ & $\begin{array}{c}\text { Region of Violation } \\
\text { No.3 }\end{array}$ \\
\hline$f_{l}$ & $4.93 \mathrm{GHz}$ & $2.87 \mathrm{GHz}$ & $2.14 \mathrm{GHz}$ \\
\hline$f_{h}$ & $4.95 \mathrm{GHz}$ & $2.96 \mathrm{GHz}$ & $2.19 \mathrm{GHz}$ \\
\hline $\begin{array}{l}\text { Location of Maxi- } \\
\text { mum Violation }\end{array}$ & $4.9449 \mathrm{GHz}$ & $2.9230 \mathrm{GHz}$ & $2.1605 \mathrm{GHz}$ \\
\hline $\begin{array}{l}\text { Maximum Violation } \\
(\text { Eigenvalue of } F(j \omega))\end{array}$ & $-4.5944 \mathrm{e}-4$ & $-3.4105 e-4$ & $-1.4078 \mathrm{e}-4$ \\
\hline $\begin{array}{l}\text { Poles Considered dur- } \\
\text { ing Compensation }\end{array}$ & $\begin{array}{l}-6.8818 \mathrm{e}-2 \pm 3.2396 \mathrm{e}+1 \mathrm{i} \\
-9.5903 \mathrm{e}-2 \pm 3.1124 \mathrm{e}+1 \mathrm{i} \\
-1.8371 \mathrm{e}-1 \pm 2.8294 \mathrm{e}+1 \mathrm{i}\end{array}$ & $\begin{array}{l}-3.1590 \mathrm{e}-1 \pm 1.9997 \mathrm{e}+1 \mathrm{i} \\
-6.3208 \mathrm{e}-1 \pm 1.8482 \mathrm{e}+1 \mathrm{i} \\
-2.2797 \mathrm{e}-1 \pm 1.5844 \mathrm{e}+1 \mathrm{i}\end{array}$ & $\begin{array}{l}-2.6499 \mathrm{e}-1 \pm 1.3414 \mathrm{e}+1 \mathrm{i} \\
-3.7967 \mathrm{e}-1 \pm 1.2015 \mathrm{e}+1 \mathrm{i}\end{array}$ \\
\hline \multicolumn{4}{|l|}{$\begin{array}{l}\text { Residues Prior to } \\
\text { Compensation }\end{array}$} \\
\hline$Y_{11}$ & $\begin{array}{l}4.8169 \mathrm{e}-4 \pm 7.6047 \mathrm{e}-6 \mathrm{i} \\
9.3618 \mathrm{e}-3 \pm 1.5375 \mathrm{e}-4 \mathrm{i} \\
1.3849 \mathrm{e}-2 \pm 1.1482 \mathrm{e}-3 \mathrm{i}\end{array}$ & $\begin{array}{l}3.6197 \mathrm{e}-3 \pm 2.8452 \mathrm{e}-4 \mathrm{i} \\
2.5574 \mathrm{e}-2 \pm 1.0757 \mathrm{e}-3 \mathrm{i} \\
4.3985 \mathrm{e}-3 \pm 9.5013 \mathrm{e}-4 \mathrm{i}\end{array}$ & $\begin{array}{l}2.5452 \mathrm{e}-3 \pm 2.2433 \mathrm{e}-3 \mathrm{i} \\
2.6578 \mathrm{e}-3 \pm 2.6693 \mathrm{e}-4 \mathrm{i}\end{array}$ \\
\hline$Y_{22}$ & $\begin{array}{l}2.8498 \mathrm{e}-4 \pm 3.5028 \mathrm{e}-6 \mathrm{i} \\
6.9539 \mathrm{e}-2 \pm 4.3490 \mathrm{e}-3 \mathrm{i} \\
2.9437 \mathrm{e}-2 \pm 1.6749 \mathrm{e}-3 \mathrm{i}\end{array}$ & $\begin{array}{l}9.8733 \mathrm{e}-4 \pm 3.2117 \mathrm{e}-4 \mathrm{i} \\
1.5195 \mathrm{e}-2 \pm 3.8187 \mathrm{e}-3 \mathrm{i} \\
3.6676 \mathrm{e}-4 \pm 6.9578 \mathrm{e}-4 \mathrm{i}\end{array}$ & $\begin{array}{l}7.2920 \mathrm{e}-2 \pm 7.3045 \mathrm{e}-3 \mathrm{i} \\
3.6715 \mathrm{e}-4 \pm 1.9476 \mathrm{e}-4 \mathrm{i}\end{array}$ \\
\hline$Y_{33}$ & $\begin{array}{l}4.3613 \mathrm{e}-2 \pm 4.1242 \mathrm{e}-3 \mathrm{i} \\
2.0500 \mathrm{e}-4 \pm 1.5760 \mathrm{e}-4 \mathrm{i} \\
1.8595 \mathrm{e}-2 \pm 3.8426 \mathrm{e}-4 \mathrm{i}\end{array}$ & $\begin{array}{l}5.1665 \mathrm{e}-2 \pm \quad 8.7504 \mathrm{e}-3 \mathrm{i} \\
-1.6837 \mathrm{e}-4 \pm 2.7814 \mathrm{e}-4 \mathrm{i} \\
4.6962 \mathrm{e}-2 \pm \quad 7.1124 \mathrm{e}-3 \mathrm{i}\end{array}$ & $\begin{array}{l}-6.3402 \mathrm{e}-5 \pm 4.6018 \mathrm{e}-4 \mathrm{i} \\
2.8735 \mathrm{e}-2 \pm 7.8639 \mathrm{e}-3 \mathrm{i}\end{array}$ \\
\hline \multicolumn{4}{|l|}{$\begin{array}{l}\text { Residues after Com- } \\
\text { pensation }\end{array}$} \\
\hline$Y_{11}$ & $\begin{array}{l}4.8169 \mathrm{e}-4 \pm 7.6047 \mathrm{e}-6 \mathrm{i} \\
9.3631 \mathrm{e}-3 \pm 1.5375 \mathrm{e}-4 \mathrm{i} \\
1.3849 \mathrm{e}-2 \pm 1.1482 \mathrm{e}-3 \mathrm{i}\end{array}$ & $\begin{array}{l}3.6343 \mathrm{e}-3 \pm 2.8452 \mathrm{e}-4 \mathrm{i} \\
2.5769 \mathrm{e}-2 \pm 1.0757 \mathrm{e}-3 \mathrm{i} \\
4.4031 \mathrm{e}-3 \pm 9.5013 \mathrm{e}-4 \mathrm{i}\end{array}$ & $\begin{array}{l}2.5506 \mathrm{e}-3 \pm 2.2433 \mathrm{e}-3 \mathrm{i} \\
2.6580 \mathrm{e}-3 \pm 2.6693 \mathrm{e}-4 \mathrm{i}\end{array}$ \\
\hline$Y_{22}$ & $\begin{array}{l}2.8498 \mathrm{e}-4 \pm 3.5028 \mathrm{e}-6 \mathrm{i} \\
6.9539 \mathrm{e}-2 \pm 4.3490 \mathrm{e}-3 \mathrm{i} \\
2.9437 \mathrm{e}-2 \pm 1.6749 \mathrm{e}-3 \mathrm{i}\end{array}$ & $\begin{array}{l}1.0082 \mathrm{e}-3 \pm 3.2117 \mathrm{e}-4 \mathrm{i} \\
1.5475 \mathrm{e}-2 \pm 3.8187 \mathrm{e}-3 \mathrm{i} \\
3.7328 \mathrm{e}-4 \pm 6.9578 \mathrm{e}-4 \mathrm{i}\end{array}$ & $\begin{array}{l}7.2920 \mathrm{e}-2 \pm 7.3045 \mathrm{e}-3 \mathrm{i} \\
3.6717 \mathrm{e}-4 \pm 1.9476 \mathrm{e}-4 \mathrm{i}\end{array}$ \\
\hline$Y_{33}$ & $\begin{array}{l}4.3614 \mathrm{e}-2 \pm 4.1242 \mathrm{e}-3 \mathrm{i} \\
2.7017 \mathrm{e}-4 \pm 1.5760 \mathrm{e}-4 \mathrm{i} \\
1.8595 \mathrm{e}-2 \pm 3.8426 \mathrm{e}-4 \mathrm{i}\end{array}$ & $\begin{array}{l}5.1666 \mathrm{e}-2 \pm 8.7504 \mathrm{e}-3 \mathrm{i} \\
-1.6623 \mathrm{e}-4 \pm 2.7814 \mathrm{e}-4 \mathrm{i} \\
4.6963 \mathrm{e}-2 \pm 7.1124 \mathrm{e}-3 \mathrm{i}\end{array}$ & $\begin{array}{l}-2.7867 \mathrm{e}-6 \pm 4.6018 \mathrm{e}-4 \mathrm{i} \\
2.8739 \mathrm{e}-2 \pm 7.8639 \mathrm{e}-3 \mathrm{i}\end{array}$ \\
\hline$\|\Delta C\| /\|C\|$ & $3.3888 \mathrm{e}-4$ & $1.4198 \mathrm{e}-3$ & $1.419801 \mathrm{e}-3$ \\
\hline
\end{tabular}




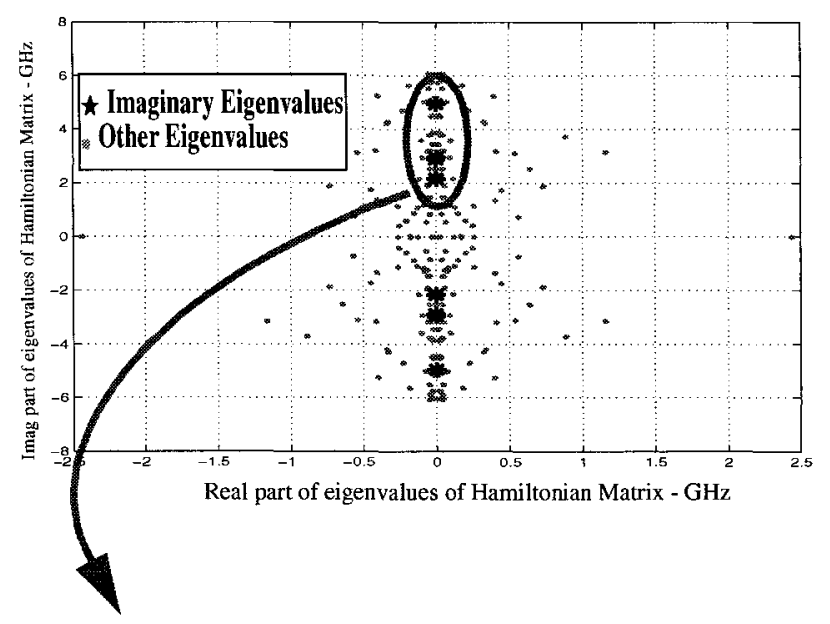

(a)

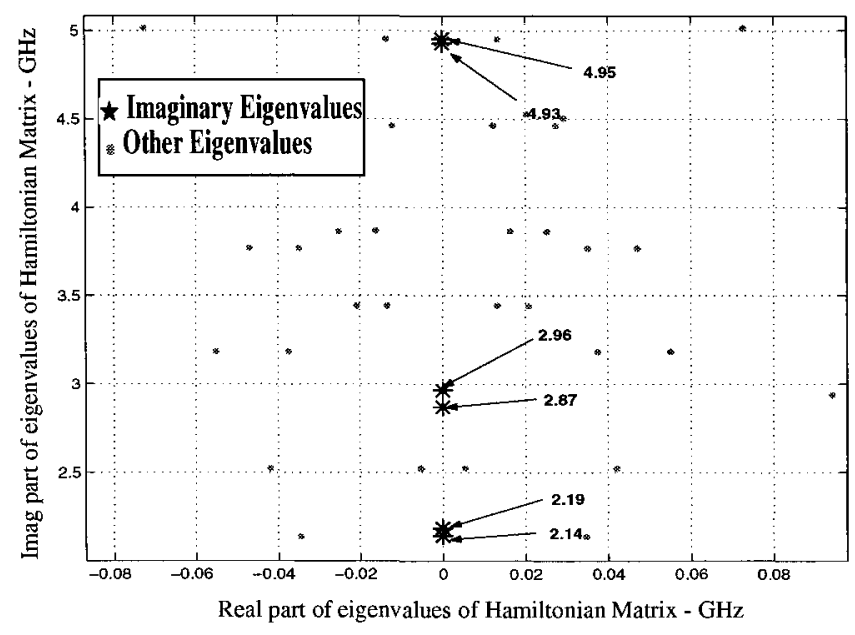

(b)

Fig. 6.4. (a) Eigenvalue spectrum of the Hamiltonian matrix. (b) Enlarged view of eigenvalue spectrum 


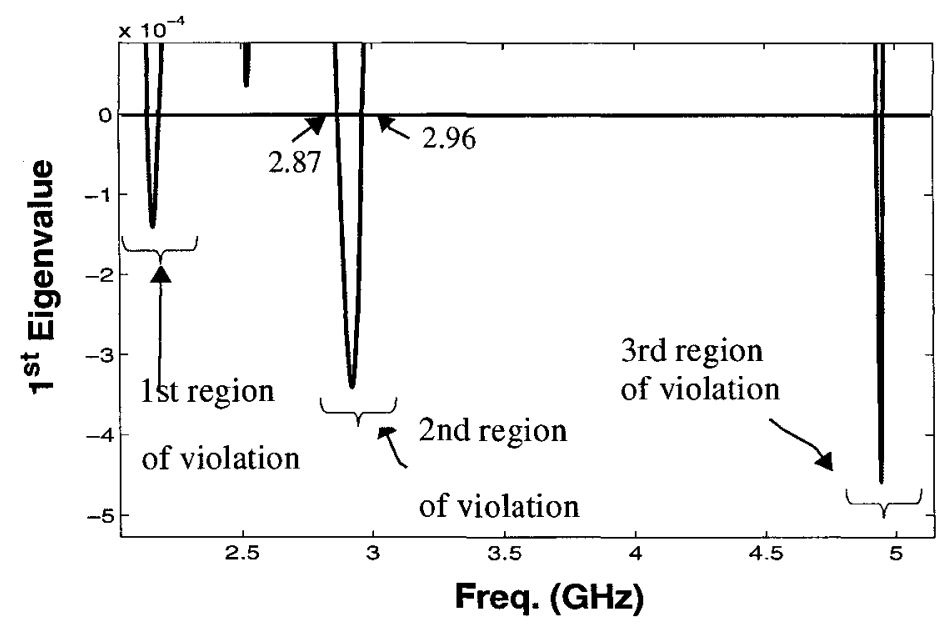

Fig. 6.5. Eigenvalue v/s frequency of $\operatorname{Real}(Y(j \omega))$ - example 1

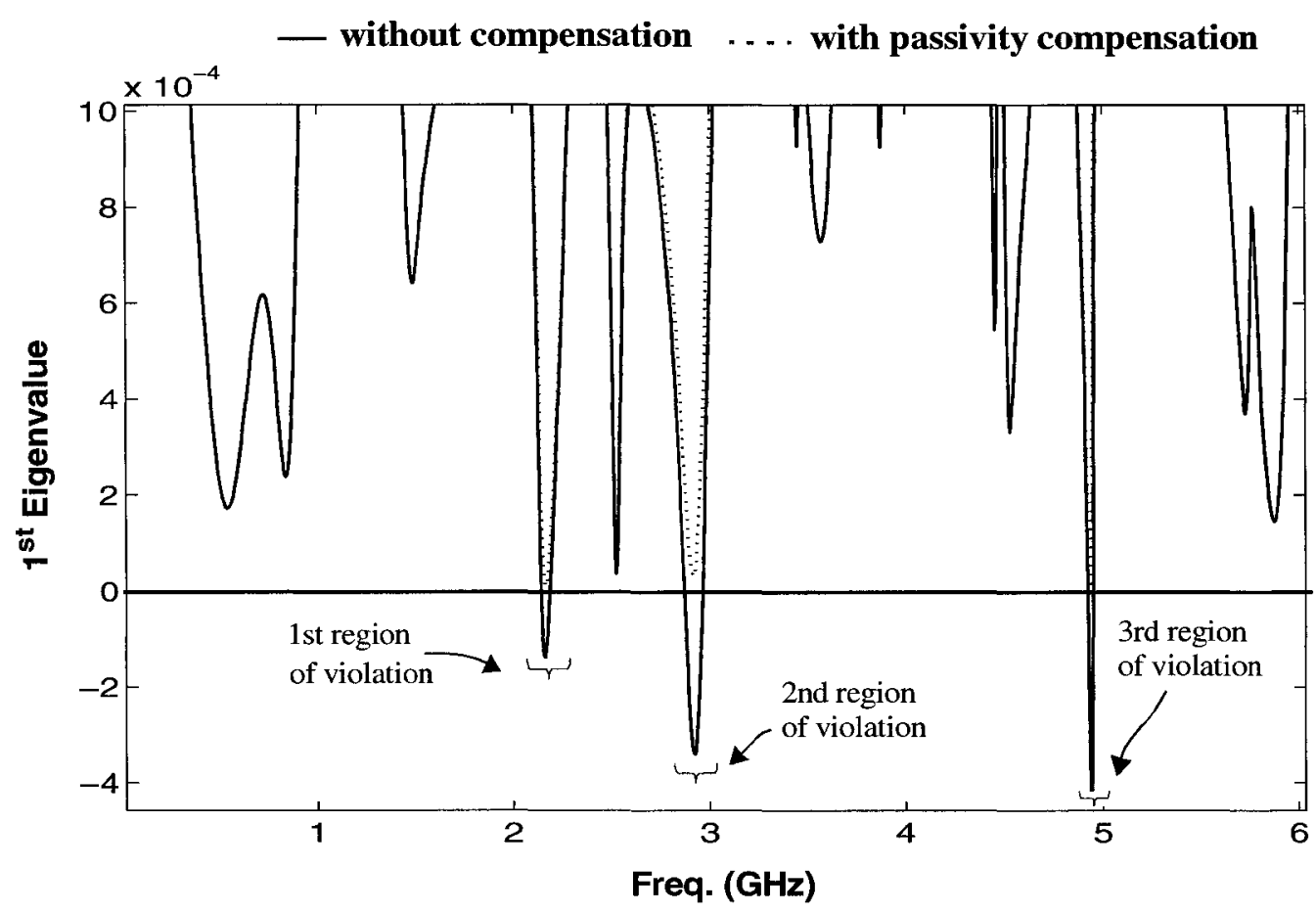

Fig. 6.6. Eigenvalue v/s frequency of $\operatorname{Real}(Y(j \omega))$ - with proposed passivity compensation 

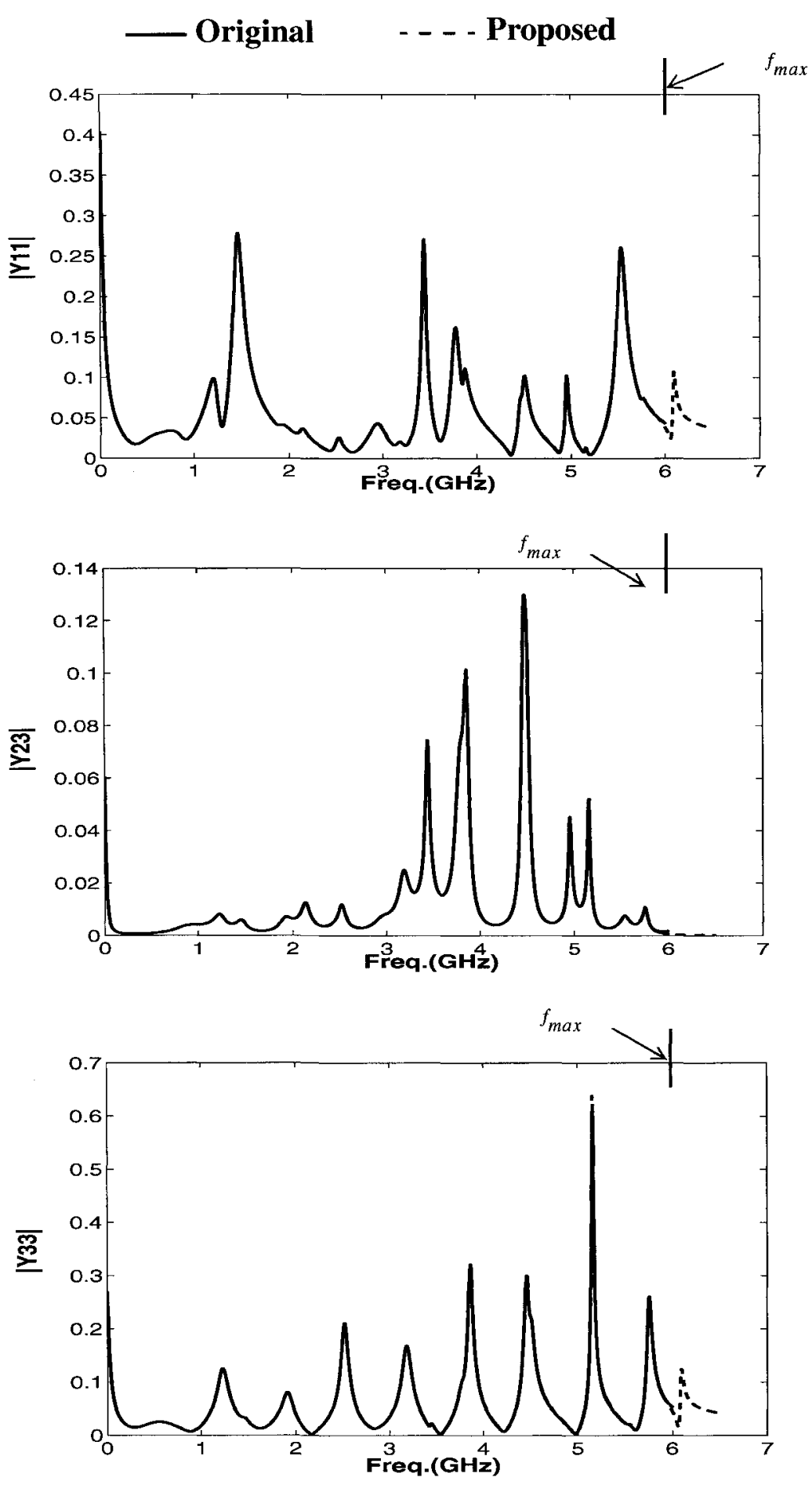

Fig. 6.7. Frequency responses (magnitude) - example 1 

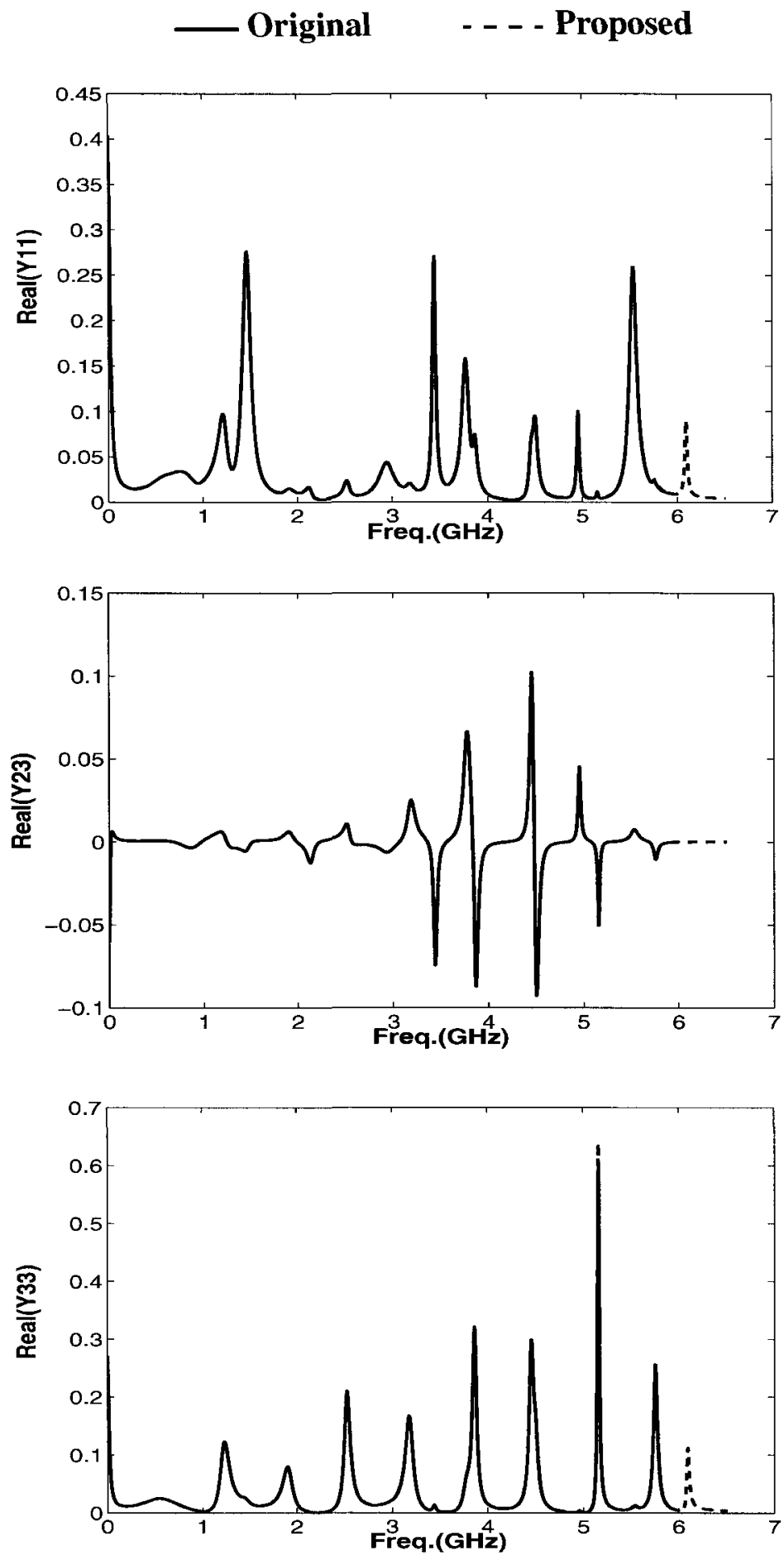

Fig. 6.8. Frequency responses (real part) - example 1 

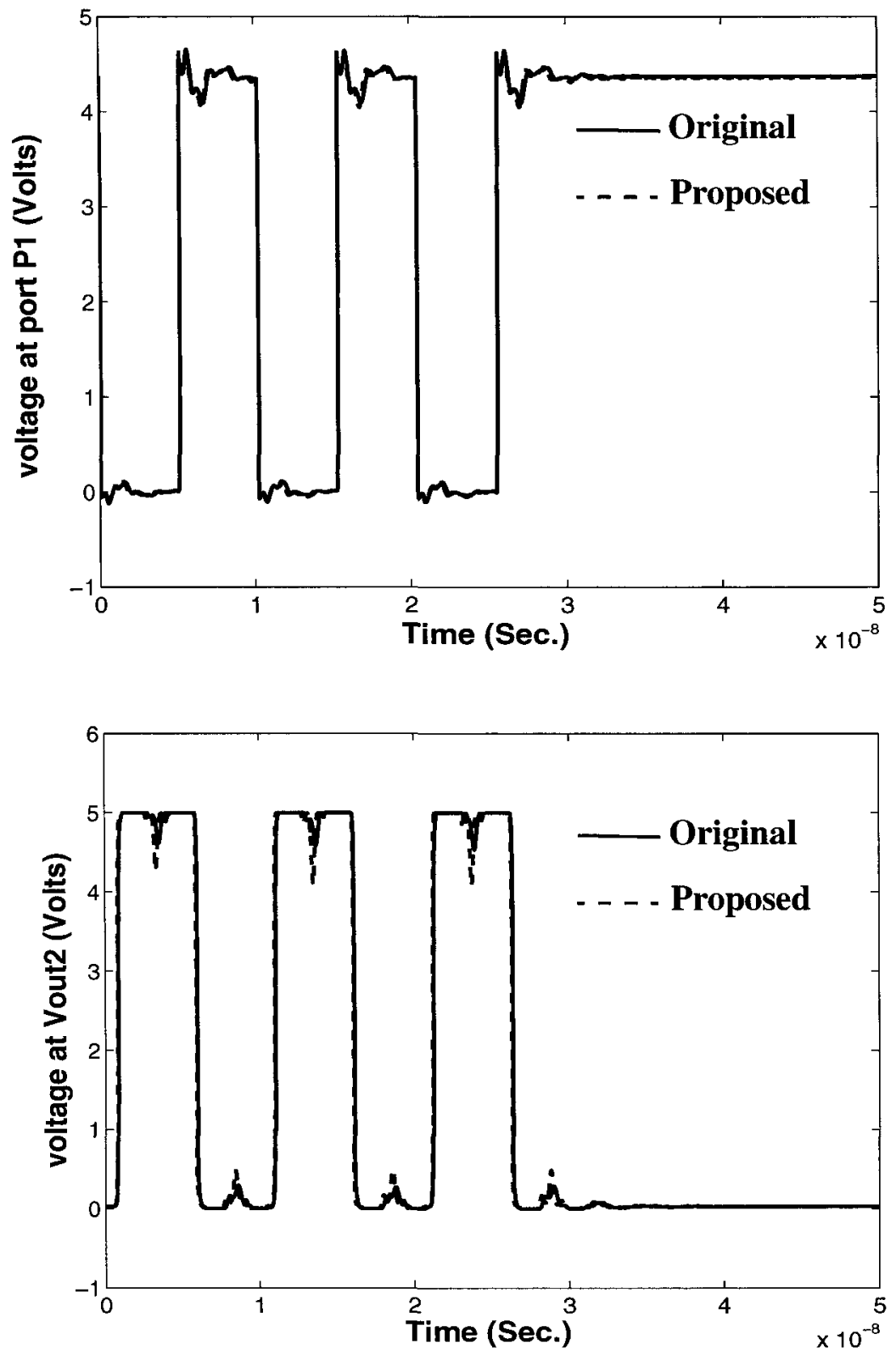

Fig. 6.9. Transient results of example 1 


\subsubsection{Example 2: Four Port Tabulated Data}

In this example, the proposed algorithm was performed on the tabulated $Y$ parameters of a four port interconnect subnetwork (Fig. 6.10). The data was fitted using the algorithm described in [26] (20 complex poles and 4 real poles were required, all were stable poles) and the state-space macromodel was obtained. The macromodel was tested for passivity using Theorem 1 of Section 6.2, and in this case two imaginary eigenvalues were found, indicating that the macromodel was non-passive. This is confirmed by the corresponding eigenvalue spectrum of $\operatorname{Real}(\boldsymbol{Y}(j \omega))$ which is shown in Fig. 6.11(a).

The regions of passivity violation were then determined using the method in Section 6.3.1. In this example, one region of passivity violation was found and is indicated in Fig. 6.11(a). In this region of violation, the location of maximum passivity violation was determined using (6.14) of Section 6.3.1, and the corresponding details are given in Table 6.3.

With the above information of exact location of passivity violation, passivity correction was performed using the algorithm proposed in Section 6.3.2, by perturbing the selected residues of diagonal elements of the admittance matrix. Due to the guaranteed search direction for enforcing passivity used in the algorithm, no additional regions of passivity violation were introduced during compensation. The details of the poles whose residues are perturbed and the relative norm of the perturbed residue matrix $\|\Delta C\| /\|C\|$ are summarized in the Table 6.3.

Fig. 6.11(b) shows the eigenvalue spectrum of $\operatorname{Real}(Y(j \omega))$ before and after the compensation. Fig. 6.12 and Fig. 6.13 show a sample of comparisons between the original data and the response of the proposed passive macromodel, and they 
match accurately. Next, a non-linear transient analysis is performed by replacing the four port linear network in Fig. 6.10 with the proposed macromodel, for an input pulse having a rise/fall time of 0.1 ns and pulse width of 5 ns (using HSPICE). For validation purposes, the original network from which the data was obtained, was also subjected to the transient analysis (using HSPICE) with the similar input and terminations, and the corresponding transient results are compared in Fig. 6.14. As seen, both match accurately.

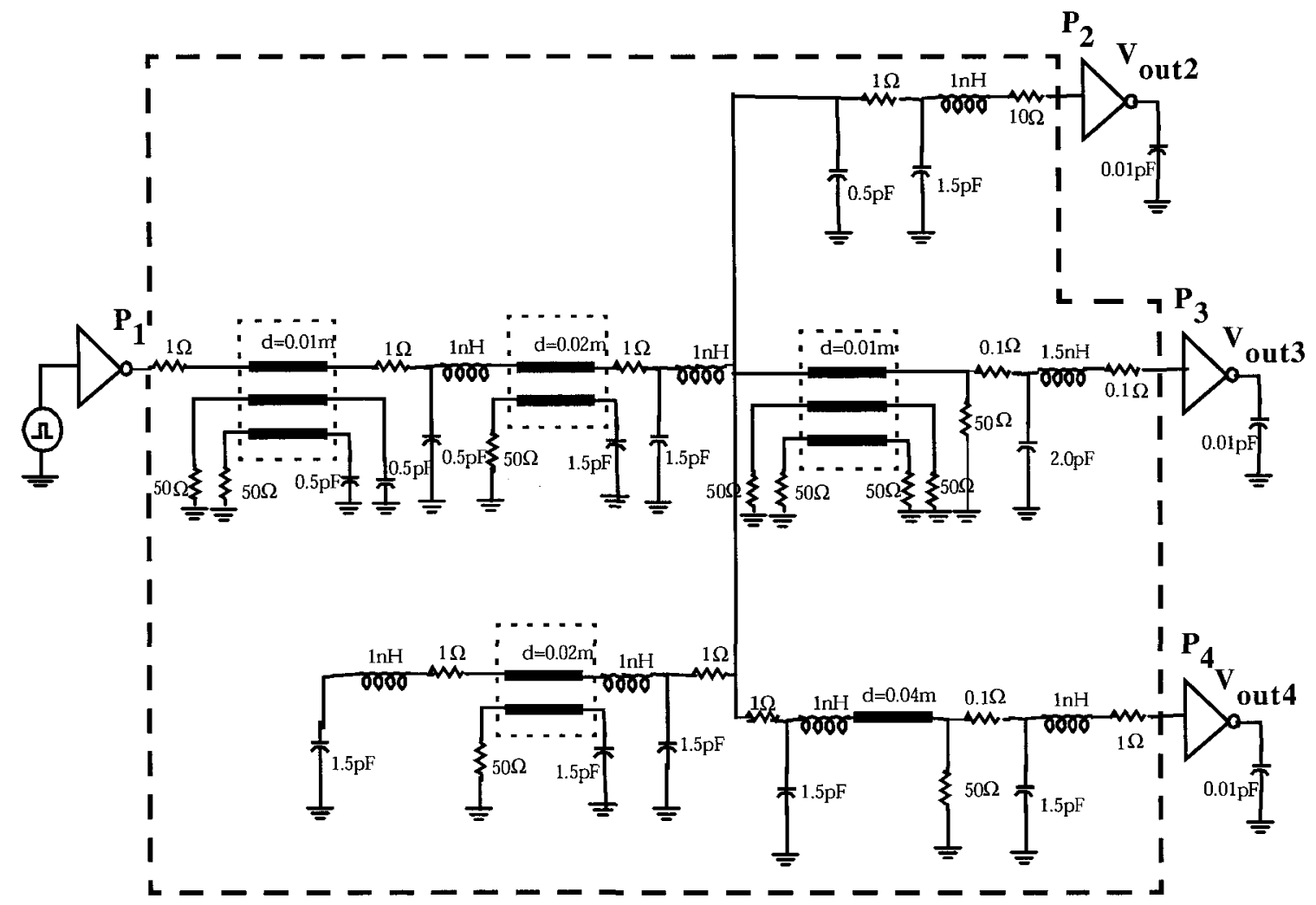

Fig. 6.10. Four port interconnect subnetwork - example 2 


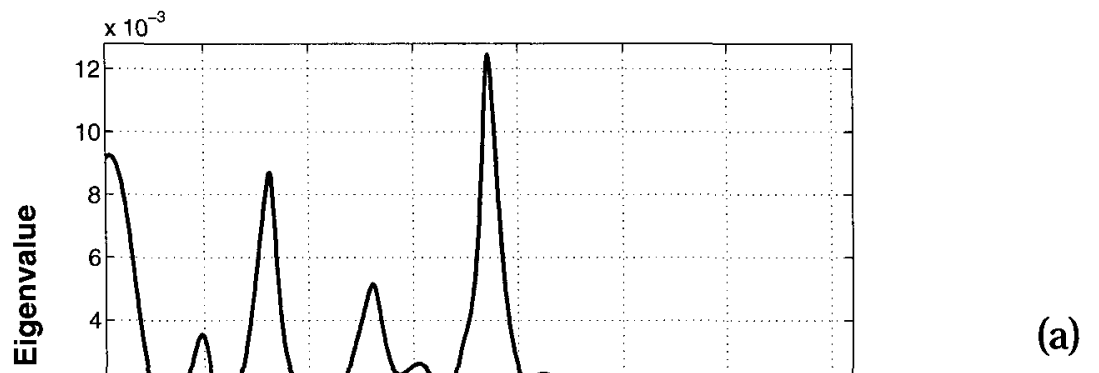

(b)

Fig. 6.11. Eigenvalues of Real $(Y(j \omega))$ - example 2. (a) Nonpassive macromodel (b) Enlarged view: Comparison of eigenvalues of nonpassive and compensated macromodels 
Table. 6.3. Details of proposed passivity correction algorithm for example 2

\begin{tabular}{|c|c|}
\hline & Region of Violation No.1 \\
\hline$f_{l}$ & $5.9327 \mathrm{GHz}$ \\
\hline$f_{h}$ & $6.2238 \mathrm{GHz}$ \\
\hline Location of Maximum Violation & $6.115 \mathrm{GHz}$ \\
\hline Maximum Violation(Eigenvalue of $\boldsymbol{F}(j \omega)$ ) & $-2.8923 \mathrm{e}-3$ \\
\hline Poles Considered during Compensation & $\begin{array}{l}-1.3150 \pm 4.0243 \mathrm{e}+1 \mathrm{i} \\
-2.4110 \mathrm{e}-1 \pm 3.4912 \mathrm{e}+1 \mathrm{i}\end{array}$ \\
\hline \multicolumn{2}{|l|}{ Residues Prior to Compensation } \\
\hline$Y_{11}$ & $\begin{array}{l}2.4078 \mathrm{e}-002 \pm 6.2584 \mathrm{e}-002 \mathrm{i} \\
8.3741 \mathrm{e}-002 \pm 9.1161 \mathrm{e}-004 \mathrm{i}\end{array}$ \\
\hline$Y_{22}$ & $\begin{array}{ll}1.1441 \mathrm{e}-003 \pm 1.7809 \mathrm{e}-003 \mathrm{i} \\
3.0684 \mathrm{e}-003 \pm 2.5928 \mathrm{e}-003 \mathrm{i}\end{array}$ \\
\hline $\boldsymbol{Y}_{33}$ & $\begin{array}{l}3.8586 \mathrm{e}-004 \pm 7.0019 \mathrm{e}-004 \mathrm{i} \\
2.4401 \mathrm{e}-004 \pm 1.7869 \mathrm{e}-004 \mathrm{i}\end{array}$ \\
\hline$Y_{44}$ & $\begin{array}{ll}2.5653 \mathrm{e}-003 \pm & 3.8721 \mathrm{e}-003 \mathrm{i} \\
3.3408 \mathrm{e}-004 \pm & 2.9410 \mathrm{e}-004 \mathrm{i}\end{array}$ \\
\hline \multicolumn{2}{|l|}{ Residues after Compensation } \\
\hline$Y_{11}$ & $\begin{array}{l}3.6665 \mathrm{e}-002 \pm 6.2584 \mathrm{e}-002 \mathrm{i} \\
8.4695 \mathrm{e}-002 \pm 9.1161 \mathrm{e}-004 \mathrm{i}\end{array}$ \\
\hline$Y_{22}$ & $\begin{array}{l}1.2194 \mathrm{e}-003 \pm 1.7809 \mathrm{e}-003 \mathrm{i} \\
3.0741 \mathrm{e}-003 \pm 2.5928 \mathrm{e}-003 \mathrm{i}\end{array}$ \\
\hline$Y_{33}$ & $\begin{array}{l}4.4661 \mathrm{e}-004 \pm 7.0019 \mathrm{e}-004 \mathrm{i} \\
2.4953 \mathrm{e}-004 \pm 1.7869 \mathrm{e}-004 \mathrm{i}\end{array}$ \\
\hline$Y_{44}$ & $\begin{array}{ll}2.5805 \mathrm{e}-003 \pm & 3.8721 \mathrm{e}-003 \mathrm{i} \\
3.3528 \mathrm{e}-004 \pm & 2.9410 \mathrm{e}-004 \mathrm{i}\end{array}$ \\
\hline$\|\Delta C\| /\|C\|$ & $1.39 \mathrm{e}-2$ \\
\hline
\end{tabular}



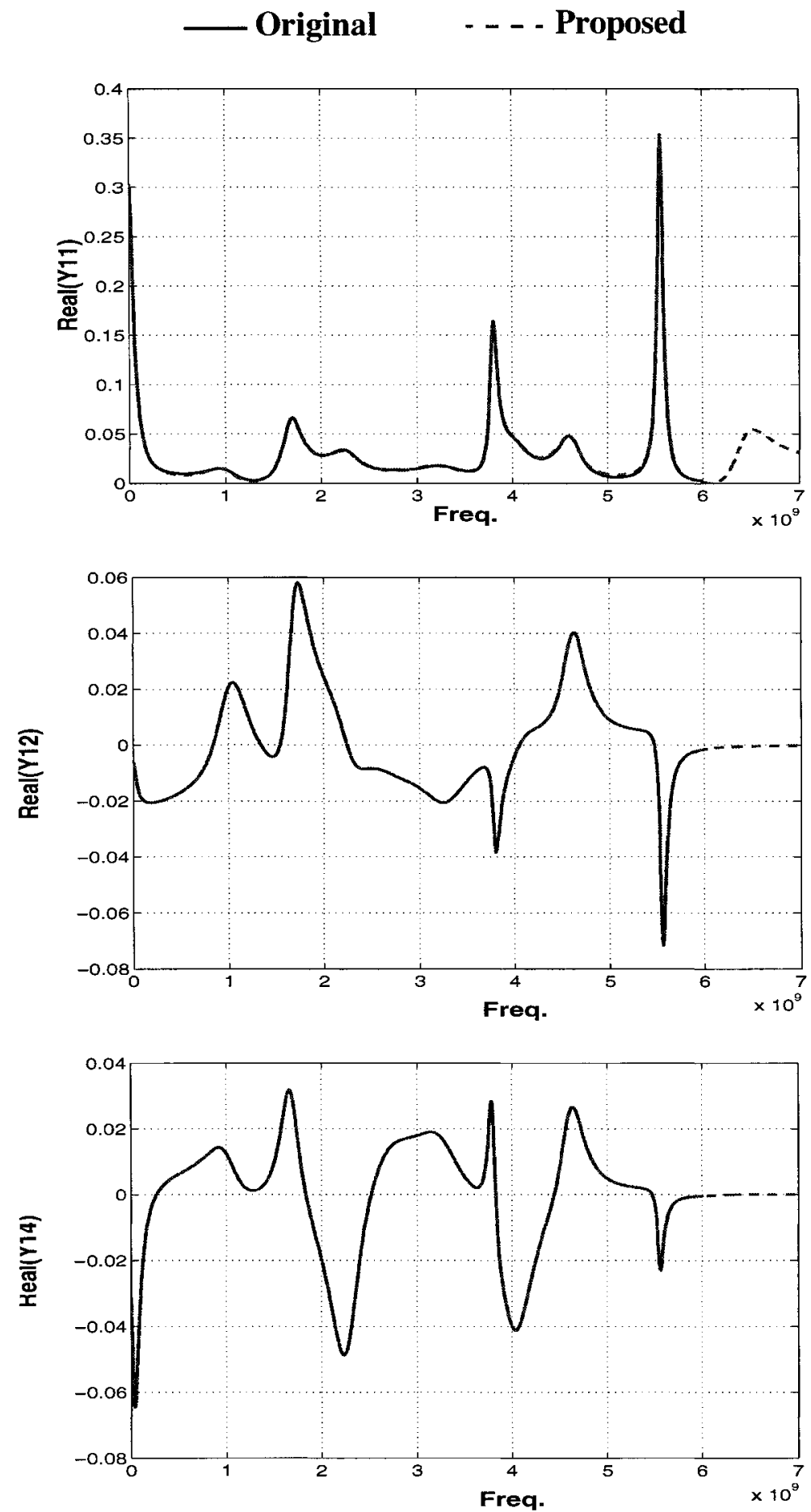

Fig. 6.12. Sample admittance parameters (real part) - example 2 

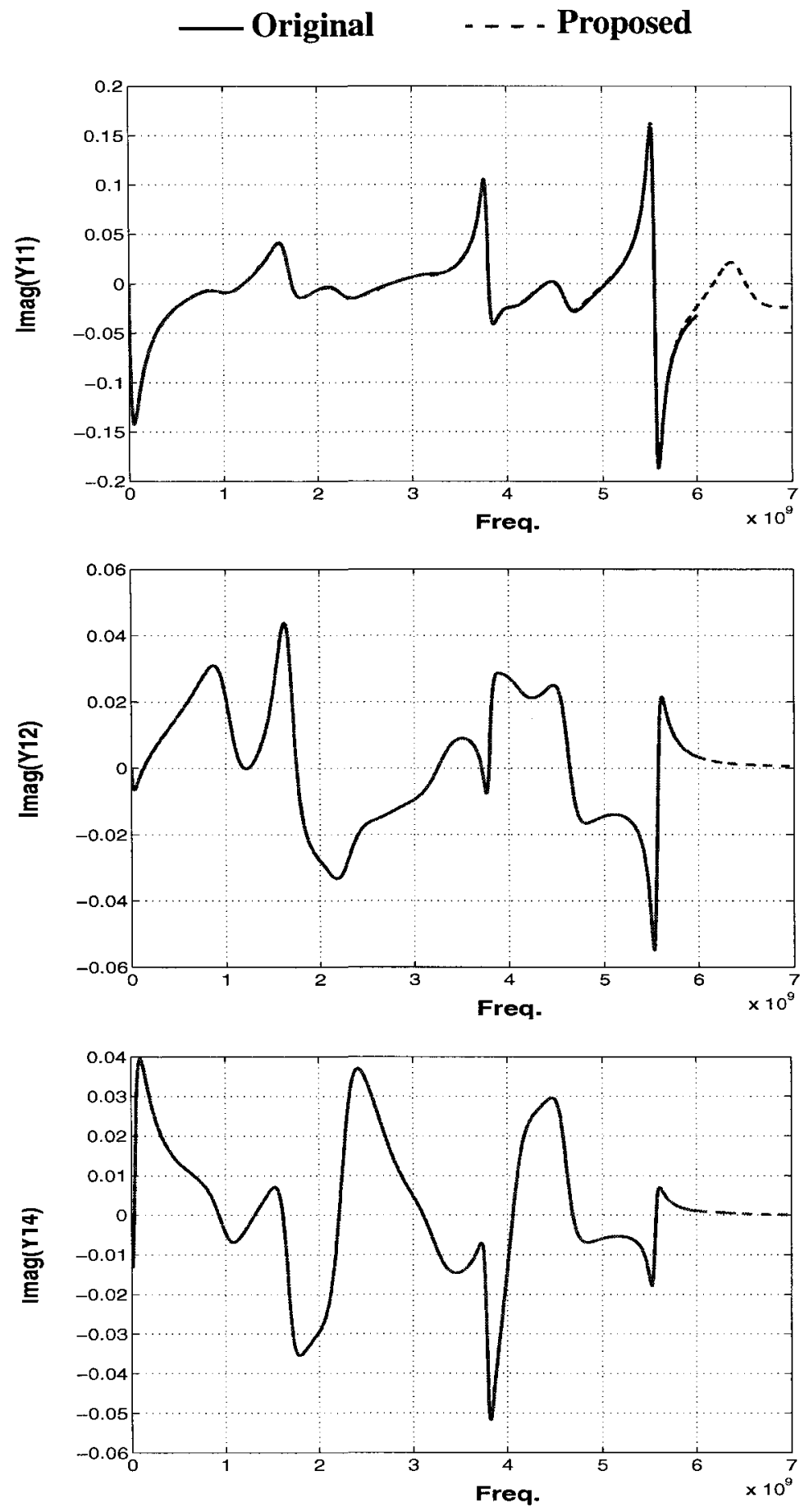

Fig. 6.13. Sample admittance parameters (imaginary part) - example 2 

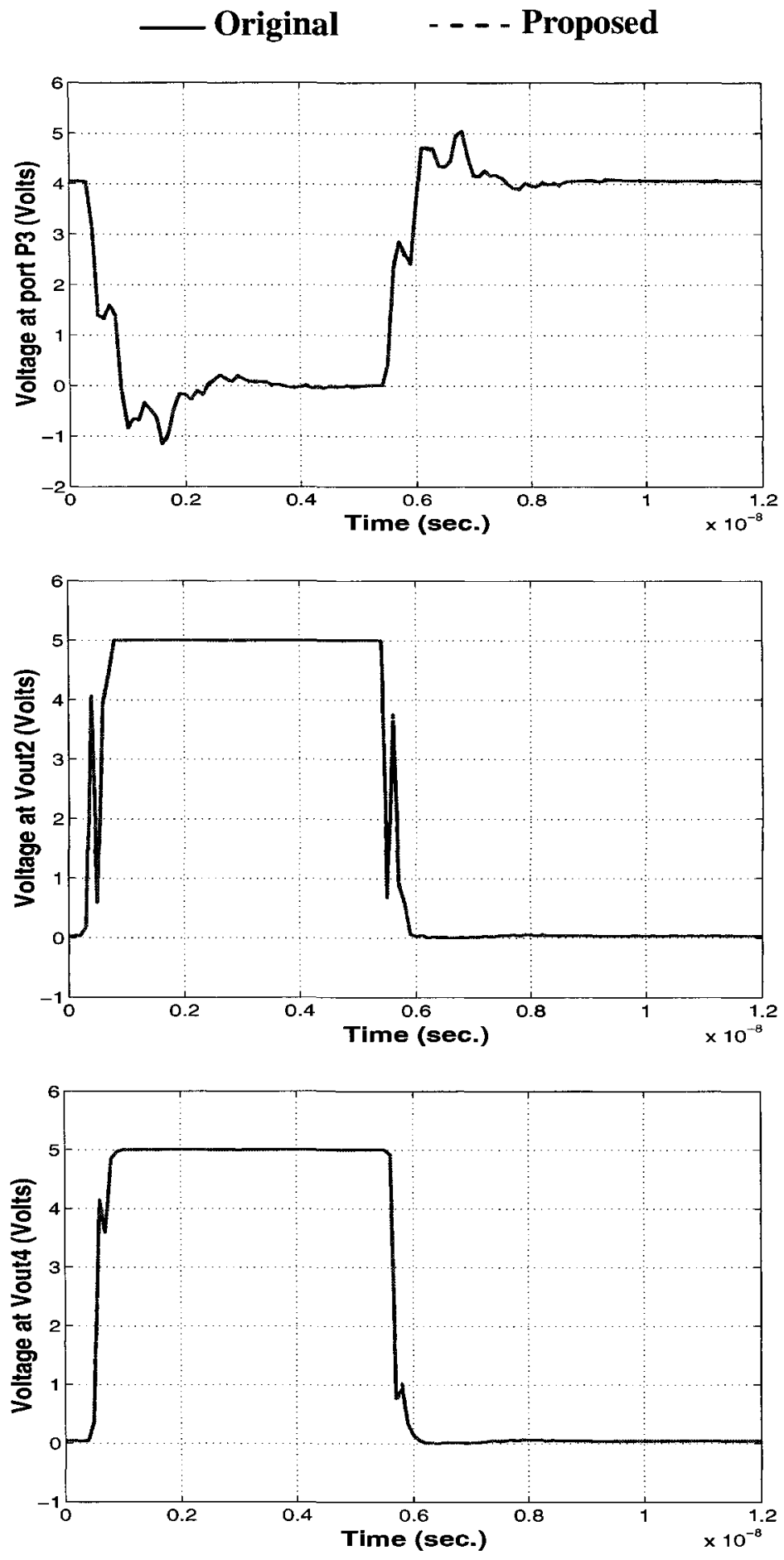

Fig. 6.14. Transient responses - example 2 


\subsection{Conclusion}

In this chapter, a passivity enforcement algorithm with a guaranteed search direction has been presented. The advantage of this algorithm is that, the passivity correction at one frequency point does not introduce any new passivity violation at other frequency points. As a result, the number of iterations and hence the total CPU time, required for passivity compensation is minimized. 


\section{CHAPTER 7}

\section{Global Passive Reduction Algorithm for RLC Interconnect Circuits with Embedded State- Space Systems}

In chapters 4, 5 and 6, we discussed the macromodeling of subnetworks described by tabulated data. With the ever increasing operating frequencies, complexities of modern high-speed designs, macromodeling based on tabulated data is gaining more and more importance. As a result, a typical signal path in a modern design could consist of diverse modules, such as, large lumped RLC network, distributed transmission lines (whose analytical models are available) and modules described by tabulated data (see Fig. 7.1). Currently, there is no model order reduction algorithm available, which can perform a unified reduction of the entire signal path consisting of diverse modules described above. The most suitable algorithm presently available for the model reduction of large systems is PRIMA. However, as pointed out in chapter 3, PRIMA is applicable to systems whose MNA matrices satisfy certain semi-positive definite criteria. The signal path comprising of diverse modules consists of some modules, such as distributed transmission lines, large lumped RLC networks, whose MNA matrices satisfy the criteria for PRIMA. However, modules described by tabulated data are generally approximated by passive state-space systems (as described in chapter 4, 5 and 6), whose matrices are not in the form suitable for PRIMA type of reduction. The most obvious approach to handle such a system consisting of diverse modules, is to analyze each of these modules separately using the most-appropriate algorithm available for that purpose (for example, measured subnetworks are characterized using 
algorithms such as [27]-[54], multiconductor transmission lines are macromodeled using algorithms such as [14], [15] etc.), and subsequently represent them by passive state-space equations. It is to be noted that, the size of each of these resulting state-space modules can be large (depending on the maximum frequency of interest and the complexity of data/structure) and can lead to slower simulation.

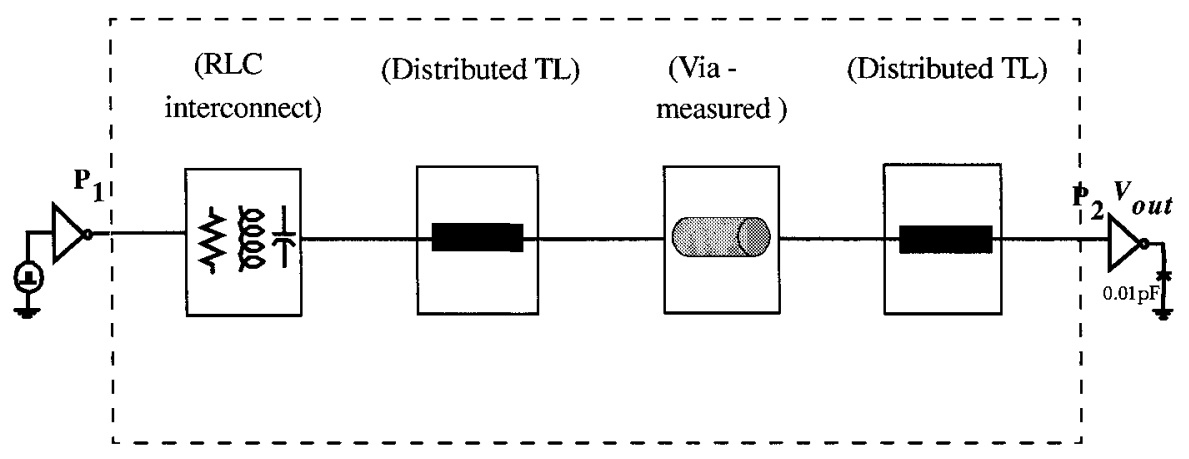

Fig. 7.1. A typical signal path consisting of diverse high-speed modules

To address this, current approaches (referred to as conventional approach in this thesis) adopt a local model order reduction (LMOR) strategy and then carry out transient analysis of above described linear subnetworks. This involves: (a) individually reducing the large RLC network, distributed transmission lines (and all other components which can be put in the formulation suitable for PRIMA), to state-space macromodels using first-level reduction algorithms such as PRIMA, (b) performing a second-level of reduction individually on the resulting reduced models and also on all other embedded state-space modules corresponding to the networks described by tabulated data, (c) linking the resulting locally-reduced individual compact state-space models and perform the transient analysis in the presence of external terminations/circuitry. However, this process could still be 
$\mathrm{CPU}$ intensive, since the concatenation of several compact state-space systems may not be optimum enough for fast transient analysis.

In order to address the above difficulties, a new methodology for the computation of a single passive compact macromodel for the given linear subnetwork consisting of both RLC elements and multiple embedded state-space modules is presented (this scheme will be henceforth referred to as global model-order reduction (GMOR)), with the following steps. (i) In the first step, simultaneously reduce the entire network containing large number of RLC lumped elements as well as multiple embedded state-space systems, using the first-level reduction algorithm (PRIMA). (ii) In the second step, apply second-level passive reduction to achieve a single compact macromodel. For this purpose, an efficient algorithm is presented, which reduces the total cost of the second-level reduction using PRTBR approach [72], [73], by half.

The following section describes the limitation of PRIMA type of algorithm to include embedded state-space systems. The proposed algorithm (PRESS), which is the first step of the GMOR, is presented in Section 7.2, while the efficient second-level reduction methodology is presented in Section 7.4. Finally, a comparison of the performance of the conventional LMOR and the proposed GMOR strategy is made in Section 7.6.

\subsection{Limitation of PRIMA Type of First-level Algorithms}

In this section, we discuss the limitation of PRIMA algorithm in preserving the passivity of the reduced model, when applied on the systems consisting of embedded passive state-space systems and RLC elements. 
Consider an embedded linear module described by passive state-space equations as

$$
\begin{aligned}
\dot{\boldsymbol{x}}(t) & =\boldsymbol{A} \boldsymbol{x}(t)+\boldsymbol{B} \boldsymbol{i}(t) \\
\boldsymbol{v}(t) & =\boldsymbol{C} \boldsymbol{x}(t)+\boldsymbol{D} \boldsymbol{i}(t)
\end{aligned}
$$

Without loss of generality, let the inputs be the port currents, represented by vector $\boldsymbol{i}(t)$ and outputs be the port voltages, represented by vector $\boldsymbol{v}(t)$. Let $n$ be the total number of state variables, $h$ be the number of ports, and the dimensions of matrices be as follows. $\boldsymbol{A} \in \mathfrak{R}^{n \times n}, \boldsymbol{B} \in \mathfrak{R}^{n \times h}, \boldsymbol{C} \in \mathfrak{R}^{h \times n}, \boldsymbol{D} \in \mathfrak{R}^{h \times h}$ and $\boldsymbol{x}(t) \in \mathfrak{R}^{n}$. Some examples of such linear subnetworks include the systems which result from passive rational approximation of tabulated data [27] - [54], second-level reduced models [73] etc.

Next, the state-space system of (7.1) - (7.2) can be included in the time-domain MNA equations of lumped RLC elements (3.1) as

$$
\begin{gathered}
\boldsymbol{C}_{\phi} \dot{v}_{\phi}(t)+\boldsymbol{G}_{\phi} \boldsymbol{v}_{\phi}(t)+\boldsymbol{L i}(t)=\boldsymbol{b}_{\phi} \boldsymbol{v}_{p}(t) \\
\boldsymbol{i}_{p}(t)=\boldsymbol{b}_{\phi}^{T} \boldsymbol{v}_{\phi}(t)
\end{gathered}
$$

where

- $\phi$ is the domain of lumped part of the network,

- $L=\left[l_{i, j}\right]$ with elements $l_{i, j} \in\{0,1\}$ where $i \in\left\{1, \ldots, N_{\phi}\right\}, j \in\{1, \ldots, h\}$ with a maximum of one nonzero in each row or column, is a selector matrix that maps the port current vector $\boldsymbol{i}(t) \in \mathfrak{R}^{h}$ of the embedded module, into the node space $\Re^{N \phi}$ of the linear subnetwork $\phi$. Also, port voltage vector $v(t) \in \Re^{h}$ of embedded module is related to $\boldsymbol{v}_{\phi}(t) \in \Re^{N}$ as $\boldsymbol{v}(t)=\boldsymbol{L}^{T} \boldsymbol{v}_{\phi}(t)$. 
Combining (7.1) - (7.3), we can write the augmented set of equations as

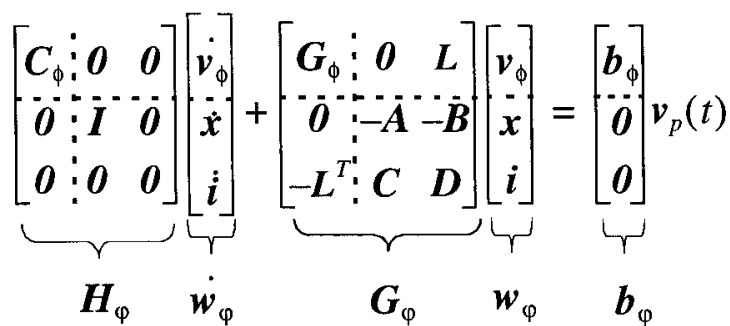

$$
\begin{aligned}
& \boldsymbol{i}_{p}=\boldsymbol{b}_{\varphi}^{T} \boldsymbol{w}_{\varphi}
\end{aligned}
$$

It is to be noticed that, the augmented set of equations (7.4) (shown above for a single embedded state-space system) can be easily extended to handle multiple embedded state-space modules. If PRIMA like algorithm is used on (7.4), then there is no guarantee that the reduced model will be passive. This is because, although the matrices $\boldsymbol{G}_{\phi}, \boldsymbol{C}_{\phi}$ and $\boldsymbol{b}_{\phi}$ are formulated as per (3.2), the unified matrix $\boldsymbol{G}_{\varphi}$ does not guarantee passive reduction. This issue is illustrated through an example, given below.

Consider a 2-port linear subnetwork (Fig. 7.2) with RLC lumped components and an embedded passive 2-port state-space module $(\boldsymbol{A}, \boldsymbol{B}, \boldsymbol{C}, \boldsymbol{D})$ of order 4 . The unified formulation of (7.4) resulted in a MNA of order 14 and the corresponding matrices are given below.

$$
\boldsymbol{A}=\left[\begin{array}{cccc}
-1 & 0 & 0 & 0 \\
0 & -1 & 0 & 0 \\
0 & 0 & -2 & 0 \\
0 & 0 & 0 & -2
\end{array}\right] ; \quad \boldsymbol{B}=\left[\begin{array}{ll}
1 & 0 \\
0 & 1 \\
1 & 0 \\
0 & 1
\end{array}\right] ; \quad \boldsymbol{C}=\left[\begin{array}{cccc}
0.002 & 0.001 & 0.0025 & 0.0015 \\
0.001 & 0.0021 & 0.0015 & 0.0027
\end{array}\right] ; \quad \boldsymbol{D}=\left[\begin{array}{cc}
0.001 & 0 \\
0 & 0.001
\end{array}\right]
$$




$$
\boldsymbol{C}_{\phi}=\left[\begin{array}{cccccccc}
0 & 0 & 0 & 0 & 0 & 0 & 0 & 0 \\
0 & C_{1} & 0 & 0 & 0 & 0 & 0 & 0 \\
0 & 0 & 0 & 0 & 0 & 0 & 0 & 0 \\
0 & 0 & 0 & C_{2} & -C_{2} & 0 & 0 & 0 \\
0 & 0 & 0 & -C_{2} & C_{2} & 0 & 0 & 0 \\
0 & 0 & 0 & 0 & 0 & L_{1} & 0 & 0 \\
0 & 0 & 0 & 0 & 0 & 0 & 0 & 0 \\
0 & 0 & 0 & 0 & 0 & 0 & 0 & 0
\end{array}\right] ; \quad \boldsymbol{G}_{\phi}=\left[\begin{array}{cccccccc}
g_{1} & -g_{1} & 0 & 0 & 0 & 0 & 1 & 0 \\
-g_{1} & g_{1} & 0 & 0 & 0 & 1 & 0 & 0 \\
0 & 0 & g_{2} & 0 & 0 & -1 & 0 & 0 \\
0 & 0 & 0 & g_{3} & -g_{3} & 0 & 0 & 0 \\
0 & 0 & 0 & -g_{3}\left(g_{3}+g_{4}\right) & 0 & 0 & 1 \\
0 & -1 & 1 & 0 & 0 & 0 & 0 & 0 \\
-1 & 0 & 0 & 0 & 0 & 0 & 0 & 0 \\
0 & 0 & 0 & 0 & -1 & 0 & 0 & 0
\end{array}\right] ; \quad \boldsymbol{L}=\left[\begin{array}{cc}
0 & 0 \\
0 & 0 \\
1 & 0 \\
0 & 1 \\
0 & 0 \\
0 & 0 \\
0 & 0 \\
0 & 0
\end{array}\right] ; \quad \boldsymbol{b}_{\phi}=\left[\begin{array}{cc}
0 & 0 \\
0 & 0 \\
0 & 0 \\
0 & 0 \\
0 & 0 \\
0 & 0 \\
-1 & 0 \\
0 & -1
\end{array}\right]
$$

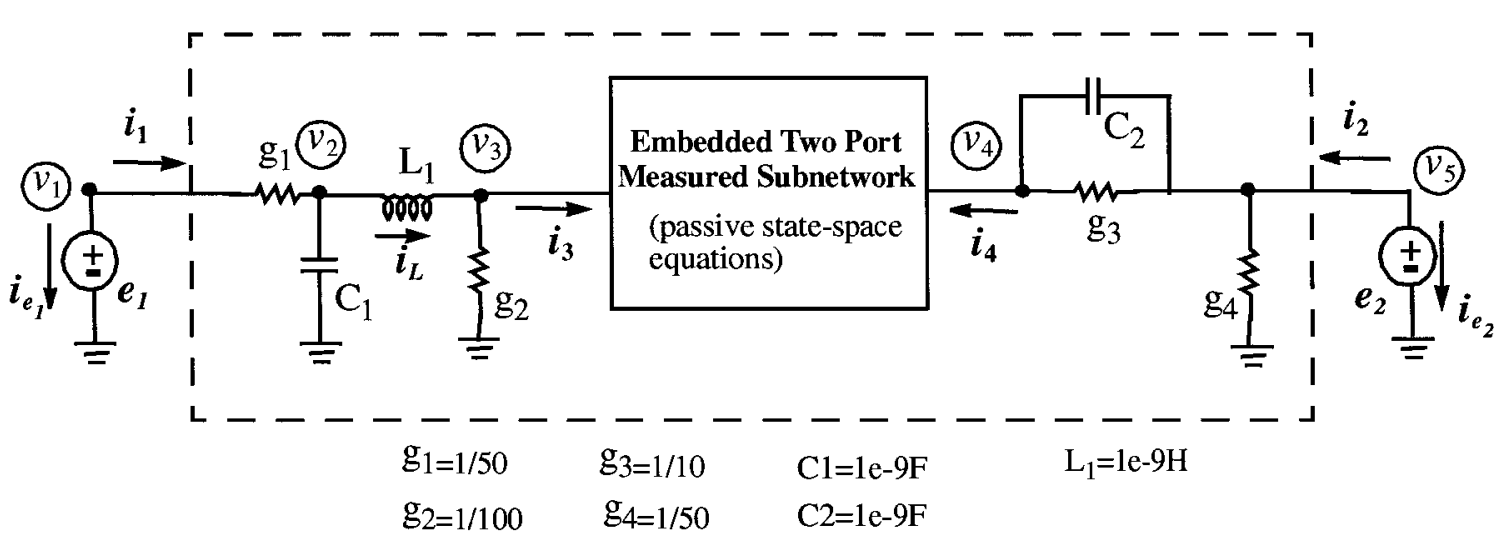

Fig. 7.2. RLC circuit with an embedded state-space module

Next, the above set of equations are reduced to a system of order 6 using congruence transforms as suggested in (3.8) - (3.9). However, it is found that the reduced system is nonpassive. Fig. 7.3 shows the plot of the eigenvalue of realpart-admittance matrix of the original and reduced system. As seen, the original system has positive eigenvalue spectrum, whereas the reduced system contains some negative eigenvalue spectrum, which implies that the reduced system is non-passive [85]. To overcome this difficulty, a new algorithm is presented to include embedded state-space systems while ensuring the passivity of the reduced model. 


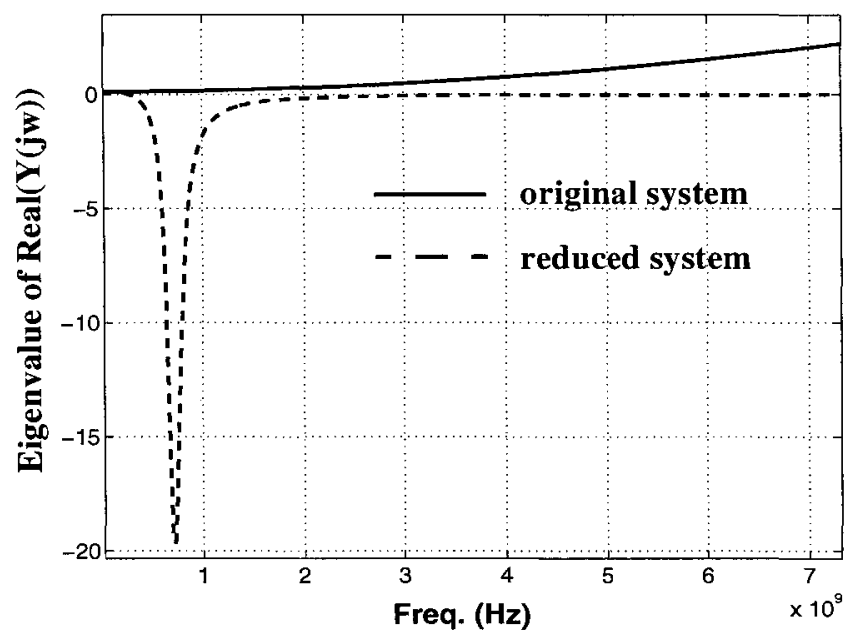

Fig. 7.3. Eigenvalue of $\operatorname{Real}(\boldsymbol{Y}(j \omega)) \mathrm{v} / \mathrm{s}$ frequency

\subsection{Proposed Reduction of RLC Interconnects with Embedded State-Space Systems (PRESS)}

This section presents the proposed algorithm for passive reduction of RLC interconnect networks with embedded passive state-space systems. For the purpose of simplicity of presentation, the discussion given below corresponds to the case of RLC circuits with a single embedded state-space system. The discussion can be easily extended to the case of multiple embedded state-space systems.

\subsubsection{Formulation of Unified Network Equations}

Consider the case of a single $h$-port embedded state-space module described by (7.1) - (7.2), with $n$-states. In order to ensure the passivity of the reduced-order 
model, the following formulation is used. Pre-multiplying (7.1) by a real matrix, $\boldsymbol{P} \in \mathfrak{R}^{n \times n}=\boldsymbol{P}^{T} \geq 0$ we can write

$$
\begin{gathered}
\boldsymbol{P} \dot{x}(t)=\boldsymbol{P A x}(t)+\boldsymbol{P B} \boldsymbol{i}(t) \\
v(t)=\boldsymbol{C} \boldsymbol{x}(t)+\boldsymbol{D i}(t)
\end{gathered}
$$

Using (7.5) - (7.6), the unified network equation (7.4) can be re-written as

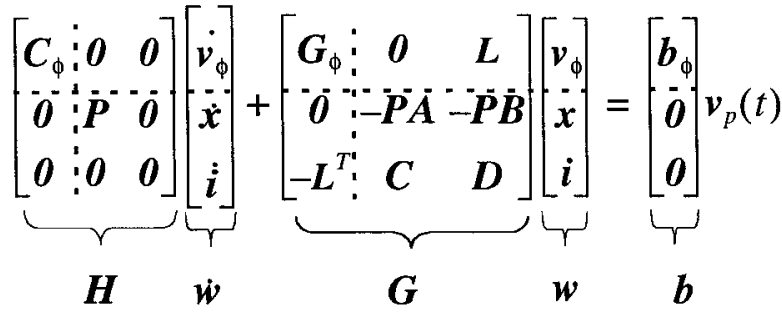

$$
\begin{aligned}
& \boldsymbol{i}_{p}=\boldsymbol{b}^{T} \boldsymbol{w}
\end{aligned}
$$

Let the total number of MNA variables in the above formulation be $m$, such that $\boldsymbol{H}, \boldsymbol{G} \in \mathfrak{R}^{m \times m}, \boldsymbol{w} \in \mathfrak{R}^{m}$ and $\boldsymbol{b} \in \mathfrak{R}^{m \times p}$. It should be noted that, generally the size of the embedded state-space system $(n)$ is much smaller than that of the RLC circuit $N_{\phi}$, i.e. $n \ll N_{\phi}$. Typically $n$ is of the order of few hundreds while $N_{\phi}$ is of the order of thousands. Hence, even though the state-matrix of (7.1) becomes dense after multiplication by matrix $\boldsymbol{P}$ in (7.5), its impact on the overall computational cost involved in the solution of the unified system (7.7) is minimal.

Matrix $\boldsymbol{P}=\boldsymbol{P}^{T} \geq 0$ is obtained as a solution of the following Linear Matrix Inequality (LMI) [70] 


$$
\left[\begin{array}{cc}
-\boldsymbol{A}^{T} \boldsymbol{P}-\boldsymbol{P A} & -\boldsymbol{P B}+\boldsymbol{C}^{T} \\
-\boldsymbol{B}^{T} \boldsymbol{P}+\boldsymbol{C} & \boldsymbol{D}+\boldsymbol{D}^{T}
\end{array}\right] \geq 0
$$

The above inequality is essentially the condition satisfied by a passive system as per the positive-real lemma [70]. For the given embedded module, if $\boldsymbol{P}$ can be obtained such that $\boldsymbol{P}=\boldsymbol{P}^{T} \geq 0$ and satisfying (7.8), then the passivity of the reduced-order model is guaranteed (proof is given Section 7.2.3). Details of computation of $\boldsymbol{P}$ is given in section Section 7.2.4.

Taking the Laplace transformation of (7.7), the admittance matrix of the system is given as

$$
\boldsymbol{Y}(s)=\boldsymbol{b}^{T}(\boldsymbol{G}+s \boldsymbol{H})^{-1} \boldsymbol{b}
$$

\subsubsection{Passive Model-Order Reduction}

Using the congruence transformation, the original system in (7.7) can be written in the reduced form as

$$
\begin{gathered}
\hat{\boldsymbol{H} \dot{\hat{\boldsymbol{w}}}}+\hat{\boldsymbol{G}} \hat{\boldsymbol{w}}=\hat{\boldsymbol{b}} \boldsymbol{v}_{p} \\
\boldsymbol{i}_{p}=\hat{\boldsymbol{b}}^{T} \hat{\boldsymbol{w}}
\end{gathered}
$$

where $\boldsymbol{w}=\boldsymbol{Q} \hat{\boldsymbol{w}}$ and the reduced order matrices are

$$
\hat{\boldsymbol{H}}=\boldsymbol{Q}^{T} \boldsymbol{H} \boldsymbol{Q} ; \quad \hat{\boldsymbol{G}}=\boldsymbol{Q}^{T} \boldsymbol{G} \boldsymbol{Q} ; \quad \hat{\boldsymbol{b}}=\boldsymbol{Q}^{T} \boldsymbol{b}
$$


Here $\boldsymbol{Q} \in \mathfrak{R}^{m \times q}$ is an orthonormal matrix spanning the Krylov subspace $K r\left(-\boldsymbol{G}^{-1} \boldsymbol{H}, \boldsymbol{G}^{-1} \boldsymbol{b}, q\right)$ and $q \ll m$ is the size of the reduced order system in (7.11). From (7.10), the reduced order admittance matrix can be obtained as

$$
\hat{\boldsymbol{Y}}(s)=\hat{\boldsymbol{b}}^{T}(\hat{\boldsymbol{G}}+s \hat{\boldsymbol{H}})^{-1} \hat{\boldsymbol{b}}
$$

It has been shown in [13] that the reduced system (7.12) matches the first $\lfloor q / p\rfloor$ moments of the original system in (7.7). A mathematical proof of the passivity preservation of the reduced model from the proposed algorithm is given in the following section.

\subsubsection{Proof of Passivity Preservation}

A network with admittance matrix represented by $\boldsymbol{Y}(s)$ is passive iff [85]:

(C1) $\left.\boldsymbol{Y}_{\left(s^{*}\right)}\right) \boldsymbol{Y}^{*}(s)$ where '*' is the complex conjugate operator.

(C2) $\boldsymbol{Y}(s)$ is a positive real (PR) matrix, that is the product $z^{* T}\left[\boldsymbol{Y}^{T}\left(s^{*}\right)+\boldsymbol{Y}(s)\right] z \geq 0$ for all complex values of $s$ with $\operatorname{Re}(s)>0$ and any arbitrary vector $z$.

For the reduced-order model (7.12), condition (C1) is satisfied because the reduced matrices $\hat{\boldsymbol{G}}, \hat{\boldsymbol{H}}, \hat{\boldsymbol{b}}$ are real, since the transformation matrix $\boldsymbol{Q}$ is real. Using the formulation of the original system as in (7.7), condition (C2) can be proved for the reduced system as follows. Condition (C2) can be expressed using (7.12) as 


$$
\begin{aligned}
z^{* T}\left[\hat{\boldsymbol{Y}}^{T}\left(s^{*}\right)+\hat{\boldsymbol{Y}}(s)\right] z= & z^{* T}\left[\hat{\boldsymbol{b}}^{T}\left(\hat{\boldsymbol{G}}+s^{*} \hat{\boldsymbol{H}}\right)^{-T} \hat{\boldsymbol{b}}+\hat{\boldsymbol{b}}^{T}(\hat{\boldsymbol{G}}+s \hat{\boldsymbol{H}})^{-1} \hat{\boldsymbol{b}}\right] z \\
& =z^{* T} \hat{\boldsymbol{b}}^{T}\left[\left(\hat{\boldsymbol{G}}+s^{*} \hat{\boldsymbol{H}}\right)^{-T}+(\hat{\boldsymbol{G}}+s \hat{\boldsymbol{H}})^{-1}\right] \hat{\boldsymbol{b}} z \\
& =z^{*{ }^{*}} \hat{\boldsymbol{b}}^{T}(\hat{\boldsymbol{G}}+s \hat{\boldsymbol{H}})^{-1}\left[(\hat{\boldsymbol{G}}+s \hat{\boldsymbol{H}})+\left(\hat{\boldsymbol{G}}+s^{*} \hat{\boldsymbol{H}}\right)^{T}\right]\left(\hat{\boldsymbol{G}}+s^{*} \hat{\boldsymbol{H}}\right)^{-T} \hat{\boldsymbol{b}} z
\end{aligned}
$$

Substituting $\boldsymbol{k}=\left(\hat{\boldsymbol{G}}+s^{*} \hat{\boldsymbol{H}}\right)^{-T} \hat{\boldsymbol{b}} \boldsymbol{z}$ and $s=\sigma+j \omega,(7.13)$ can be expressed as

$$
\begin{gathered}
z^{* T}\left[\hat{\boldsymbol{Y}}^{T}\left(s^{*}\right)+\hat{\boldsymbol{Y}}(s)\right] z=\boldsymbol{k}^{* T}\left[(\hat{\boldsymbol{G}}+(\sigma+j \omega) \hat{\boldsymbol{H}})+(\hat{\boldsymbol{G}}+(\sigma-j \omega) \hat{\boldsymbol{H}})^{T}\right] \boldsymbol{k} \\
=\boldsymbol{k}^{* T}\left[\hat{\boldsymbol{G}}+\hat{\boldsymbol{G}}^{T}+\sigma\left(\hat{\boldsymbol{H}}+\hat{\boldsymbol{H}}^{T}\right)+j \omega\left(\hat{\boldsymbol{H}}-\hat{\boldsymbol{H}}^{T}\right)\right] \boldsymbol{k}
\end{gathered}
$$

Substituting for $\hat{\boldsymbol{G}}$ and $\hat{\boldsymbol{H}}$ from (7.11), (7.14) can be expressed as

$$
\boldsymbol{z}^{* T}\left[\hat{\boldsymbol{Y}}^{T}\left(s^{*}\right)+\hat{\boldsymbol{Y}}(s)\right] z=\boldsymbol{k}^{* T}\left[\boldsymbol{Q}^{T} \boldsymbol{G} \boldsymbol{Q}+\boldsymbol{Q}^{T} \boldsymbol{G}^{T} \boldsymbol{Q}+\sigma\left(\boldsymbol{Q}^{T} \boldsymbol{H} \boldsymbol{Q}+\boldsymbol{Q}^{T} \boldsymbol{H}^{T} \boldsymbol{Q}\right)+j \omega\left(\boldsymbol{Q}^{T} \boldsymbol{H} \boldsymbol{Q}-\boldsymbol{Q}^{T} \boldsymbol{H}^{T} \boldsymbol{Q}\right)\right] \boldsymbol{k}
$$

Noting from (7.7) that the matrix $\boldsymbol{H}$ is symmetric and nonnegative definite (since both $\boldsymbol{P}$ (obtained by solving (7.8)) and $\boldsymbol{C}_{\phi}$ (formulated as per (3.2)) are symmetric and nonnegative definite), (7.15) can be simplified as

$$
\boldsymbol{z}^{* T}\left[\hat{\boldsymbol{Y}}^{T}\left(s^{*}\right)+\hat{\boldsymbol{Y}}(s)\right] z=\boldsymbol{k}^{* T} \boldsymbol{Q}^{T}\left[\boldsymbol{G}+\boldsymbol{G}^{T}+\sigma\left(\boldsymbol{H}+\boldsymbol{H}^{T}\right)\right] \boldsymbol{Q} \boldsymbol{k}
$$

Substituting $\hat{\boldsymbol{k}}=\boldsymbol{Q} \boldsymbol{k},(7.16)$ can be written as

$$
\begin{aligned}
\boldsymbol{z}^{* T}\left[\hat{\boldsymbol{Y}}^{T}\left(s^{*}\right)+\hat{\boldsymbol{Y}}(s)\right] z & =\hat{\boldsymbol{k}}^{* T}\left[\boldsymbol{G}+\boldsymbol{G}^{T}+\sigma\left(\boldsymbol{H}+\boldsymbol{H}^{T}\right)\right] \hat{\boldsymbol{k}} & & \psi_{1}=\hat{\boldsymbol{k}}^{*_{T}}\left[\sigma\left(\boldsymbol{H}+\boldsymbol{H}^{T}\right)\right] \hat{\boldsymbol{k}} \\
& =\psi_{1}+\psi_{2} & & \psi_{2}=\hat{\boldsymbol{k}}^{{ }^{*} T}\left[\boldsymbol{G}+\boldsymbol{G}^{T}\right] \hat{\boldsymbol{k}}
\end{aligned}
$$

Since $\boldsymbol{H}$ is symmetric and nonnegative definite, it can be concluded that

$$
\psi_{1}=\hat{\boldsymbol{k}}^{* T}\left[\sigma\left(\boldsymbol{H}+\boldsymbol{H}^{T}\right)\right] \hat{\boldsymbol{k}}=2 \sigma \hat{\boldsymbol{k}}^{* T} \boldsymbol{H} \hat{\boldsymbol{k}} \geq 0
$$


for any $\operatorname{Re}(s)=\sigma>0$ and complex vector $\hat{\boldsymbol{k}}$. Next, using the definition of $\boldsymbol{G}$ from (7.7), $\psi_{2}$ can be expressed as

$$
\begin{aligned}
\psi_{2}=\hat{\boldsymbol{k}}^{* T}\left[\boldsymbol{G}+\boldsymbol{G}^{T}\right] \hat{\boldsymbol{k}} & =\hat{\boldsymbol{k}}^{* T}\left(\left[\begin{array}{ccc}
\boldsymbol{G}_{\phi} & \boldsymbol{0} & \boldsymbol{L} \\
\boldsymbol{0} & -\boldsymbol{P A} & -\boldsymbol{P B} \\
-\boldsymbol{L}^{T} & \boldsymbol{C} & \boldsymbol{D}
\end{array}\right]+\left[\begin{array}{ccc}
\boldsymbol{G}_{\phi} & \boldsymbol{0} & \boldsymbol{L} \\
\boldsymbol{0} & -\boldsymbol{P A} & -\boldsymbol{P B} \\
-\boldsymbol{L}^{T} & \boldsymbol{C} & \boldsymbol{D}
\end{array}\right]\right) \hat{\boldsymbol{k}} \\
& =\hat{\boldsymbol{k}}^{* T}\left[\begin{array}{ccc}
\boldsymbol{G}_{\phi}+\boldsymbol{G}_{\phi}^{T} & \boldsymbol{0} & \boldsymbol{0} \\
\boldsymbol{0} & -\boldsymbol{A}^{T} \boldsymbol{P}-\boldsymbol{P A} & -\boldsymbol{P} \boldsymbol{B}+\boldsymbol{C}^{T} \\
\boldsymbol{0} & -\boldsymbol{B}^{T} \boldsymbol{P}+\boldsymbol{C} & \boldsymbol{D}+\boldsymbol{D}^{T}
\end{array}\right] \hat{\boldsymbol{k}}
\end{aligned}
$$

Since, $\boldsymbol{G}_{\phi}$ is the conductance matrix corresponding to the lumped circuit elements (formed as per (3.2)), we have

$$
\left(\boldsymbol{G}_{\phi}+\boldsymbol{G}_{\phi}^{T}\right) \geq 0
$$

Also note that, as per the positive-real lemma [70], there exists a matrix $\boldsymbol{P}=\boldsymbol{P}^{T} \geq 0$ for a passive state-space system, satisfying (7.8). Using these, it can be inferred from (7.19) that

$$
\psi_{2}=\hat{\boldsymbol{k}}^{* T}\left[\boldsymbol{G}+\boldsymbol{G}^{T}\right] \hat{\boldsymbol{k}}=\hat{\boldsymbol{k}}^{*_{T}^{T}}\left[\begin{array}{ccc}
\boldsymbol{G}_{\phi}+\boldsymbol{G}_{\phi}^{T} & \boldsymbol{0} & \boldsymbol{0} \\
\boldsymbol{0} & -\boldsymbol{A}^{T} \boldsymbol{P}-\boldsymbol{P A} & -\boldsymbol{P} \boldsymbol{B}+\boldsymbol{C}^{T} \\
\boldsymbol{0} & -\boldsymbol{B}^{T} \boldsymbol{P}+\boldsymbol{C} & \boldsymbol{D}+\boldsymbol{D}^{T}
\end{array}\right] 0
$$

Using (7.17), (7.18) and (7.21) it can be easily concluded that the passivity condition (C2) is satisfied for the reduced-order model (7.11). 
Next, to demonstrate the passivity of the reduced model numerically, consider the example described in Section 7.1 (Fig. 7.2). Formulation according to (7.7) resulted in a set of unified equations of order 14. For this example, matrix $\boldsymbol{P}$ satisfying (7.8) is given below:

$$
\boldsymbol{P}=10^{-4}\left[\begin{array}{lllll}
4.0676557 & 2.6635249 & 3.9456769 & 2.9746138 \\
2.6635249 & 4.315911 & 2.9558487 & 4.2967426 \\
3.9456769 & 2.9558487 & 4.3663801 & 3.6285253 \\
2.9746138 & 4.2967426 & 3.6285253 & 4.860772
\end{array}\right]
$$

Subsequently, the reduced system of order 6 was obtained using the congruence transformation (7.11). Next, the reduced system is verified for passivity by constructing the corresponding Hamiltonian matrix and checking its eigenvalues. No imaginary eigenvalues were found and hence the macromodel is proved to be passive [70]. This is also numerically demonstrated by plotting the eigenvalue of real part of $\boldsymbol{Y}(j \omega)$ in Fig. 7.4 (which now contains positive eigenvalues).

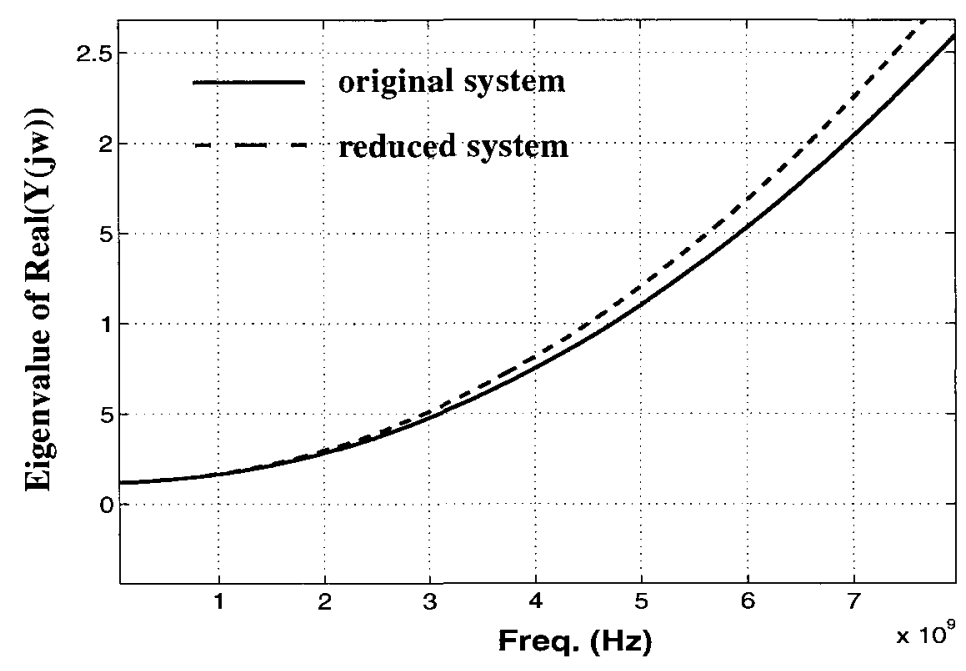

Fig. 7.4. Eigenvalue of $\operatorname{Real}(\boldsymbol{Y}(j \omega)) \mathrm{v} / \mathrm{s}$ frequency 


\subsubsection{Computation of Matrix $P$}

In this section, a brief discussion of computation of real symmetric positive semidefinite matrix $\boldsymbol{P}$ is given. Additional details and related computational considerations can be found in [76], [88]. Assuming that the macromodel represented by (7.1) - (7.2) is passive, then, according to the positive-real lemma there exists a real matrix $\boldsymbol{P}=\boldsymbol{P}^{T} \geq 0$ [70] such that the Linear Matrix Inequality (LMI) given in (7.8) is satisfied. The solution of the inequality (7.8) is related to the solution of the associated riccati equation (ARE)

$$
\boldsymbol{A}^{T} \boldsymbol{P}+\boldsymbol{P A}+\left(\boldsymbol{P B}-\boldsymbol{C}^{T}\right)\left(\boldsymbol{D}+\boldsymbol{D}^{T}\right)^{-1}\left(\boldsymbol{P B}-\boldsymbol{C}^{T}\right)^{T}=\mathbf{0}
$$

Essentially, a solution $\boldsymbol{P}=\boldsymbol{P}^{T} \geq 0$ of (7.23) also satisfies the LMI (7.8) [89]. One of the efficient methods to solve (7.23) is the Schur Method [76], [88]. A summary of the essential steps involved in obtaining the solution is as follows. Noting that a solution of (7.23), satisfying $\boldsymbol{P}=\boldsymbol{P}^{T} \geq 0$ lies in the stable subspace of the following Hamiltonian matrix:

$$
\boldsymbol{M}=\left[\begin{array}{cc}
\boldsymbol{A}-\boldsymbol{B}\left(\boldsymbol{D}+\boldsymbol{D}^{T}\right)^{-1} \boldsymbol{C} & \boldsymbol{B}\left(\boldsymbol{D}+\boldsymbol{D}^{T}\right)^{-1} \boldsymbol{B}^{T} \\
-\boldsymbol{C}^{T}\left(\boldsymbol{D}+\boldsymbol{D}^{T}\right)^{-1} \boldsymbol{C} & -\boldsymbol{A}^{T}+\boldsymbol{C}^{T}\left(\boldsymbol{D}+\boldsymbol{D}^{T}\right)^{-1} \boldsymbol{B}^{T}
\end{array}\right]
$$

and can be obtained as follows. Compute an orthogonal matrix $\boldsymbol{F}$ such that

$$
\boldsymbol{F}^{T} M F=S
$$


where $\quad \boldsymbol{F}=\left[\begin{array}{ll}\boldsymbol{F}_{11} & \boldsymbol{F}_{12} \\ \boldsymbol{F}_{21} & \boldsymbol{F}_{22}\end{array}\right] ; \quad \boldsymbol{F}_{i j} \in \mathfrak{R}^{n \times n}$ and $\boldsymbol{S}=\left[\begin{array}{cc}\boldsymbol{S}_{11} & \boldsymbol{S}_{12} \\ \mathbf{0} & \boldsymbol{S}_{22}\end{array}\right], \quad$ a quasi-upper-triangular matrix with all the eigenvalues of $S_{11}$ lying in the left-half plane. The $n$ Schur vectors comprising $\left[\begin{array}{l}\boldsymbol{F}_{11} \\ \boldsymbol{F}_{21}\end{array}\right]$ span the stable invariant sub-space and the solution of (7.23) is given by $\boldsymbol{P}=\boldsymbol{F}_{21} \boldsymbol{F}_{11}^{-1}$. The computational cost involved in obtaining $\boldsymbol{P}$ is $O\left(n^{3}\right), n$ being the order of the state-space system. It is to be noted from (7.7), that $\boldsymbol{P}$ is needed only for the embedded passive state-space modules, which are usually of relatively low-orders compared to the RLC part of the circuit.

For cases where the state-space system is in the following descriptor form:

$$
\begin{gathered}
\boldsymbol{E} \dot{\boldsymbol{x}}(t)=\boldsymbol{A} \boldsymbol{x}(t)+\boldsymbol{B} \boldsymbol{u}(t) \\
\boldsymbol{y}(t)=\boldsymbol{C} \boldsymbol{x}(t)+D \boldsymbol{u}(t)
\end{gathered}
$$

and $\boldsymbol{E}$ is singular, the methodology based on additive decomposition of the transfer function corresponding to (7.26) - (7.27) can be used [73], [90]. Also, for the case where $\boldsymbol{D}$ is singular or $\boldsymbol{D}=0$, the matrix $\boldsymbol{P}$ can be calculated by using the algorithms such as in [91].

A brief summary of the proposed algorithm PRESS is given in Fig. 7.5 in the form of a pseudocode 
- Step 1: Formulate the MNA equations for the $R L C$ circuit and obtain $C_{\phi}$ and $G_{\phi}$ as per (3.2).

- Step 2: Formulate the Riccati equation (7.23) for each embedded state-space system. Perform Schur decomposition of associated Hamiltonian matrix to obtain the stable invariant subspace. Use this subspace to obtain the respective symmetric positive-definite matrix $\boldsymbol{P}$ satisfying (7.23).

- Step 3: Use matrix $\boldsymbol{P}$ from Step 2 to modify the state-space equation (7.1), to (7.5).

- Step 4: Combine the modified state-space equations (7.5)-(7.6) of embedded passive systems with MNA equations of RLC system to obtain the unified MNA equations of (7.7).

- Step 5: Compute the orthonormal matrix $Q \in \Re^{m \times q}$ spanning the Krylov space $K r\left(-\boldsymbol{G}^{-1} \boldsymbol{H}, \boldsymbol{G}^{-1} \boldsymbol{b}, q\right)$ ( $q$ «m is the size of the reduced order system).

- Step 6: Use this transformation matrix to carry out the passivity preserving first level reduction on the unified system (7.7) by applying the congruent transformations (7.11).

Fig. 7.5. Pseudocode for the proposed global model-order reduction algorithm (PRESS)

\subsection{Numerical Results}

In this section, three examples are presented to demonstrate the validity and efficiency of the proposed algorithm PRESS (presented in the previous section).

\subsubsection{Example 1: Two Port RLC Network with Embedded Subnetwork}

This example further illustrates the concept of the proposed algorithm PRESS for passive reduction in the presence of embedded state-space systems. The same network used in Fig. 7.2 is considered here. The linear measured subnetwork is 
described by a passive state-space system $\boldsymbol{A}, \boldsymbol{B}, \boldsymbol{C}, \boldsymbol{D}$ of order 26 . Using the formulation (7.4) results in a MNA of order 36 . When this system is reduced to a system of order 20 by congruence transformation, it is found that an eigenvalue of $\operatorname{Real}(Y(j \omega))$ of the reduced system is negative, as shown in Fig. 7.6 (in dashed line). This confirms that the reduced system is nonpassive.

Next, the proposed unified formulation using (7.7) (PRESS) is obtained and is reduced to a order of size 20. Proposed reduced-order system is tested for passivity by constructing the corresponding Hamiltonian matrix and checking its eigenvalues. No imaginary eigenvalues were found and hence the macromodel is proved to be passive [70] (also demonstrated numerically in Fig. 7.7, by plotting the eigenvalue spectrum of $\operatorname{Real}(\boldsymbol{Y}(\boldsymbol{j} \omega))$ against frequency).

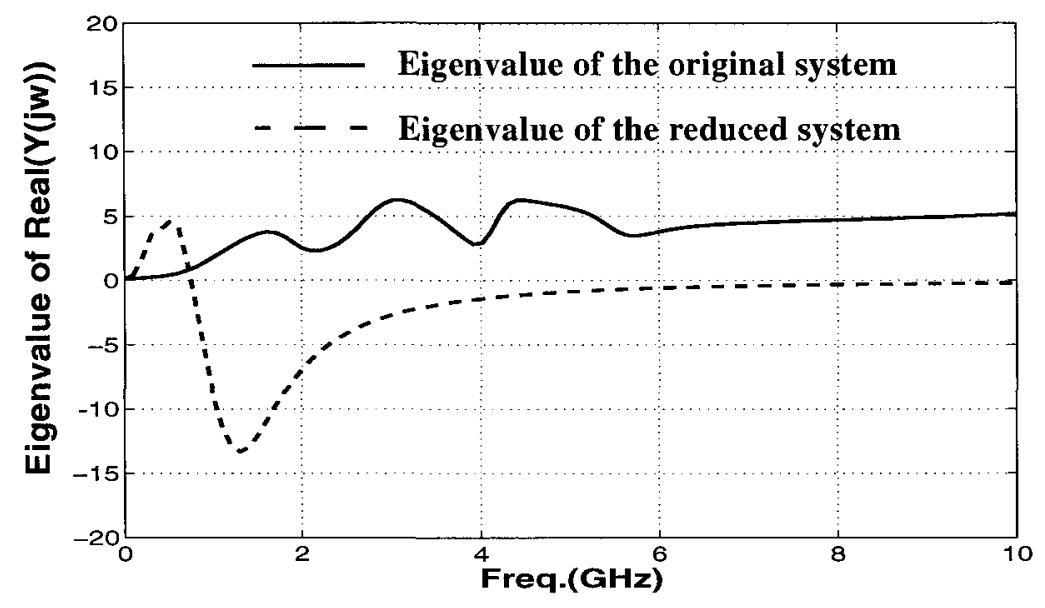

Fig. 7.6. Eigenvalue of $\operatorname{Real}(Y(j \omega))$ - example 1 


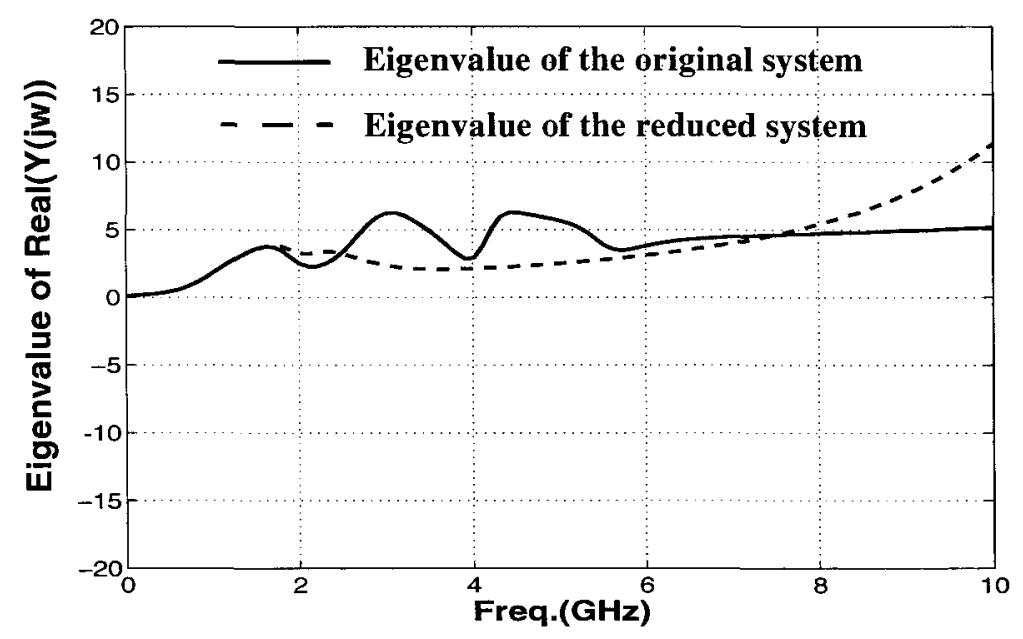

Fig. 7.7. Eigenvalue of $\operatorname{Real}(Y(j \omega))$ - example 1

\subsubsection{Example 2: Two Port RLC Network with Embedded Subnetwork}

In this example, a 2-port linear subnetwork with an embedded 2-port measured module (via), 610 resistors, 600 inductors and 200 capacitors is considered (Fig. 7.8). The passive state-space macromodel for the measured module is obtained using [39], with an order of $45 \times 45$. The overall size of MNA matrices (7.7) (of the lumped circuit with the macromodel of measured module) is $1433 \times 1433$. Using the PRESS algorithm, the reduced macromodel of size $100 \times 100$ was obtained. Fig. 7.9 compares the Y-parameters of the original (unreduced) and reduced systems and they match accurately up to $6 \mathrm{GHz}$. The reduced-order system is tested for passivity by constructing the corresponding Hamiltonian matrix and checking its eigenvalues. No imaginary eigenvalues are found and hence the macromodel is proved to be passive [70] (also demonstrated numerically in Fig. 7.10, by plotting the eigenvalue spectrum of $\operatorname{Real}(Y(j \omega))$ against frequency up to $100 \mathrm{GHz}$ ). 
Next, the reduced system is linked to HSPICE and a non-linear transient analysis is performed, for an input pulse having a rise and fall time of 0.25 ns and pulse width of 5 ns. The results at node $\mathbf{P}_{1}, \mathbf{P}_{2}$ and $\boldsymbol{V}_{\text {out }}$ are shown in Fig. 7.11. For validation purposes, the original system (un-reduced) is also subjected to the transient analysis (using HSPICE) with the similar input and terminations, and the results are compared in Fig. 7.11. As seen, both match accurately. Table 7.1 shows a comparison of the CPU time required for the above transient analysis.

Table. 7.1. Comparison of CPU time in sec. - example 2 (Sun-Blade-100 machine)

\begin{tabular}{|c|c|c|}
\hline Original circuit & PRESS macromodel & Speed-up \\
\hline 29.92 & 2.17 & 14 \\
\hline
\end{tabular}

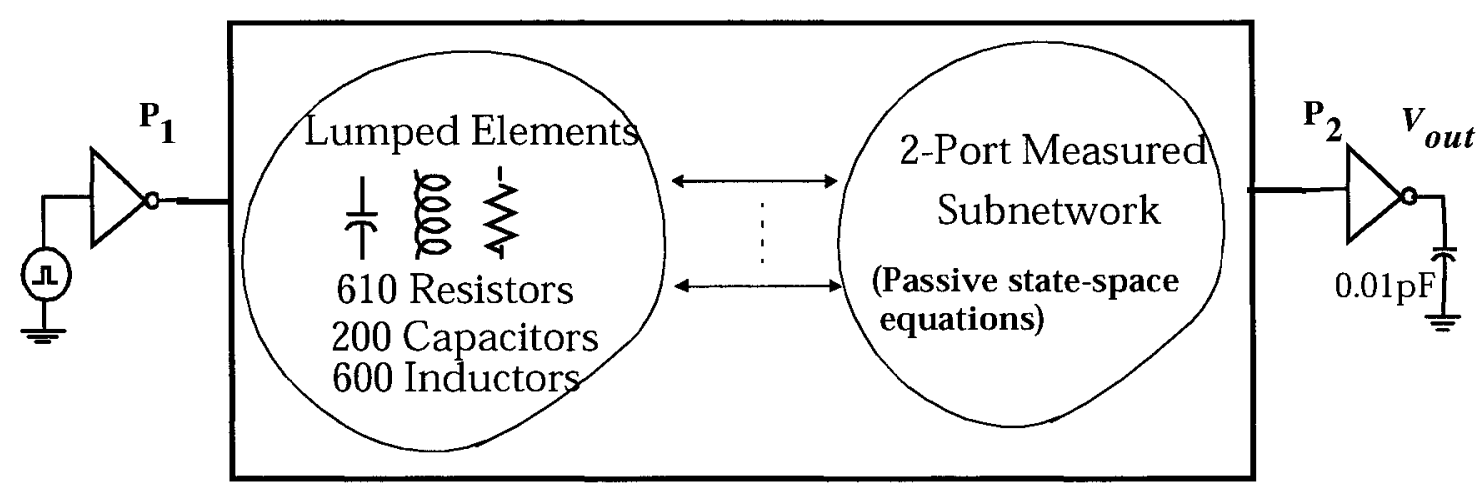

Fig. 7.8. Circuit with lumped components and measured subnetwork - example 2 

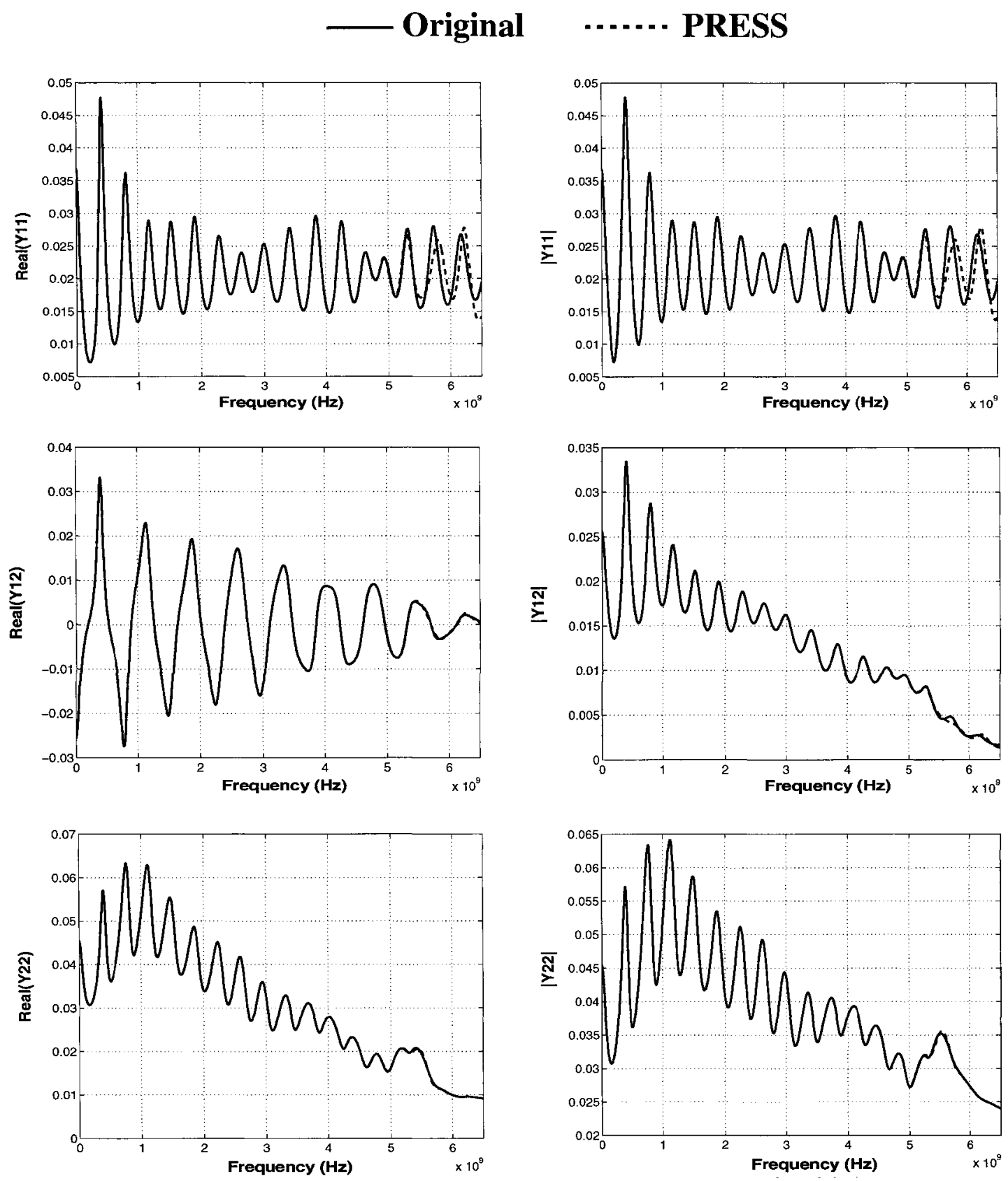

Fig. 7.9. Frequency responses - example 2 

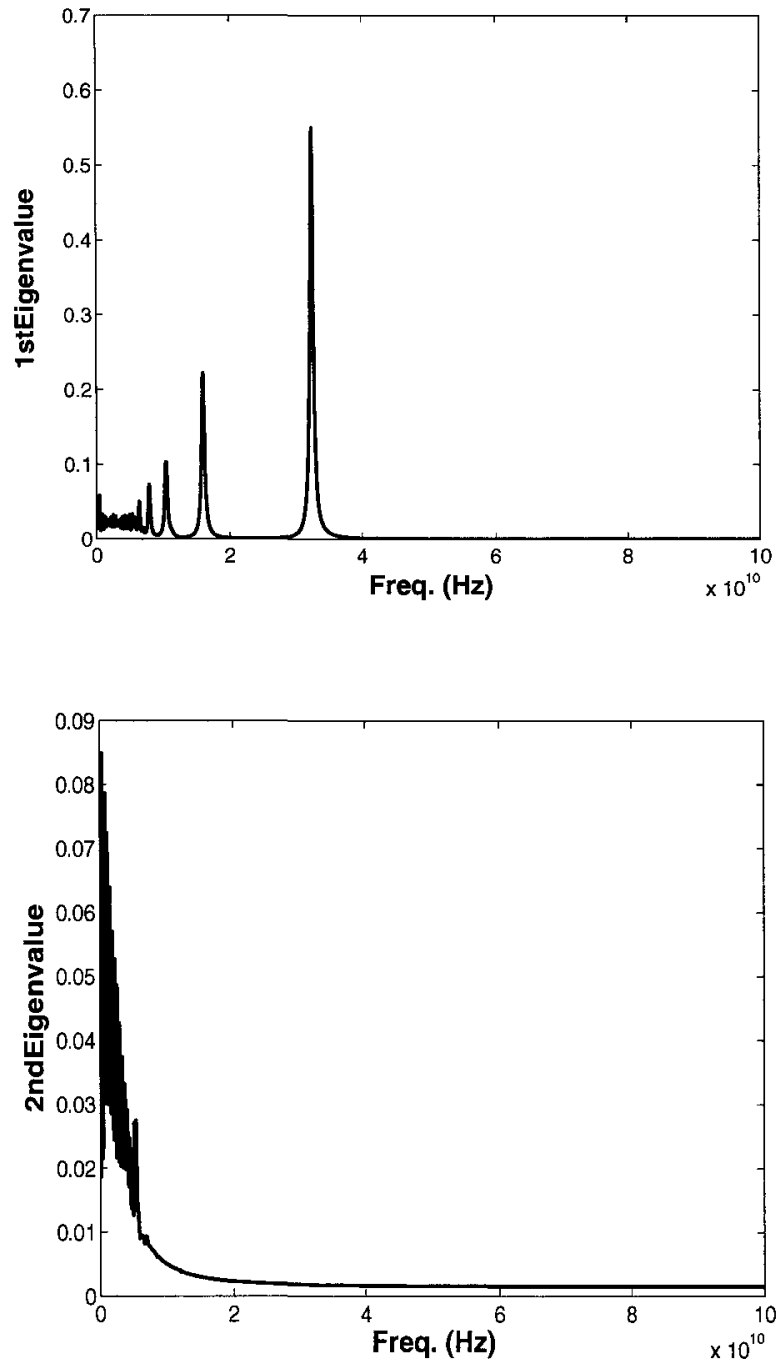

Fig. 7.10. Eigenvalues of $\operatorname{Real}(Y(j \omega)) \mathrm{v} / \mathrm{s}$ frequency for the reduced system example 2 

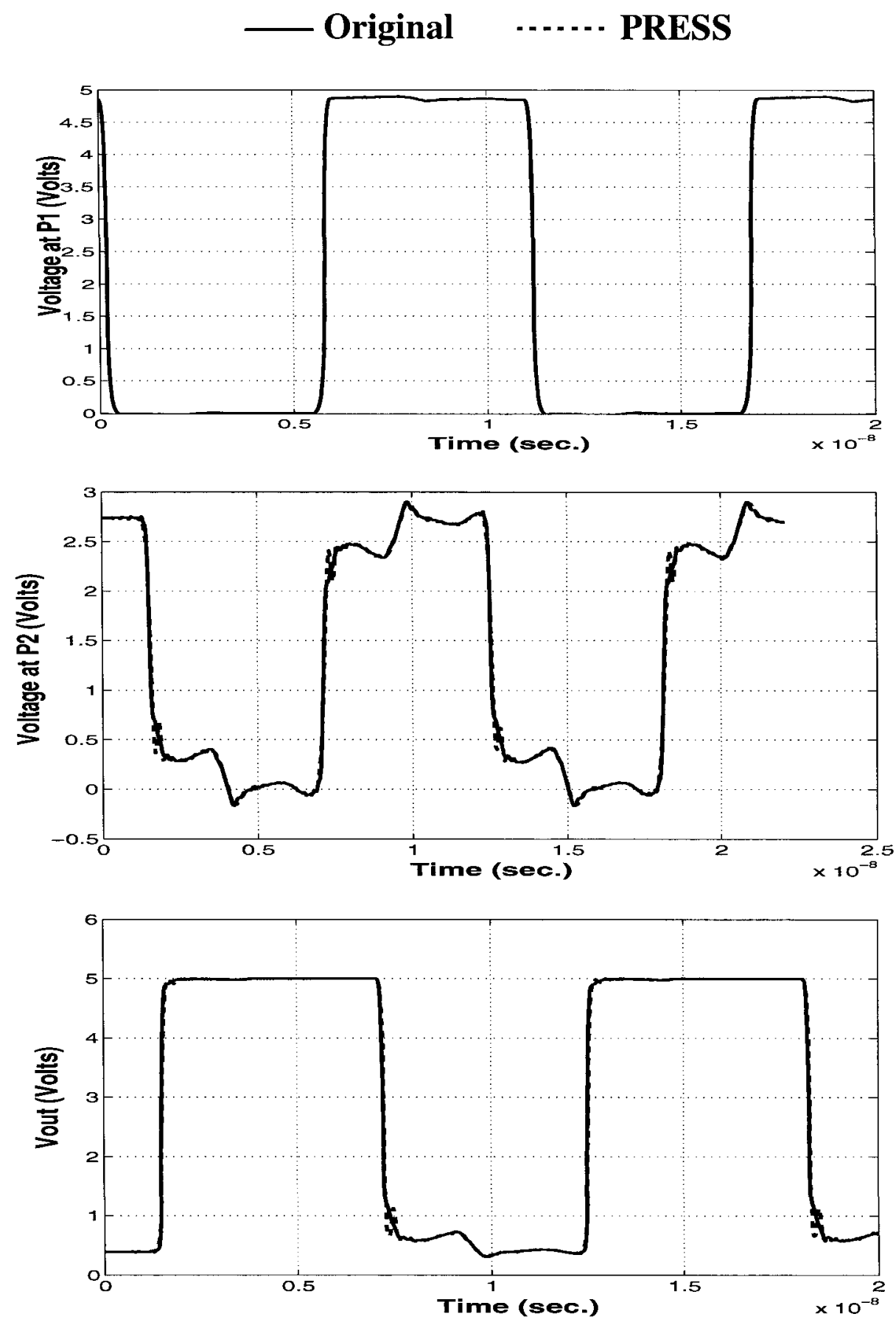

Fig. 7.11. Time domain responses - example 2 


\subsubsection{Example 3: Three Port RLC Network with Embedded State- Space System}

In this example, a 3-port linear subnetwork of Fig. 7.12 is considered. It consists of 4000 resistors, 2000 inductors, 2000 capacitors, and a three-port measured module. A passive state-space macromodel of order $132 \times 132$ is obtained for the measured subnetwork using [39].

The overall size of resulting MNA matrices, using the regular PRIMA formulation (7.4) (including both the lumped circuit and the passive state-space macromodel) was $4142 \times 4142$. This system is reduced to a system of order $192 \times 192$ by congruence transformation and it is found that the reduced system is nonpassive and is confirmed by the plot of $1^{\text {st }}$ eigenvalue of $\operatorname{Real}(\boldsymbol{Y}(j \omega))$ in the Fig. 7.13 (shown in dashed line). Next, using the proposed global model reduction algorithm, PRESS, a reduced-order model with order same as the previous case $(192 \times 192)$ is obtained. The time taken by PRESS algorithm to create the reduced model is 31.2 seconds on a Sun-Blade- 100 machine. The resulting macromodel is tested for passivity by constructing the corresponding Hamiltonian matrix and checking its eigenvalues. No imaginary eigenvalues are found and hence the proposed macromodel is proved to be passive [70] (also demonstrated numerically in Fig. 7.14, by plotting the $1^{\text {st }}$ eigenvalue of $\operatorname{Real}(Y(j \omega))$ against frequency). Fig. 7.15 compares the Y-parameters of the original and the reduced system (PRESS) and they match accurately up to $6.0 \mathrm{GHz}$.

Next, the reduced system is linked to HSPICE and a non-linear transient analysis is performed, for an input pulse having a rise and fall time of 0.1 ns and width of 5 ns. The results at node $\mathbf{P}_{1}, \mathbf{P}_{3}, \boldsymbol{V}_{\text {out } 1}$ and $\boldsymbol{V}_{\text {out }}$ are shown in Fig. 7.16. For validation purposes, the original system is also subjected to the transient analysis 
(using HSPICE) with the similar input and terminations, and the results are compared in Fig. 7.16. As seen, both match accurately. The reduced system from PRESS took 97 seconds, while the original system took 2041 seconds on a SunBlade-100 machine. For a comparative note, we performed the analysis of this system using the LMOR strategy. In this case the transient simulation of the concatenated system of individual reduced models of RLC networks and the state-space model of the measured network required 312 seconds, for providing comparable responses of Fig. 7.16. Table 7.2 summarizes the comparison of CPU times for transient analysis, using various methods described above. The speedup is expected to increase further in the presence of many embedded systems and large number of ports.

Table. 7.2. Comparison of CPU time in sec. - example 3 (Sun-Blade-100 machine)

\begin{tabular}{|c|c|c|}
\hline Original circuit & LMOR & PRESS \\
\hline 2041 & 312 & 97 \\
\hline Speed-up & & 21 \\
\hline
\end{tabular}

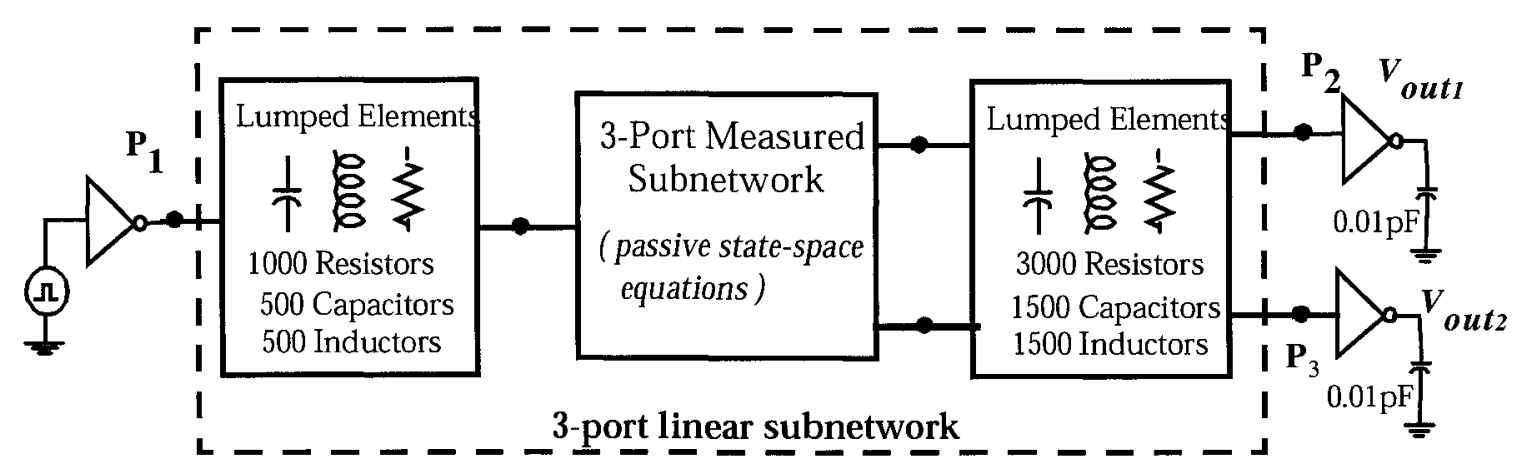

Fig. 7.12. Circuit of Example 3 


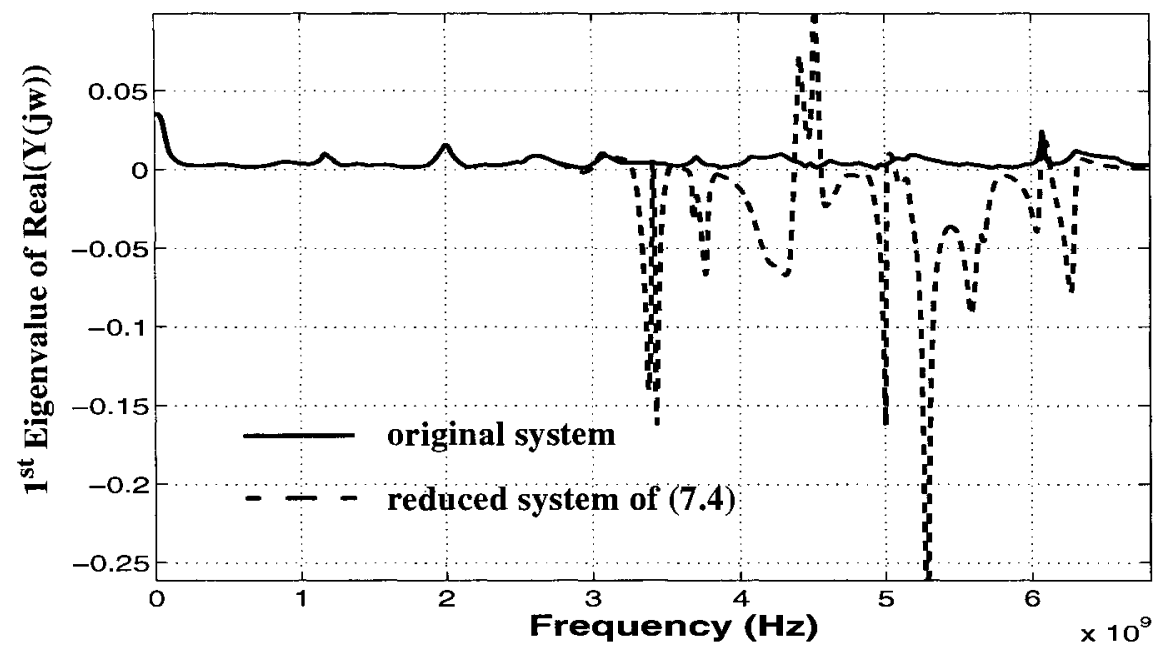

Fig. 7.13. Eigenvalue spectrum of $\operatorname{Real}(Y(j \omega))$ : Original and reduced system of (7.4) - example 3

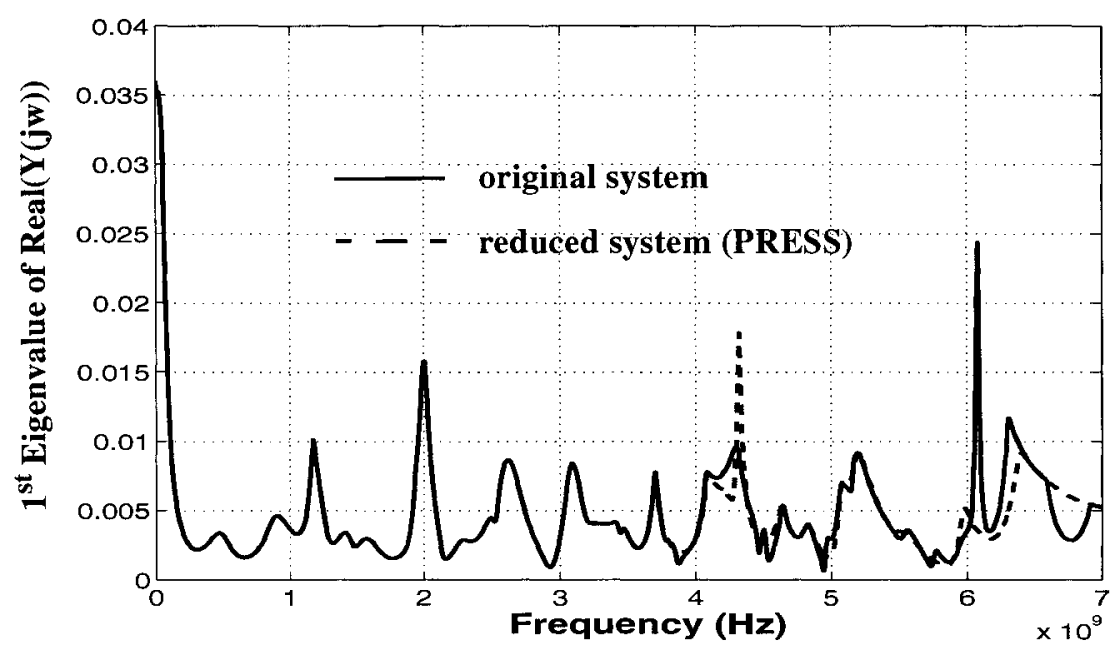

Fig. 7.14. Eigenvalue spectrum of $\operatorname{Real}(Y(j \omega))$ : Original vs. PRESS - example 3 

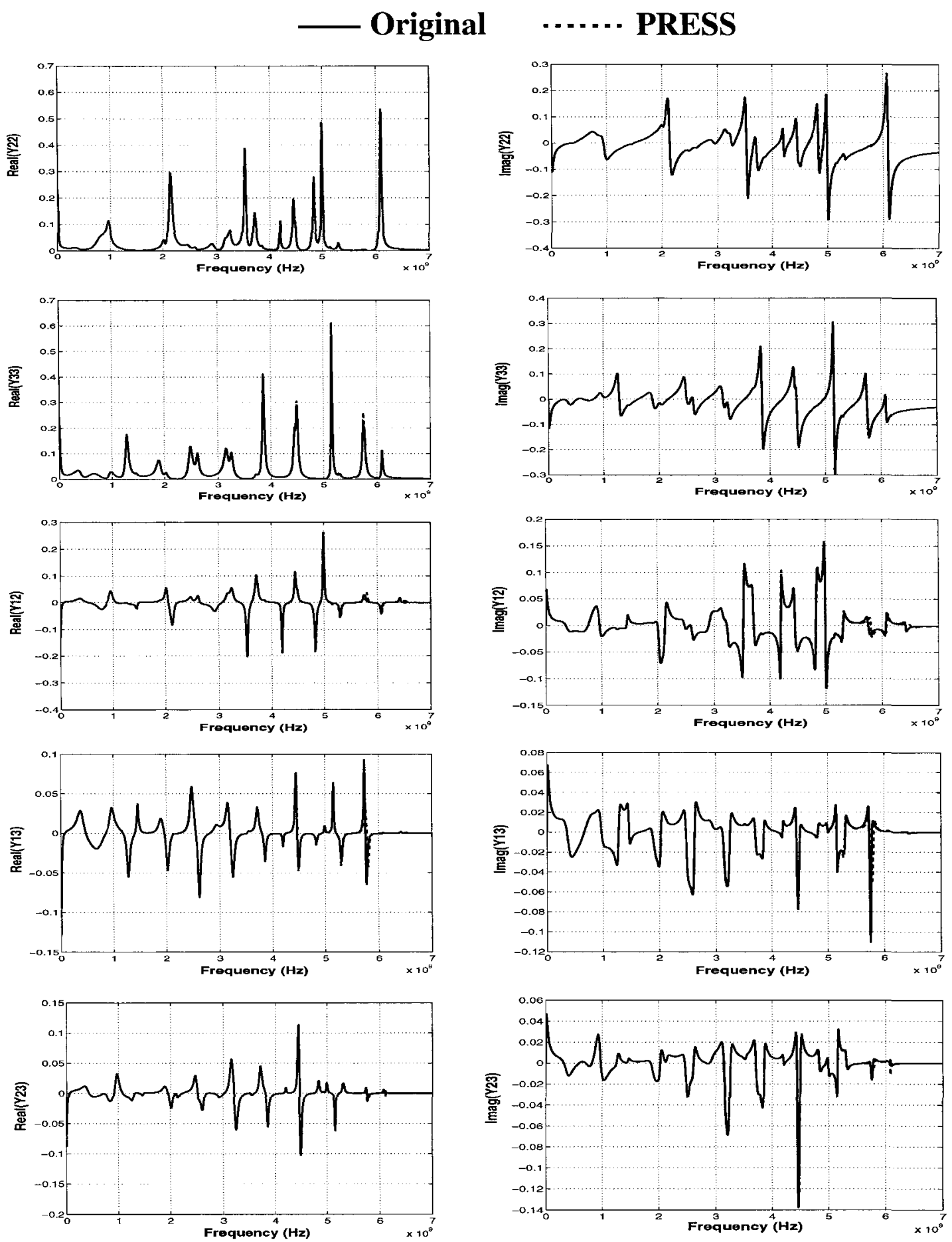

Fig. 7.15. Frequency responses - example 3 

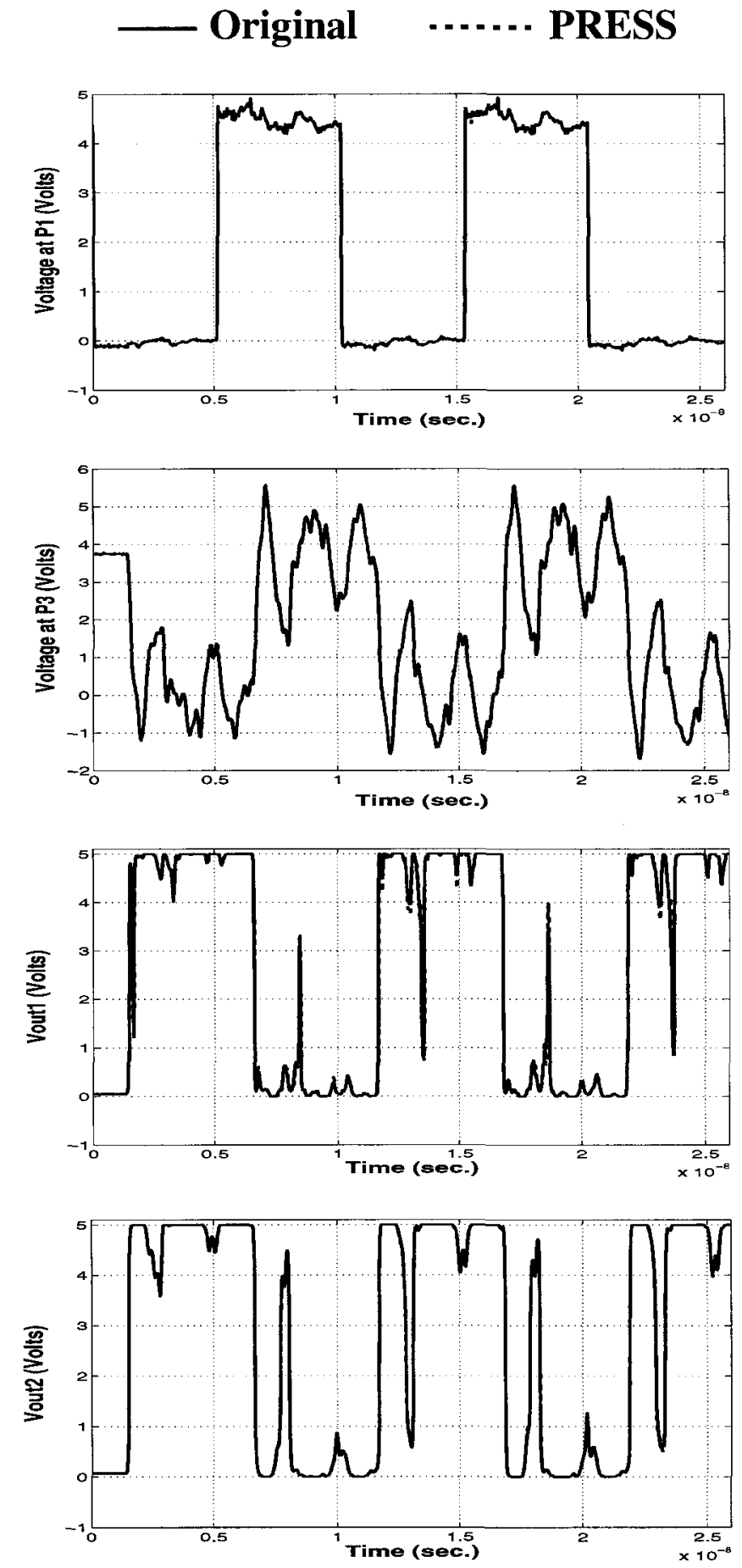

Fig. 7.16. Transient results - example 3 
Recently, it has become apparent that, while Krylov subspace based first-level passive reduction methods are suitable for the analysis of large systems, they may not result in compact models. This led to the focus on second-level reduction algorithms (such as truncated balanced realization (TBR) [71]), described in chapter 3. TBR algorithms are suitable for small systems (with a maximum of few hundred states). They not only generate compact reduced macromodels, but also provide error bound for the reduced model. However, it is known that the standard TBR method [71] does not guarantee the passivity of the reduced system. This issue has been recently addressed in [72], [73]. In these work, passivity of the reduced model from the TBR procedure is ensured by obtaining the controllability and observability Grammians from the solution of two sets of Lur'e equations, which are dual of each other. The solution of Lur'e equations is obtained by using the Schur decomposition of the associated Hamiltonian matrices, which requires $203 n^{3}$ operations ( $n$ being the size of corresponding state-space system) [77]. This means that $406 n^{3}$ operations are required to obtain both the above discussed Grammians, leading to increased computational cost during the second-level reduction [77], [88].

In the next section we describe the proposed algorithm, which reduces the total cost of the second-level reduction using PR-TBR approach [72], [73], by half. This constitutes the second step of our proposed global model reduction strategy (GMOR). 


\subsection{Development of the Proposed Methodology for Generating Compact Macromodels}

This section presents the proposed methodology (referred to as fast positive-real TBR (FPTBR)) for generating compact macromodels of linear subnetworks obtained from first step of the proposed GMOR strategy. As such, this algorithm is the second stage of the GMOR technique. The details are provided below.

\subsubsection{Proposed Fast Passive Truncated Balanced Realization (FPTBR)}

The proposed algorithm is based on passive truncated balance realization (TBR). Unlike PR-TBR [73] type of algorithms (which requires solution of both the Lur'e equation and its dual, making the reduction process slower), the new algorithm computes the reduced passive model with computational cost equal to only the solution of one set of Lur'e equations, thereby enabling efficient computation of the reduced model.

The starting point of the second level of reduction is the reduced model (7.10) (7.12) obtained from the first-level reduction algorithm, which can be put in the state-space formulation as below.

$$
\begin{aligned}
& \dot{x}=A x+B u \\
& y=C x+D u
\end{aligned}
$$

In order to reduce the computational cost during second-level reduction using PR-TBR, we present appropriate application of results from control theory [92] to obtain the solution of ARE (3.24) and (3.26) at the cost of only one Schur decomposition of Hamiltonian matrix and solving one additional Sylvester equation. The Hamiltonian matrix corresponding to (3.24) can be written as 


$$
\boldsymbol{H}_{1}=\left[\begin{array}{cc}
\boldsymbol{A}-\boldsymbol{B} \boldsymbol{R}^{-1} \boldsymbol{C} & \boldsymbol{B} \boldsymbol{R}^{-1} \boldsymbol{B}^{T} \\
-\boldsymbol{C}^{T} \boldsymbol{R}^{-1} \boldsymbol{C} & -\left(\boldsymbol{A}-\boldsymbol{B} \boldsymbol{R}^{-1} \boldsymbol{C}\right)^{T}
\end{array}\right]
$$

Computing a reordered real schur decomposition, we can write $\boldsymbol{H}_{1}$ as

$$
\boldsymbol{H}_{1}=\boldsymbol{U} \boldsymbol{S}_{d} \boldsymbol{U}^{T} ; \quad \boldsymbol{S}_{\boldsymbol{d}}=\left[\begin{array}{ll}
\boldsymbol{S}_{-} & \boldsymbol{S} \\
0 & \boldsymbol{S}_{ \pm}
\end{array}\right]
$$

where $\boldsymbol{S}_{-}$contains all the stable while $\boldsymbol{S}_{+}$contains the unstable eigenvalues of $\boldsymbol{H}_{1}, \boldsymbol{S}$ is a matrix of order $n \times n$, and $\boldsymbol{U}$ is orthogonal. Partitioning $\boldsymbol{U}$ as

$$
\boldsymbol{U}=\left[\begin{array}{ll}
\boldsymbol{U}_{11} & \boldsymbol{U}_{12} \\
\boldsymbol{U}_{21} & \boldsymbol{U}_{22}
\end{array}\right]
$$

with each block $\boldsymbol{U}_{i j}$ being $n \times n$. Next the stable and unstable part of $\boldsymbol{S}_{\boldsymbol{d}}$ in (7.31) are separated by solving the Sylvester equation

$$
S_{-} Z-Z S_{+}+S=0
$$

Using (7.33) we can write (7.31) as

$$
\boldsymbol{H}_{1}=U\left[\begin{array}{ll}
\boldsymbol{I} & \boldsymbol{Z} \\
\mathbf{0} & \boldsymbol{I}
\end{array}\right]\left[\begin{array}{ll}
\boldsymbol{S}_{-} & \mathbf{0} \\
\mathbf{0} & \boldsymbol{S}_{ \pm}
\end{array}\right]\left[\begin{array}{ll}
\boldsymbol{I} & -\boldsymbol{Z} \\
\mathbf{0} & \boldsymbol{I}
\end{array}\right] \boldsymbol{U}^{T}
$$

or 


$$
\boldsymbol{H}_{1}=\left[\begin{array}{cc}
\boldsymbol{U}_{11} & \boldsymbol{U}_{11} \boldsymbol{Z}+\boldsymbol{U}_{12} \\
\boldsymbol{U}_{21} & \boldsymbol{U}_{21} \boldsymbol{Z}+\boldsymbol{U}_{22}
\end{array}\right]\left[\begin{array}{ll}
\boldsymbol{S}_{-} & \mathbf{0} \\
\mathbf{0} & \boldsymbol{S}_{+}
\end{array}\right]\left[\begin{array}{ll}
\boldsymbol{U}_{11} & \boldsymbol{U}_{11} \boldsymbol{Z}+\boldsymbol{U}_{12} \\
\boldsymbol{U}_{21} & \boldsymbol{U}_{21} \boldsymbol{Z}+\boldsymbol{U}_{22}
\end{array}\right]^{-1}
$$

It is evident from (7.35) that the stabilizing solution of ARE (3.24) is, $\boldsymbol{W}_{o}=\boldsymbol{U}_{21} \boldsymbol{U}_{11}^{-1}$ [88].

The Hamiltonian matrix corresponding to (3.26) can be written as

$$
\boldsymbol{H}_{2}=\left[\begin{array}{cc}
\boldsymbol{A}^{T}-\boldsymbol{C}^{T} \boldsymbol{R}^{-1} \boldsymbol{B}^{T} & \boldsymbol{C}^{T} \boldsymbol{R}^{-1} \boldsymbol{C} \\
-\boldsymbol{B} \boldsymbol{R}^{-1} \boldsymbol{B}^{T} & -\left(\boldsymbol{A}-\boldsymbol{B} \boldsymbol{R}^{-1} \boldsymbol{C}\right)
\end{array}\right]
$$

Next, defining

$$
J=\left[\begin{array}{ll}
0 & I \\
I & 0
\end{array}\right]
$$

it is evident that

$$
\boldsymbol{H}_{2}=-\boldsymbol{J H}_{1} \boldsymbol{J}
$$

Substituting $\boldsymbol{H}_{1}$ from (7.35)

$$
\boldsymbol{H}_{2}=\left[\begin{array}{ll}
\boldsymbol{0} & \boldsymbol{I} \\
\boldsymbol{I} & \boldsymbol{0}
\end{array}\right]\left[\begin{array}{ll}
\boldsymbol{U}_{11} & \boldsymbol{U}_{11} \boldsymbol{Z}+\boldsymbol{U}_{12} \\
\boldsymbol{U}_{21} & \boldsymbol{U}_{21} \boldsymbol{Z}+\boldsymbol{U}_{22}
\end{array}\right]\left[\begin{array}{cc}
-\boldsymbol{S}_{-} & \mathbf{0} \\
\boldsymbol{0} & -\boldsymbol{S}_{ \pm}
\end{array}\right]\left[\begin{array}{ll}
\boldsymbol{U}_{11} & \boldsymbol{U}_{11} \boldsymbol{Z}+\boldsymbol{U}_{12} \\
\boldsymbol{U}_{21} & \boldsymbol{U}_{21} \boldsymbol{Z}+\boldsymbol{U}_{22}
\end{array}\right]^{-1}\left[\begin{array}{ll}
\boldsymbol{0} & \boldsymbol{I} \\
\boldsymbol{I} & \mathbf{0}
\end{array}\right]
$$

or 


$$
\boldsymbol{H}_{2}=\left[\begin{array}{cc}
\boldsymbol{U}_{21} & \boldsymbol{U}_{21} \boldsymbol{Z}+\boldsymbol{U}_{22} \\
\boldsymbol{U}_{11} & \boldsymbol{U}_{11} \boldsymbol{Z}+\boldsymbol{U}_{11}
\end{array}\right]\left[\begin{array}{cc}
-\boldsymbol{S}_{-} & \mathbf{0} \\
\boldsymbol{0} & -\boldsymbol{S}_{+}
\end{array}\right]\left[\begin{array}{ll}
\boldsymbol{U}_{21} & \boldsymbol{U}_{21} \boldsymbol{Z}+\boldsymbol{U}_{22} \\
\boldsymbol{U}_{11} & \boldsymbol{U}_{11} \boldsymbol{Z}+\boldsymbol{U}_{12}
\end{array}\right]^{-1}
$$

(7.40)

From (7.40) it is evident that the stabilizing solution of ARE (3.26) is $\boldsymbol{W}_{c}=\left(\boldsymbol{U}_{11} \boldsymbol{Z}+\boldsymbol{U}_{12}\right)\left(\boldsymbol{U}_{21} \boldsymbol{Z}+\boldsymbol{U}_{22}\right)^{-1}$.

Hence, we are able to solve the dual Riccati equations ((3.24) and (3.26)), by using only one Schur decomposition (related computational cost: $203 n^{3}$ [77]) and solving an extra Sylvester equation (7.33) (related computational cost is $15 n^{3}$ [93]). Total number of operations in this case will be $218 n^{3}$ as against $406 n^{3}$ required to solve the dual Riccati equations separately. It is to be noted, that since $S_{-}$and $S_{+}$are in real schur form, the Sylvester equation can be efficiently solved using the Bartels-Stewart algorithm [83].

A brief summary of the proposed second-level passive reduction algorithm (FPTBR) for generating compact passive macromodels is given below, in terms of pseudocode. 
- Step 1: Obtain the state-space representation of the reduced system from (7.10) (7.12).

- Step 2: Perform the Schur decomposition of the corresponding Hamiltonian matrix as in (7.31). Use the Schur vectors spanning the stable invariant subspace to compute the observability matrix $\boldsymbol{W}_{o}=\boldsymbol{U}_{21} \boldsymbol{U}_{11}^{-1}$ using (7.35).

- Step 3: Solve the Sylvester equation (7.33) to obtain the matrix $Z$.

- Step 4: Use the matrix $Z$ and the Schur decomposition from Step 2 to write the Hamiltonian matrix corresponding to ARE (3.26) as in (7.40). Compute the controllability matrix $W_{c}$ by using the Schur vectors spanning the stable subspace in (7.40).

- Step 5: Use the above matrices to obtain the transformed system (3.21). Truncate the least controllable-observable states to obtain the further reduced system (3.22).

Fig. 7.17. Pseudocode for the proposed second-level reduction algorithm (FPTBR) for generating compact passive macromodels

\subsection{Numerical Results}

An example is presented in this section to demonstrate the validity and efficiency of the proposed FPTBR algorithm.

\subsubsection{Example: Four Port Network}

In this example, a four port network representing a typical signal path is considered (Fig. 7.18). It consists of two transmission line subnetworks with each subnetwork having two coupled lines represented by RLC lumped segments, and two via structures (represented by tabulated data). Each RLC coupled interconnect subnetwork is represented using 200 uniform lumped segments. 
Each via structure is modeled using [39], with passive state-space equations of order 110. The linear subnetwork with all sub-modules as described above is considered as the original network. The corresponding MNA is of order $2600 \times 2600$. The original system is analyzed using PRESS and a single reduced model of order 260 is obtained. This model is further reduced using the proposed FPTBR algorithm presented in Section 7.4.1, to a single compact macromodel of order 110. The time taken for this second-level reduction is 96 seconds. On the other hand, when second-level reduction is performed as outlined in PR-TBR algorithm [73], the time taken to produce the reduced model of same size (of order 110) is 181 seconds. Comparison of transient responses of original system with the reduced model obtained from FPTBR is given in Fig. 7.19.

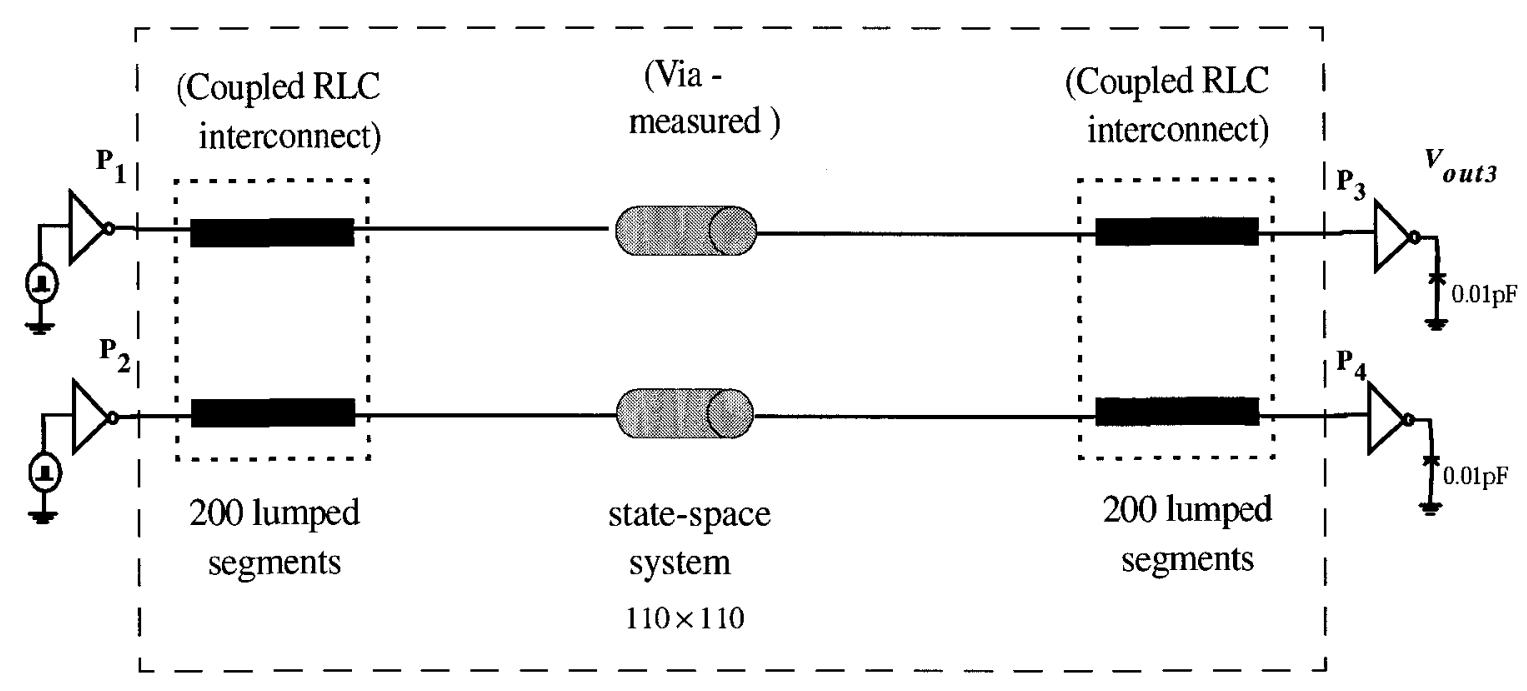

Fig. 7.18. Subnetwork consisting of diverse linear modules 


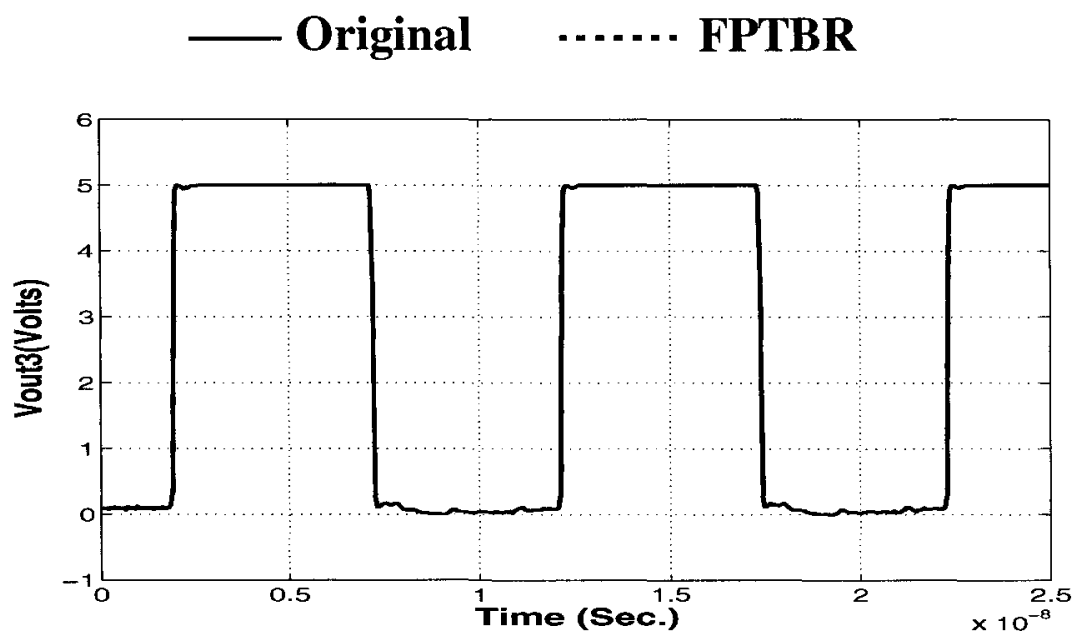

Fig. 7.19. Comparison of transient response

\subsection{Performance Comparison of the Proposed two-level GMOR Technique}

Recalling that a two-level global model reduction strategy was proposed in the beginning of this chapter. The first-level (PRESS, described in Section 7.2) involves the global reduction of the circuit consisting of diverse high-frequency elements. The second-level (FPTBR, described in Section 7.4) further reduces the model obtained from first-level reduction. In this section, we present some numerical results to show the validity and efficiency of the GMOR strategy. This is done by comparing the performance of GMOR with that of the conventional strategy (referred to as local model order reduction (LMOR)), where each module in the system is reduced individually and then they are concatenated to perform the overall analysis. 


\subsubsection{Example 1: Two Port Network}

In this example, a linear subnetwork (representing a typical signal path - Fig. 7.20) containing diverse modules, with two distributed transmission lines, one via (represented by tabulated data) and a RLC transmission line is considered. The distributed transmission lines were macromodeled in terms of passive state-space equations of order 128 using [14]. The via structure is modeled using [39], with passive state-space equations of order 166. The RLC interconnect is represented using 120 uniform lumped segments. The linear subnetwork with sub-modules as described above is considered as the original network. The original system is reduced using the GMOR (PRESS+FPTBR) strategy. For this, the original system is first reduced using the PRESS algorithm and a reduced model of order 150 is obtained. This is further reduced using the proposed second-level reduction algorithm (FPTBR) and a further compact macromodel of order 60 is obtained. Comparison of frequency and transient responses are given in Fig. 7.21 and Fig. 7.22, respectively. As seen they match accurately. Transient analysis of the original network required 124 seconds whereas the proposed macromodel required only 7 seconds.

Next, performance of the GMOR strategy is compared against the conventional approach (local model reduction (LMOR)). Here the RLC interconnect alone is first reduced using PRIMA and a reduced model of order 44 is obtained. Next, each of the sub-module is subjected to a passive second-level reduction. The orders of resulting four compact state-space systems for the same accuracy as that of GMOR, are as follows: RLC interconnect $(n=20)$, two distributed transmission lines $(n=96$, each), via $(n=132)$. Next, a transient simulation is performed using a concatenation of these four compact macromodels. The response in this case was 
identical to the one given in Fig. 7.22. However, this approach required 85 seconds. Table 7.3 summarizes the CPU times for this example.

Table. 7.3. Comparison of CPU time in sec. - example 1(Sun-Blade-100 machine)

\begin{tabular}{|c|c|c|}
\hline $\begin{array}{c}\text { Original } \\
\text { circuit }\end{array}$ & LMOR & GMOR \\
\hline 124 & 85 & 7 \\
\hline Speed-up & & 18 \\
\hline
\end{tabular}

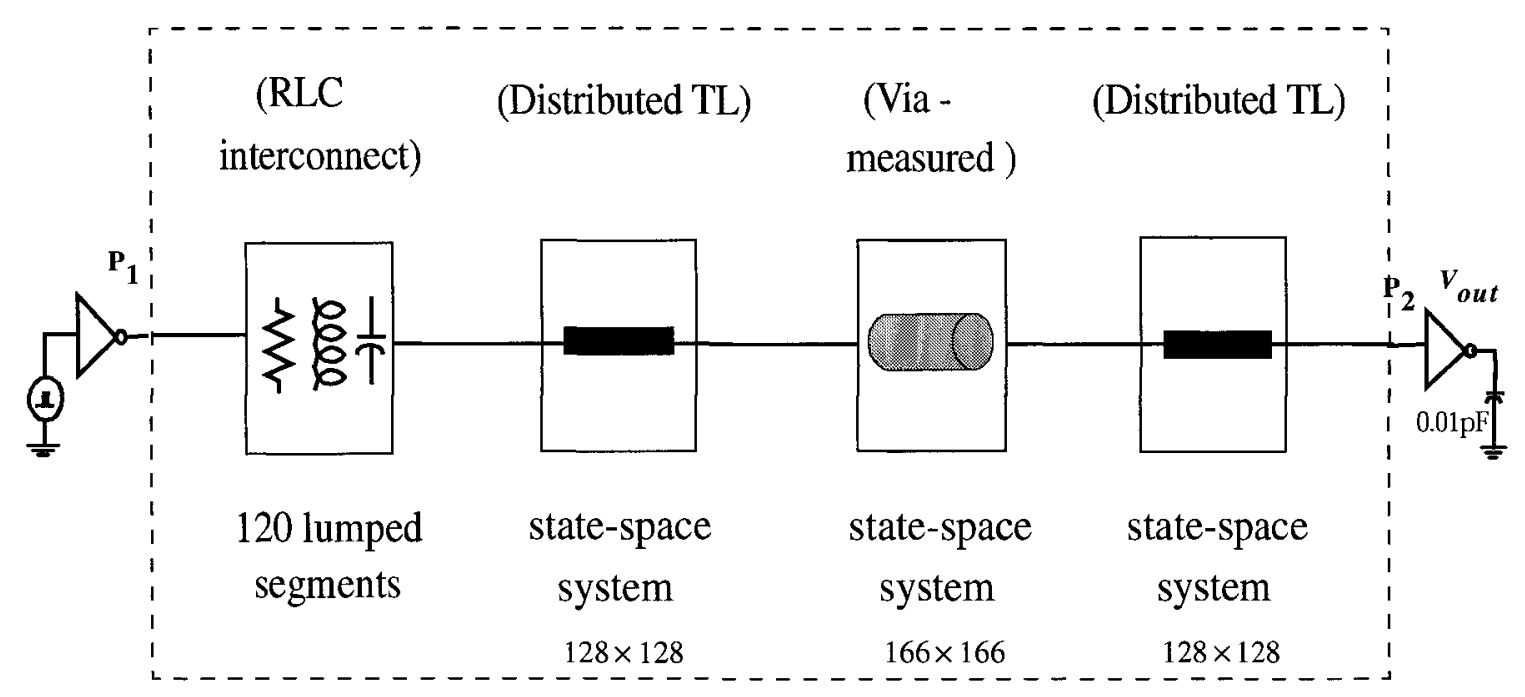

Fig. 7.20. Subnetwork consisting of diverse high-speed modules 
- Original - - GMOR
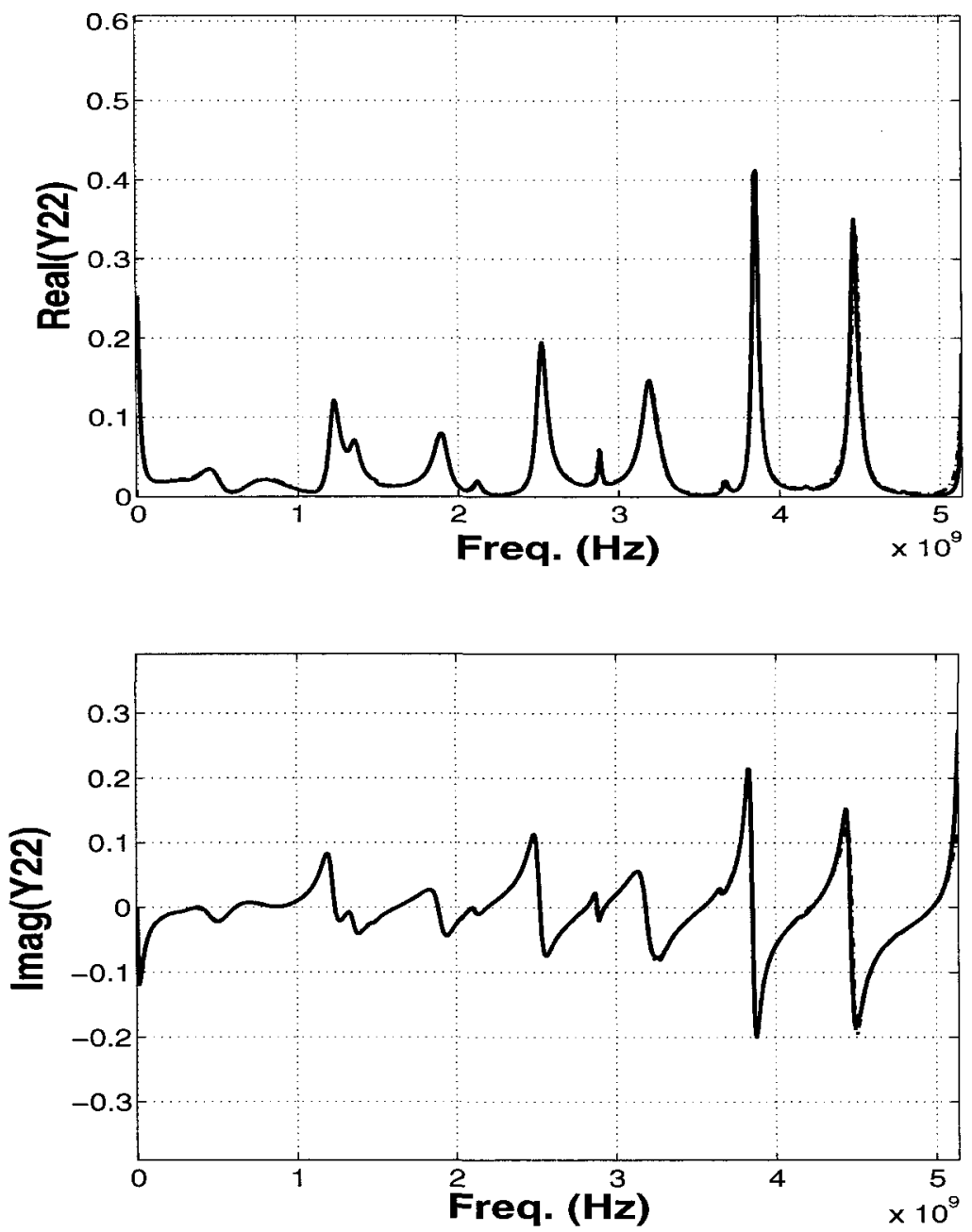

Fig. 7.21. Frequency responses - example 1 

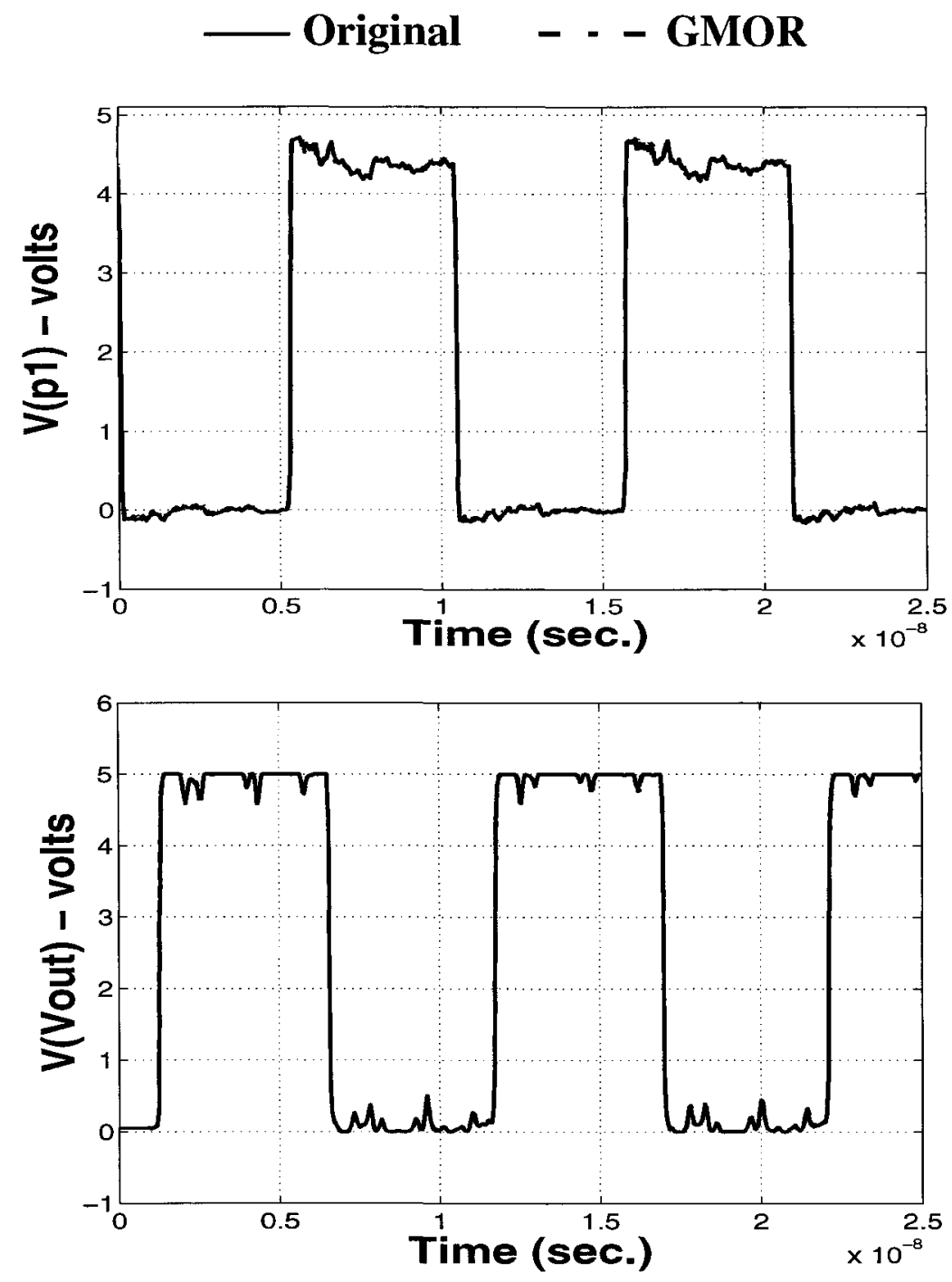

Fig. 7.22. Transient results - example 1

\subsubsection{Example 2: Four Port Network}

In this example, a four port network of Fig. 7.18 is considered. As mentioned in Section 7.5.1, each RLC coupled interconnect subnetwork is represented using 200 uniform lumped segments and each via structure is modeled with passive statespace equations of order 110 . The linear subnetwork with all sub-modules as 
described above is considered as the original network. The corresponding MNA is of order $2600 \times 2600$. Next, the subnetwork is analyzed using GMOR (PRESS+FPTBR) and a macromodel of order 110 is obtained. Transient analysis of the original network required 2400 seconds where as GMOR required only 90 seconds.

Performance of GMOR is next compared against the conventional approach (local model reduction (LMOR)). Here each RLC interconnect subnetwork is first reduced using PRIMA and a model of order 100 is obtained. Next, each of the sub-module is subjected to a passive second-level of reduction. The order of each coupled RLC interconnect subnetwork is 80 , while the order of each via structure is 86. Next, a transient simulation is performed using a concatenation of these compact macromodels. A comparison of the frequency responses of the original circuit, GMOR, and LMOR is shown in Fig. 7.23 and Fig. 7.24, while the transient responses are compared in Fig. 7.25. The transient analysis using LMOR approach required 350 seconds. Table 7.4 summarizes the CPU times for this example.

Table. 7.4. Comparison of CPU time in sec. - example 2 (Sun-Blade-100 machine)

\begin{tabular}{|c|c|c|}
\hline $\begin{array}{c}\text { Original } \\
\text { circuit }\end{array}$ & LMOR & GMOR \\
\hline 2400 & 350 & 90 \\
\hline Speed-up & & 27 \\
\hline
\end{tabular}


_ Original ..... GMOR -.-LMOR
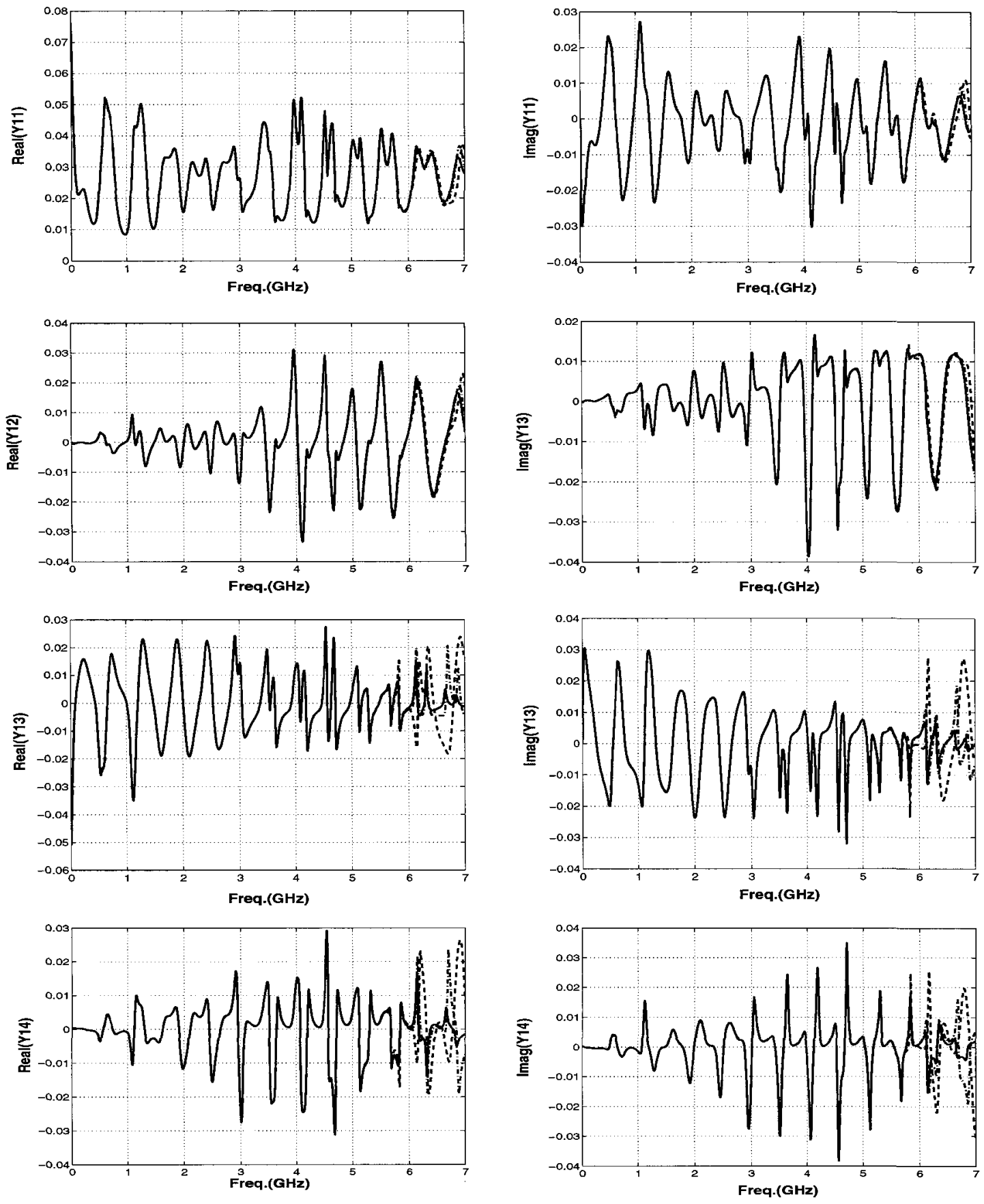

Fig. 7.23. Frequency responses - example 2 
_ Original $\ldots .$. GMOR $-\cdot$-LMOR
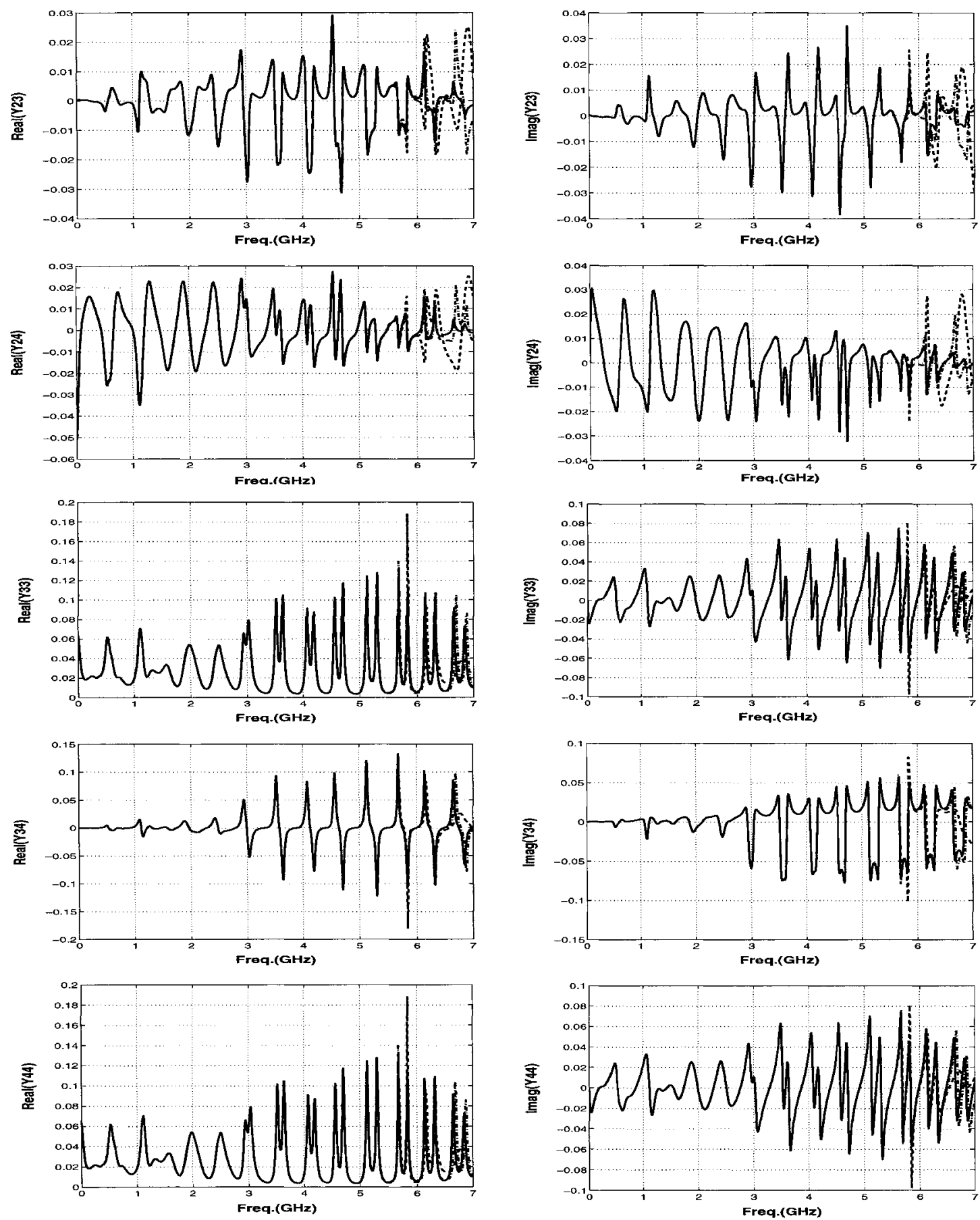

Fig. 7.24. Frequency responses - example 2 

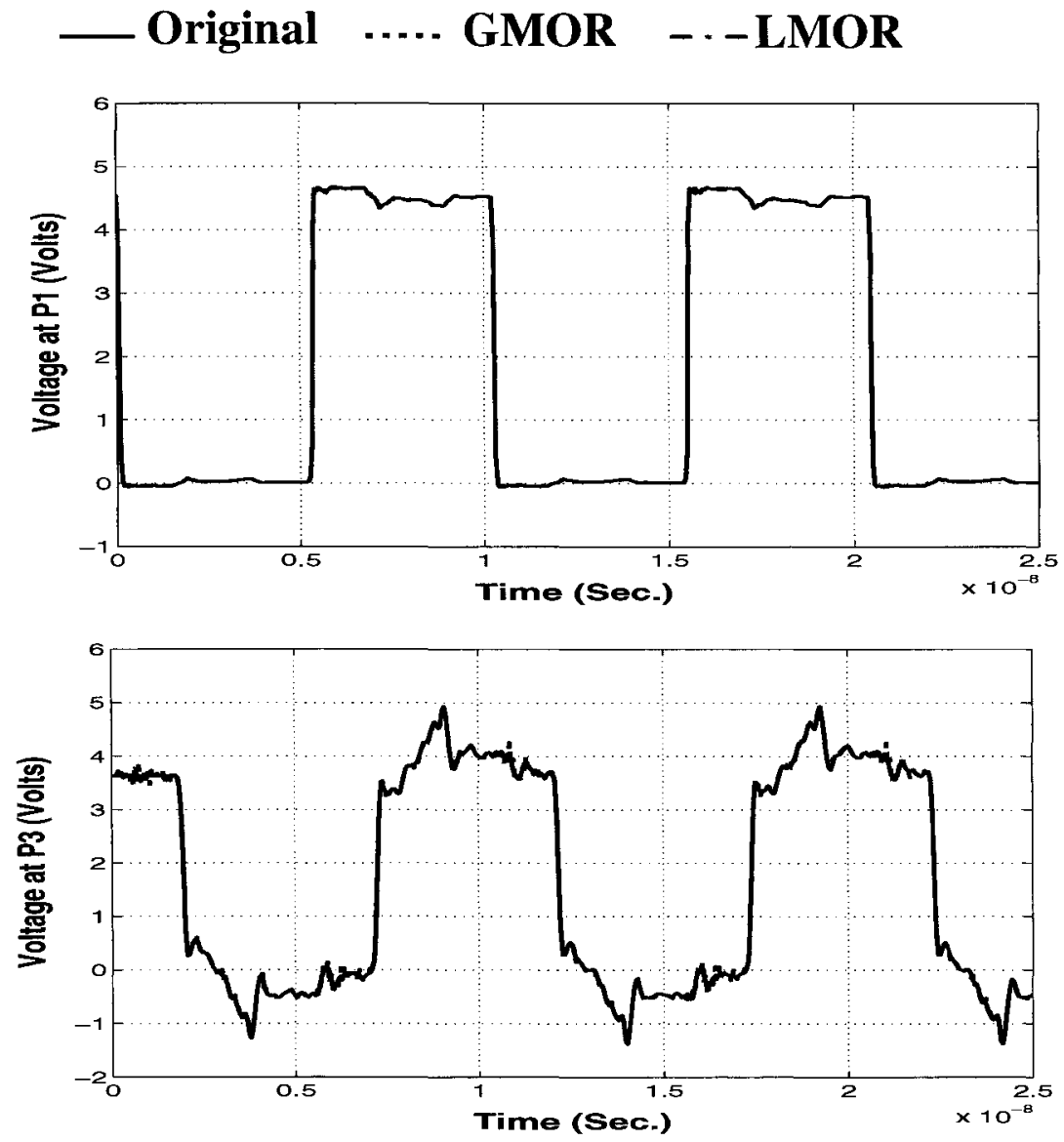

Fig. 7.25. Transient responses - example 2

\subsection{Conclusion}

In this chapter we have presented a two-step global model reduction (GMOR) algorithm. For the first step, a novel algorithm, PRESS has been presented, which provides a means to include the embedded systems described by passive statespace systems, in the Krylov subspace based passive reduction algorithm, such as PRIMA. In the second step, the proposed algorithm, FPTBR, performs a fast passive second-level reduction. The computational cost of this algorithm is 
approximately half, in comparison to the currently available algorithm for this purpose. In order to validate the usefulness of the proposed GMOR strategy, examples have been presented showing the comparative performance of the proposed two-level GMOR strategy with the conventional LMOR strategy. 


\section{CHAPTER 8}

\section{Conclusions and Future Work}

This chapter contains a summary of the work presented in this thesis. In addition, the possible directions for future work are discussed.

\subsection{Summary}

In this thesis, several new algorithms are presented to address the important, emerging issue of including tabulated interconnect networks in an overall circuit simulation. The presented algorithms can be broadly classified into two categories. The first category of algorithms address the issue of passive macromodeling of tabulated networks. The second category of algorithms provide a novel way of performing global passive model order reduction of modern signal paths containing multiple diverse high-speed modules.

\section{Passive Macromodeling of Tabulated Data:}

The algorithms under this category address various challenging issues such as, passivity verification, quantification of violation and enforcement, in passive macromodeling of tabulated networks. A summary of these algorithms is presented below. 
- A new, robust and practical algorithm is presented for obtaining passive macromodels of tabulated networks represented by small/medium sized models. This algorithm applies algebraic tests based on Hamiltonian matrices for passivity verification and quantification of violating regions. In addition, it compensates the nonpassive models using an efficient iterative approach based on perturbation. The presented algorithm is general in nature and can accept any form of tabulated data: $\mathrm{Y}, \mathrm{Z}$ or $\mathrm{S}$.

- For the tabulated data networks consisting of many ports and described over a wide range of frequency, the size of resulting models become very large. As a result, each iteration of the passivity enforcement process becomes computationally intensive. In view of this, a practical and efficient technique was presented in this thesis, which significantly reduces the time required for the passivity enforcement of large sized models. It intelligently combines the adaptive frequency sampling, reciprocal system formulation and the algebraic tests for passivity. As a result of this, the expensive computations during the passivity enforcement process are minimized.

- For the case of Y-parameter data, a global passivity enforcement technique with a guaranteed direction is presented. This approach has an advantage that during the passivity compensation process, no new passivity violating regions are introduced. As a result, the time taken for passivity enforcement is minimized.

\section{Compact Macromodeling of Signal Paths with Diverse Modules:}

The second category of algorithms presented in this thesis provide a novel way for performing global model reduction of modern signal paths. For this purpose, a two step global model-order reduction (GMOR) methodology has been presented. The details of these steps are summarised below. 
- In the first step, a novel algorithm (PRESS) has been presented, which enables the passive reduction of RLC interconnect circuits with embedded state-space systems. This work is the first of its kind for reducing the combined large RLC lumped circuits along with embedded state-space systems. The proposed algorithm uses the formulation which guarantees the passivity of the reduced model.

- The reduced order model from PRESS or other first-level reduction algorithms may not be optimal in size for performing transient analysis. An efficient algorithm (FPTBR) has been presented for the second-level reduction of interconnect models obtained from these first-level reduction algorithms. A major advantage of this algorithm is that, it reduces the cost of the second-level reduction scheme by half as compared to other available algorithms.

The algorithms presented in this thesis provide a unified strategy for the signal integrity analysis of high-speed interconnections. The major advantages of the proposed algorithms are as follows.

1) They can macromodel the tabulated networks of any size for wideband applications.

2) They are robust and practical and can be easily integrated in any industrial grade simulators.

3) They provide a novel means for performing a global passive reduction of signal paths consisting of diverse high-speed modules.

4) They provide a comprehensive solution for the system level signal integrity analysis. 


\section{$8.2 \quad$ Future Research}

The work presented in this thesis has laid the foundation for several future research projects. As a natural progression of the algorithms presented in thesis, given below is a list of some future work which can be undertaken.

1) Delay extraction from tabulated data:

The tabulated data of networks with long delays require a very high-order of rational approximation. This results in a large size of macromodels, leading to slow transient simulation. Further research is required to develop algorithms which can remove the inherent delay of the system before approximating the tabulated data.

2) Common pole-set for $S$-parameter data:

One of the open problem in approximating the scattering tabulated data is to ensure a common pole-set for all the elements of the scattering matrix. This problem needs to be addressed in the future work.

3) Reduction of computational cost for second-level reduction:

Although, the technique presented in this thesis reduces the computational cost of second-level reduction algorithms from $406 n^{3}$ to $218 n^{3}$, however even this may be higher for large systems. Hence, more work needs to be done to further reduce this cost. 


\section{APPENDIX - A Recursive Convolution}

In this section a brief description of the recursive convolution technique for transient analysis of pole-residue formulation of an interconnect network is given. Further details of this technique can be found in [19].

Once the partial fraction expansion of the transfer function is obtained, the relation between the input and output can be rewritten as

$$
B(s)=\underbrace{\left.c+\sum_{i=1}^{m^{\prime}} \frac{r_{i}}{s-p_{i}}\right)}_{Y(s)} A(s)
$$

where $A(s)$ and $B(s)$ are the Laplace-domain input and output signals respectively, while $Y(s)$ is the transfer function approximation in terms of poleresidue. The time-domain response is obtained by calculating the convolution integral given as

$$
b(t)=\int_{0}^{t} y(\tau) a(t-\tau) d \tau
$$

where $a(t), b(t)$ are the time-domain input and output signals respectively, while $y(t)$ is the time-domain representation of $Y(s)$. The convolution integral in (A.2) 
becomes progressively more expensive as the simulation time increases. Since transfer function $Y(s)$ is expressed as a sum of partial expansion (A.1), the time for the numerical convolution can be greatly reduced by taking advantage of recursive convolution.

In [19], recursive convolution and indirect numerical integration are used for fast transient simulation of transmission line networks. It is necessary to make assumptions about the nature of $a(t)$ that, its values at a discrete set of points suffice to specify $b(t)$ uniquely. As in the backward and forward Euler formulas, the excitation is assumed to be piecewise constant, i.e. $a(t)=$ constant, where $t_{n-1} \leq t \leq t_{n}$

By using the commutative property of convolution integral, (A.2) can be written as,

$$
b(t)=\int_{0}^{t} a(\tau) y(t-\tau) d \tau
$$

At time point $t_{n},(\mathrm{~A} .3)$ can be written as,

$$
b\left(t_{n}\right)=\int_{0}^{t_{n}} a(\tau) y\left(t_{n}-\tau\right) d \tau
$$

From (A.1), $y(t)$ can be written as

$$
y(t)=L^{-1}(Y(s))=L^{-1}\left(c+\sum_{i=1}^{m^{\prime}} \frac{r_{i}}{s+p_{i}}\right)=c \delta(t)+\sum_{i=1}^{m^{\prime}} r_{i} e^{-p_{i} t}
$$


where $L^{-1}$ is the inverse Laplace transform. Next, substituting (A.5) into (A.4) we get,

$$
b\left(t_{n}\right)=\int_{0}^{t_{n}} a(\tau)\left(c \delta\left(t_{n}-\tau\right)+\sum_{i=1}^{m^{\prime}} r_{i} e^{-p_{i}\left(t_{n}-\tau\right)}\right) d \tau
$$

or,

$$
b\left(t_{n}\right)=\int_{0}^{t_{n}} a(\tau) c \delta\left(t_{n}-\tau\right) d \tau+\sum_{i=10}^{m^{\prime}} \int_{0}^{t_{n}} a(\tau) r_{i} e^{-p_{i}\left(t_{n}-\tau\right)} d \tau
$$

Noticing that $\int_{0}^{t_{n}} a(\tau) c \delta\left(t_{n}-\tau\right) d \tau=c a\left(t_{n}\right)$, we can write (A.7) as,

$$
b\left(t_{n}\right)=c a\left(t_{n}\right)+\sum_{i=10}^{m^{\prime} t_{n}} a(\tau) r_{i} e^{-p_{i}\left(t_{n}-\tau\right)} d \tau
$$

Next, defining $\delta t_{n}=t_{n}-t_{n-1}$ and splitting the integral in (A.8) into two parts (one from 0 to $t_{n-1}$ and other from $t_{n-1}$ to $t_{n}$ ) we obtain,

$$
b\left(t_{n}\right)=c a\left(t_{n}\right)+\sum_{i=1}^{m^{\prime}}\left\{e^{-p_{i} \delta t_{n-1}^{t_{n-1}}} \int_{0} a(\tau) r_{i} e^{-p_{i}\left(t_{n-1}-\tau\right)} d \tau\right\}+\sum_{i=1 t_{n-1}}^{m^{\prime}} \int_{t_{n}}^{t_{n}} a(\tau) r_{i} e^{-p_{i}\left(t_{n}-\tau\right)} d \tau
$$

$$
t_{n-1}
$$

Now, substituting $\int_{0} a(\tau) r_{i} e^{-p_{i}\left(t_{n-1}-\tau\right)} d \tau=\tilde{b}_{i}\left(t_{n-1}\right)$ in (A.9) we get, 


$$
b\left(t_{n}\right)=c a\left(t_{n}\right)+\sum_{i=1}^{m^{\prime}} e^{-p_{i} \delta t_{n}} \tilde{b}_{i}\left(t_{n-1}\right)+\sum_{i=1 t_{n-1}}^{m^{\prime}} \int_{t_{n}}^{t_{n}} a(\tau) r_{i} e^{-p_{i}\left(t_{n}-\tau\right)} d \tau
$$

Next consider the integral term in (A.10). When $\delta t_{n}=t_{n}-t_{n-1}$ is very small $a(\tau)$ may be considered constant (i.e. $a(\tau)=a\left(t_{n-1}\right)$ ) within the interval $t_{n-1}$ to $t_{n}$. With this assumption (A.10) can be written as,

$$
b\left(t_{n}\right)=c a\left(t_{n}\right)+\sum_{i=1}^{m^{\prime}} e^{-p_{i} \delta t_{n}} \tilde{b}_{i}\left(t_{n-1}\right)+\sum_{i=1}^{m^{\prime}} r_{i} a\left(t_{n-1}\right) \int_{t_{n-1}}^{t_{n}} e^{-p_{i}\left(t_{n}-\tau\right)} d \tau
$$

Now defining a new variable

$$
m=t_{n}-\tau
$$

in (A.11), we get

$$
d \tau=-d m
$$

Substituting (A.12) and (A.13) in (A.11), we get

$$
b\left(t_{n}\right)=c a\left(t_{n}\right)+\sum_{i=1}^{m^{\prime}} e^{-p_{i} \delta t_{n}} \tilde{b}_{i}\left(t_{n-1}\right)+\sum_{i=1}^{m^{\prime}} r_{i} a\left(t_{n-1}\right) \int_{0}^{\delta t_{n}} e^{-p_{i} m} d m
$$

or,

$$
b\left(t_{n}\right)=c a\left(t_{n}\right)+\sum_{i=1}^{m^{\prime}} e^{-p_{i} \delta t_{n}} \tilde{b}_{i}\left(t_{n}-\delta t_{n}\right)+\sum_{i=1}^{m^{\prime}} \frac{r_{i} a\left(t_{n}-\delta t_{n}\right)}{p_{i}}\left(1-e^{-p_{i} \delta t_{n}}\right)
$$

Thus, 


$$
b\left(t_{n}\right)=c a\left(t_{n}\right)+\sum_{i=1}^{m^{\prime}} \tilde{b}_{i}\left(t_{n}\right)
$$

where,

$$
\tilde{b}_{i}\left(t_{n}\right)=\frac{r_{i}}{p_{i}}\left(1-e^{-p_{i} \delta t_{n}}\right) a\left(t_{n}-\delta t_{n}\right)+e^{-p_{i} \delta t_{n}} \tilde{b}_{i}\left(t_{n}-\delta t_{n}\right),
$$

and

$$
\delta t_{n}=t_{n}-t_{n-1}
$$

Using the recursive convolution formula (A.17), the convolution integral (A.2) is reduced into a linear operation that involves a simple update of parameters at each time point. 


\section{APPENDIX - B Passivity Enforcement via Perturbation of Hamiltonian Matrix}

In this section, a review of the passivity enforcement algorithm based on direct perturbation of the Hamiltonian matrix [43] is presented. This algorithm was being developed parallely at the same time as the proposed algorithm presented in this thesis, in chapter 4.

The tabulated frequency data is approximated by rational functions and converted into the state-space representation with the corresponding transfer function

$$
\boldsymbol{H}(s)=\boldsymbol{C}(s \boldsymbol{I}-\boldsymbol{A})^{-1} \boldsymbol{B}+\boldsymbol{D} .
$$

For the ease of presentation the scattering parameter formulation is considered in the rest of this section.

The passivity of the macromodel in (B.1) is checked based on the Theorem 1 and 2, Section 4.2 .2 of chapter 4 . Once the passivity violation regions are detected the passivity enforcement is carried out by directly perturbing the Hamiltonian matrix

$$
\boldsymbol{M}=\left[\begin{array}{cc}
\boldsymbol{A}+\boldsymbol{B}\left(\boldsymbol{I}-\boldsymbol{D}^{T} \boldsymbol{D}\right)^{-1} \boldsymbol{D}^{T} \boldsymbol{C} & \boldsymbol{B}\left(\boldsymbol{I}-\boldsymbol{D}^{T} \boldsymbol{D}\right)^{-1} \boldsymbol{B}^{T} \\
-\boldsymbol{C}^{T}\left(\boldsymbol{I}-\boldsymbol{D} \boldsymbol{D}^{T}\right)^{-1} \boldsymbol{C} & -\boldsymbol{A}^{T}-\boldsymbol{C}^{T} \boldsymbol{D}\left(\boldsymbol{I}-\boldsymbol{D}^{T} \boldsymbol{D}\right)^{-1} \boldsymbol{B}^{T}
\end{array}\right]
$$


The key idea of passivity compensation in this algorithm is depicted in Fig. B.1. Essentially the Hamitonian matrix $\boldsymbol{M}$ is perturbed by perturbing residue matrix $C$, so that the imaginary eigenvalue pairs are drawn closer in small increments till they bounce off the imaginary axis, in which case the model becomes passive. The error introduced in the response is tracked during the perturbation process and the algorithm quits in case the error introduced is more than the threshold.

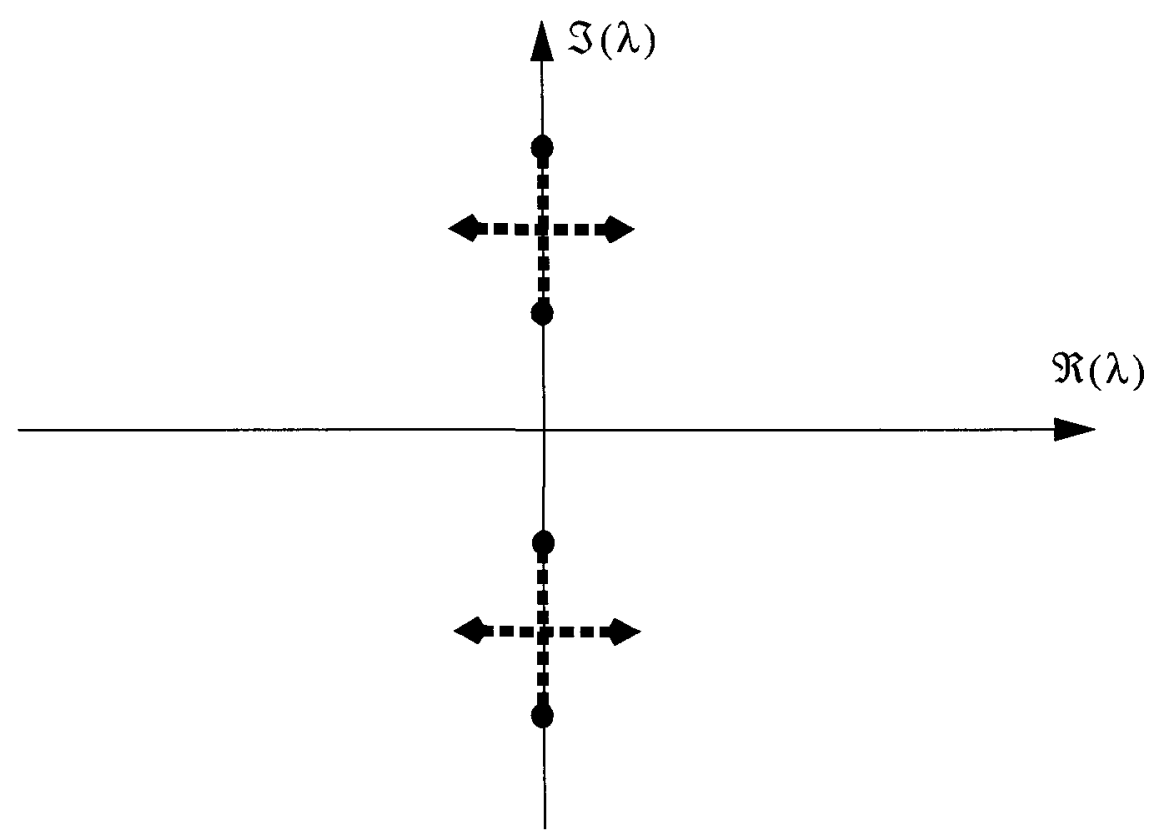

Fig. B.1. Perturbation of imaginary eigenvalues

Let $\Delta \boldsymbol{C}$ is the perturbation on the residue matrix $\boldsymbol{C}$ to move an imaginary eigenvalue $j \omega_{i}$ by $\Delta j \omega_{i}$ then by the first-order approximation

$$
j \Delta \omega_{i}=\frac{v_{i}^{H} J \Delta M v_{i}}{v_{i}^{H} J v_{i}}
$$


where

$$
\Delta M \approx\left[\begin{array}{cc}
-B \boldsymbol{R}_{1}^{-1} \boldsymbol{D}^{T} \Delta \boldsymbol{C} & 0 \\
\boldsymbol{C}^{T} \boldsymbol{S}_{1}^{-1} \Delta \boldsymbol{C}+\Delta \boldsymbol{C}^{T} \boldsymbol{S}_{1}^{-1} \boldsymbol{C} & \Delta \boldsymbol{C}^{T} \boldsymbol{D} \boldsymbol{R}_{1}^{-1} \boldsymbol{B}^{T}
\end{array}\right]
$$

$\boldsymbol{R}_{1}=\boldsymbol{D}^{T} \boldsymbol{D}-\boldsymbol{I}, \boldsymbol{S}_{1}=\boldsymbol{D} \boldsymbol{D}^{T}-\boldsymbol{I}$ and $\boldsymbol{v}_{i}$ is the right eigenvector of $\boldsymbol{M}$ corresponding to the imaginary eigenvalue $j \omega_{i}$. As evident here that the passivity enforcement iteration of this algorithm requires the knowledge of the imaginary eigenvalue/ eigenvector of the Hamiltonian matrix, whose size is twice the size of the order of the system. As against this the passivity enforcement algorithm presented in this thesis requires the eigenvalue/eigenvector (eq. 4.49) of the matrix whose size is that of the transfer function matrix, which is much smaller than the order of the system.

Through some mathematical manipulations, numerator of (B.3) can be expressed as

$$
\boldsymbol{v}_{i}^{H} \boldsymbol{J} \Delta \boldsymbol{M} \boldsymbol{v}_{i}=2 \operatorname{Real}\left\{\left(\boldsymbol{v}_{i 2}^{H} \boldsymbol{B} \boldsymbol{R}_{1}^{-1} \mathrm{D}^{T}+\boldsymbol{v}_{i 1}^{H} \boldsymbol{C}^{T} \boldsymbol{S}_{1}^{-1}\right) \Delta \boldsymbol{C} \boldsymbol{v}_{i 1}\right\}
$$

where, right eigenvector $v_{i}=\left[\begin{array}{l}v_{i 1} \\ v_{i 2}\end{array}\right]$. Using the property of Kronecker product [87], (B.5) can be rewritten as

$$
\boldsymbol{v}_{i}^{H} J \Delta M_{1} v_{i}=2 \operatorname{Real}\left\{\boldsymbol{v}_{i 1}^{T} \otimes\left(v_{i 2}^{H} B R_{1}^{-1} D^{T}+v_{i 1}^{H} C^{T} S_{1}^{-1}\right)\right\} \operatorname{vec}(\Delta C)
$$


Using (B.6), the required perturbation $\operatorname{vec}(\Delta C)$ can be calculated by solving the following equation at the frequency points where the singular value of $\boldsymbol{H}(s)$ is equal to one.

$$
2 \operatorname{Re}\left\{\boldsymbol{v}_{i 1}^{T} \otimes\left(v_{i 2}^{H} B R_{1}^{-1} D^{T}+v_{i 1}^{H} C^{T} S_{1}^{-1}\right)\right\} \operatorname{vec}(\Delta C)=-\operatorname{Im}\left\{\boldsymbol{v}_{i}^{H} J v_{i}\right\} \Delta \omega_{i}
$$

or

$$
N X=b
$$

where each row of matrix $N$ corresponds to the LHS of (B.7) corresponding to the imaginary eigenvalue of $\boldsymbol{M}$, while $\boldsymbol{b}$ stores the corresponding RHS of (B.7). With the perturbation $\Delta C$, the $L_{2}$ norm of the error in the response if given by [81]

$$
\|\Delta \boldsymbol{H}\|_{2}^{2}=\operatorname{trace}\left(\Delta \boldsymbol{C P} \Delta \boldsymbol{C}^{T}\right)
$$

where, $\boldsymbol{P}$ is the controllability Grammian obtained as the solution of the Lyapunov equation

$$
\boldsymbol{A P}+\boldsymbol{P A}{ }^{T}+\boldsymbol{B} \boldsymbol{B}^{T}=\mathbf{0}
$$

The macromodel is compensated for passivity by perturbing the imaginary eigenvalues of the Hamiltonian matrix $\boldsymbol{M}$, according to (B.8) subjected to minimizing (B.9), in an iterative manner. The iterations are continued till all the imaginary eigenvalues are exhausted. 


\section{APPENDIX - C Proof of Positive-Real Theorem}

In this section, the proof of theorem 2, introduced in Section 6.2 of chapter 6, and its corollary is presented. The proofs are on the similar lines as that of theorem 2 presented in Section 4.2.2 of chapter 4 for scattering parameters.

Theorem 2: The real part of the symmetric admittance matrix, $\boldsymbol{F}\left(j \omega_{0}\right)$, is singular if $j \omega_{0}$ is an eigenvalue of the corresponding Hamiltonian matrix $\boldsymbol{M}$, provided $\boldsymbol{D}+\boldsymbol{D}^{T}$ is a positive definite matrix.

where,

$$
\boldsymbol{M}=\left[\begin{array}{cc}
\boldsymbol{A}-\boldsymbol{B}\left(\boldsymbol{D}+\boldsymbol{D}^{T}\right)^{-1} \boldsymbol{C} & \boldsymbol{B}\left(\boldsymbol{D}+\boldsymbol{D}^{T}\right)^{-1} \boldsymbol{B}^{T} \\
-C^{T}\left(\boldsymbol{D}+\boldsymbol{D}^{T}\right)^{-1} \boldsymbol{C} & -\boldsymbol{A}^{T}+\boldsymbol{C}^{T}\left(\boldsymbol{D}+\boldsymbol{D}^{T}\right)^{-1} \boldsymbol{B}^{T}
\end{array}\right]
$$

Proof of Theorem 2: In order to relate the imaginary eigenvalues of Hamiltonian matrix $\boldsymbol{M}$ with the locations where $\boldsymbol{F}\left(j \omega_{0}\right)$, is singular, let us consider that $j \omega_{0}$ is an imaginary eigenvalue of $\boldsymbol{M}$, i.e.

$$
\boldsymbol{M}\left[\begin{array}{l}
r \\
s
\end{array}\right]=j \omega_{0}\left[\begin{array}{l}
r \\
s
\end{array}\right]
$$


where, $\left[\begin{array}{ll}r^{T} & \boldsymbol{s}^{T}\end{array}\right]^{T}$ is the eigenvector of $\boldsymbol{M}$ corresponding to the eigenvalue $j \omega_{0}$. Using (C.1) and (C.2), we get

$$
\left[\begin{array}{cc}
\boldsymbol{A}-\boldsymbol{B}\left(\boldsymbol{D}+\boldsymbol{D}^{T}\right)^{-1} \boldsymbol{C} & \boldsymbol{B}\left(\boldsymbol{D}+\boldsymbol{D}^{T}\right)^{-1} \boldsymbol{B}^{T} \\
-\boldsymbol{C}^{T}\left(\boldsymbol{D}+\boldsymbol{D}^{T}\right)^{-1} \boldsymbol{C} & -\boldsymbol{A}^{T}+\boldsymbol{C}^{T}\left(\boldsymbol{D}+\boldsymbol{D}^{T}\right)^{-1} \boldsymbol{B}^{T}
\end{array}\right]\left[\begin{array}{l}
\boldsymbol{r} \\
s
\end{array}\right]=j \omega_{0}\left[\begin{array}{l}
\boldsymbol{r} \\
s
\end{array}\right]
$$

Notice in (C.3) that, $\left(\boldsymbol{D}+\boldsymbol{D}^{T}\right)^{-1}$ exists, as it is assumed that $\left(\boldsymbol{D}+\boldsymbol{D}^{T}\right)$ is positive definite. Re-arranging (C.3), we have

$$
\left[\begin{array}{ll}
-\boldsymbol{B}\left(\boldsymbol{D}+\boldsymbol{D}^{T}\right)^{-1} \boldsymbol{C} & \boldsymbol{B}\left(\boldsymbol{D}+\boldsymbol{D}^{T}\right)^{-1} \boldsymbol{B}^{T} \\
-\boldsymbol{C}^{T}\left(\boldsymbol{D}+\boldsymbol{D}^{T}\right)^{-1} \boldsymbol{C} & \boldsymbol{C}^{T}\left(\boldsymbol{D}+\boldsymbol{D}^{T}\right)^{-1} \boldsymbol{B}^{T}
\end{array}\right]\left[\begin{array}{l}
\boldsymbol{r} \\
\boldsymbol{s}
\end{array}\right]=j \omega_{0}\left[\begin{array}{l}
\boldsymbol{r} \\
s
\end{array}\right]+\left[\begin{array}{ll}
-\boldsymbol{A} & \\
\boldsymbol{A}^{T}
\end{array}\right]\left[\begin{array}{l}
\boldsymbol{r} \\
s
\end{array}\right]
$$

After simple mathematical manipulations (C.4) can be written as

$$
\left[\begin{array}{c}
\boldsymbol{B} \\
\boldsymbol{C}^{T}
\end{array}\right]\left(\boldsymbol{D}+\boldsymbol{D}^{T}\right)^{-1}\left[\begin{array}{ll}
-\boldsymbol{C} & \boldsymbol{B}^{T}
\end{array}\right]\left[\begin{array}{l}
\boldsymbol{r} \\
\boldsymbol{s}
\end{array}\right]=\left[\begin{array}{ll}
\left(j \omega_{0} \boldsymbol{I}-\boldsymbol{A}\right) & \\
& \left(j \omega_{0} \boldsymbol{I}+\boldsymbol{A}^{T}\right)
\end{array}\right]\left[\begin{array}{l}
\boldsymbol{r} \\
\boldsymbol{s}
\end{array}\right]
$$

which can re-written as

$$
\left[\begin{array}{c}
\boldsymbol{B} \\
\boldsymbol{C}^{T}
\end{array}\right] \boldsymbol{u}=\left[\begin{array}{cc}
\left(j \omega_{0} \boldsymbol{I}-\boldsymbol{A}\right) & \\
& \left(j \omega_{0} \boldsymbol{I}+\boldsymbol{A}^{T}\right)
\end{array}\right]\left[\begin{array}{l}
\boldsymbol{r} \\
\boldsymbol{s}
\end{array}\right]
$$

or 


$$
\left[\begin{array}{l}
\boldsymbol{r} \\
\boldsymbol{s}
\end{array}\right]=\left[\begin{array}{ll}
\left(j \omega_{0} \boldsymbol{I}-\boldsymbol{A}\right) & \\
& \left(j \omega_{0} \boldsymbol{I}+\boldsymbol{A}^{T}\right)
\end{array}\right]^{-1}\left[\begin{array}{l}
\boldsymbol{B} \\
\boldsymbol{C}^{T}
\end{array}\right] \boldsymbol{u}
$$

where

$$
\boldsymbol{u}=\left(\boldsymbol{D}+\boldsymbol{D}^{T}\right)^{-1}\left[\begin{array}{ll}
-\boldsymbol{C} & \boldsymbol{B}^{T}
\end{array}\right]\left[\begin{array}{l}
\boldsymbol{r} \\
\boldsymbol{s}
\end{array}\right]
$$

Using (C.7) and (C.8), we get

$$
\left(\boldsymbol{D}+\boldsymbol{D}^{T}\right) \boldsymbol{u}=\left[\begin{array}{ll}
-\boldsymbol{C} & \boldsymbol{B}^{T}
\end{array}\right]\left[\begin{array}{ll}
\left(j \omega_{0} \boldsymbol{I}-\boldsymbol{A}\right)^{-1} & \left(j \omega_{0} \boldsymbol{I}+\boldsymbol{A}^{T}\right)^{-1}
\end{array}\right]\left[\begin{array}{c}
\boldsymbol{B} \\
\boldsymbol{C}^{T}
\end{array}\right] \boldsymbol{u}
$$

Equation (C.9) can be re-arranged as

$$
\left(\boldsymbol{D}+\boldsymbol{D}^{T}\right) \boldsymbol{u}=\left[\begin{array}{ll}
-\boldsymbol{C} & \boldsymbol{B}^{T}
\end{array}\right]\left[\begin{array}{c}
\left(j \omega_{0} \boldsymbol{I}-\boldsymbol{A}\right)^{-1} \boldsymbol{B} \\
\left(j \omega_{0} \boldsymbol{I}+\boldsymbol{A}^{T}\right)^{-1} \boldsymbol{C}^{T}
\end{array}\right] \boldsymbol{u}
$$

or

$$
\left(\boldsymbol{C}\left(j \omega_{0} \boldsymbol{I}-\boldsymbol{A}\right)^{-1} \boldsymbol{B}+\boldsymbol{D}+\boldsymbol{B}^{T}\left(-j \omega_{0} \boldsymbol{I}-\boldsymbol{A}^{T}\right)^{-1} \boldsymbol{C}^{T}+\boldsymbol{D}^{T}\right) \boldsymbol{u}=\mathbf{0}
$$

Noting that $\boldsymbol{F}\left(j \omega_{0}\right)=\left(\boldsymbol{C}\left(j \omega_{0} \boldsymbol{I}-\boldsymbol{A}\right)^{-1} \boldsymbol{B}+\boldsymbol{D}+\boldsymbol{B}^{T}\left(-j \omega_{0} \boldsymbol{I}-\boldsymbol{A}^{T}\right)^{-1} \boldsymbol{C}^{T}+\boldsymbol{D}^{T}\right) / 2$, we can write (C.11) as

$$
\boldsymbol{F}\left(j \omega_{0}\right) \boldsymbol{u}=\mathbf{0}
$$

It is evident from (C.11) that $\boldsymbol{F}(j \omega)$ is singular at $j \omega_{0}$. Next, correlating this information with the initial assumption we started with, i.e., $j \omega_{0}$ is the eigenvalue 
of the Hamiltonian matrix $\boldsymbol{M}$, we can infer that an imaginary eigenvalue of Hamiltonian matrix $\boldsymbol{M}$ corresponds to the frequency at which $\boldsymbol{F}(j \omega)$ is singular.

Using the similar approach, corollary of the theorem 2 can be proved as follows.

Corollary of Theorem 2: $j \omega_{0}$ is an imaginary eigenvalue of $\boldsymbol{M}$ if, the real part of the corresponding symmetric admittance matrix, $\boldsymbol{F}\left(j \omega_{0}\right)$ is singular, provided $\boldsymbol{D}+\boldsymbol{D}^{T}$ is a positive definite matrix".

Proof of Corollary of Theorem 2: Let us start by assuming that $\boldsymbol{F}(j \omega)$ is singular at frequency $\omega_{0}$. This means that $\boldsymbol{F}\left(j \omega_{0}\right)$ has an eigenvalue which is zero, i.e.

$$
\boldsymbol{F}\left(j \omega_{0}\right) \boldsymbol{u}=\mathbf{0}
$$

where, $\boldsymbol{u}$ is the eigenvector in the null space of $\boldsymbol{F}\left(j \omega_{0}\right)$. Noting that $\boldsymbol{F}\left(j \omega_{0}\right)=\left(\boldsymbol{C}\left(j \omega_{0} \boldsymbol{I}-\boldsymbol{A}\right)^{-1} \boldsymbol{B}+\boldsymbol{D}+\boldsymbol{B}^{T}\left(-j \omega_{0} \boldsymbol{I}-\boldsymbol{A}^{T}\right)^{-1} \boldsymbol{C}^{T}+\boldsymbol{D}^{T}\right) / 2, \quad$ (C.13) can be rewritten as

$$
\left(\boldsymbol{C}\left(j \omega_{0} \boldsymbol{I}-\boldsymbol{A}\right)^{-1} \boldsymbol{B}+\boldsymbol{D}+\boldsymbol{B}^{T}\left(-j \omega_{0} \boldsymbol{I}-\boldsymbol{A}^{T}\right)^{-1} \boldsymbol{C}^{T}+\boldsymbol{D}^{T}\right) \boldsymbol{u}=\mathbf{0}
$$

or

$$
\boldsymbol{C}\left(j \omega_{0} \boldsymbol{I}-\boldsymbol{A}\right)^{-1} \boldsymbol{B} \boldsymbol{u}+\boldsymbol{B}^{T}\left(-j \omega_{0} \boldsymbol{I}-\boldsymbol{A}^{T}\right)^{-1} \boldsymbol{C}^{T} \boldsymbol{u}=-\left(\boldsymbol{D}+\boldsymbol{D}^{T}\right) \boldsymbol{u}
$$

Substituting

$$
-\left(j \omega_{0} \boldsymbol{I}-\boldsymbol{A}\right)^{-1} \boldsymbol{B} \boldsymbol{u}=\boldsymbol{r}
$$

and 


$$
\left(-j \omega_{0} I-A^{T}\right)^{-1} C^{T} \boldsymbol{u}=s
$$

(C.15) can be written as

$$
-\boldsymbol{C r}+\boldsymbol{B}^{T} \boldsymbol{s}=-\left(\boldsymbol{D}+\boldsymbol{D}^{T}\right) \boldsymbol{u}
$$

or

$$
\left[\begin{array}{ll}
-\boldsymbol{C} & \boldsymbol{B}^{T}
\end{array}\right]\left[\begin{array}{l}
\boldsymbol{r} \\
\boldsymbol{s}
\end{array}\right]=-\left(\boldsymbol{D}+\boldsymbol{D}^{T}\right) \boldsymbol{u}
$$

or

$$
-\left(\boldsymbol{D}+\boldsymbol{D}^{T}\right)^{-1}\left[\begin{array}{ll}
\boldsymbol{C} & \boldsymbol{B}^{T}
\end{array}\right]\left[\begin{array}{l}
\boldsymbol{r} \\
\boldsymbol{s}
\end{array}\right]=\boldsymbol{u}
$$

Next, using (C.16) and (C.17) we get

$$
-\boldsymbol{B} \boldsymbol{u}=\left(j \omega_{0} \boldsymbol{I}-\boldsymbol{A}\right) \boldsymbol{r}
$$

and

$$
-\boldsymbol{C}^{T} \boldsymbol{u}=\left(j \omega_{0} \boldsymbol{I}+\boldsymbol{A}^{T}\right) \boldsymbol{s}
$$

respectively. Combining (C.21) and (C.22) we get

$$
\left[\begin{array}{c}
-\boldsymbol{B} \\
-\boldsymbol{C}^{T}
\end{array}\right] \boldsymbol{u}=\left[\begin{array}{cc}
\left(j \omega_{0} \boldsymbol{I}-\boldsymbol{A}\right) & 0 \\
0 & \left(j \omega_{0} \boldsymbol{I}+\boldsymbol{A}^{T}\right)
\end{array}\right]\left[\begin{array}{l}
\boldsymbol{r} \\
\boldsymbol{s}
\end{array}\right]
$$

Now, substituting $\boldsymbol{u}$ from (C.20) in (C.23) we get 


$$
\left[\begin{array}{c}
\boldsymbol{B} \\
\boldsymbol{C}^{T}
\end{array}\right]\left(\boldsymbol{D}+\boldsymbol{D}^{T}\right)^{-1}\left[\begin{array}{ll}
-\boldsymbol{C} & \boldsymbol{B}
\end{array}\right]\left[\begin{array}{l}
\boldsymbol{r} \\
\boldsymbol{s}
\end{array}\right]=\left[\begin{array}{cc}
\left(j \omega_{0} \boldsymbol{I}-\boldsymbol{A}\right) & 0 \\
0 & \left(j \omega_{0} \boldsymbol{I}+\boldsymbol{A}^{T}\right)
\end{array}\right]\left[\begin{array}{l}
\boldsymbol{r} \\
\boldsymbol{s}
\end{array}\right]
$$

or

$$
\left[\begin{array}{cc}
-\boldsymbol{B}\left(\boldsymbol{D}+\boldsymbol{D}^{T}\right)^{-1} \boldsymbol{C} & \boldsymbol{B}\left(\boldsymbol{D}+\boldsymbol{D}^{T}\right)^{-1} \boldsymbol{B}^{T} \\
-\boldsymbol{C}^{T}\left(\boldsymbol{D}+\boldsymbol{D}^{T}\right)^{-1} \boldsymbol{C} & \boldsymbol{C}^{T}\left(\boldsymbol{D}+\boldsymbol{D}^{T}\right)^{-1} \boldsymbol{B}^{T}
\end{array}\right]\left[\begin{array}{l}
\boldsymbol{r} \\
\boldsymbol{s}
\end{array}\right]=j \omega_{0}\left[\begin{array}{l}
\boldsymbol{r} \\
\boldsymbol{s}
\end{array}\right]+\left[\begin{array}{cc}
\boldsymbol{A} & 0 \\
0 & \boldsymbol{A}^{T}
\end{array}\right]\left[\begin{array}{l}
\boldsymbol{r} \\
\boldsymbol{s}
\end{array}\right]
$$

or

$$
\left[\begin{array}{cc}
\boldsymbol{A}-\boldsymbol{B}\left(\boldsymbol{D}+\boldsymbol{D}^{T}\right)^{-1} \boldsymbol{C} & \boldsymbol{B}\left(\boldsymbol{D}+\boldsymbol{D}^{T}\right)^{-1} \boldsymbol{B}^{T} \\
-\boldsymbol{C}^{T}\left(\boldsymbol{D}+\boldsymbol{D}^{T}\right)^{-1} \boldsymbol{C} & -\boldsymbol{A}^{T}+\boldsymbol{C}^{T}\left(\boldsymbol{D}+\boldsymbol{D}^{T}\right)^{-1} \boldsymbol{B}^{T}
\end{array}\right]\left[\begin{array}{l}
\boldsymbol{r} \\
\boldsymbol{s}
\end{array}\right]=j \omega_{0}\left[\begin{array}{l}
\boldsymbol{r} \\
\boldsymbol{s}
\end{array}\right]
$$

Noting that the Hamiltonian matrix $\boldsymbol{M}$ is defined as in (C.1), we can write (C.26) as

$$
\boldsymbol{M}\left[\begin{array}{l}
r \\
s
\end{array}\right]=j \omega_{0}\left[\begin{array}{l}
r \\
s
\end{array}\right]
$$

It is evident from (C.27) that $j \omega_{0}$ is the eigenvalue of Hamiltonian matrix $\boldsymbol{M}$ as defined in (C.1). Next correlating this information with the initial assumption we started with, i.e. $\boldsymbol{F}(j \omega)$ is singular at frequency $\omega_{0}$, we can infer that the frequency point at which $\boldsymbol{F}(j \omega)$ is singular, corresponds to the imaginary eigenvalue of the Hamiltonian matrix $\boldsymbol{M}$. 


\section{References}

[1] H. B. Bakoglu, Circuits, Interconnections and packaging for VLSI. Addison-Wesley, Reading MA, 1990.

[2] A. Deustsch, "Electrical characteristics of interconnections for high-performance systems," Proceedings of the IEEE, vol. 86, no. 2, pp. 315-355, Feb. 1998.

[3] M. Nakhla and R. Achar, Multimedia Book Series on Signal Integrity. OMNIZ Global Knowledge Corporation (www.omniz.com), ON: 2002.

[4] C. Paul, Analysis of Multiconductor Transmission Lines. NY: John Wiley and Sons Inc., 1994.

[5] R. Achar and M. Nakhla, "Simulation of high-speed interconnects," Proceedings of The IEEE, vol. 89, pp. 693-728, May 2001.

[6] L. T. Pillage and R. A. Rohrer, "Asymptotic waveform evaluation for timing analysis," IEEE Trans. Computer-Aided Design, vol. 9, pp. 352-366, Apr. 1990.

[7] E. Chiprout and M. Nakhla, Asymptotic Waveform Evaluation and Moment Matching for Interconnect Analysis. Boston: Kluwer Academic Publishers, 1993.

[8] E. Chiprout and M. Nakhla, "Analysis of interconnect networks using complex frequency hopping," IEEE Trans. Computer-Aided Design, pp. 186-199, Feb. 1995.

[9] P. Feldmann and R. W. Freund, "Efficient linear circuit analysis by Padé approximation via the Lanczos process," IEEE Trans. Computer-Aided Design, vol. 14, pp. 639-649, May 1995.

[10] K. J. Kerns, I. L. Wemple, and A. T. Yang, "Preservation of passivity during RLC network reduction via split congruence transformations," Proc. 34th ACM/IEEE Design Automation Conf., Anaheim, CA, June 1997, pp. 34-39.

[11] M. Celik and A. C. Cangellaris, "Simulation of multiconductor transmission lines using Krylov subspace order-reduction techniques," IEEE Trans. Computer-Aided Design, vol. 16, pp. 485-496, May 1997.

[12] M. Silveria, M. Kamon, I. Elfadel and J. White, “A coordinate-transformed Arnoldi 
algorithm for generating guaranteed stable reduced-order models of arbitrary RLC circuits", in Proc. IEEE ICCAD, Nov. 96.

[13] A. Odabasioglu, M. Celik, and L. T. Pileggi, "PRIMA: Passive reduced-order interconnect macromodeling algorithm," IEEE Trans. Computer-Aided Design, vol. 17, pp. 645-654, Aug. 1998.

[14] Q. Yu, J. M. L. Wang and E. S. Kuh, "Passive multipoint moment-matching model order reduction algorithm on multiport distributed interconnect networks," IEEE Trans on. Circuits and Systems - I, pp. 140-160, Jan. 99.

[15] A. Dounavis, E. Gad, R. achar and M. Nakhla, "Passive model-reduction of multiport distributed networks including frequency-dependent parameters," IEEE Trans. on Microwave Theory and Techniques, pp. 2325-2334, Dec. 2000.

[16] J. E. Schutt-Aine and R. Mittra, "Scattering parameter transient analysis of transmission lines loaded with nonlinear terminations," IEEE Trans. on Microwave Theory and Techniques, vol. 36, pp. 529-536, 1988.

[17] A. R. Djordjevic and T. K. Sarkar, "Analysis of lossy transmission lines with arbitrary nonlinear terminal networks", IEEE Trans. on Microwave Theory and Techniques, vol. 34, no. 6, pp. 660-666, June 1986.

[18] D. Winklestein, M. B. Steer, and R. Pomeerleau, "Simulation of arbitrary transmission line networks with nonlinear terminations," IEEE Trans. on Circuits Systems, vol. 38, pp. 418-422, April 1991.

[19] S. Lin, E. S. Kuh, "Transient simulation of lossy interconnects based on the recursive convolution formulation," IEEE Trans. on Circuits and Systems I: Fundamental Theory and Applications, vol. 39, no.11, pp. 879 - 892, Nov. 1992.

[20] W. T. Beyene, and J. E. Schutt-Aine, "Accurate frequency-domain modelling and simulation of high-speed packaging interconnects", IEEE Trans. on Microwave Theory and Techniques, pp. 1941-1947, Oct. 1997.

[21] J. Kolstad, C. Blevins, J. Dunn, and A. Weisshaar, "A new modeling methodology for passive components based on black-box augmentation combined with equivalent circuit perturbation", Proceedings IEEE 13th Topical Meeting on EPEP, pp. 259- 
262, Portland, OR, Oct. 2004.

[22] J. Kolstad, C. Blevins, J. M. Dunn, and A. Weisshaar, "A new circuit augmentation method for modeling of interconnects and passive components", IEEE Trans. Advanced Packaging, vol. 29, no. 1, pp. 67-77, Feb.2006.

[23] M. Elzinga, K. L. Virga, L. Zhao and J. L. Prince, "Pole-residue formulation for transient simulation of high-frequency interconnects using householder LS curvefitting techniques," IEEE Trans. on Advanced Packaging, vol. 25, pp. 142-147, May 2000.

[24] R. Achar, P. Gunupudi, M. Nakhla and E. Chiprout, "Passive interconnect reduction algorithm for distributed/measured networks", TCAS -II:Analog \& Digital Signal Processing, Vol.47, pp. 287-301, April 2000.

[25] D. Saraswat, R. Achar and M. Nakhla, "Restoration of passivity in S-parameter data of microwave measurements", IEEE International Microwave Symposium Digest, June 2005.

[26] B. Gustavsen and A. Semlyen, "Rational approximation of frequency domain responses by vector fitting”, IEEE Trans. on Power Delivery, pp. 1052-1061, July 1999.

[27] D. Saraswat, R. Achar and M. Nakhla, "A fast algorithm and practical considerations for passive macromodeling of measured/simulated data," IEEE 11th Topical Meeting on Electrical Performance of Electronic Packaging, Monterey, CA, pp. 297-300, October 2002.

[28] D. Saraswat, R. Achar and M. Nakhla, "A fast algorithm and practical considerations for passive macromodeling of measured/simulated data", IEEE Trans. on Components Packaging and Manufacturing Technology, Volume: 27, Issue: 1, pp. 57 - 70, Feb. 2004.

[29] W. T. Beyene and J. E. Schutt-Aine, "Efficient transient simulation of high-speed interconnects characterized by sampled data", IEEE Trans. on Components, Packaging, and Manufacturing Technology, Part B, no. 1, pp. 105-114, Feb. 1998.

[30] W. T. Beyene and J. E. Schutt-Aine, "Interconnect simulation using order reduction 
and scattering parameters", IEEE Electronic Components and Tech. Conf., pp. 627$631,1998$.

[31] M. Elzinga, K. L. Virga and J. L. Prince, "Improved global rational approximation macromodeling algorithm for networks characterized by frequency-sampled data", IEEE Trans. on Microwave Theory and Techniques, vol. 48, no. 9, pp. 1461-1468, Sept. 2000.

[32] P. Delsarte, Y. Genin, and Y. Kamp, "On the role of the Nevanlinna-Pick problem in circuits and system theory," Circuit Theory and Applications, vol. 9, pp. 177-187, 1981.

[33] C. P. Coelho, J. R. Phillips, and L. M. Silveira, "Passive constrained rational approximation algorithm using nevanlinna-pick interpolation", Proceedings of Design, Automation and Test in Europe Conference and Exhibition, pp. 923-930, March 2002.

[34] C. P. Coelho, J. R. Phillips, and L. M. Silveira, "A convex programming approach to positive real rational approximation", Proc. IEEE/ACM International Conference on Computer Aided Design, pp. 245-251, November 2001.

[35] C. P. Coelho, J. R. Phillips, and L. M. Silveira, "A convex programming approach for generating guaranteed passive approximations to tabulated frequency-data", IEEE Trans. Computer-Aided Design of Integrated Circuits and Systems, vol. 23, issue 2, pp. 293 - 301, Feb. 2004.

[36] H. Chen and J. Fang, "Enforcing bounded realness of ' $S$ ' parameter through trace parameterization," IEEE $12^{\text {th }}$ Topical Meeting on EPEP, Princeton, New Jersey, pp. 291-294, October 2003.

[37] C. P. Coelho, J. R. Phillips, and L. M. Silveira, "Robust rational function approximation algorithm for model generation", In Proc. 36th Design Automation conf., New Orleans, pp. 207-212, June 99.

[38] J. Morsey and A. C. Cangellaris, "Passive realization of interconnect models from measured data", IEEE $10^{\text {th }}$ Topical Meeting on EPEP, Cambridge, MA, pp. 47 50 , October 2001.

[39] D. Saraswat, R. Achar and M. Nakhla, "Passive macromodels of microwave subnet- 
works characterized by measured/simulated data", International Microwave Symposium Digest, Philadelphia, PA, pp. 999-1002, June 2003.

[40] D. Saraswat, R. Achar and M. Nakhla, "On passivity check and compensation of macromodels from tabulated data", Proc.7th IEEE Workshop on Signal Propagation on Interconnects, Siena, Italy, pp. 25-28, May 2003.

[41] B. Gustavsen and A. Semlyen, "Enforcing passivity for admittance matrices approximated by rational functions", IEEE Trans. on Power Systems, pp. 97-104, February 2001 .

[42] R. Neumayer, F. Haslinger, A. Stelzer and R. Weigel, "On the synthesis of equivalent circuit models for multiports characterized by frequency-dependent parameters", IEEE Trans. on Microwave Theory and Techniques, vol. 50, Issue: 12, pp. 2789-2796, Dec. 2002.

[43] S. Grivet-Talocia, "Passivity enforcement via perturbation of hamiltonian matrices", IEEE Trans. Circuits and Systems I: Fundamental Theory and Applications, pp. 1755-1769, vol. 51, no. 9, Sept. 2004.

[44] L. M. Silveria, I. M. Elfadel, J. K. White, M. Chilukuri and K. S. Kundert, "An efficient approach to transmission line simulation using measured or tabulated S-parameter data," in Proc. ACM/IEEE Design Automation conf., pp. 634-639, June 1994.

[45] W. T. Beyene and J. E. Schutt-Aine, "Interconnect simulation using order reduction and scattering parameters," IEEE Electronic Components and Technology Conference, pp. 627-631, 1998.

[46] S. Min and M. Swaminathan, "Efficient construction of two port passive macromodels for resonant networks", IEEE $10^{\text {th }}$ Topical Meeting on EPEP, Cambridge, Massachusetts, pp. 230-232, October 2001.

[47] D. Saraswat, R. Achar and M. Nakhla, "Circuit compatible macromodeling of highspeed VLSI modules characterized by scattering parameters", Proc. IEEE VLSI Design, pp. 5D-1.1-1.5, Jan. 2006, Hyderabad, India.

[48] Timo Palenius and Jane Roos, "Comparison of reduced-order interconnect macromodels for time-domain simulation", IEEE Trans. on Microwave Theory and Tech- 
niques, vol. 52, Issue: 0, pp. 2240-2250, Sept. 2004.

[49] D. Saraswat, R. Achar and M. Nakhla, "Passive macromodeling of subnetworks characterized by measured data", IEEE International Symposium on Circuits and Systems, pp. 25 - 28, Bangkok, Thailand, May 2003.

[50] D. Saraswat, R. Achar and M. Nakhla, "On passivity enforcement for macromodels of S-parameter based tabulated subnetworks", Proc. IEEE International Symposium on Circuits and Systems, Kobe, Japan, May 2005.

[51] D. Saraswat, R. Achar and M. Nakhla, "Fast passive macromodeling of S-parameter based interconnect subnetworks", IEEE ANTEM, Ottawa, pp. 357 - 360, July 2004.

[52] D. Saraswat, R. Achar and M. Nakhla, "Circuit simulation of S-parameter based interconnects via passive macromodels", IEEE NEWCAS, Montreal, Quebec, pp. 309 - 312, June 2004.

[53] D. Saraswat, R. Achar and M. Nakhla, "Fast passivity verification and enforcement via reciprocal systems for interconnects with large order macromodels", Accepted for second review in IEEE Trans. on VLSI, Nov. 2005.

[54] S. Grivet-Talocia, "On the generation of large passive macromodels for complex interconnect structures", IEEE Trans. on Advanced Packaging, vol. 29, no. 1, pp. 3954, Feb. 2006.

[55] S. Grivet-Talocia, "Improving the efficiency of passivity compensation schemes via adaptive sampling", IEEE 14th Topical meeting on Electrical Performance of Electronic Packaging (EPEP), Austin, TX (USA), pp. 231--234, Oct. 24-26, 2005.

[56] D. Saraswat, R. Achar and M. Nakhla, "Global passivity enforcement algorithm for macromodels of interconnect subnetworks characterized by tabulated data", IEEE Trans. on VLSI, vol. 13, No. 7, pp. 819 - 832, July 2005.

[57] D. Saraswat, R. Achar and M. Nakhla, "Enforcing Passivity for Rational Function Based Macromodels of Tabulated Data”, IEEE 12th Topical Meeting on EPEP, Princeton, NJ, pp. 295-298, October 2003.

[58] S. D. Corey and A. T. Yang, "Interconnect characterization using time-domain reflectometry", IEEE Trans. on Microwave Theory and Techniques, Issue: 9, pp. 2151- 
2156, Sept. 1995.

[59] HSPICE(c) Signal Integrity Guide, V-2004.03, Synopsys, March 2004.

[60] Virtuoso(c) SpectreRF Simulation Option User Guide, Product Version 5.1.41, Cadence Design Systems, November 2004.

[61] Analog Library Reference Guide, Product Version 5.1.41, Cadence Design Systems, December 2004.

[62] S-Parameter Simulation, Agilent Technologies, September, 2004.

[63] V. Mehrmann and D. Watkins, "Structure-Preserving Methods for Computing Eigenpairs of Large Sparse Skew-Hamiltonian/Hamiltonian Pencils", SIAM J. Sci. Comput., 22:1905-1925, 2001.

[64] D. Saraswat, R. Achar and M. Nakhla, "Passive Reduction Algorithm for RLC Interconnect Circuits with Embedded State-Space Systems (PRESS)", IEEE Trans. on Microwave Theory and Techniques, vol. 52, No. 9, pp. 2215 - 2226, Sept. 2004.

[65] D. Saraswat, R. Achar and M. Nakhla, "A methodology for generating compact passive macromodels for high-frequency interconnect and microwave subnetworks," IEEE International Microwave Symposium Digest, pp. 707 - 710, June 2004.

[66] D. Saraswat, R. Achar and M. Nakhla, "A fast algorithm for near optimal passive reduction of high-speed interconnect networks," IEEE Workshop on Signal Propagation on Interconnects, pp. 91 - 94, Heidelberg, Germany, May 2004.

[67] M. E. Valkenburg, Introduction to Modern Network Synthesis. NY: John Wiley \& Sons Inc., 1960.

[68] Y. Saad, Iterative Methods for Sparse Linear Systems. Boston: PWD Publishing Company, 1996.

[69] B. D. O. Anderson and S. Vongpanitlerd, Network Analysis and Synthesis. Englewood cliffs: Prentice-Hall Inc., 1973.

[70] S. Boyd, L. El Ghaoui, E. Feron and V. Balakrishnan, Linear Matrix Inequalities in System and Control Theory. SIAM Studies in Applied Mathematics, vol. 15, 1994.

[71] B. Moore, "Principal component analysis in linear systems: Controllability, observ- 
ability and model reduction", IEEE Trans. Automat. Contr., pp. 17-32, Feb. 1981.

[72] X. Chen and J. T. Wen, "Positive Realness Preserving Model Reduction with Hinf Norm Error Bound," IEEE Trans. on Circuits and Systems - I: Fundamental Theory and Applications, vol. 42, no. 1, pp. 23-29, Jan. 1995.

[73] J. R. Phillips, L. Daniel and L. M. Silveira, "Guaranteed passive balancing transformations for model order reduction," IEEE Trans. Computer-Aided Design, vol. 22, no. 8, pp. 1027-1041, Aug. 2003.

[74] P. Rabiei and M. Pedram, "Model order reduction of large circuits using balanced truncation," Proc. Asia and South Pacific Design Automation Conf., Hong Kong, Hong Kong, Jan. 1999, pp. 237-240.

[75] J. Vlach and K. Singhal, Computer methods for circuit analysis and design. NewYork: Van Norstrand Reinolds, 1983.

[76] W. F. Arnold and A. J. Laub, "Generalized eigenproblem algorithms and software for Algebraic Riccati Equations", Proceedings of The IEEE, pp.1746-1754, Dec. 1984.

[77] P. Benner, V. Mehrmann and H. Xu, "A new method for computing the stable invariant subspace of a real Hamiltonian matrix", Journal of Comput. and Applied Mathematics, vol. 86, no. 1, pp. 17-43, Nov. 1997.

[78] R. Achar and M. Nakhla, Minimum Realization of Reduced-Order Models of HighSpeed Interconnect Macromodels, Chapter III: Signal Propagation on Interconnects, pp. 23-45, Kluwer Publishers, 1998.

[79] T. Kailath, Linear Systems. Toronto: Prentice-Hall Inc., 1980.

[80] C. T. Chen, Linear System Theory and Design. New York: Holt, Rinehart and Win.

[81] K. Zhou and J. C. Doyle, Essentials of Robust Control. Upper Saddle River, N.J.: Prentice Hall, 1998.

[82] G. W. Stewart and Ji-guang Sun, Matrix Perturbation Theory. Boston: Academic Press, 1990.

[83] R. H. Bartels and G. W. Stewart, "Algorithm 432: Solution of the matrix equation $\boldsymbol{A} \boldsymbol{X}+\boldsymbol{X} \boldsymbol{B}=\boldsymbol{C} "$, Commun. $A C M$, vol. 15, pp. 820-826, 1972. 
[84] M. Green and David J.N. Limebeer, Linear Robust Control. Englewood Cliffs, N.J.: Prentice Hall, 1995.

[85] E. Kuh and R. Rohrer, Theory of Active Linear Networks. San Francisco: Holdenday, 1967.

[86] L. Weinberg, Network Analysis and Synthesis, New York: McGraw-Hill Book Company Inc., 1962.

[87] P. A. Parrilo, Structured Semidefinite Programs and Semialgebraic Geometry Methods in Robustness and Optimization, PhD. thesis, California Institute of Technology, Pasadena, California, 2000.

[88] A. J. Laub, “A Schur method for solving Algebraic Riccati Equations”, IEEE Trans. on Automatic Control, vol. 24, no. 6, pp. 913-921, Dec. 1979.

[89] S. Bittanti, A. J. Laub and J. C. Willems, The Riccati Equation. Springer-Verlag Berlin, Heidelberg, 1991.

[90] B. Kagstorm and P. Van Dooren, "A generalized state-space approach for the additive decomposition of a transfer matrix", J. Num. Linear Algbra with Applications, vol. 1, no. 2, pp. 165-181, 1992.

[91] N. Sadegh, J. D. Finney and B. S. Heck, “An explicit method for computing the positive-real lemma matrices," Proceedings 33rd IEEE Conference on Decision and Control, pp. 1464-1469, Dec. 1994.

[92] L. Wei and F. Yeh, "On dual algebraic riccati equations", IEEE Trans. Automat. Contr., vol. 36, no. 4, pp. 511-512, April 1991.

[93] A. Lu and E. L. Wachspress, "Solution of Lyapunov equations by alternating direction implicit iteration", Comput. Math. Appl., vol. 21, no. 9, pp. 43-58, 1991. 